\section{NISTIR 5398}

(supersedes NISTIR 4349,4953, and 5238)

\title{
User's Guide for the PHIGS Validation Tests (Version 2.1)
}

\section{Kevin Brady \\ John Cugini \\ Qiming Wang}

U.S. DEPARTMENT OF COMMERCE Technology Administration National Institute of Standards and Technology

Computer Systems Laboratory Gaithersburg, MD 20899

April 1994 



\section{NISTIR 5398}

(supersedes NISTIR 4349,4953, and 5238)

\section{User's Guide for the PHIGS Validation Tests (Version 2.1)}

\section{Kevin Brady \\ John Cugini \\ Qiming Wang}

U.S. DEPARTMENT OF COMMERCE Technology Administration

National Institute of Standards and Technology

Computer Systems Laboratory Gaithersburg, MD 20899

April 1994

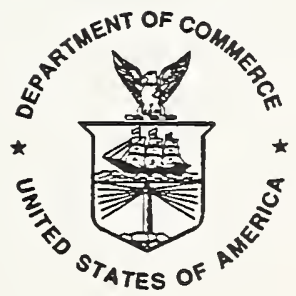

U.S. DEPARTMENT OF COMMERCE Ronald H. Brown, Secretary

TECHNOLOGY ADMINISTRATION

Mary L Good, Under Secretary for Technology

NATIONAL INSTITUTE OF STANDARDS

AND TECHNOLOGY

Arati Prabhakar, Director 

INTRODUCTION • . • • . . . . . . . . . . . . . 1

STRUCTURE AND FORMAT OF PVT SUITE . . . . . . . . . 2

2.2

Tree Structure • • • • • • . • . . . . . . 2

2.3

Modules

2.4

Subroutine Libraries . . . . . . . . . . . . 3

2.5

$2 \cdot 5 \cdot 1$

$2 \cdot 5.2$

$2 \cdot 5 \cdot 2.1$

2.5 .2 .2

Order

$2 \cdot 5 \cdot 2.3$

$2 \cdot 5.2 .4$

Module Documentation

$2 \cdot 5 \cdot 2 \cdot 5$

2.5 .2 .6

$2 \cdot 5 \cdot 3$

2.5 .4

$2 \cdot 5.5$

2.5 .6

$2 \cdot 5 \cdot 6.1$

$2 \cdot 5 \cdot 6.2$

2.6

2.6 .1

2.6. 2

2.6.2.1

2.6 .2 .2

2.6 .2 .3

2.6 .3

2.6 .4

2.6 .5

2.6 .6

2.6 .7

2.6 .8

2.6 .8 .1

2.6 .8 .2

2.6 .8 .3

2.6 .8 .4

2.6 .8 .5

$2.6 .8 \cdot 6$

2.6 .8 .7

2.7

2.7 .1

2.7 .2

Introductory Headings . . . . . . . . . . 4

Semantic Requirements . . . . . . . . . 5

Related Functions . . . . . . . . . . 6

Related Data Structures . . . . . . . 6

Basis In The Standard . . . . . . . 7

Test Cases... . . . . . . . . . . 7

Module Cross-references . . . . . . . 7

Comments . . . . . . . . . . . 8

Local Dictionary . . . . . . . . . . . . 8

Semantic Cross-references . . . . . . . . 8

Local Subroutines . . . . . . . . . . . 8

Program Design . . . . . . . . . . . . . 8

Headings . . . . . . . . . . . . . 8

Logic And TCs . . . . . . . . . . . . . 9

Source Code . . . . . . . . . . . . . 10

Language . . . . . . . . . . . . . . . 10

Generation of C Version of PVT From Fortran . 10

Use of F2C As Translator . . . . . . . . 11

Customization of F2C Libraries . . . . . . . 11

Interface Between Fortran And C Bindings . . 11

All Variables Declared . . . . . . . . . 12

Standard PHIGS Names . . . . . . . . . . 13

Dummy Parameters . . . . . . . . . . . . 13

Program Banner . . . . . . . . . . . . . 13

Common • . • • • • • • . . . • • • • • . 13

Special Characteristics of C Code . . . . . 13

Prototyping . . . . . . . . . . . . . . 14

File Handling . . . . . . . . . . . . 14

Pack/Unpack . . . . . . . . . . . . . 14

Character Strings . . . . . . . . . 15

Error Handling . . . . . . . . . . . 16

Parameter Passing . . . . . . . . . . 17

Array Indexing . . . . . . . . . . . 17

Ubiquitous Subroutines . . . . . . . . . . 18

Initialization And Clean-up of Test Programs . 18

TC Subroutines . . . . . . . . . . . 18

Message Subroutines . . . . . . . . . 19

2.7 .4

CHKINQ 
3.1

3.1 .1

3.1 .2

3.2

3.2 .1

3.2 .2

3.2 .3

3.2 .4

3.2 .5

3.2 .6

3.2 .7

3.2 .8

3.2 .9

3. 2.10

3.2 .11

3.3

3.4

4

4.1

4.2

4.2 .1

4.2 .2

4.2 .3

4.2 .4

4.2 .5

4.2 .6

4.2 .7

4.2 .8

4.2 .9

4.2 .10

4.3

4.4

4.4 .1

4.4 .2

4.4 .3

4.4 .4

4.4 .5

4.4 .5 .1

4.4 .5 .2

4.4 .6

4.4 .7

4.4 .8

4.5

4.6

INSTALLATION . . . . . . . . . . . . . 20

File Storage .............. 20

Hierarchical Format . . . . . . . . . 20

Alternative Storage Strategy For Subroutines . 21

Customization of Code . . . . . . . . . 22

Naming PVT Configuration File . . . . . . . 22

Special Processing For Opening PHIGS . . . . 22

Random Number Generator . . . . . . . . . 23

Naming Individual Message File... . . . . 23

Resolution of Parameters For <Open Workstation> 23

Providing Valid Names For Archive Files . . . 23

Time-stamping Message Files ......... 24

Operator Communication . . . . . . . . . 24

Appending To Global Message File . . . . . 24

Control of C Prototyping............ 24

Special Characters . . . . . . . . . . 25

Compile Subroutines . . . . . . . . . . 25

Procedures For Batch Processing . . . . . 26

OPERATION ................... 26

PVT Sessions And Workstations . . . . . . 27

Running INITPH For PVT Configuration..... . 28

Parameters For Opening PHIGS . . . . . . . 29

Parameters For Opening Workstations . . . . 30

Control of Messages ............ 30

Control of Error File . . . . . . . . . 31

Control of Randomization . . . . . . . . . 31

Primary Workstation Support For Graphical

Output . . . . . . . . . . . . . 31

Control For Prompting The operator. . . . . 32

Control For Operator Responses To Prompt . . . 32

Location And Size of The Dialogue And Echo

Areas . . . . . . . . . . . . 32

Ratio of Meters To DC Units For The Primary

Workstation . . . . . . . . . . 32

Background Documentation . . . . . . . . 33

Execution And Interpretation . . . . . . . . . 33

Testing Error Handling . . . . . . . . . . 33

Language Specific Tests . . . . . . . . 33

Failure To Compile or Link . . . . . . . 34

Failure To Complete Execution . . . . . . 34

Operator Interaction ........... 35

Format For Answering Questions . . . . . 35

Special Processing of operator Responses . . 35

Completion of Execution And Message Type . . 36

Analysis of Results . . . . . . . . . 37

Troubleshooting . . . . . . . . . . . 37

Session Completion . . . . . . . . . . 38

Customization For Debugging . . . . . . . 38 
REFERENCES

APPENDIX A

GLOBAI VARIABLES

APPENDIX B

PVT TREE STRUCTURE

APPENDIX C

VERSION HISTORY OF THE PVT

APPENDIX D

TEST PROGRAM CHARACTERISTICS

APPENDIX E

DICTIONARY OF SUBROUTINES AND FUNCTIONS

APPENDIX F

EXAMPIES OF COMMAND PROCEDURES

APPENDIX G

BUILDING THE PVT IN UNIX

APPENDIX H

SOFTWARE METRICS

APPENDIX I

FUNCTION CROSS-REFERENCE

APPENDIX J

DATA STRUCTURE CROSS-REFERENCE

APPENDIX K

STANDARD SPECIFICATIONS CROSS-REFERENCE

APPENDIX L

MODULE CROSS-REFERENCE 

(Version 2.1)

by

Kevin Brady

John Cugini

Qiming wang

\begin{abstract}
The PHIGS Validation Tests (PVT), developed by NIST, consist of a large set of Fortran and C programs which may be used to test how well implementations of PHIGS conform to the standard. The tests are organized into a hierarchical structure of modules which corresponds to the conceptual overview of the standard. The tests are associated with the standard via a set of semantic requirements which are derived directly from the standard. Cross-reference tables allow the user to find tests relating to specific PHIGS functions and data structures. Directions for installation and operation of the tests are included.
\end{abstract}

KEYWORDS: conformance testing; graphics standards; PHIGS; testing of software; validation of software 
PHIGS stands for Programmer's Hierarchical Interactive Graphics system. The PHIGS standard defines a set of functions to be used by a programmer to manipulate and display 3-D graphical objects. For a full description of the features of PHIGS, see [PHIGS89]. The standard has been approved by the American National Standards Institute (ANSI) as ANSI X3.144-1988, by the International Organization for Standards (ISO) as ISO 9592-1:1989, and by the Federal government as Eederal Information Processing Standard (FIPS) 153.

The PHIGS Validation Test (PVT) suite is a product of the Computer systems Laboratory (CSL) of the National Institute of Standards and Technology (NIST). The function of this suite is to test whether implementations of PHIGS conform to the PHIGS standard. The PVT is available to individuals and organizations for use in developing or testing PHIGS implementations. CSI uses version 2.1 of this suite to validate PHIGS implementations which have been submitted for testing.

There are some aspects of the PHIGS standard which can reasonably be construed in various ways. The PVT suite embodies CSI's best technical judgment concerning the standard's requirements for conforming implementations. CSI intends to update the PVT suite periodically to reflect official ANSI or ISO interpretations that conflict with the assumptions upon which the current version of the PVT is based.

Version 2.1 includes tests for error handling and updates the PVT to reflect official interpretations of the PHIGS standard. See Appendix $C$ for a description of the coverage provided by the various versions of the PVT.

This document describes the general rules and procedures for using the PVT suite. Detailed information for specific tests may be found in the module documentation, as described in section 2.5 . For a general discussion of PVT design issues, see [CUGI90] and [CUGI91].

We welcome any comments or suggestions regarding the PVT. Such comments may include reports of errors in the PVT, suggestions for additional test cases, interpretation questions, or any other ideas on how to improve the PVT. Please send all correspondence, including questions about PHIGS validation and obtaining the PVT, to:

Project Leader, PHIGS Validation Tests

Computer systems Laboratory

National Institute of Standards and Technology

Bldg. 225, Room A-266

Gaithersburg, MD 20899 
This report identifies certain commercial software products in order to illustrate some of the concepts discussed herein. Such identification does not imply recommendation or endorsement by NIST.

\section{STRUCTURE AND FORMAT OF PVT SUITE}

The PVT suite is implemented as a set of documentation files and source code files. This section describes the relationship among these files, and the format conventions they follow.

\subsection{Tree structure}

The PVT suite is organized in a hierarchical, or tree, structure. This tree closely resembles the organization of section 4 of the generic ISO PHIGS standard, which contains the basic conceptual description of PHIGS. See Appendix B for the complete description of the PVT tree.

Concretely, the tree structure is expressed by storing the modules of the PVT system in a hierarchical file directory, such as that supported by VAX/VMS, Unix, and MS-DOS. For illustrative purposes we shall adopt a neutral naming convention, wherein the levels of a file structure are separated by the " " character, for example: PVT 05 03 DOC.TXT as the name of the documentation file for module 05 03. As distributed, the root of the PVT tree contains a number of system files:

a. user-guide.prt: user's guide for the PVT; the file you are now reading

b. sys-dict.prt: dictionary file to specify the identifying numbers of PHIGS functions and data structures

c. pgm-char.prt: contains a list of all programs, explicitly numbered, together with their characteristics

d. initph.f or initph.c: source code for initializer of PVT configuration file

e. oprcmt.f or oprcmt.c: source code for program to allow entry of operator comments outside the usual context of a test program (see section 4.4 .3 )

f. complt.f or complt.c: source code for program to test completion of validation session (see section 4.5)

g. sublib.f or sublib.c: source code for global subroutine library 
h. trans-sublib.f or trans-sublib.c: source code for transformation subroutine library

i. errlib.f or errlib.c: source code for error handler library

\subsection{Modules}

There is at most one PVT module per node in the tree. Every leaf node contains a module. A non-leaf node may contain a module, or may be empty, serving simply to organize the system. Every module contains exactly one documentation file, conventionally named DOC.TXT, and one or more program files, named p01.f, p02.f, etc. for Fortran, or p01.c, etc in the case of $C$. The documentation file contains the set of semantic requirements (SRs) for the module, together with the design for each of the module's programs. The programs contain the test cases (TCs) for the module. Format details are explained below. See Figure 1 for a schematic diagram of the structure of a module.

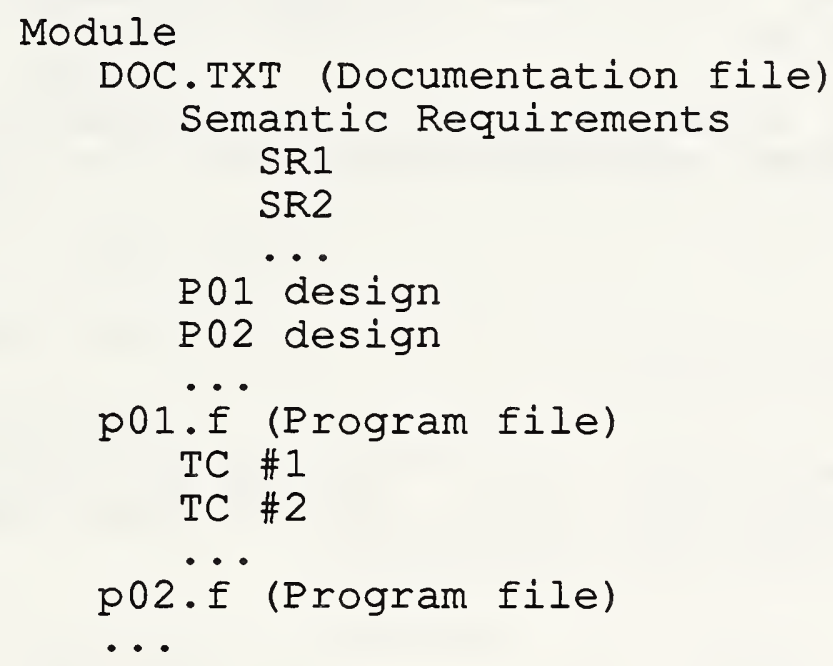

Figure 1: File Structure of a Module

\subsection{Subroutine Libraries}

The only other entities of the PVT system not in the root, besides the documentation and source code files, are the subroutine libraries (also in source code) used by the main programs. These libraries are in the tree structure, and are associated not with individual modules, but with the set of modules below them in the 
tree or at the same level. Thus, if programs in modules 05 01 01, 05 01 03, 05 02, and 05 04 all use a library of common subroutines, this library will reside in PVT 05. Libraries are named sublib.f. There is a global subroutine library, at the PVT level, containing routines used throughout the PVT system.

Finally, there are two special-purpose libraries, anomalously named trans-sublib.f and errlib.f in the root node. The former contains routines that simulate the geometric transformation utility functions of PHIGS and perform other mathematical functions. The latter contains the PVT-defined version of PERHND (the Fortran error handler) or perr_hand (the C error handler).

The subroutines are self-documented; each starts with a brief description of its purpose and of the meaning of its parameters. See Appendix E for a dictionary of the names and locations of all PVT subroutines.

\subsection{Order}

There is never any order-dependence between modules, and usually there is no required order for program execution within a module. Following the PVT tree structure, depth-first, may be the most natural way to proceed through the modules, but there is no requirement to do so.

Module 11.02 is an exception to this rule: it checks the PHIGS header files for the $\mathrm{C}$ binding and its programs must be run in sequence.

\subsection{Module Documentation}

Module documentation is written for a reasonably knowledgeable PHIGS user. Questions about PHIGS itself must be answered by reference to the standard [PHIGS89].

Figure 2 contains a schematic outline of the module documentation. Refer to it, or to an actual example of module documentation when reading this section.

\subsubsection{Introductory Headings -}

The first heading, "TITLE", contains a brief phrase describing the main topic of the module. The second heading, "MODULE\#" contains the identifying number of the module, which may be simply related to its location in the file hierarchy, e.g. the files of MODULE\# 02.01 .02 are in the PVT 02 01 02 sub-directory. All level numbers are two-digit. The third heading, "DESCRIPTION" contains a free-format paragraph explaining the scope of the module. 


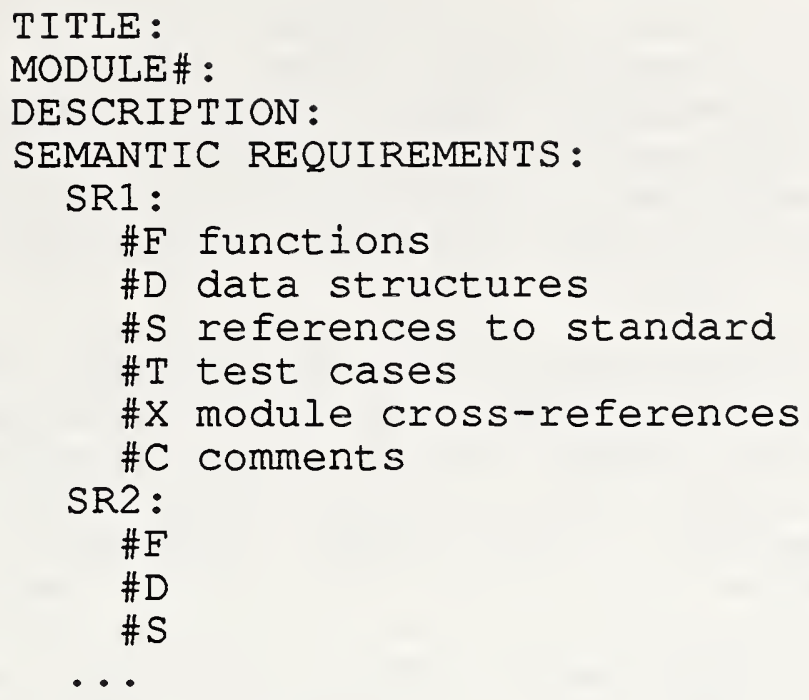

Figure 2: Format of Module Documentation

\subsubsection{Semantic Requirements -}

Next comes the list of semantic requirements associated with the PHIGS feature under test. These are assigned sequential identifying numbers, which will be referred to by the relevant TCs. 
Each SR is a simple declarative sentence, stating some requirement on the behavior of conforming implementations. Although intended to be clear and unambiguous, these have to be read in a "reasonable" way, with appropriate assumptions about context. The SRs are not "conditionalized" into absolute truths by explicitly stating all the normal pre-conditions that may apply (e.g. if a structure is open and if the system has space left to allocate, and if there is no power failure, then ...).

The SRs use the terminology of the standard when referring to functions or data structures. By convention, angle brackets are used when referring to the generic names of functions, e.g. <set element pointer>. Note that the words "valid" and "realizable" have a technical meaning: the former indicates simply that a parameter is accepted by a function without generating an error, while the latter indicates that the implementation must actually be able to render the graphical attribute in question. The wording of the SR usually applies to the generic standard, and is not language-dependent. Of course the SRs for the language specific modules are themselves language-dependent.

Immediately following each SR there are a number of associated fields, one per line, each prefaced by a "\#". The first four of these, \#F, \#D, \#S, and \#T are mandatory.

\subsubsection{Related Functions -}

\#F is used to list the functions associated with this SR, i.e. those whose behavior is at least partially constrained by the SR. Functions are referred to by an identifying number (1-324) which corresponds to the order in which they appear in section 5 of the standard. The sys-dict.prt file contains a complete table of function numbers.

\subsubsection{Related Data Structures -}

The \#D entry lists the data structures which are relevant to this SR, i.e. those whose contents are changed or inspected in the course of checking the SR. The data structures are referred to by an identifying hierarchical number which reflects the organization of section 6 of the standard. The sys-dict.prt file contains a complete table of data structure numbers. Note that the data structure number need not be a leaf node. If the SR affects everything under a non-leaf node, then an entry for that node implicitly covers all those below it. 
2.5.2.3 Basis In The Standard -

The \#S entry lists those parts of the standard upon which the $S R$ is logicaliy based. These references are in the form: <section-number> / <page-number> / <paragraph-number>, all of which refer to the 1989 ANSI/ISO PHIGS standard, [PHIGS89]. The rules for counting paragraphs within a page of the standard are as follows. Whatever partial chunk of text is at the top of the page is number 1. Subsequent paragraphs are deemed to start by a blank line (even if half-height) followed by text at extreme left hand margin (not indented), but not counting section titles. Thus, page 38 has 7 paragraphs: 1 partial completing section 4.5 .1 , and 2-7 in section 4.5.2. Figures are associated with the preceding paragraph. For example, page 46 has 3 paragraphs with figure 7 as part of the first.

When an SR is based on one of the PHIGS language bindings, its \#S entry is in the form <language> / <section-number> / <page-number> / <paragraph-number>, and refers to either [PHFOR90] or [PHC91].

The page numbers of the relevant functions, as listed under $\# F$, are not included; if a function is relevant, it is to be assumed that its description in section 5 of the standard will have some bearing on the SR. If there is no basis in the standard for the SR besides the description of the relevant functions, an " $n$ " appears in the \#S entry.

\section{5 .2 .4 Test Cases -}

Each SR, under the \#T entry, lists the TCs which depend on it. The format of each TC reference is: $\mathrm{P}$ <program-number> / <test-case-number>. The test case number simply refers to its static sequential position in the text of the program. Note that not all TCs are always executed; under certain conditions specific to the implementation, some may be skipped. Moreover, some TCs occur in loops and are executed several times. Therefore the textual position of TCs in the code may not correspond to the executed order of TCS.

\subsubsection{Module Cross-references -}

In some cases an SR may be significantly related to the topics of several modules. When this happens, we assign the SR to the most strongly related module, and use the \#x entry to list the other relevant modules. 
The \#C entry is for free-form comments to allow explanation of any unusual aspect of the SR. For instance, if the support in the standard for the $S R$ is obscure or indirect, the comment field might be used to explain the validity of the SR.

\subsubsection{Local Dictionary -}

Since the \#F and \#D entries under the sRs are not self-explanatory, the documentation supplies the subset of the global function and data structure dictionary needed to decode the entries of this module.

\subsubsection{Semantic Cross-references -}

If this module is pointed to by an entry in the \#x field of another module, that pointer is noted here in the format <module-number>/SR<sr-number>. For example, if SR4 in module 02.01 contains a \#X entry for module 04.01.01.01, then the latter module will have "02.01/SR4" as a semantic cross-reference entry.

\subsubsection{Iocal Subroutines -}

If the programs of this module use any local subroutines (i.e. any besides those in the root-level global library), it is noted here. For detailed documentation of the logic and parameters of subroutines, please see the source code.

\subsubsection{Program Design -}

The second major part of the documentation of the module is a description of the programs and TCs which actually test the SRs of the module. The programs are numbered sequentially. Except for the language specific modules, the design is intended to be language-independent, relying only on the generic standard and not on language binding details.

\subsubsection{Headings -}

Each program design starts with a "PROGRAM" header, which includes the ordinal number of the program within this module and a descriptive title. The next entry, "CHARACTERISTICS" contains a four-character code (" $y$ " or " $n$ " for yes or no) to indicate various 
properties of the program:

1. requires graphical output features of the primary workstation, which must be of category OUTIN or OUTPUT,

2. requires graphical input features of the primary workstation, which must be of category OUTIN or INPUT,

3. reserved for future use; currently always " $n$ "

4. the need for visual (or other human) interpretation, i.e. the program cannot completely determine pass/fail results, but must rely to some degree on the operator

The CHARACTERISTICS are useful in setting up procedures to run the test suite. Programs with "--nn" are passive tests and may be run without operator intervention. Programs with "nn--" are workstation independent tests and need not be repeated when testing various workstation types (see section 4.1).

The last heading is "OPERATOR SCRIPT." This contains the instructions to be followed by the operator when running the test. If there is no need for operator intervention, then this entry will say "passive test." See section 4.4 .5 for general operator instructions.

Each program design is terminated explicitly by an "END PROGRAM" heading, followed by the identifying ordinal number of the program.

\subsubsection{Logic And TCs -}

The core of each program design is a body of pseudo-code which describes the flow of logic and data representation within the program. It should be clear from this pseudo-code why the embedded TCs are supposed to work. The pseudo-code describes only the logic of the program relevant to the TCs; incidental processing, such as opening PHIGS, or opening a structure is not included. The goal is to give the user an understanding of the basic logic of the code, not to depict all the programing details. For the latter, one can consult the code itself.

The style of the pseudo-code is meant to be informal and self-explanatory. Only a few common control structures are used, such as looping, if-then-else, and goto. Labels (the object of goto statements) begin in column 1 and terminate with a colon. The heading "TEST:" heralds the beginning of a test case. This is followed on the same line by "\#SR" and then a list of the SRs upon which this TC is logically based. Beginning on the next line is the textual statement of the expected (correct) result of the test, surrounded by double-quotes. The text is unique within the program 
and thus serves as the identifier of the TC.

For interactive tests, prompts to the operator are indicated by "OPQA" (for operator question and answer), followed by a slash, a topical heading in upper case, and then the question itself, e.g:

OPQA/EDGE FLAG INDICATOR: Which triangles have visible edges?

The result of each test is recorded by executing either a "pass" or "fail" procedure, denoted in the pseudo-code simply by those words. Every TC should cause execution of either one or the other (but not both, of course) of these. In many cases, where the result depends directly on a single condition, instead of coding:

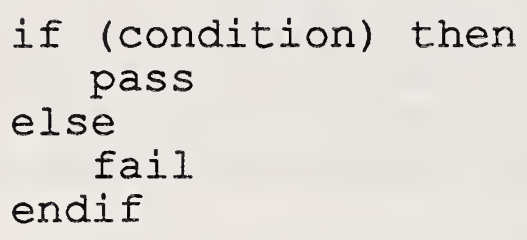

we use the short-hand form:

pass/fail depending on (condition)

\section{6 source code}

In this section we discuss characteristics which pertain to the source code of the PVT system. The code itself is written to be comprehensible by a PHIGS- and Fortran-literate reader. Since the $C$ version of the PVT is generated automatically from the Fortran version, it is somewhat less readable. You may wish to use the corresponding pseudo-code or Fortran code as an aide to understanding the $\mathrm{C}$ code.

\subsubsection{Language -}

The language of version 2.1 of the PVT system is full ANSI Fortran (ANSI X3.9-1978) as defined in [FORT78] or ANSI C (ANSI X3.159-1989) as defined in [C1989]. The language binding to PHIGS is the full Fortran binding [PHFOR90] or C binding [PHC91].

\subsubsection{Generation of C Version of PVT From Fortran -}

The most desirable approach for writing a $C$ binding test suite would have been to start with the design document for each program, and have written new $C$ code to perform the tests outlined. However, due to the limited resources available to the NIST, a 
Fortran to $\mathrm{C}$ translator was used to produce the $\mathrm{C}$ binding test suite.

Using the translator meant starting with well-designed and tested Fortran code, and using that code to generate the $C$ binding code. All of the design and programming effort was expended on the Eortran code are carried over to the $C$ binding test suite through the translator with minimal effort.

\subsubsection{Use of E2C As Translator -}

The translation utility used was the public domain f2c (Fortran to C) converter, developed by AT\&T and Bellcore labs. After evaluating several proprietary and other public domain packages, this converter was chosen for two reasons:

1. the $C$ source code generated from the Fortran code required no further modification, and

2. the purchase of additional software by PVT users to perform a validation is not necessary.

2.6.2.2 Customization of F2C Libraries -

The directory structure of the f2c utility was altered slightly to decrease the number of libraries created. The libraries libF77 and libI77 have been combined into a single library: libf2c. Since all of the code has been translated, the translation utility $f 2 \mathrm{c}$ itself is not contained in the distribution. The directory pvt/F2C contains the source code for the subroutine library (see Figure 3). Since the code has already been translated, only the subroutine library (i.e. libf2c) need be present to link the programs.

The f2c source code itself has not been altered in any way, and sites that have f2c installed as a system library may use their own version.

\subsubsection{Interface Between Fortran And C Bindings -}

Once the Fortran code was translated by the f2c utility, the generated $C$ code still calls the PHIGS Fortran library. To interface this translated code to the PHIGS C library, a "layer" of $C$ code was written between the translated test code and the PHIGS C library (see figure 3). Each PHIGS Fortran routine has an equivalent layer routine where the first letter has been changed to an " $n$ " (e.g. the PHIGS routine ppl becomes the layer routine npl, 


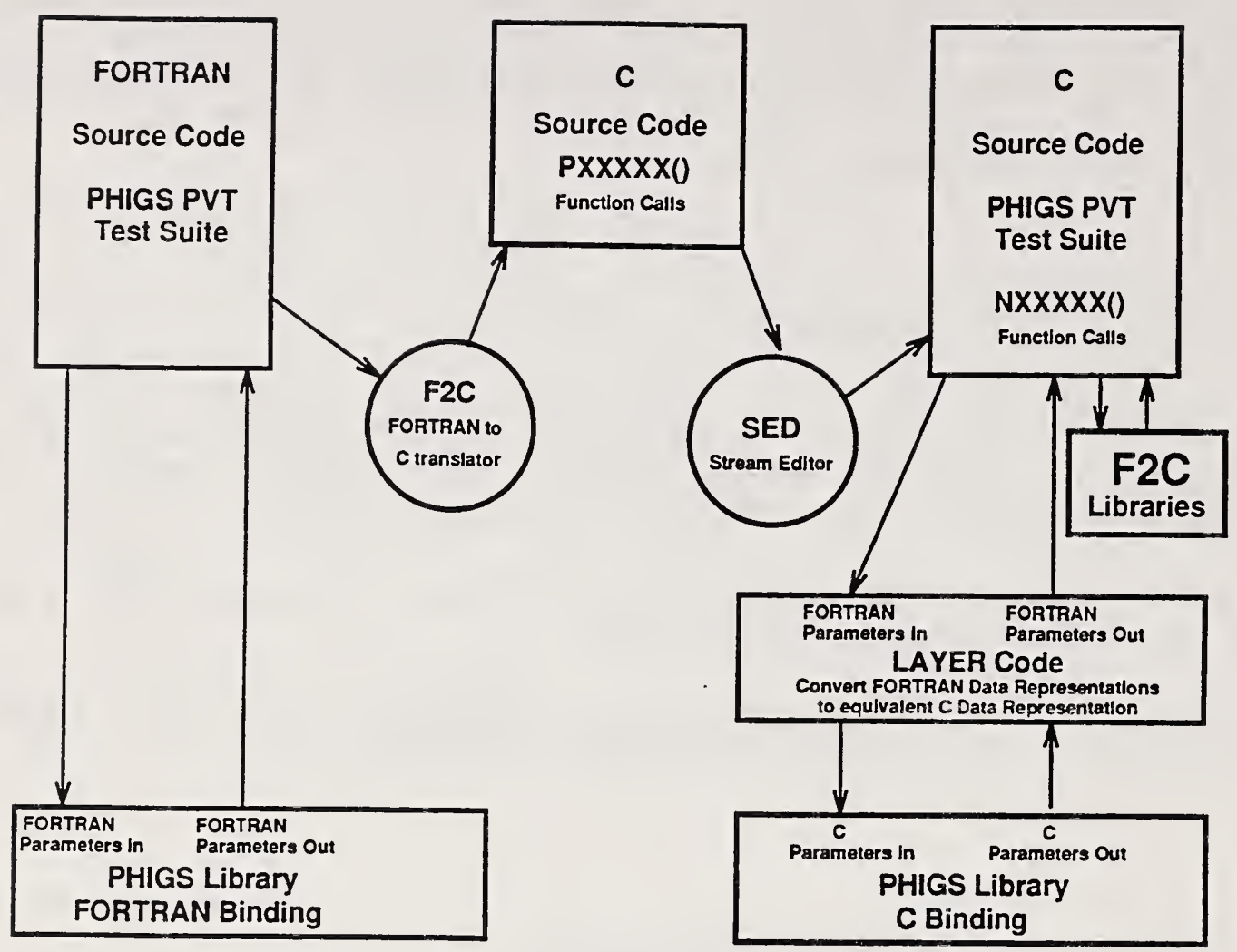

\section{Figure 3: PVT Structure for C binding}

and is contained in the file npl.c within the LAYER sub-directory). Each subroutine in the layer is documented with both the FORTRAN and the $C$ parameters and their meanings. Within the generated $C$ code, references to Fortran/PHIGS routines, which all begin with "P", are automatically altered to begin with "n".

The layer routine accepts the Fortran input parameters and converts them into the equivalent $C$ structures required by the $C$ binding. The equivalent PHIGS C library call is then made (e.g. ppolyline for ppl), allocating space if necessary. The output parameters (if any) from the PHIGS C library call are then extracted and returned in the Fortran output parameters. The routine also frees any space it previously allocated.

This approach was possible since the standard requires the same information to be present for each function, regardless of the binding. Therefore, even though the syntax of the two calls differ, they both process the same information. 


\subsubsection{All Variables Declared -}

Although not required by the Fortran standard, the code explicitly declares the type of all program variables. We believe this is useful for several purposes: it helps avoid certain programming errors (e.g. misspelled variables can be detected by having the compiler flag undeclared variables) and enhances self-documentation of the code.

\subsubsection{Standard PHIGS Names -}

Where appropriate, the Fortran code uses the standard symbolic constant names recommended in section 6 (Enumeration Types) of the Fortran binding standard [PHFOR90]. Since the C code is generated from the Fortran, the standard C macro definitions in section 6 of [PHC91] are not used. For example, the linetype dashed is denoted by PLDASH, not by PLINE_DASH.

\subsubsection{Dummy Parameters -}

When the code invokes a PHIGS function some of whose output parameters are not used in the subsequent logic, these parameters are assigned names in the format: <type>DUM<digit>, where <type> is "I" for integer, "R" for real, " $L$ " for logical, and "C" for character, and where <digit> is some differentiating digit. This tells the reader which parameters are relevant to the logic of the program and which are incidental.

\subsubsection{Program Banner -}

Each test program starts with comment lines forming a banner. This banner identifies the program with a unique "TEST NUMBER" and a "TEST TITLE". These appear in a box of asterisks. The format of the test number is <module-number> / <program-number>. For example, "04.03.01/02" identifies program number 2 in module 04.03 .01 . Thus, the program's file name is p02.f in directory PVT 04 03 01.

\subsubsection{Common -}

Each test program contains a set of identical declarations for certain variables in the common areas GLOBNU and GLOBCH. These are used to convey information within the system. For a full description of each of these global variables, see Appendix A. 
This section describes some of the conventions adopted for the $C$ version of the PVT. A major constraint was the need to be compatible with the code produced by the f2c translator.

\subsubsection{Prototyping -}

Prototyping is a mechanism by which the expected parameter types for an invoked function can be declared and checked against actual usage. Prototyping is allowed by the $C$ language standard and all code generated will, by default, use it [C1989]. However, for those compilers that do not support prototyping, this can be suppressed (see section 3.2.10, below).

\subsubsection{File Handling -}

In PHIGS, a file, as an input parameter, appears in <open phigs>, <open archive file>, <error handler>, <error loggings, and as an output parameter, in <inquire open archive file>. The PHIGS FORTRAN binding assigns the filename to the type integer, and in most instances is associated with a logical unit number. The PHIGS $c$ binding, however, assigns the filename to a character string.

FORTRAN addresses files using the integer unit number with which the file was opened. The C language, on the other hand, addresses a file by the file pointer with which it was opened. These two types differ greatly and there is no one-to-one correspondence between them. The C tests must use a table that is kept by the f2c code which keeps track of FORTRAN unit numbers and the $\mathrm{C}$ files they represent. A number of the layer code routines include the f2c header file "fio.h". These routines deal with file handling and must search the file table to match a FORTRAN unit number to a $C$ filename. An array (of default size 100) is created at the start of each program. Array index [i] contains the information on logical unit i (e.g. array[10] contains the information on logical unit 10). This information should not affect any routine provided in the test suite, for they correctly handle each situation.

\subsubsection{Pack/Unpack -}

The FORTRAN binding has two functions, pack and unpack, which convert data from arrays of integer, real and character data to and from an array of 80 character records. This latter array, in turn, may be used as a parameter to subsequent PHIGS calls. However, the $C$ binding has no such functions (it uses large structures to accomplish this same purpose). Therefore, the layer code for pack 
and unpack does not encode the data into the character arrays. Rather, it defines a general purpose type (see struct.h in pvt/LAYER) to hold such data.

The layer code for pack and unpack (nprec and nurec) will move data into and from the input arrays to a structure defined in struct.h, contained in the LAYER directory. Since NIST is coding pack and unpack (not the implementor), the format for data records is set by us. The current approach is to overlay a structure (special typedef) which holds 20 integers, 20 reals, and 5 strings on top of the raw $80 \times N$ area defined in FORTRAN. This method requires that 592 bytes of storage space be available, resulting in the minimum datrec declaration being $80 \mathrm{x} 8$. Since the test code adheres to this requirement and the method simulates the FORTRAN implementation exactly, the data storage will be totally transparent to the user.

The layer code for functions having input parameters of type data-record must be aware of the datrec format and use it correctly. These functions then face only the familiar problem of re-formatting FORTRAN style data into $C$ style data to be passed to the equivalent $C$ functions. The only difference is that in this case, NIST defined the FORTRAN-style data, instead of the PHIGS standard. This makes sense, since the FORTRAN binding specifically does not define the internal format of data-record - it mandates only that pack stores the data, and that unpack is capable of retrieving the same data.

Using the $C$ language, this new data structure is defined as a parameter to each of the functions that use it, instead of declaring it as the FORTRAN $80 \times \mathrm{N}$ array of characters. Since the FORTRAN main program does not manipulate these arrays in any way (it only passes them to the subroutines), the actual content of the array is never known by the main program. The subroutines, however, receive the array as a pointer to a structure (defined in struct.h). The data from the arrays is stored in the structure and the array passed back. This array is passed by the main program to one of the PHIGS functions that uses it. Each of those functions again declares the received array as type pointer to structure (e.g. a structure pointer), and interprets the structure the same way it was packed, allowing the data to be retrieved and used. There is overhead in this method since EORTRAN requires two steps (pack data, use data), and $C$ requires only one (use data). The C code must therefore emulate the FORTRAN code to limit the amount of changes that must be performed by hand on translated code.

\subsubsection{Character Strings -}

Character strings are represented differently in the FORTRAN language than the $C$ language. In FORTRAN, the length of a character string is declared as a property of the variable itself. In $C$, a string can be of any length and a null termination 
character signals the end of the string. The f2c translator, therefore, represents each FORTRAN character variable as two C data types. The first is the length of the string, and the second is the string itself. Each subroutine call in FORTRAN that contains a character variable is translated into a C function call with an extra parameter for each character variable added on to the end. The lengths of each string are conveyed in these extra variables. The layer code is written to account for these length variables. Therefore, in the LAYER code, some PHIGS functions have more parameters than the standard requires. These parameters are added to hold the lengths of the strings to be received. For example the EORTRAN call to <text>:

$\begin{array}{ll}\text { REAL } & \text { PX, PY } \\ \text { CHARACTER* (*) } & \text { CHARS }\end{array}$

$\cdots$

CALL PTX ( PX, PY, CHARS )

is translated to:

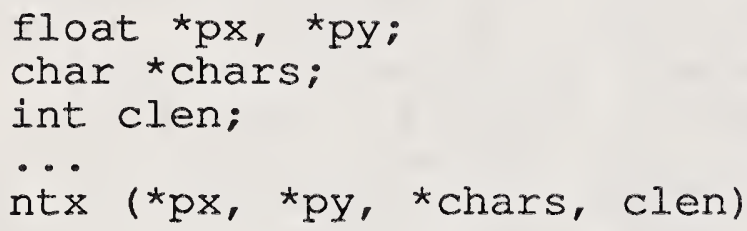

Notice the extra parameter clen. This variable is added to convey the length of the character string chars. Each routine that uses strings follows this convention of the translator. Since the length is known, the layer code checks for variables that are too small to hold data returned from inquires, and will return the PHIGS error 2001 (Ignoring function, output parameter size insufficient) if they are found.

\subsubsection{Error Handling -}

The FORTRAN standard defines the subroutine PERHND as the name of the error handling routine. A user can write his own error handling subroutine, but must call it PERHND. To have this routine invoked, most implementations require the user to link his PERHND routine before the system routines. The $\mathrm{C}$ standard defines the function perr hand to perform the equivalent error handing function.

The Fortran test code makes extensive use of the NIST-defined PERHND routine to determine if a failure is so great that the test code must abort immediately. In order to preserve this functionality, the following steps were taken. 
1. All explicit calls to PERHND are translated to calls to nerhnd (done as usual for other calls to PHIGS functions).

2. In the layer code, nerhnd translates the 3rd parameter (error file) into a form acceptable to perr.hand.

3. After automatic translation of PERHND from Fortran to C, its name is changed to perr hand, and it is altered to accept a character string as the 3 rd parameter, to agree with the PHIGS/C binding.

This procedure allows invocation of the error handler in $C$, either by the implementation's error signalling or by explicit calls to PERHND in the Fortran code and also supports both user-defined and implementor-defined error handling.

Another error handling issue is the PHIGS/C function pset err hand. This function designates an alternate routine (other than the system default perr hand) to be used as the error handler. It has no equivalent in Fortran and is tested in the C-specific module 09.01.11.

All of these changes should be totally transparent to the user and are described here only for the purpose of clarifying the code.

\subsubsection{Parameter Passing -}

Another common problem encountered when changing between languages is the way parameters to functions are passed. FORTRAN parameters are passed by reference or value, depending on whether the parameter is a variable or some other expression. The FORTRAN standard states that constants (e.g. 3), expressions (e.g. X+2) and constant expressions (i.e. variables declared as parameters), declared as parameters to functions, may not be changed in those functions. This would require parameters to be passed by value. However, it is not specified that they cannot be passed by reference. To avoid this problem, the translator passes all variables by reference. To prevent problems with constants and expressions, they are first assigned to a newly created temporary variable, and then the address of that variable is passed to the function. As an example:

CALI SUB $(3, x, X+3) \quad\{$ value, reference, value $\}$

is translated to:

$$
\begin{aligned}
& \text { int c_1, c_2; } \\
& \text { c-1 } 1=3 ; \\
& \text { c-2 }=x+3 ; \\
& \text { sub (\&c_1, \&x, \&c_2); }
\end{aligned}
$$


Arrays and regular variables are already passed by reference, and there is no distinction between input or output variables. The layer code receives all variables as pointers.

\subsubsection{Array Indexing -}

The last problem encountered dealt with array indexing which is the result of another difference between the $C$ and EORTRAN languages, namely the way they physically store arrays. The FORTRAN standard specifies column-major order (i.e. first index is least significant) when physically storing arrays in memory. $C$, on the other hand, specifies row-major order (first index most significant) when storing the arrays. If a FORTRAN program were to pass a multi-dimensional array to a $C$ function, the array would have to be first transposed. Then, the array is used by the $C$ routines, and again transposed before being passed back to the FORTRAN program. The translator emulates the physical storage of the FORTRAN code by collapsing all n-dimensional arrays to a single dimension, and calculating array indexes internally. The layer code performs all necessary transpositions when building the equivalent $\mathrm{C}$ structure.

\subsection{Ubiquitous Subroutines}

In this section, we discuss briefly the function of some of the more commonly-used subroutines. Since these are used throughout the PVT system, anyone wishing to understand the code should be familiar with their purpose. There are many other subroutines, however. All PVT subroutine libraries are self-documented, so when questions arise, the code itself should be consulted.

\subsubsection{Initialization And Clean-up of Test Programs -}

Normally, the first executable statement within a program will be a call to INITGL. This routine performs all the work needed to set up the environment for the program; in particular, it initializes the values in the common areas GLOBNU and GLOBCH so that they may be used freely (e.g. workstation type needed to open a workstation). It does this by reading certain constant values from the PVT configuration file (see section 4.2 on Running INITPH). The program supplies its identifying TEST NUMBER (see section above on Banner) as the single parameter.

For interactive programs, the SETDLG subroutine sets up the dialogue area as requested in the configuration file, and initializes the DIALOG common area. 
The last executable statement is a call to ENDIT. This closes all open workstations, closes the currently open structure, and closes PHIGS. It then calls WINDUP to write out summary results, close files, and perform any other processing needed to finish the test program.

\subsubsection{TC Subroutines -}

There are a number of subroutines needed to implement a given TC in the code. These correspond closely to the way a TC is set up in the pseudo-code. The SETMSG subroutine sets up a "current TC message" for the condition about to be tested, which contains both the SR references and the text describing the condition under test. If a test case is currently being checked for adherence to the standard, the test message will include "[UNDER REVIEW]".

Depending on the results of the $\mathrm{TC}$, the program then executes either the PASS or FAII subroutine, which records the result. The IFPF subroutine is a shorthand form, which accepts a single logical expression as a parameter and invokes PASS if it evaluates as true, and FAIL if false.

\subsubsection{Message Subroutines -}

The only persistent output of each test program is a series of messages. The following subroutines generate messages of the indicated type:

\begin{tabular}{lll} 
Subroutine & Message-type & Function \\
\hline INITGL & SY & initialize program \\
ENDIT & SY & finalize program \\
PASS & OK & record TC passed \\
FAII & EA & record TC failed \\
IFPE & OK or FA & record TC result \\
INMSG & IN & information \\
UNMSG & UN & abort \\
NCMSG & NC & abort \\
CHKINQ & NC & continue or abort
\end{tabular}

See section 4.4 .6 on for the interpretation of messages and message-types. The subroutines UNMSG and NCMSG are used when the program must be aborted, as opposed to normal program conclusion which is done via ENDIT. No further code is executed after either of these is encountered in the flow of control. 


\section{7 .4 CHKINQ -}

It is very common within the PVT system for an inquire function to be incidental to the main purpose of the test. Since the test relies on the result of the inquire, we wish to ensure that it has completed successfully. Every incidental use of an inquire, therefore, is followed by CHKINQ, which simply checks that the error indicator from the function is zero. If not, CHKINQ invokes NCMSG and therefore aborts the program. Otherwise there is no effect.

of course, when an inquire function is being purposefully tested, its error indicator is checked explicitly as part of the usual PASS/FAII determination.

\section{INSTALLATION}

This section covers the steps needed to install the PVT code on a typical computer system. The advice is necessarily general, since many aspects of installation are system-dependent. Installation consists of the following steps:

1. Copy files into hierarchical directory

2. Customize source code, especially naming of configuration file

3. Compile all subroutines

4. Optionally, set up batch procedures for passive programs

\subsection{File Storage}

Copy the PVT files from the medium on which they are delivered to the hosting system. If the medium is magnetic tape, there is an accompanying information sheet to describe the physical format of the files. Also, see Appendix G for detailed guidance on creating the $C$ version under UNIX.

\subsubsection{Hierarchical Format -}

Store the files in a hierarchical directory as described in section 2.1 and Appendix B. In particular, use the same two-digit level numbers as in the files' own self-identification. The name of the PVT root level is arbitrary; a name like USER PVT ... is a reasonable choice. See section 2.1 for a list of the files that belong in the PVT root directory. The global and module-level 
documentation need not be stored on-line, although this may be convenient for automatic searching for entities within the PVT suite.

Assign the source code filenames in the format "pxx.f" where " $\mathrm{xx}$ " indicates the ordinal number within the module, and ".f" indicates the source language. For example, the program with the header

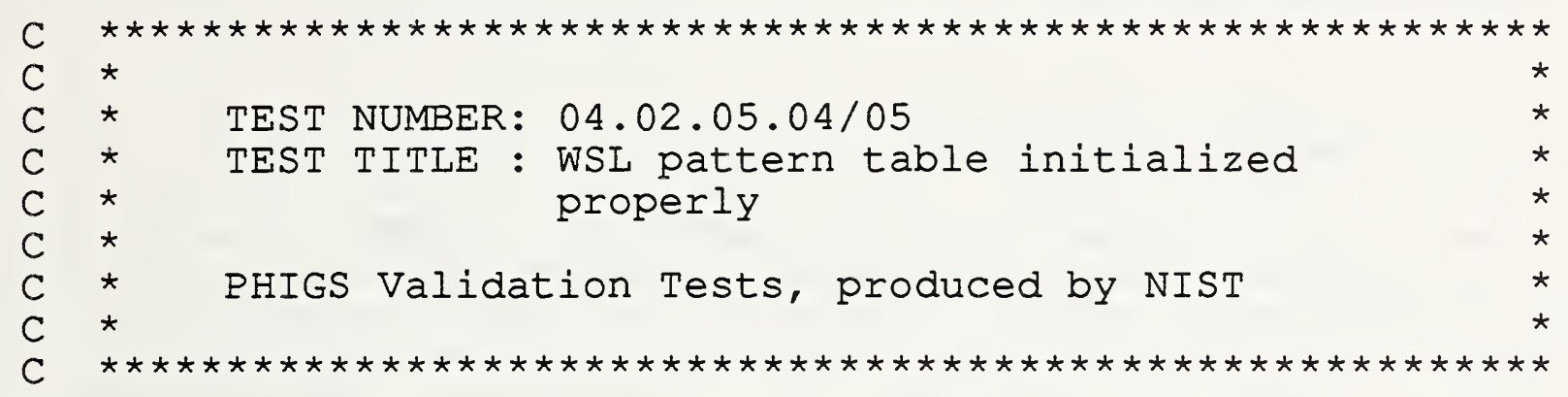

should be stored as "user pvt 04 02 05 04 p05.f". Different operating systems have various conventions for the suffix to denote Eortran source code, such as ".FOR" or ".FTN". Any such convention is acceptable as long as it is consistently applied. It is strongly recommended, however, that the initial part of the name be in the format "Pxx". Throughout the PVT documentation, programs are always identified according to this format, and it avoids confusion if the program's physical name matches its logical identification.

Iikewise, copy subroutine libraries into the appropriate tree nodes. The banner of each subroutine identifies the correct node for the library. The recommended file name is sublib.f (Fortran) or sublib.c (C).

When done, check your file hierarchy against the PVT tree structure as described in appendix B to ensure that all the files have been copied.

\subsubsection{Alternative Storage Strategy For Subroutines -}

The local subroutine libraries are assigned to nodes of the tree only for the purpose of clarifying the logical relationship among the test programs and subroutines. If linking in several libraries is difficult in your system, you can simply concatenate all the subroutine source code together as one large file, and compile it as one library, presumably in the root. No two PVT subroutines have the same name, so no name clashes will occur.

This alternative method is also on the distribution tape for the $C$ binding under the PVT root, pvt V2LIB (see Figure 3). This may allow easier generation of object libraries for some operating systems. The sub-directory pvt/V2LIB contains all routines, one 
routine per file, each file name being the routine name. Files ending in . $C$ are the $C$ versions translated from the equivalent FORTRAN versions (e.g. XPOPPH.c was translated from XPOPPH.f). Each of the files must be compiled and assembled into a library for linking. All object files resulting from the compilation of source files ending in . C should comprise a single library. It is not recommended that the files be separated into multiple libraries because many operating systems require that the calling order of the routines be followed.

\subsection{Customization of Code}

Some of the source code may need to be changed in order to run on your system. These places are noted in the code by a comment line beginning with "CMOD". Use any text editor to perform these changes. The first change, naming the PVT configuration file, is mandatory for all systems. All the other changes are optional.

\subsubsection{Naming PVT Configuration File -}

First, choose a name for the PVT configuration file. The name you pick must be absolute, i.e. it must be valid when used from any part of the hierarchy. We recommend locating the PVT configuration file in the PVT root.

This name must be inserted in three locations all of which are in the PVT root:

1. the INITPH program which writes the file

2. the INITGL subroutine which reads the file and is located in the global sublib file

3. the MULTWS subroutine which reads the file, also located in the global sublib file

In all three cases, search for the string "INITPH.DAT" (the name we used) in these routines to locate the insertion point.

The PVT configuration report file is a human-readable version of the PVT configuration file. Pick a name for it as well, such as "INITPH.PRT", and insert it into the INITPH program.

3.2.2 Special Processing For Opening PHIGS - 
All the test cases call XPOPPH to open PHIGS, rather than the standard POPPH. The XPOPPH subroutine, as delivered, simply calls POPPH. If your system has special processing requirements for accessing PHIGS, these may be addressed by additions to XPOPPH. Note, however, that for validation purposes, such changes are subject to approval by NIST.

\subsubsection{Random Number Generator -}

The last routine in the global subroutine library, RND01, is written in non-standard Fortran, since that standard provides no way to randomly initialize a seed for a random number generator. You may alter RND01 (possibly using a local non-standard time function) so that the seed is different for each execution. This is the preferred mode of execution. All the other random-number routines are built on RND01, so if it works, the others need not and should not be changed.

For the $C$ version, the standard C function, gmtime is used to obtain a random starting value, and so no modification should be necessary.

\subsubsection{Naming Individual Message File -}

If you request that the test programs generate individual message files (see section 4.2.3) INITGL will, by default, form the name of the file by using "p" as a prefix, the two-digit ordinal number of the program, and ".msg" as a suffix; e.g. p04.f will write to p04.msg. if you prefer another naming convention, search for ".msg" in the INITGL routine and change the code accordingly.

\subsubsection{Resolution of Parameters For <open workstation> -}

The INITGL and MULTWS subroutines read the PVT configuration file in order to determine the parameter values needed to open the primary and secondary workstations (workstation identifier, connection identifier, and workstation type), and report these back to the calling program. INITGL sets the value of three variables in COMMON to do this, while MULTWS returns the values in its output parameters. In both cases, the assumption is that the correct values are static and can be set once by the INITPH procedure (see section 4.2 , below). If your system is such that this information can be determined only at run-time, you must re-code the relevant sections of INITGL and MULTWS, so that they still deliver the required values. 


\subsubsection{Providing Valid Names For Archive Files -}

The subroutine AVARNM, in node 03 of the PVT tree, must return to the caller an integer representing the valid name of an available empty archive file. The code assumes that this name should be interpreted as a Fortran logical unit number. If your system has a different interpretation, or has special requirements for opening an archive file, you must modify this subroutine accordingly.

\subsubsection{Time-stamping Message Files -}

The Fortran standard provides no function for determining time or date. If, however, your implementation does provide such a feature and you wish to include this information in the PVT output, we suggest you alter the INITGL subroutine at the point where it formulates the header system message. This is done at the very end of the subroutine, in the last call to BRDMSG.

If you are modifying the c version, you may wish to use one of the standard $C$ functions that return the current time.

\subsubsection{Operator Communication -}

The OPMSG and OPYN routines in the global sublib.f library write messages to and read messages from the operator. Because a workstation may not be open at the time these are executed, the PVT Fortran code resorts to the use of the print and read statements. If there is a better way to send a character string to and from the operator in your system, you may re-code these routines accordingly. If print and read work well within your system no change is necessary.

\subsubsection{Appending To Global Message File -}

If you specify a global message file (see section 4.2.3), the INITGL routine must position the file pointer at end of file so as to append new messages. In standard Fortran, the only way to do this is to read through the whole file. If your Fortran system provides a more efficient (though non-portable) way to do this, you may wish to substitute that method in INITGL. Otherwise, no change is needed.

Since the translator uses the FORTRAN code, this inefficiency is carried into the generated $C$ code. If you wish to provide a more efficient way in $c$, such as opening the file in append mode, you may substitute the method for the one provided in INITGL. 
Since ANSI compliant $C$ allows the use of prototyping [C1989], the layer code for the $C$ binding provides for both non-prototyped and prototyped source code. The use of a define directive in each source file regulates which function declaration will be used. If your complier supports prototyping, no define directive need be used. If your complier does not support prototyping, all code must be compiled with the directive NO PROTO. This may be done by changing the "Makefiles" in the PVT root, in V2LIB, and in the LAYER directories.

\subsubsection{Special Characters -}

The test suite makes use of the entire printable ASCII character set (one of the requirements of the PHIGS standard for the <text> function). The characters appear in the source code and may also have another meaning on some systems (e.g. the backslash "l" character in UNIX). Most compilers have a switch/parameter that notifies the compiler to ignore the special "system" meaning of these characters during compilation. If the code does not compile, check if your system uses any special characters, and the switch/parameter for your compiler that disables them. If that doesn't work, the source code itself may have to be modified (e.g.

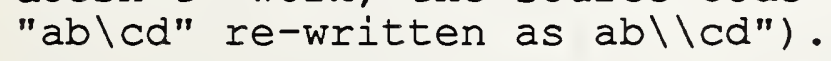

\subsection{Compile Subroutines}

In most systems, you will need to compile all the subroutine files so as to create subroutine libraries, which may then be linked in with each test program. Test suite programs for both language bindings require:

1. errlib, trans-sublib and sublib in the root

2. all the local sublib libraries in current and ancestor nodes, or in the case of the $C$ binding and single directory method, access to the V2LIB node

3. the PHIGS library under test, and possibly other implementation dependent libraries, such as those to support windowing systems

The $C$ binding also requires:

1. the f2c (FORTRAN to C) subroutine library, conventionally named libf2c. 
2. the appropriate C libraries to support mathematics and clock functions

Of course not all the test programs use all the subroutines, so do whatever your system requires such that only needed subroutines are linked in. E.g. in VMS, the /LIBRARY qualifier performs this function.

We strongly recommend setting up a command procedure to compile, link, and execute a test program solely by referring to the name of the program. In particular, all local subroutine libraries (those above the program in the directory tree) and the global libraries must be made available, as well as linking to the code of the PHIGS implementation. Note that errlib.f contains the PERHND subroutine - the standard PHIGS-Fortran name for an error-handler. Correspondingly, errlib.c in the $C$ version contains perr hand - the standard PHIGS-C name for an error-handler. Some PHIGS implementations may require special treatment to link in PERHND or perr hand. Since most linkers prefer to be given access to libraries in order from caller to called procedure, the normal sequence would be:

1. the local PVT libraries,

2. trans-sublib

3. the global PVT library, sublib

4. errlib (unless the system error-handler is to be used)

5. layer code for $\mathrm{C}$ binding

6. $\mathrm{f} 2 \mathrm{C}$ library

7. PHIGS library

8. support libraries for C language

See Appendix $F$ for two examples of how this might be done. These examples also show how errlib can be included or not: this is the way that many systems control whether a user-defined or system-defined routine performs error handling.

\subsection{Procedures Eor Batch Processing}

It may prove useful to construct a batch procedure (such as a shell script in Unix) for the passive tests of each category of workstation, so that you can automatically invoke the correct subset of programs. Be careful, however, that you provide for the possibility of non-completion (e.g. failure to compile, aborted execution) of programs run in batch mode. 
In this section, we cover the steps needed to run and interpret the PVT system. Like other conformance test systems, the PVT is not, nor can it be, a totally automated process. The PVT code and documentation are best seen as components of an integrated and interdependent system, which includes the operator as its active, directing component. We include in the notion of operation the process of interpreting the behavior of the programs, particularly with regard to conformance.

\subsection{PVT Sessions And Workstations}

Let us refer to the execution of a set of PVT programs as a session. For each session, a primary workstation must be specified, together with an optional set of secondary workstations. The primary workstation is the one whose features are thoroughly tested by the PVT programs. Secondary workstations are tested only by certain special-purpose programs such as those dealing with multiple workstations. Thus, for each workstation to be tested in depth, the operator must run a distinct session.

In order to conform, a PHIGS implementation must support at least one workstation of category OUTIN. No other workstations need be supported. If other workstations are accessible, however, they must have the capabilities associated with their categories.

Thus, a typical scenario for conformance testing is first, a session in which an OUTIN workstation is designated as primary and in which all the PVT programs are executed. Such a session checks the minimum requirements for conformance.

Subsequent sessions deal with the capabilities of other workstations of whatever category. Each of these workstations is designated in turn as primary and an appropriate subset of the PVT is executed for it. Since there is no need to re-run tests which are not relevant to the capabilities of these other workstations, not all PVT programs need be executed in these subsequent sessions. Use the information in the CHARACTERISTICS entry of the program design documentation (summarized in appendix $D$ and in the pgm-char.prt file) to decide which programs are pertinent, according to the table below.

Workstation

Category

OUTIN

OUTPUT

INPUT

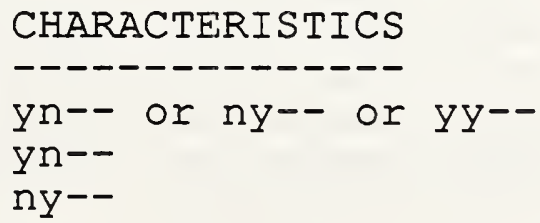


Programs with a CHARACTERISTIC entry of "-nn" require no operator interaction and may be run in batch mode; otherwise there must be an operator for the test. By convention, within a module, all the passive programs precede the interactive ones (e.g. P01-P05 are passive, P06-P09 are interactive).

Figure 4 summarizes the way in which several sessions are performed so as to constitute a complete test of conformance.

for each PHIGS implementation (hardware platform + software)

for each language binding (Fortran, C)

INITPH for primary OUTIN workstation

execute all PVT tests for this language

use COMPIT to check completeness of session

for each additional workstation type

INITPH for this workstation type

execute workstation PVT tests of appropriate category

(OUTIN, OUTPUT, INPUT) for this language

(see PGM CHAR.PRT file for guidance)

use COMPIT tó check completeness of session

next workstation type

next language binding

next implementation

Figure 4: Use of PVT sessions to test conformance

\subsection{Running INITPH For PVT Configuration}

The PVT Configuration file contains the information which is specific to the PHIGS implementation being tested, but the same for all test programs within the PVT session. Its purpose is to allow an operator to specify such information only once at the beginning of each session, rather than repeating it for each program. The INITPH program creates this file, based on the operator's responses. The program is stored as initph.f or initph.c in the PVT root. It uses some subroutines from the global subroutine library, and also from PHIGS itself, so these libraries must be available as it is compiled and linked. 
Execute INITPH, and respond as prompted. Most responses are in the form of an integer. When parameter values are requested, they should be supplied as needed by the Fortran binding. Conversion to $\mathrm{C}$ will be done automatically by the system. E.g. the error file parameter to <open phigs> is supplied by you as an integer, not a character string. You should be prepared to supply the following information to INITPH:

1. parameters for <open phigs> (error file and memory units)

2. number of workstations accessible in this session

3. Copen workstation> parameters for each accessible workstation (workstation identifier, connection identifier, workstation type)

4. whether to suppress "pass" messages

5. choice of destination(s) for messages (screen, individual files, or global file)

6. file name and logical unit for global message file (if used)

7. logical unit for individual message files (if used)

8. maximum line length for messages

9. whether the implementation allows the error file to be explicitly opened and named by the application program prior to execution of <open phigs>

10. an identifying character string contained in error messages generated by the system

11. whether to control the tests with a pseudo-random or true random number sequence

12. whether the primary workstation is capable of visual output

13. method for prompting the operator, and maximum line length for prompts

14. method for operator responses to prompt

15. location and size of the dialogue and echo areas

16. ratio of meters to DC units for the primary workstation (to be supplied by you explicitly, or to be measured on screen) 
The first two questions concern the input parameters to be passed to the <open phigs> function, whenever that function is needed in a PVT program. Simply supply the numeric values your implementation requires.

\subsubsection{Parameters For Opening Workstations -}

The next questions concern the workstations accessible to this implementation. Tell INITPH the total number of accessible workstations (primary and secondary). Then, for each of these, supply the three parameters by which <open workstation> can open it: workstation identifier, connection identifier, and workstation type. Be sure that the first set of parameters refers to the primary workstation.

\subsubsection{Control of Messages -}

Next, INITPH will ask you whether you want a message to be generated whenever the implementation successfully passes a TC in a test program. You can specify either that such messages are always suppressed, always generated, or that each program asks the operator what to do, so that he or she can selectively suppress pass-messages at run-time. No other type of message can be suppressed.

The next questions deal with the destination of messages as they are generated. First, indicate whether or not messages are to be sent to the operator (typically on the screen). Next, indicate to which files messages should be written. Individual message files are created once per execution of a test program. By default they are given the same name as the program, but with a "msg" suffix, rather than "f". The global message file is a cumulative file to which messages are appended whenever a test program is run.

These are independent choices; messages can be sent to any combination of the three destinations: operator, individual file, or global file. Each enabled destination receives exactly the same set of messages.

If you specify a global file, you must then provide an absolute name for this file, so that all programs can write to it. You may want to specify a distinct name for the global message file of each PVT session. This response is, of course, not in the form of an integer, as are the others. Since some operating systems have reserved logical unit numbers in Fortran, you are also asked to provide these for the individual and/or global file, if they have been designated as destinations. 
You must specify the maximum number of characters per line which should be generated when the PVT system formats a message. some messages may be quite long and would not fit on a reasonably-sized single line. Message text is never truncated; rather, it is simply broken into lines of the specified size.

\subsubsection{Control of Error File -}

Many of the PVT programs that test error handling do so by reading the error file created by the implementation. If possible, the ESETUP subroutine in 09.01 will assign the name "pxx.erf" to the error file generated by the program pxx.f, by explicitly opening the file and then passing its logical unit number to <open phigs>. Otherwise, the system default name is used. Tell INITPH whether your implementation allows opening of the error file prior to <open phigs>.

When the PVT attempts to read and examine the error file, it does not assume that there is exactly one record per error message. Therefore, if each message contains a special character string (such as "PHIGS ERROR:"), supply this value so that multi-record error messages cañ be distinguished automatically.

Finally, INITPH will attempt to automatically create an error file with a single error message; the text of this message will be used as a separator when later tests analyze the contents of error files generated by the system. Therefore, this single-message error file must be readable by those tests, under the name "errsep.erf". If the error file cannot be explicitly named by the program, you must re-name it after execution of INITPH from its system name to "errsep.erf". INITPH will display a message if this is necessary.

\subsubsection{Control of Randomization -}

Many of the interactive tests randomize the choices presented to the operator so that the correct responses are not predictable (see section below on operator interaction). For some purposes, however, it is desirable that the tests execute with repeatable displays and operator prompts. If you want to get repeatable behavior, enter a real number between 0.1 and 0.9 . This value will be used as the seed for a random number sequence. Thus, re-initializing to a distinct value between 0.1 and 0.9 will cause repeatable behavior within the new session, but distinct from that of the previous session.

Entering any value outside the range of $0.1-0.9$ causes the system to use a time function to set the seed for the random sequence, and thus generates truly random operator choices. 
4.2.6 Primary Workstation Support For Graphical Output -

If the primary workstation is capable of visual output, answer yes, otherwise no. The normal answer is "yes". The question is here to allow for testing of INPUT-only workstations and metafile workstations in later versions of the PVT.

\subsubsection{Control For Prompting The Operator -}

When running the interactive tests, the system poses questions to the operator. This option lets you choose the mechanism for transmitting those questions: 1-Fortran print (or its C equivalent), 2-PHIGS <message>, or 3-PHIGS <text>. In many systems, Fortran PRINT will write to another window. PHIGS <text> will use some of the PHIGS display space for questions, possibly leaving less room for the picture under examination.

If using Fortran print or PHIGS <message>, you must specify the maximum number of characters per line in interactive prompts.

\subsubsection{Control For Operator Responses To Prompt -}

You may specify the means by which the operator is to respond to prompts: either $1-$ Fortran read (or its $C$ equivalent), or 2-PHIGS <request string> (and the device number for <request string $>$ ).

\subsubsection{Location And Size of The Dialogue And Echo Areas -}

If you are using PHIGS for operator input or output, you must specify where the dialogue area (containing operator prompts and responses) should be. The choices are: 1-dialogue at bottom of screen or PHIGS window, 2-dialogue at right, or 3-toggle picture and dialogue so that the screen can alternate betweem the two. Since the picture area will use the largest square remaining on the screen after the dialogue area has been reserved, it is recommended that the dialogue area be on the right for wide screens, and on the bottom for tall screens.

You must then specify what percentage of the screen should be reserved for the dialogue area. Something in the range of 15-30 percent is usually a reasonable choice.

Finally, if you are using PHIGS for operator input, specify what percentage of the dialogue area is to be reserved for the echo of operator responses (the remaining area is used for prompts). Since prompts are usually larger than the responses, something like 10-20 percent is a good choice. 
4.2.10 Ratio of Meters To DC Units For The Primary Workstation -

You must tell the system the ratio of meters to DC units for this workstation. You may either enter the number directly, or physically measure a diagonal line on the screen, and INITPH will compute the result.

At the successful conclusion of INITPH, the operator receives a report on the names of the files to which the PVT configuration file and PVT configuration report file (the human-readable version) have been written.

\subsection{Background Documentation}

In order to understand the significance of the programs'
output for conformance, you should read over the module documentation and those sections of the standard referred to in the \#S entries of the SRs. You will then have the appropriate background information to allow you to focus on the details of each test result.

\subsection{Execution And Interpretation}

Invoke the system-dependent command(s) needed to compile, link and execute each program in the session. There are a number of possible outcomes, each of which are dealt with below. We assume throughout the following sections that the PVT programs are themselves valid. If you encounter some aspect of a program which you believe is incorrect, please submit a comment to that effect as described in section 1 .

\subsubsection{Testing Error Handling -}

Normally, test programs are executed with the error handler defined by the PVT (residing in errlib in the PVT root) in effect. Those programs, however, that test the behavior of the system error handler may have to be invoked in a different way, so as to exclude errlib. These programs include all those in modules numbered $09.01 . x \times .02$, and 09.01/p03-p05.

\subsubsection{Language Specific Tests -}

Note that some modules pertain only to a specific language binding. For version 2.1 of the PVT these are:

$$
\text { Fortran only: C only: }
$$



09.01 .10
09.01 .11
09.01 .10 .01
09.01 .11 .01
09.01 .10 .02
09.01 .11 .02
09.02 .10
09.02 .11
11.01
11.02

\subsubsection{Failure To Compile or Link -}

If your compiler does not support some feature of ANSI Fortran [FORT78] or ANSI C [C1989] used by a PVT program, you must find a different way to perform the equivalent operation. Note, however, that such lack of support constitutes non-conformance, since the Fortran binding [PHFOR90] incorporates the Fortran standard [EORT78] and the C binding [PHC91] incorporates the C standard [C1989]. Similarly, problems in the system software which performs linking of subroutines must be resolved by you. Any problem with system software which prevents a valid invocation of a PHIGS function by a program renders the implementation non-conforming.

Uncorrected failure to begin execution of a program should be noted by running the OPRCMT program. This will record operator comments as messages in the specified destination files. Therefore the results of all tests (even non-executing tests) are saved in a uniform way. Even though the OPRCMT program resides in the PVT root, you should be in the sub-directory of the appropriate module when executing OPRCMT so that the individual message file will be written in the correct location. For example, suppose program 06.01.02/01 cannot be executed. Then, while still in that sub-directory, execute OPRCMT, enter the identifier of the module and program ("06.01.02/01"), and then enter your comment(s) as prompted by the program.

\subsubsection{Failure To Complete Execution -}

The PVT programs are written conservatively so as to fall well within the operational limits of any reasonable PHIGS implementation. Furthermore, the PERHND (or perr hand, for C) subroutine supplied in errlib.f (or errlib.c) should handle any errors signalled by PHIGS functions. Therefore, it is to be expected that every PVT program, once started, will proceed until completion under the control of the appropriate PVT subroutine, such as ENDIT for successful completion, or UNMSG for aborted completion.

If the PHIGS system itself aborts execution, it means that the implementation fails the test and does not conform, since no unhandled PHIGS error conditions should arise within the PVT code. If not otherwise evident, this outcome is indicated by the absence of two sY-type messages (the first containing the error count, the 
second to identify PVT completion) at the end of the message file (s).

You may wish to use the OPRCMT program (see preceding section) to enter an operator comment on the cause of failure, but be aware that the individual message file generated by the original program may be over-written. The cumulative message file will contain the output of both the aborted program and OPRCMT.

\subsubsection{Operator Interaction -}

The interactive tests all present a graphical display to the operator in the picture area of the screen and then ask him/her a question about it. See the OPERATOR SCRIPT entries in the DOC.TXT files for detailed instructions if the screen is not self-explanatory.

\subsubsection{Format Eor Answering Questions -}

In general, the format of the response should be obvious from the question. Most questions are single integer choices, such as "Which fill area is different?" referring to a display of perhaps 8 numbered fill areas. Answer such questions with a single integer, in the example given, 1 through 8 . If there is no unique correct answer (e.g. all fill areas are the same, or several differ from each other, or the screen is blank) respond with "0" (see below).

When a list is called for (e.g. "Which primitives are highlighted?"), enter a list of integers separated by commas, e.g. $" 2,3,5,6 "$. If the order of the list is significant, the prompt will. so indicate. The single character " $n$ " denotes the empty list (e.g. your response should be " $n$ " if none of the displayed primitives were highlighted).

Other response types are: real numbers, "y" or " $n$ " for yes/no questions, and character strings. If a response has an incorrect format, PVT issues a warning and lets you re-enter the response.

\subsubsection{Special Processing of Operator Responses -}

Except for yes/no questions, which require " $y$ " or " $n$ ", a response of "O" is always valid and means that the question is unanswerable. After a "o" response, the operator is given an opportunity to enter a free-form comment explaining the nature of the problem. The comment is then recorded in the output message file. An operator comment is entered as several text lines, and terminated by a line containing a single period. If the first line of the comment is the termination line, no message is generated. 
Usually a "0" response causes the associated test case to fail.

Also, the operator may respond to any prompt with "d" (at-sign). This allows him/her to enter a comment pertaining to the current picture and prompt, but without causing failure as would the "0" response. The comment is made before actually answering the question. As with "0", the comment is recorded as a message and terminated by a line with a single period. After entering the comment, the original question is put to the operator again and must still be answered.

When the operator has indicated to INITPH that the dialogue and picture area are to be toggled (see section 4.2.7), the toggling is controlled by entering "t" or "T". The effect is to switch between displaying the dialogue area and the picture area. The actual answer may be entered when either area is visible.

\subsubsection{Completion of Execution And Message Type -}

The PVT programs sometimes examine whether they can proceed to execute, or whether anomalous conditions have arisen such that further execution is futile. In the latter case, before stopping, the program will issue a message of type UN or NC, indicating the reason for aborting further testing. Normally, all the TCs within the program that are appropriate for the implementation are executed.

In either case, the output of a completed PVT program consists of a sequence of messages. These messages are sent to the destinations specified in the PVT configuration file. They are followed by three records each consisting of a single period. This serves to separate visually the output of each test in the global message file.

The messages are categorized into six types in order to help the operator assess their significance. The output is formatted so that these types appear in the leftmost columns, with the text of the messages indented. The following table describes the circumstances associated with each of the message types.

\section{Message Types}

SY: System message, generated by INITGL and ENDIT. These messages herald the beginning and completion of execution of a PVT program. The next-to-last message contains a summary of the number of TCs executed and failures detected.

OK: The standard message resulting from a passed test case, generated by PASS or IFPE. INITPH allows the operator to suppress messages of this type. 
FA: The standard message resulting from a failed test case, generated by FAIL or IFPF.

IN: Informational message, generated by INMSG or SIGTST. Some situation was encountered which, while not erroneous, is worth noting, for example, the implementation is such that a certain condition cannot be tested. Also, this type of message can be used for the purpose of conveying useful but not conformance-related information about the implementation, such as support of optional PHIGS features.

OP: Message as entered by the operator, either as a result of entering "0", or "e" in response to a prompt. Typically, this message is used to record some unusual or unexpected visual feature of the display.

UN: Unanticipated error message, generated by UNMSG. This message is generated when the program detects some anomalous condition that prevents further processing. No inference is to be drawn concerning implementation conformance.

NC: Unanticipated non-conformance error message, generated by NCMSG or CHKINQ. The program must abort because a feature of PHIGS which is not under test, but needed for the test, has failed. Since the incidental feature is mandated by the standard, its failure indicates non-conformance. Most commonly, this message type is generated by CHKINQ when a required inquire function fails.

\subsubsection{Analysis of Results -}

The operator may inspect the output at several levels of detail. In the simplest case, he or she may simply look at the error count at the end, to see whether or not all tests were passed. By scanning the left column for FA or NC type messages, the operator can focus on problems of non-conformance and ignore the other output. Finally, one can note the content of informational messages, which tests were passed or failed, which SRs are implicated in the result, and what the precise conditions of the test were.

\subsubsection{Troubleshooting -}

Obviously, these is no foolproof procedure for analyzing unexpected results, but we recommend the following steps: 
1. Look at the design for the program in question contained in the file "DOC.TXT" in the same sub-directory. This document describes the part of the standard that is being tested and the methodology the test is using.

2. In the case of Fortran, read the code as it corresponds to the design. Subroutines may be located via the subroutine directory in Appendix E.

3. As previously stated, the $\mathrm{C}$ code is the translated version of the equivalent FORTRAN code. The converter translates the FORTRAN source code, but much of the readability of the original code is lost in the translation. If you have both language versions, it may help to read the equivalent Fortran alongside the $\mathrm{C}$ code.

4. If the failure occurred on a PHIGS call in the $C$ version, check the source code for the layer routine that failed. This code resides in the sub-directory pvt/LAYER (see Figure 3).

\subsection{Session Completion}

When you believe you have completed a session you may use the COMPIT utility to examine the global message file produced by the session so as to check that no programs have been skipped. COMPLT does not work in the absence of a global file. This utility is a simple Fortran or C program; it does not call PHIGS.

As prompted, enter the name of the global message file to be checked, whether the file was produced by the Fortran or c binding, and the expected subset of the PVT, based on the category of the primary workstation. COMPLT will report any discrepancies between the expected subset of the PVT and the actual subset executed, as reflected by the presence of "End execution" messages in the global message file. The OPRCMT utility generates the "End execution" message expected by COMPLT; therefore invoking OPRCMT for any programs in the session that have failed to run to completion is required in order to preclude error reports from COMPLT.

\section{6 Customization For Debugging}

Users who are interested in debugging as well as conformance checking may wish to augment the tests so as to produce more detailed information. We recommend that the extra output from such enhancements follow the usual conventions of the PVT system. In particular, we recommend that the information be formatted into a character variable (e.g. using Fortran's WRITE statement) and then written out as an IN type message with the INMSG subroutine. There 
are many examples of this in the normal code. See these if you need further guidance. 
[CUGI90] John Cugini, Mary T. Gunn, Lynne S. Rosenthal, PHIGS Validation Tests (Version 1.0): Design Issues, NIST SP 500-181, National Institute of Standards and Technology, Gaithersburg, MD, 1990 .

[CUGI91] John Cugini, Interactive Conformance Testing for PHIGS, Eurographics ' 91 (ed. F. H. Post and W. Barth), Elsevier Science, New York, NY, 1991.

[C1989] Programming Language C, ANSI X3.159-1989, American National Standards Institute, New York, NY, 1989.

[FORT78] Programming Language FORTRAN, ANSI X3.9-1978, American National Standards Institute, New York, NY, 1978.

[GKS85] Computer Graphics - Graphical Kernel System (GKS) Eunctional Description, ANSI X3.124-1985, American National Standards Institute, New York, NY, 1985.

[GKST89] GKS Validation Test Suite, Version 2.1, National Institute of Standards and Technology, Gaithersburg, MD.

[PHFOR90] Information processing systems - Computer Graphics Programmer's Hierarchical Interactive Graphics system (PHIGS) language bindings - Part 1: FORTRAN, ISO/IEC 9593-1:1990, American National Standards Institute, New York, NY, 1990.

[PHC91] Information technology - Computer Graphics Programmer's Hierarchical Interactive Graphics system (PHIGS) language bindings - Part 4: C, ISO/IEC 9593-4:1991, American National Standards Institute, New York, NY, 1990.

[PHIGS89] Computer Graphics - Programmer's Hierarchical Interactive Graphics system (PHIGS) (Part 1: Functional Description), ISO/IEC 9592-1:1989, American National Standards Institute, New York, NY, 1989. 
APPENDIX A

GLOBAL VARIABLES

Below is a description of the variables in COMMON in the Eortran source code. They are used globally throughout PVT.

\begin{tabular}{|c|c|c|c|}
\hline Variable & \multicolumn{2}{|c|}{ Type* } & Description \\
\hline CONID & S & $I$ & Connection identifier, for <open workstation> \\
\hline CTLHND & D & $I$ & Tell PERHND whether to abort $(0)$ or return (I) \\
\hline DUMCH & S & $\mathrm{C}$ & Dummy character variables for future use \\
\hline DUMINT & $\mathrm{S}$ & $I$ & Dummy integer variables for future use \\
\hline DUMRI & $\mathrm{S}$ & $\mathrm{R}$ & Dummy real variables for future use \\
\hline ERRIND & $\mathrm{D}$ & I & Error indicator, returned from inquire functions \\
\hline ERRFIL & S & I & PHIGS error file, for <open phigs> \\
\hline ERRSIG & D & I & Signalled error code returned by PERHND \\
\hline ERRSW & S & $I$ & Indicates whether to send messages to operator \\
\hline FUNCID & $\mathrm{D}$ & C & Function signalling the error, returned by PERHND \\
\hline GLBERR & $\mathrm{s}$ & C & Absolute name of global message file \\
\hline GLBLUN & $\mathrm{S}$ & $I$ & Logical unit number of global message file \\
\hline IERRCT & $\mathrm{D}$ & $I$ & Count of errors detected by test \\
\hline IFLERR & $\mathrm{S}$ & $I$ & Controls writing of messages to message files \\
\hline INDLUN & $\mathrm{S}$ & $I$ & Logical unit number of individual message file \\
\hline MAXLIN & $\mathrm{S}$ & $I$ & Maximum characters per line in messages \\
\hline MEMUN & $S$ & $I$ & Number of memory units, for <open phigs> \\
\hline PASSSW & $S$ & $I$ & Controls writing/suppression of pass-messages \\
\hline PIDENT & $\mathrm{D}$ & $\overline{\mathrm{C}}$ & Unique program identifier - hierarchical number \\
\hline TESTCT & D & I & Count of conditions tested so far within program \\
\hline TSTMSG & D & $\mathrm{C}$ & Text for next condition to be reported. \\
\hline UNERR & $\mathrm{D}$ & I & Count of unanticipated errors detected by test \\
\hline WKID & $S$ & $\bar{I}$ & Workstation identifier, for copen workstation> \\
\hline WTYPE & $\mathrm{S}$ & I & Workstation type, for <open workstation> \\
\hline
\end{tabular}

* S: Static value read from configuration file

D: Dynamic variable, altered during test execution

I: Integer
$\mathrm{C}:$ Character
$\mathrm{R}:$ Real 

APPENDIX B

PVT TREE STRUCTURE

This appendix describes the tree structure of the PVT system. The status of each node of the tree is specified in the left column, and is one of the following:

a. Node does not contain a module. Denoted by "-".

b. Node contains a module. Denoted by a positive integer, $n$, indicating the number of programs in the module. The node contains exactly one DOC.TXT file, and $n$ program files, numbered 1 through $\mathrm{n}$.

c. Future module(s); the node, or its descendants will contain modules in future versions of the PVT. Denoted by "f".

The second column indicates whether the node contains a local subroutine library, named sublib.f, to be used by modules within that node's subtree. If so, an " $s$ " appears in the column. 
0101 Opening and closing PHIGS

02 Manipulating the CSS

$03 \mathrm{~s} 02.01$ Creation and inquiry of CSS networks

$10 \quad 02.01 .01$ Individual structure creation

- 02.01 .02 Structure deletion functions

$06 \quad 02.01 .02 .01$ Individual structure deletion

14 s 02.01 .02 .02 Structure network deletion

$02 \quad 02.01 .02 .03$ Global structure deletion

03

03

03

s 02.01.03 Structure identification and r

Structure identification and references

02.01 .03 .02 Change structure references

02.01 .03 .03

Change structure identifier and references

- $02.02 \quad$ Element-level Operations

$02 \quad 02.02 .01$ Opening and closing structures

$03 \quad 02.02 .02$ Miscellaneous CSS elements

0202.02 .03 Set edit mode and control of element pointer

$05 \quad 02.02 .04$ Deletion of structure elements

0102.02 .05 Copy all elements from structure

- $\quad 02.03 \quad$ Examining CSS structures

02 02.03.01 Inquiring element type and size, and content

02 s 02.03 .02 Element search

$07 \mathrm{~s} 02.03 .03$ Incremental spatial search

- 03 Archive files

0103.01 Opening and closing archive files

0103.02 Conflict resolution flags

08 s 03.03 Archiving and retrieving structures

$04 \mathrm{~s} 03.04$ Examining structures in an archive

04 s 03.05 Deleting archived structures 


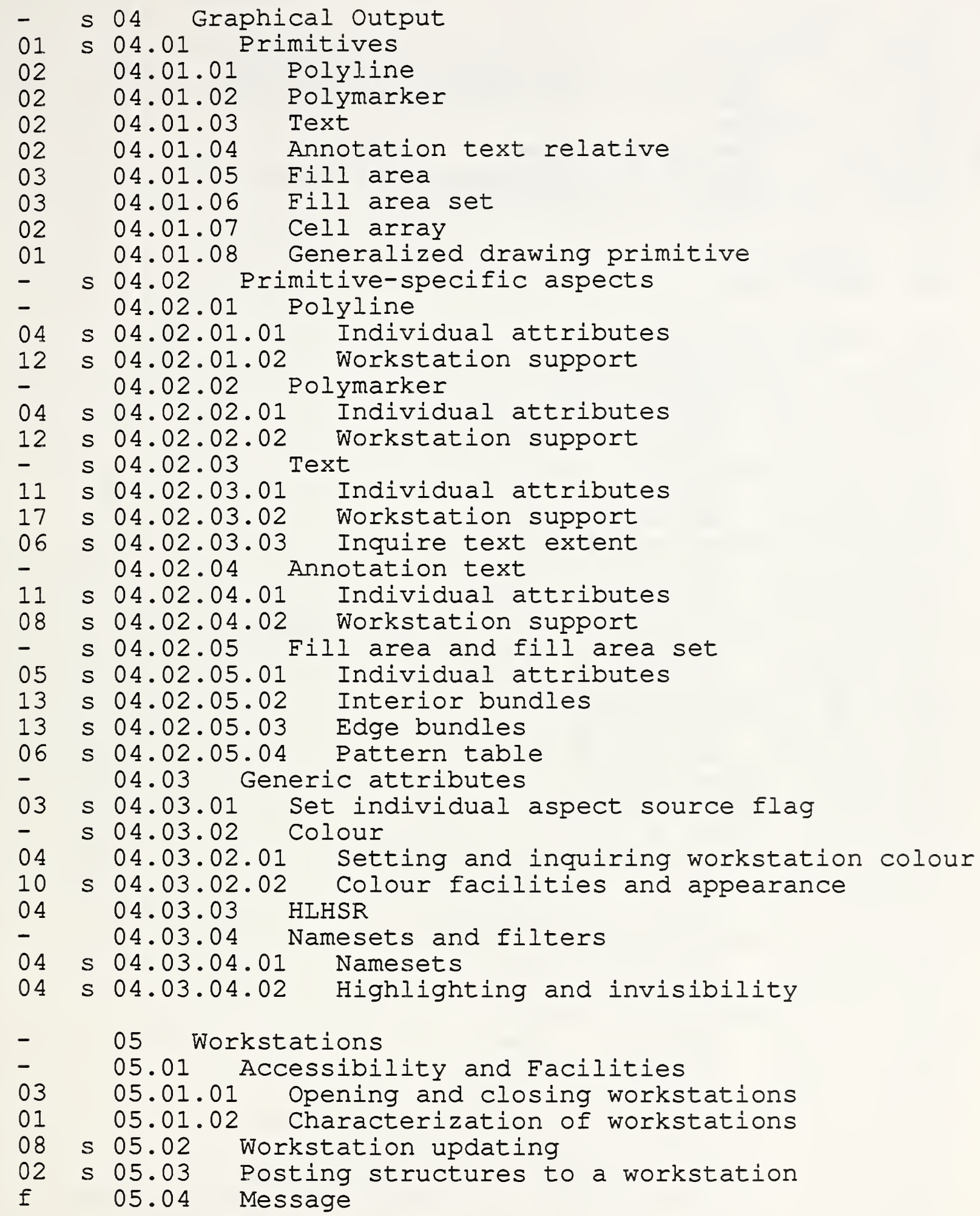


07

18

$-$

03

07

04

f

f

$-$

06

$-$

07

07

$-$

10

10

-

17

17

$-$

07

07

$-$

06

06

f

f

f

06

06

$-$

04

04

$-$

03

01

$-$

04

04

08

10

05

f

f

f

01

05

03

06 Geometry

06.01 Modelling

06.01 .01 Modelling utilities

s 06.01 .02 Modelling transformations in the CSS 06.02 Viewing

s 06.02 .01 Viewing utilities

s 06.02 .02 Control of workstation view table 06.03 Workstation transformations

\section{Input \\ 08 Metafiles}

09 Error conditions

s 09.01 Error handling

s 09.01.01 Operating state errors

09.01.01.01 Handled by user

09.01.01.02 Handled by system

s 09.01 .02 Workstation errors

09.01 .02 .01 Handled by user

09.01 .02 .02 Handled by system

s 09.01 .03 Output attribute errors

09.01 .03 .01 Handled by user

09.01 .03 .02 Handled by system

s 09.01 .04 Transformation and viewing errors

09.01.04.01 Handled by user

09.01 .04 .02 Handled by system

s 09.01.05 Structure errors

09.01 .05 .01 Handled by user

09.01 .05 .02 Handled by system

09.01 .06 Input errors

09.01 .07 Metafile errors

09.01 .08 Escape errors

s 09.01.09 Archive and retrieve errors

09.01.09.01 Handled by user

09.01.09.02 Handled by system

s 09.01 .10 Fortran-specific errors

09.01.10.01 Handled by user

09.01.10.02 Handled by system

s 09.01.11 C-specific errors

09.01.11.01 Handled by user

09.01.11.02 Handled by system

s 09.02 Reported error indicator

09.02.01 Operating state error indicators

09.02 .02 Workstation error indicators

09.02 .03 Output attribute error indicators

09.02 .04 Transformation and viewing error indicators

09.02 .05 Structure error indicators

09.02 .06 Input error indicators

09.02 .07 Metafile error indicators

09.02.08 Escape error indicators

09.02.09 Archive.retrieve error indicators

09.02.10 FORTRAN specific error indicators

09.02.11 C specific error indicators 


$\begin{array}{lll}\mathrm{f} & 10 \quad \text { Escape } \\ - & 11 \quad \text { Language-specific features } \\ 01 & 11.01 & \text { Eortran utilities } \\ 05 & 11.02 & \text { Features specific to } \mathrm{C}\end{array}$





\section{APPENDIX C \\ VERSION HISTORY OF THE PVT}

The following table summarizes the parts of the PHIGS standard tested by successive versions of the PVT. "na" indicates "not applicable", "--" indicates area not yet (if ever) covered by PVT. Coverage is the same for Fortran and $C$.

01. Opening and closing PHIGS

02. Manipulating the CSS

03. Archives

04. Graphical Output

05. Workstation Control

06 . Geometry

06.01 Modeling

06.02 Viewing

06.03 Workstation transformations

07 . Input

08. Metafiles

Passive Interactive

09. Error handling

10. Escape

11. Language-specific features

$\mathrm{v} 1.0$

v1.0

na

v1.0

na

$\mathrm{v} 1.0$

na

v1.0

$\mathrm{v} 2.0$

v1.0

v1.0

$--$

v1.0

v2. 1

$--$

$--$

$-$

$--$

na

v2.1

na

$-$

$--$

$\mathrm{v} 1.0$

na

PHIGS-PLUS 


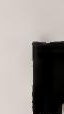

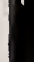

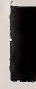

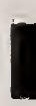

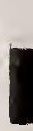

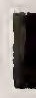

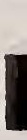

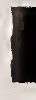

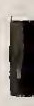

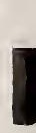

1

4

I

1

1

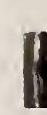

If 
APPENDIX D

TEST PROGRAM CHARACTERISTICS

The following table summarizes the CHARACTERISTICS entry for all of the PVT programs. See section 2.5.6.1 for an explanation of the codes.

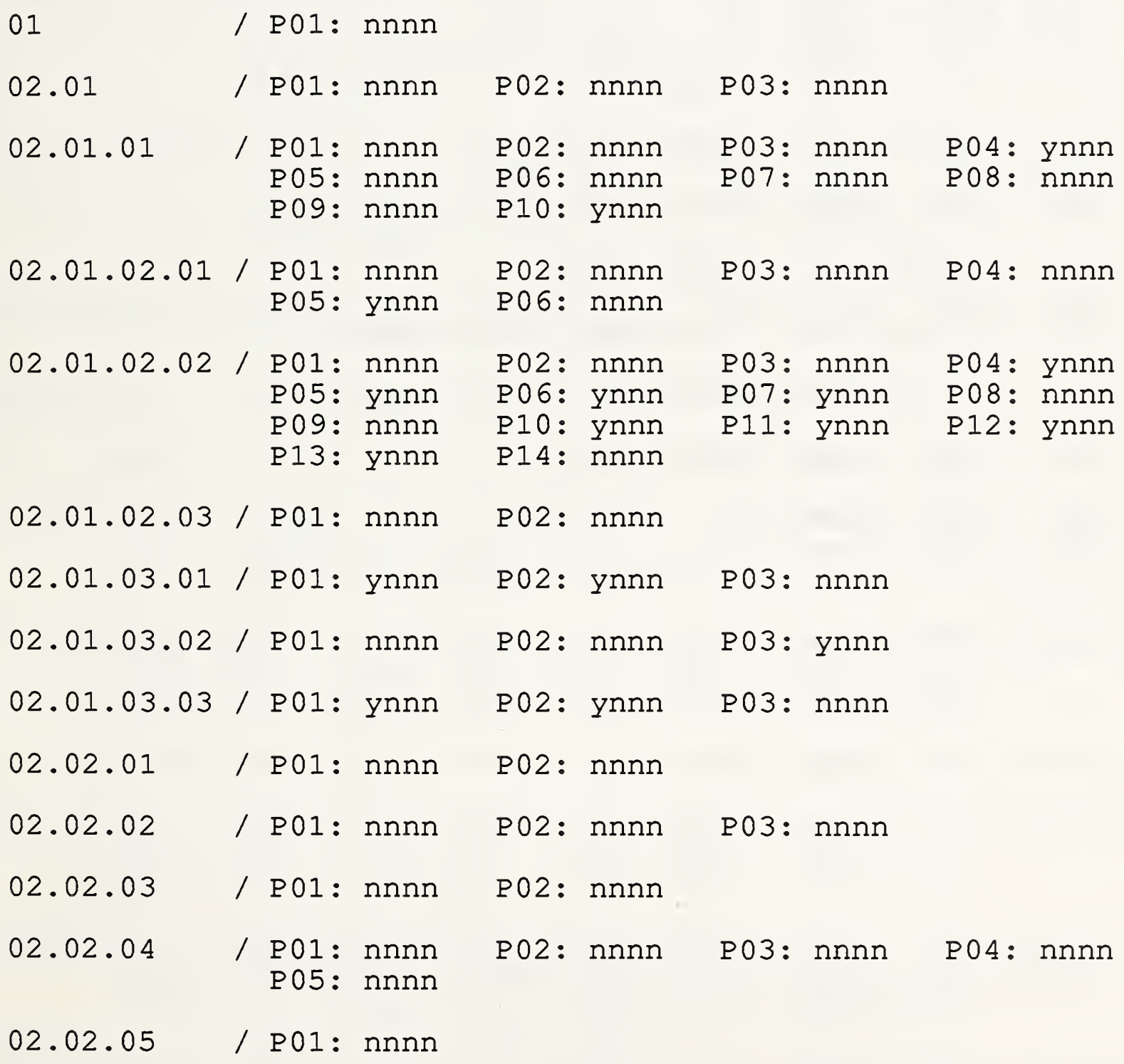




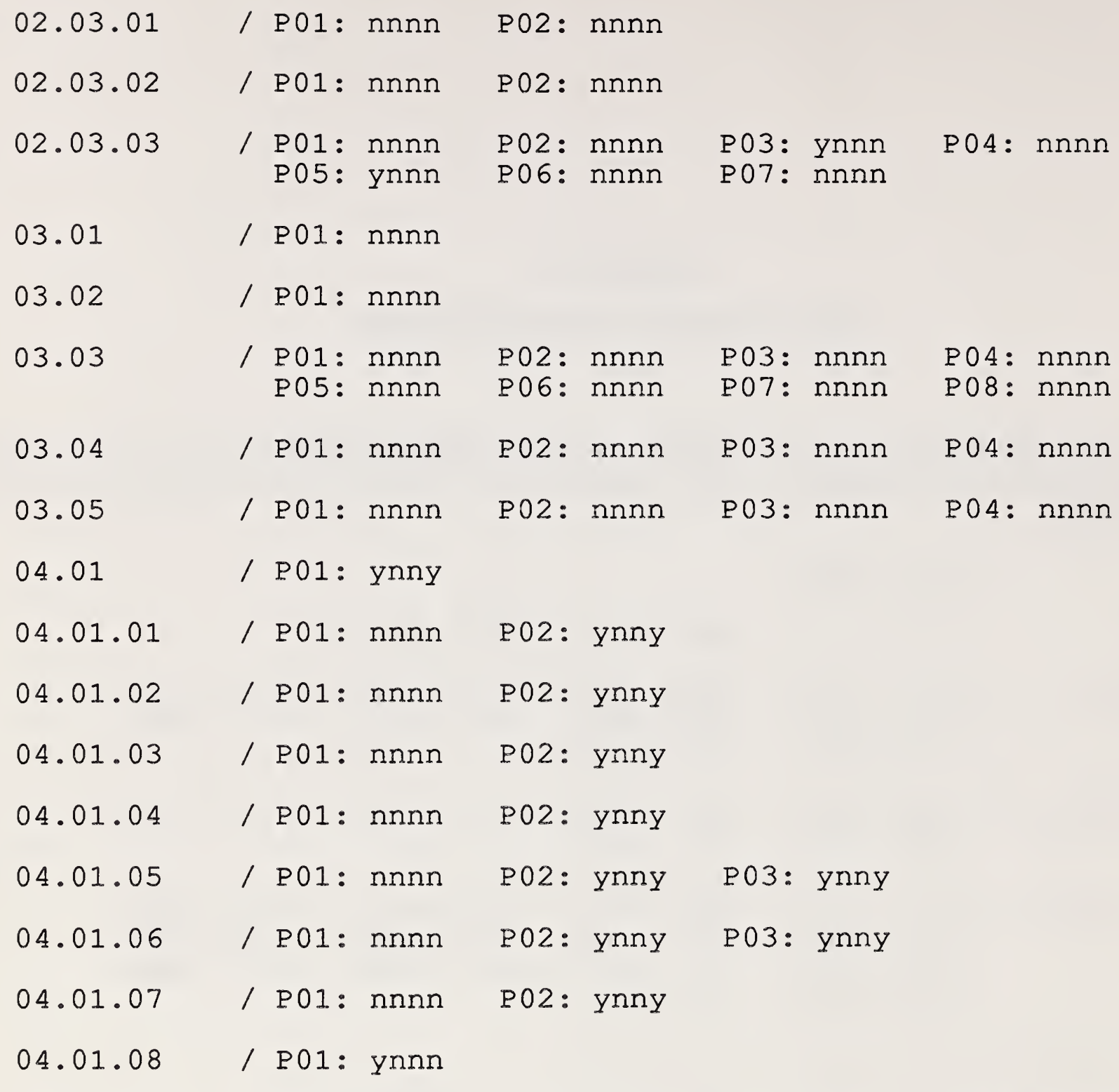

04.02.01.01/P01: nnnn P02: ynny P03: ynny P04: ynny

04.02.01.02 / P01: ynnn P02: ynnn P03: ynnn P04: ynnn P05: ynnn P06: ynnn P07: ynnn P08: ynnn P09: ynny P10: ynny P11: ynny P12: ynny

04.02.02.01/P01: nnnn P02: ynny P03: ynny P04: ynny

04.02.02.02 / P01: ynnn P02: ynnn P03: ynnn P04: ynnn P05: ynnn P06: ynnn P07: ynnn P08: ynnn P09: ynny P10: ynny P11: ynny P12: ynny

04.02.03.01 / P01: nnnn P02: ynny P03: ynny P04: ynny P05: ynny P06: ynny P07: ynny P08: ynny P09: ynny P10: ynny

P11: ynny

04.02.03.02 / P01: ynnn P02: ynnn P03: ynnn P04: ynnn P05: ynnn P06: ynnn P07: ynnn P08: ynnn 


\begin{tabular}{|c|c|c|c|c|c|c|c|c|c|}
\hline & & $\begin{array}{l}\text { P09: } \\
\text { P13: } \\
\text { P17: }\end{array}$ & $\begin{array}{l}\text { ynnn } \\
\text { ynny } \\
\text { ynny }\end{array}$ & $\begin{array}{l}\text { P10: } \\
\text { P14: }\end{array}$ & $\begin{array}{l}\text { ynny } \\
\text { ynny }\end{array}$ & $\begin{array}{l}\text { P11: } \\
\text { P15: }\end{array}$ & $\begin{array}{l}\text { ynny } \\
\text { ynny }\end{array}$ & $\begin{array}{l}\text { P12: } \\
\text { P16: }\end{array}$ & $\begin{array}{l}\text { ynny } \\
\text { ynny }\end{array}$ \\
\hline 04.02 .03 .03 & / & $\begin{array}{l}\text { P01: } \\
\text { P05: }\end{array}$ & $\begin{array}{l}\text { ynnn } \\
\text { ynnn }\end{array}$ & $\begin{array}{l}\text { P02: } \\
\text { P06: }\end{array}$ & $\begin{array}{l}\text { ynnn } \\
\text { ynny }\end{array}$ & P03: & ynnn & P0 4: & ynnn \\
\hline 04.02 .04 .01 & / & $\begin{array}{l}\text { P01: } \\
\text { P05: } \\
\text { P09: }\end{array}$ & $\begin{array}{l}\text { nnnn } \\
\text { ynny } \\
\text { ynny }\end{array}$ & $\begin{array}{l}\text { P02: } \\
\text { P06: } \\
\text { P10: }\end{array}$ & $\begin{array}{l}\text { ynny } \\
\text { ynny } \\
y n n y\end{array}$ & $\begin{array}{l}\text { P03: } \\
\text { P07: } \\
\text { P11: }\end{array}$ & $\begin{array}{l}\text { ynny } \\
\text { ynny } \\
\text { ynny }\end{array}$ & $\begin{array}{l}\text { P04: } \\
\text { P08: }\end{array}$ & $\begin{array}{l}\text { ynny } \\
\text { ynny }\end{array}$ \\
\hline 04.02 .04 .02 & / & $\begin{array}{l}\text { P01: } \\
\text { P05: }\end{array}$ & $\begin{array}{l}\text { ynny } \\
\text { ynny }\end{array}$ & $\begin{array}{l}\text { P02: } \\
\text { P06: }\end{array}$ & $\begin{array}{l}\text { ynny } \\
\text { ynny }\end{array}$ & $\begin{array}{l}\text { P03: } \\
\text { P07: }\end{array}$ & $\begin{array}{l}\text { ynny } \\
\text { ynny }\end{array}$ & $\begin{array}{l}\text { P04: } \\
\text { P08: }\end{array}$ & $\begin{array}{l}\text { ynny } \\
\text { ynny }\end{array}$ \\
\hline 04.02 .05 .01 & / & $\begin{array}{l}\text { P01: } \\
\text { P05: }\end{array}$ & $\begin{array}{l}\text { nnnn } \\
\text { ynny }\end{array}$ & P02: & ynny & P03: & ynny & P04: & ynny \\
\hline 04.02 .05 .02 & / & $\begin{array}{l}\text { P01: } \\
\text { P05: } \\
\text { P09: } \\
\text { P13: }\end{array}$ & $\begin{array}{l}\text { ynnn } \\
\text { ynnn } \\
\text { ynnn } \\
\text { ynny }\end{array}$ & $\begin{array}{l}\text { P02: } \\
\text { P06: } \\
\text { P10: }\end{array}$ & $\begin{array}{l}\text { ynnn } \\
\text { ynnn } \\
\text { ynny }\end{array}$ & $\begin{array}{l}\text { P03: } \\
\text { P07: } \\
\text { P11: }\end{array}$ & $\begin{array}{l}\text { ynnn } \\
\text { ynnn } \\
\text { ynny }\end{array}$ & $\begin{array}{l}\text { P04: } \\
\text { P08: } \\
\text { P12: }\end{array}$ & $\begin{array}{l}\text { ynnn } \\
\text { ynnn } \\
\text { ynny }\end{array}$ \\
\hline 04.02 .05 .03 & 1 & $\begin{array}{l}\text { P01: } \\
\text { P05: } \\
\text { P09: } \\
\text { P13: }\end{array}$ & $\begin{array}{l}\text { ynnn } \\
\text { ynnn } \\
\text { ynnn } \\
\text { ynny }\end{array}$ & $\begin{array}{l}\text { P02: } \\
\text { P06: } \\
\text { P10: }\end{array}$ & $\begin{array}{l}\text { ynnn } \\
\text { ynnn } \\
\text { ynny }\end{array}$ & $\begin{array}{l}\text { P03: } \\
\text { P07: } \\
\text { P11: }\end{array}$ & $\begin{array}{l}\text { ynnn } \\
\text { ynnn } \\
\text { ynny }\end{array}$ & $\begin{array}{l}\text { P04: } \\
\text { P08: } \\
\text { P12: }\end{array}$ & $\begin{array}{l}\text { ynnn } \\
\text { ynnn } \\
\text { ynny }\end{array}$ \\
\hline 04.02 .05 .04 & 1 & $\begin{array}{l}\text { P01: } \\
\text { P05: }\end{array}$ & $\begin{array}{l}\text { ynnn } \\
\text { ynnn }\end{array}$ & $\begin{array}{l}\text { P02: } \\
\text { P06: }\end{array}$ & $\begin{array}{l}\text { ynnn } \\
\text { ynnn }\end{array}$ & P03: & ynnn & P04: & ynnn \\
\hline 04.03 .01 & / & P01: & nnnn & P02: & ynny & P03: & ynny & & \\
\hline 04.03 .02 .01 & / & P01: & ynnn & P02: & ynnn & P03: & ynnn & P04: & ynny \\
\hline 04.03 .02 .02 & / & $\begin{array}{l}\text { P01: } \\
\text { P05: } \\
\text { P09: }\end{array}$ & $\begin{array}{l}y n n n \\
y n n n \\
y n n y\end{array}$ & $\begin{array}{l}\text { P02: } \\
\text { P06: } \\
\text { P10: }\end{array}$ & $\begin{array}{l}\text { ynnn } \\
\text { ynny } \\
\text { ynny }\end{array}$ & $\begin{array}{l}\text { P03: } \\
\text { P07: }\end{array}$ & $\begin{array}{l}\text { ynnn } \\
\text { ynny }\end{array}$ & $\begin{array}{l}\text { P04: } \\
\text { P08: }\end{array}$ & $\begin{array}{l}\text { ynnn } \\
\text { ynny }\end{array}$ \\
\hline 04.03 .03 & / & P01: & ynnn & P02: & ynnn & P03: & ynnn & P04: & ynnn \\
\hline 04.03 .04 .01 & / & P01: & nnnn & P02: & nnnn & P03: & nnnn & P04: & ynny \\
\hline 04.03 .04 .02 & / & P01: & ynnn & P02: & ynnn & P03: & ynny & P04: & ynny \\
\hline 05.01 .01 & / & P01: & nnnn & P02: & nnnn & P03: & nnnn & & \\
\hline 05.01 .02 & / & P01: & nnnn & & & & & & \\
\hline 05.02 & / & $\begin{array}{l}\text { P01: } \\
\text { P05: }\end{array}$ & $\begin{array}{l}\text { ynnn } \\
\text { ynnn }\end{array}$ & $\begin{array}{l}\text { P02: } \\
\text { P06: }\end{array}$ & $\begin{array}{l}\text { ynnn } \\
\text { yynn }\end{array}$ & $\begin{array}{l}\text { P03: } \\
\text { P07: }\end{array}$ & $\begin{array}{l}\text { ynnn } \\
\text { ynnn }\end{array}$ & $\begin{array}{l}\text { P04: } \\
\text { P08: }\end{array}$ & $\begin{array}{l}\text { ynnn } \\
\text { ynnn }\end{array}$ \\
\hline 05.03 & / & P01: & ynnn & P02: & ynnn & & & & \\
\hline 06.01 .01 & / & P01: & nnnn & P02: & nnnn & P03: & nnnn & P04: & nnnn \\
\hline
\end{tabular}




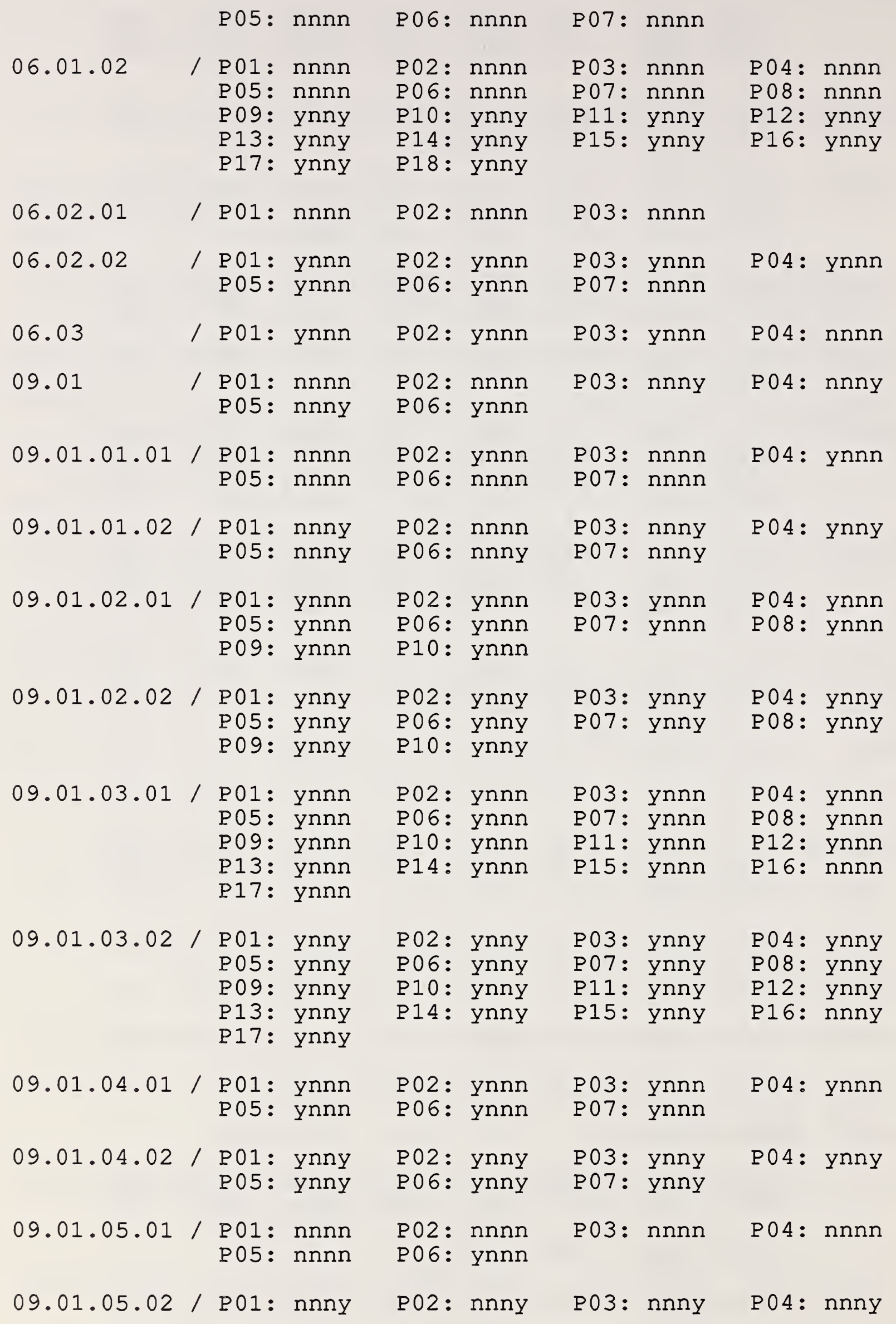


P05: nnny P06: ynny

$\begin{aligned} 09.01 .09 .01 / \text { P01: nnnn P02: nnnn P03: nnnn P04: nnnn } & \\ \text { P05: nnnn P06: nnnn } & \end{aligned}$ 09.01.09.02 / P01: nnny P02: nnny P03: nnny P04: nnny P05: nnny P06: nnny

09.01.10.01 / P01: ynnn P02: nnnn P03: ynnn P04: nnnn 09.01.10.02 / P01: ynny P02: nnny P03: ynny P04: nnny 09.01.11.01/P01: nnnn P02: nnnn P03: ynnn 09.01 .11 .02 / P01: ynny 09.02.01 / P01: nnnn P02: nnnn P03: nnnn P04: nnnn 09.02.02 / P01: nnnn P02: ynnn P03: ynnn P04: ynnn 09.02.03 / P01: ynnn P02: ynnn P03: ynnn P04: ynnn P05: ynnn P06: ynnn P07: ynnn P08: ynnn

09.02.04 / P01: nnnn P02: nnnn P03: nnnn P04: nnnn P05: nnnn P06: nnnn P07: nnnn P08: nnnn P09: nnnn P10: nnnn

09.02.05 / P01: ynnn P02: nnnn P03: nnnn P04: nnnn P05: nnnn

09.02 .09 / P01: nnnn

09.02.10 / P01: ynnn P02: ynnn P03: ynnn P04: ynnn

09.02.11 / P01: ynnn P02: ynnn P03: ynnn

$11.01 / \mathrm{P} 01: \mathrm{nnnn}$

11.02 / P01: nnnn P02: nnnn P03: nnnn P04: nnnn P05: nnnn 


\section{APPENDIX E \\ DICTIONARY OE SUBROUTINES AND FUNCTIONS}

This table lists all subroutines and functions in the PVT, in alphabetical order. The two columns following each function name indicate whether it is a subroutine or function, and its location. For example, ACTST is a subroutine in pvt 02 01 03 sublib.f, APPEQ is a logical function in pvt sublib.f, and ARRTP3 is a subroutine in pvt trans-sublib.f.

\begin{tabular}{|c|c|c|}
\hline bend & SUBROUT & GL \\
\hline actst & SUBROUT & 02.01 .03 \\
\hline ppeq & LOGC-EN & GL \\
\hline rcpts & SUBROUT & 04 \\
\hline $\operatorname{rrtp} 3$ & SUBROUT & TR \\
\hline sfset & SUBROUT & 04.03 .01 \\
\hline asbot & REAL-EN & 04.02 \\
\hline 01002 & SUBROUT & 09.01 .01 \\
\hline 01004 & SUBROUT & 09.01 .01 \\
\hline 01006 & SUBROUT & 09.01 .01 \\
\hline eilng & INTG-EN & TR \\
\hline chkfnt & SUBROUT & 04.02 \\
\hline hkmev & SUBROUT & 06.01 .02 \\
\hline hmono & SUBROUT & 03.03 \\
\hline cldiag & SUBROUT & 04.02 \\
\hline clpgon & SUBROUT & 06.01 .02 \\
\hline coleql & LOGC-EN & 04.03 .02 .02 \\
\hline colpch & SUBROUT & 04.03 .02 .02 \\
\hline comtok & SUBROUT & 06.01 .02 \\
\hline crest & SUBROUT & 09.01 \\
\hline css & LOGC-EN & GL \\
\hline th & SUBROUT & GL \\
\hline dchfl & LOGC-EN & GL \\
\hline dchoic & SUBROUT & GL \\
\hline fv & OUT & GL \\
\hline ss & SUI & 02.01 .02 .02 \\
\hline st & SUB & GL \\
\hline$d b$ & SUB & 04.02 .0 \\
\hline $1 b$ & SUB & 04.02 .01 .0 \\
\hline & SUBF & 04.02 \\
\hline & SUB & \\
\hline & & \\
\hline
\end{tabular}

\begin{tabular}{|c|c|c|}
\hline & INTG-FN & TR \\
\hline siz & SUBROUT & 04.02 \\
\hline cpth & SUBROUT & 03.04 \\
\hline req & LOGC-EN & 04.02 .05 .0 \\
\hline fint & SUBROUT & 04 \\
\hline arnm & SUBROUT & GL \\
\hline nsg & SUBROUT & GL \\
\hline & SUBROUT & 09.01 .01 \\
\hline 5 & SUBROUT & 09.01 \\
\hline 7 & SUBROUT & 09.01 .01 \\
\hline lp & SUB & GL \\
\hline inq & SUB & GL \\
\hline pst & SUB & 05.03 \\
\hline $\operatorname{xrc}$ & SUB & 04.02 .03 .0 \\
\hline ir & SUB & 06.01 .02 \\
\hline omk 5 & SUB & 06.01 .02 \\
\hline nam & SUB. & 04.03 .02 \\
\hline $\operatorname{trn}$ & SUB. & 04.03 .02 .0 \\
\hline iar & SUBI & GL \\
\hline 0 & SUBROUT & TR \\
\hline ds & LOGC-FN & 03.03 \\
\hline & SUBROUT & 04.03 .04 .0 \\
\hline IV & LOGC-EN & GL \\
\hline$p$ & SUE & GL \\
\hline$n k$ & SUE & GL \\
\hline & SUI & GL \\
\hline & SUI & 04 \\
\hline & SUE & 04.02 .05 .02 \\
\hline & SUI & 04.02 .02 .0 \\
\hline & SUE & GL \\
\hline & SUE & 05. \\
\hline & & 01 \\
\hline
\end{tabular}


drIval SUBROUT GL

drwedb SUBROUT 04.02.05.03

drwplb SUBROUT 04.02.01.02

drwrec SUBROUT GL

ds 3

dstrng

dynpf

e01003

e 01005

e01007

e 02053

e 02056

e 02058

e 02060

e02063

e03101

e03104

e03106

e03108

e03111

e03113

e03115

e03117

e 04150

e 04152

e 04154

e 04157

e05201

e 05206

e 05208

e 09402

e 09405

e 09408

e12001

e12005

ebltm

echkz

ecom 3

ecotm3

eevom

elgprm

enderr

erfucd

ermsgh

erox

eroz

esc

esetup

etp 3

etr 3

exp34

expppm

faclip

filrec

SUBROUT 09.01.04

SUBROUT GL

SUBROUT GL

SUBROUT 09.01.01

SUBROUT 09.01.01

SUBROUT 09.01.01

SUBROUT 09.01.02

SUBROUT 09.01.02

SUBROUT 09.01.02

SUBROUT 09.01.02

SUBROUT 09.01.02

SUBROUT 09.01.03

SUBROUT 09.01.03

SUBROUT 09.01.03

SUBROUT 09.01.03

SUBROUT 09.01.03

SUBROUT 09.01.03

SUBROUT 09.01.03

SUBROUT 09.01.03

SUBROUT 09.01.04

SUBROUT 09.01.04

SUBROUT 09.01.04

SUBROUT 09.01.04

SUBROUT 09.01.05

SUBROUT 09.01.05

SUBROUT 09.01.05

SUBROUT 09.01.09

SUBROUT 09.01.09

SUBROUT 09.01.09

SUBROUT 09.01.10

SUBROUT 09.01.10

SUBROUT TR

SUBROUT 09.01

SUBROUT TR

SUBROUT TR

SUBROUT TR

SUBROUT 04.03 .04 .02

SUBROUT 09.01

SUBROUT GI

LOGC-FN 09.01

SUBROUT TR

SUBROUT TR

SUBROUT TR

SUBROUT 09.01

SUBROUT TR

SUBROUT TR

SUBROUT TR

SUBROUT 04.02 .02 .01

SUBROUT 06.01 .02

SUBROUT 04.01 drwatx

drwint

drwpmb

drwtxt

dsanst

dyn

e01001

e 01004

e 01006

e 02052

e 02054

e 02057

e 02059

e 02061

e 03100

e 03103

e 03105

e03107

e03110

e 03112

e 03114

e 03116

e 03118

e 04151

e 04153

e 04156

e 05200

e 05205

e 05207

e 09401

e 09404

e 09407

e12000

e 12004

e 12202

ebltm3

ecom

ecotm

eevmm

eevom3

elstr

endit

erfunm

ero

eroy

errct 1

esc3

etp

etr

exedv

expppl

exsvrs

fail

fitpar
SUBROUT 04.02 .04 .02

SUBROUT 04.02 .05 .02

SUBROUT 04.02 .02 .02

SUBROUT 04.02 .03 .02

SUBROUT 04.02 .04 .01

LOGC-FN GL

SUBROUT 09.01.01

SUBROUT 09.01.01

SUBROUT 09.01.01

SUBROUT 09.01 .02

SUBROUT 09.01.02

SUBROUT 09.01.02

SUBROUT 09.01.02

SUBROUT 09.01.02

SUBROUT 09.01.03

SUBROUT 09.01.03

SUBROUT 09.01.03

SUBROUT 09.01 .03

SUBROUT 09.01.03

SUBROUT 09.01.03

SUBROUT 09.01.03

SUBROUT 09.01.03

SUBROUT 09.01 .03

SUBROUT 09.01.04

SUBROUT 09.01.04

SUBROUT 09.01.04

SUBROUT 09.01.05

SUBROUT 09.01 .05

SUBROUT 09.01.05

SUBROUT 09.01.09

SUBROUT 09.01.09

SUBROUT 09.01.09

SUBROUT 09.01.10

SUBROUT 09.01.10

SUBROUT 09.01.11

SUBROUT TR

SUBROUT TR

SUBROUT TR

SUBROUT TR

SUBROUT TR

SUBROUT 02.01 .03

SUBROUT GL

SUBROUT GI

SUBROUT TR

SUBROUT TR

SUBROUT GL

SUBROUT TR

SUBROUT TR

SUBROUT TR

SUBROUT 06.01 .02

SUBROUT 04.02 .01 .01

SUBROUT 05.02

SUBROUT GL

SUBROUT GL 


\begin{tabular}{|c|c|c|}
\hline fltran & SUBROUT & 02.03 .03 \\
\hline geotxt & SUBROUT & 04.02 .03 \\
\hline gtroot & SUBROUT & GL \\
\hline hueang & SUBROUT & 04.03 .02 .02 \\
\hline iacmsg & SUBROUT & GL \\
\hline iareql & LOGC-FN & GL \\
\hline ichkz & SUBROUT & 09.02 \\
\hline ifpf & SUBROUT & GI \\
\hline ifuncs & SUBROUT & 09.02 \\
\hline iniari & SUBROUT & GL \\
\hline inmsg & SUBROUT & GL \\
\hline intinh & SUBROUT & 04.03 .01 \\
\hline invol & SUBROUT & TR \\
\hline issab & SUBROUT & TR \\
\hline issflt & SUBROUT & 02.03 .03 \\
\hline isspf & SUBROUT & 02.03 .03 \\
\hline Ibedty & SUBROUT & 04.02 .05 .03 \\
\hline linbrk & SUBROUT & GL \\
\hline lnplpt & SUBROUT & TR \\
\hline locat 2 & SUBROUT & 04.02 .04 .01 \\
\hline locint & SUBROUT & 04 \\
\hline locppl & SUBROUT & 04 \\
\hline locrec & SUBROUT & 04.02 .05 \\
\hline $10 c t \times 1$ & SUBROUT & 04.02 .03 .01 \\
\hline loctxt & SUBROUT & 04 \\
\hline $\bmod 1$ & INTG-FN & TR \\
\hline modtrn & INTG-FN & 06.01 .02 \\
\hline ncmsg & SUBROUT & GL \\
\hline ndmw & SUBROUT & 04.02 .02 .01 \\
\hline nfunm & SUBROUT & GL \\
\hline nmscol & SUBROU'T & 04.03 .04 .02 \\
\hline numlab & SUBROUT & GL \\
\hline opcofl & SUBROUT & GL \\
\hline pfail & SUBROUT & GL \\
\hline plin & SUBROUT & GL \\
\hline omsgw & SUBROUT & GL \\
\hline $\operatorname{arcof}$ & SUBROUT & TR \\
\hline arpt & SUBROUT & 04.02 .05 .01 \\
\hline atavl & LOGC-EN & 04.02 .05 .04 \\
\hline erhnd & SUBROUT & $\mathrm{ER}$ \\
\hline & SUBROUT & GI \\
\hline icenv & SUBROUT & 05.02 \\
\hline $2 \mathrm{pl}$ & SUBROUT & $T R$ \\
\hline nattr & SUBROUT & 04.02 .02 .01 \\
\hline p $p 1$ & SUBROUT & TR \\
\hline esrl & SUBROUT & GL \\
\hline stctl & SUBROUT & GL \\
\hline $3 p l$ & SUBROUT & TR \\
\hline Inds & SUBROUT & TR \\
\hline regd & REAL-FN & TR \\
\hline nary & SUBROUT & 06.01 .02 \\
\hline nmkt & INTG-EN & 04.02 .02 .01 \\
\hline d43 & SUBROUT & TR \\
\hline unc & SUBROUT & 09.0 \\
\hline
\end{tabular}

\begin{tabular}{|c|c|c|}
\hline fpavl & LOGC-EN & 04.02 \\
\hline gterrm & SUBROUT & 09.01 \\
\hline hlfbox & SUBROUT & 04.02 \\
\hline iacans & SUBROUT & GL \\
\hline iareq & LOGC-EN & GL \\
\hline iarfind & INTG-EN & GL \\
\hline idmat & SUBROUT & TR \\
\hline ifphcl & SUBROUT & 09.01 .01 \\
\hline inarea & INTG-EN & TR \\
\hline initgl & SUBROUT & GL \\
\hline inoutl & SUBROUT & 04.02 .03 \\
\hline intsty & LOGC-EN & GL \\
\hline ispths & SUBROUT & $T R$ \\
\hline issdis & SUBROUT & TR \\
\hline issgeo & SUBROUT & 02.03 .03 \\
\hline itrim & INTG-FN & GL \\
\hline leadnb & INTG-FN & GL \\
\hline lintpt & SUBROUT & TR \\
\hline locans & SUBROUT & 04.02 .04 .01 \\
\hline locat $x$ & SUBROUT & 04 \\
\hline $10 \mathrm{cnpm}$ & SUBROUT & 06.01 .02 \\
\hline locppm & SUBROUT & GL \\
\hline loctri & SUBROUT & 04 \\
\hline $10 \mathrm{ct} \times 2$ & SUBROUT & 04.02 .03 .01 \\
\hline matmul & SUBROUT & TR \\
\hline modsep & REAL-FN & 04.03 .02 .02 \\
\hline multws & SUBROUT & GL \\
\hline ndlw & SUBROUT & 04.02 .01 .01 \\
\hline newstr & SUBROUT & GL \\
\hline ngtxat & SUBROUT & 04.02 \\
\hline nmsprm & SUBROUT & 04.03 .04 .02 \\
\hline opco & SUBROUT & GL \\
\hline opcomt & SUBROUT & GL \\
\hline opint & SUBROUT & GL \\
\hline opmsg & SUBROUT & GL \\
\hline opyn & SUBROUT & GL \\
\hline parlin & SUBROUT & 04.02 .05 .01 \\
\hline pass & SUBROUT & GL \\
\hline patrec & SUBROUT & 04.02 .05 \\
\hline pervec & SUBROUT & TR \\
\hline picchg & SUBROUT & 05.02 \\
\hline pl2ln & SUBROUT & TR \\
\hline plattr & SUBROUT & 04.02 .01 .01 \\
\hline prpfint & SUBROUT & 04.02 \\
\hline prsint & SUBROUT & GI \\
\hline prsuin & SUBROUT & GL \\
\hline pt 2 cof & SUBROUT & $\mathrm{TR}$ \\
\hline pthseq & SUBROUT & 02.01 \\
\hline ptplds & SUBROUT & TR \\
\hline ptspl & SUBROUT & TR \\
\hline ranlst & INTG-FN & 04.02 .01 .01 \\
\hline rareq & LOGC-FN & GL \\
\hline retopn & SUBROUT & 03.01 \\
\hline rlzew & LOGC-FN & 04.02 .05 .03 \\
\hline
\end{tabular}




\begin{tabular}{|c|c|c|}
\hline$=l z l \mathrm{w}$ & LOGC-EN & 04 \\
\hline rnlshf & SUBROUT & GL \\
\hline nd01 & REAL-EN & GL \\
\hline $2 d r l$ & REAL-FN & GL \\
\hline iset & SUBROUT & GL \\
\hline capar & SUBROUT & GL \\
\hline $\operatorname{et} 2 d$ & SUBROUT & GL \\
\hline tdif & SUBROUT & 03.05 \\
\hline teq & LOGC-FN & GI \\
\hline thue & SUBROUT & 04.03 .02 .02 \\
\hline tmsg & SUBROUT & GL \\
\hline tpst & SUBROUT & 05.03 \\
\hline etstr & SUBROUT & GL \\
\hline tval & SUBROUT & GL \\
\hline edbw & SUBROUT & 04.02 .05 .03 \\
\hline OWlW & SUBROUT & \\
\hline plbw & SUBROUT & 04.02 .0 \\
\hline gmsg & SUBROUT & GL \\
\hline rk & SUBROUT & 04.02 \\
\hline grmk & SUBROUT & 04.03 .02 \\
\hline Erar & SUBROUT & GL \\
\hline rcon & LOGC-FN & GL \\
\hline bfnt & SUBROUT & 04.02 \\
\hline rok & LOGC-FN & 05.02 \\
\hline scir & SUBROUT & 02.01 .03 \\
\hline nseq & LOGC-FN & $\mathrm{TR}$ \\
\hline chup & SUBROUT & 04.02 .03 \\
\hline tcid & SUBROUT & 02.01 .03 \\
\hline $\operatorname{tcor}$ & SUBROUT & 06.02 .01 \\
\hline terr & SUBROUT & 09.01 \\
\hline thue & INTG-FN & 04.03 .02 .02 \\
\hline tivf & SUBROUT & 04.03 .04 .0 \\
\hline Evip & SUBROUT & 06.0 \\
\hline 2 dex & SUBROUT & 02.03 .03 \\
\hline al & SUBROUT & GL \\
\hline and & LOGC-FN & 04.03 .02 .02 \\
\hline & SUBROUT & 04.02 \\
\hline & SUBROUT & $\mathrm{TR}$ \\
\hline & REAL-FN & $\mathrm{TR}$ \\
\hline$\$ \mathrm{ab}$ & SUBROUT & GL \\
\hline & SUBROUT & GL \\
\hline & SUBROUT & 04 \\
\hline-5 & REAL-FN & GL \\
\hline & T ד & 02 \\
\hline
\end{tabular}

\begin{tabular}{|c|c|c|}
\hline rlzms & LOGC-FN & 04.02 .02 .02 \\
\hline rnbset & SUBROUT & GL \\
\hline rndint & INTG-EN & GL \\
\hline rnperm & SUBROUT & GL \\
\hline satan2 & REAL-EN & TR \\
\hline scherr & SUBROUT & 09.01 \\
\hline setasf & SUBROUT & GL \\
\hline setdlg & SUBROUT & GL \\
\hline setfil & SUBROUT & 02.03 .03 \\
\hline setis & LOGC-FN & GL \\
\hline setpcl & SUBROUT & 05.02 \\
\hline setrvs & SUBROUT & GL \\
\hline setsvr & SUBROUT & 05.02 \\
\hline setvs & SUBROUT & GL \\
\hline showew & SUBROUT & 04.02 .05 .01 \\
\hline showmw & SUBROUT & 04.02 .02 .01 \\
\hline shpmbw & SUBROUT & 04.02 .02 .02 \\
\hline sigtst & SUBROUT & GL \\
\hline sphdis & LOGC-FN & 02.03 .03 \\
\hline srtiar & SUBROUT & GL \\
\hline stdess & SUBROUT & GL \\
\hline streq & LOGC-FN & 09.01 \\
\hline surfok & LOGC-FN & 05.02 \\
\hline tcscid & SUBROUT & 02.01 .03 \\
\hline tranhs & SUBROUT & 06.01 .02 \\
\hline tsanup & SUBROUT & 04.02 .04 .01 \\
\hline tstasc & SUBROUT & 04.02 \\
\hline tstcir & SUBROUT & 02.01 .03 \\
\hline tstels & SUBROUT & 02.03 .02 \\
\hline tsthlf & SUBROUT & 04.03 .04 .02 \\
\hline tstign & SUBROUT & 09.01 \\
\hline tstprj & SUBROUT & 06.02 .01 \\
\hline tstwan & SUBROUT & 09.01 \\
\hline txcomp & SUBROUT & 04.02 .03 .03 \\
\hline txpcup & SUBROUT & 04.02 \\
\hline unmsg & SUBROUT & GL \\
\hline varfnt & SUBROUT & 04.02 \\
\hline vecang & REAL-FN & TR \\
\hline vecl2 & REAL-FN & TR \\
\hline wenpc & SUBROUT & GL \\
\hline indup & SUBROUT & GL \\
\hline xpopph & SUBROUT & GL \\
\hline ypos 8 & REAL-FN & 04.03 .04 \\
\hline
\end{tabular}


APPENDIX E

EXAMPLES OE COMMAND PROCEDURES

Below are two examples of a compile, link, and execute procedure for PVT programs. The first is for the VAX/VMS system, the second for UNIX. These are for illustrative purposes only.

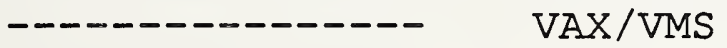

Assume the PVT root node is [USER.PVT.V2]. In order to invoke the following, the user types:

\$ @PHF PO1 ! run P01, with PVT-defined error handling or

\$ @PHEE P01 ! run P01, with implementor-defined error handling

PHF. COM:

$\$$ ! Procedure to invoke DecPHIGS and access PVT libraries.

$\$$ ! Value of variable 'errlib' is set to cause invocation

$\$$ ! of user error handler

$\$$ errlib :== "[user.pvt.v2] errlib/lib, "

$\$$ psource $=p 1$

$\$$ ephfcom

\section{PHEE.COM}

\$! Procedure to invoke DecPHIGS and access PVT libraries.

$\$$ ! Value of variable 'errlib' is set to prevent invocation

$\$$ ! of system default error handler

$\$$ errlib :==" "

$\$$ psource $=p 1$

$\$$ ephfcom

\section{PHFCOM.COM}

$\$$ ! Procedure to invoke DecPHIGS and access PVT libraries.

$\$$ ! Value of variable 'errlib' controls whether user error

$\$$ ! handling is invoked. Name of program is passed down

$\$$ ! in variable 'psource'.

$\$$ set nover

\$ define sys\$input sys\$command

$$
\mathrm{F}-1
$$


$\$$ locsub $="$ " $\quad$ this'll be string with names of local sublibs

$\$$ curdir $=$ f\$directory () ! name of current directory

$\$$ dirlen $=$ f\$length (curdir) $!$ length of current directory name

\$ stdloc = f\$locate (".v2", curdir)! get root level

$\$$ rootnam = "V2"

$\$$ if (staloc.lt. dirlen) then goto rootset

\$ write sys\$output "Cannot find V2 in current directory."

$\$$ goto alldone

$\$$ rootset:

$\$$ numlev $=($ dirlen - stdloc -4$) / 3$ ! number of levels of PVT

$\$$ backup $=0$

$\$$ !

$\$$ sub_loop:

$\$$ if Tbackup.ge. numlev) then goto end sub loop

$\$$ seekfile = "[" + f\$extract $(1$, backup, "=-------" $)+"]$ sublib"

$\$$ gotfile $=$ f\$search (seekfile $+" . o l b ") !$ blank if no sublib at this

\$ if (gotfile .nes. " ") then $\$$ locsub = locsub + seekfile + "/lib,"

$\$$ backup = backup +1

$\$$ goto sub_loop

$\$$ !

$\$$ end_sub_loop:

$\$$ set ver

$\$$ fort/warn=all/standard/float=ieee 'psource'

\$ link 'psource',' locsub' [user.pvt.' rootnam']trans_sublib/lib, [user.pvt.' rootnam'] sublib/lib, 'errlib' -

sys\$library:phigs\$for_bnd/lib

$\$$ run 'psource'

$\$$ alldone: 
Assume the PVT root node is/home/user/pvt/v2. In order to invoke th following, the user types:

\$ pvt p01 ! run p01, with PVT-defined error handling

or

\$ pvt p01 e ! run P01, with implementor-defined error handling

pvt :

\#/usr/bin/csh

\# This script:

\# Determines the language of the input file (FTN or C)

\# Compiles the source to object code

If any step fails, the script will exit before proceeding.

\# Test searching of libraries by invoking with an argument

\# of "hi". See usage at bottom for usage instructions.

Set PRE PHIGS LIB to point to your Phigs libraries. This is

\# the first library listed in the link command.

set PRE PHIGS LIB = " "

set POST PHIGS LIB to your phigs library. This is listed

\# LAST in the lin̄k command.

set POST_PHIGS_LIB $="-$ L/usr/phigs-2.0/lib -lphigs77 -lphigs -lxgl -

if ( $\$ \#$ argv $<1$ ) then

echo "Found < 1 argument"

endif

goto usage

if ( $\$$ \#argv > 2 ) then

echo "Found $>2$ arguments"

endif goto usage

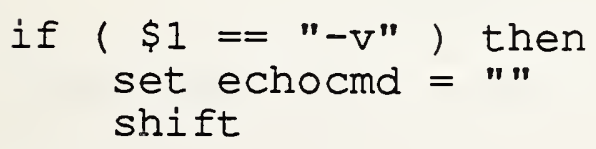

\# USER CONFIGURATION VARIABLES

\# Change libext if libraries have different extensions. set PVTROOT ='/home/user/pvt/v2' 


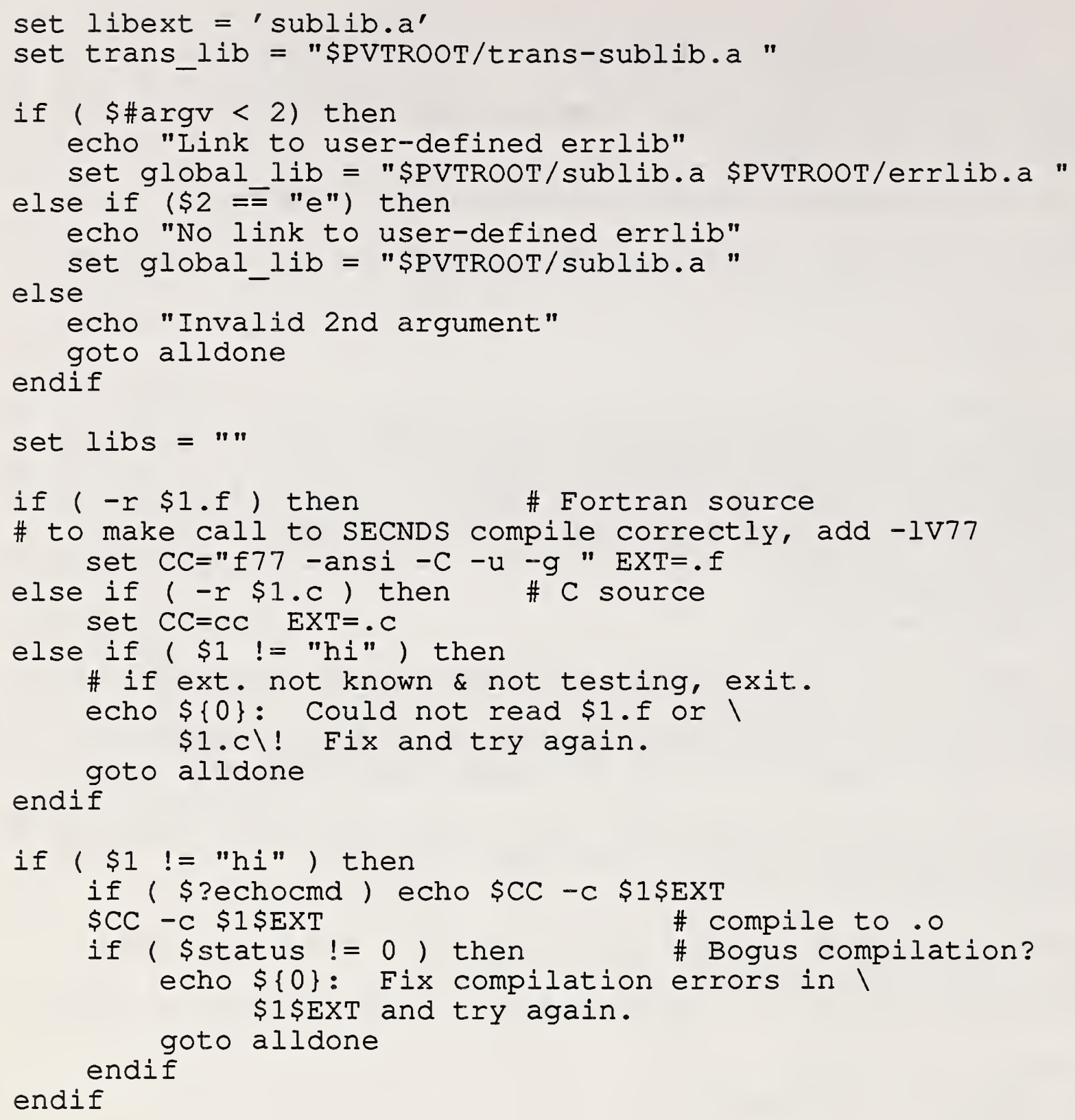

if ( $\$$ eechocmd) echo $-\mathrm{n} \$\{0\}$ : Searching for library archives...

set curdir = 'pwd'

while ( curdir !=" ")

if ( curdir:t == "v2") break \# just processed v2 dir?

if (-e scurdir/\$libext) then \# found some!

endif set libs $=$ (\$libs $/$ bin/ls $\$$ curdir/\$libext ')

end

set curdir = \$curdir:h

if ( \$?echocmd) echo done.

if $(\$ 1==" h i "||$ ?echocmd ) then

echo $\{\{0\}$ : Libraries found:

echo \$libs

goto alldone 
endif

set exec suffix = 'phigsx' \# distinguish executables

\# link with libraries

if ( $\$$ ?echocmd) echo \$CC \$PRE PHIGS LIB \$1.0।

\$CC \$PRE PHIGS LIB \$1.0 ।

\$libs \$trañs_lib \$global_lib \$POST_PHIGS_LIB -0 \$1.\$exec_suffix

if ( status $==\overline{0}$ ) then

if (\$?echocmd) echo./\$1

./\$1. Sexec suffix \# execute

else

echo $\$\{0\}$ : Fix link errors and try again.

endif goto alldone

\#\#\#\#\#\#\#\#\#\# \#

usage:

echo Usage: $\$ 0$ '[-v] file'

echo Note: file has no extension, but file.f or file.c exists.

echo ' $-v^{\prime}$ option will echo commands before they are executed.

goto alldone

\#\#\#\#\#\#\#\#\#\#\#

alldone:

exit 


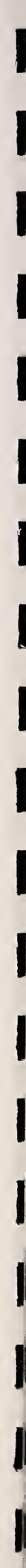


APPENDIX G

BUILDING THE PVT IN UNIX

The following steps must be followed in order to install the test suite on a UNIX system. Note that Makefiles have been provided that will run on most systems but may require modifications for your system.

No executable is shipped in the distribution; all programs must be regenerated. When building the f2c libraries, please read the section describing the use of \#define USE_STRLEN. This definition is crucial for some systems.

Step [1]

Build the f2c library - libf2c.a

(a)

cd pvt/E2C

(b)

Edit the makefile

* Check the description of onexit and how it applies to your system.

* Select the C compile line that you want (i.e. whether you want prototyping).

(c)

Review the README file and modify items as required by your system.

(d)

Type make

The result is a condensed version of the actual f2c utility. The libraries libF77 and libI77 have been combined into one library, and the actual translator $\mathrm{f} 2 \mathrm{C}$ is not distributed. All code has been translated and only the subroutine library is required. The code itself has not been altered in any way, and sites that have f2c installed as a system library may use their own version. 
Step [2] Build the C layer code library - layer.a

(a)

cd pvt/LAYER

(b)

Edit the Makefile

* Change PVTHOME (currently /home/kevin/pvt) to the directory where the PVT test suite is installed.

* Change XINCDIR (currently /usr/openwin/include ) to the directory where X11 is installed. This is required for include files if your implementation uses $\mathrm{X} 11$. If you do not require this variable, just leave it blank.

* Change PHIGSINCDIR (currently \$(PHIGSHOME)/include/phigs) to the directory where PHIGS is installed. This is required for include files.

* Select the C compile line that pertains to your system (i.e. whether you want prototyping)

(c)

Type make

Step [3] Build the subroutine library - libcpvt.a

The following steps build use the single directory, rather than hierarchically (see section 3.1.2).

(a)

cd pvt/V2IIB

(b)

Edit the Makefile

* Change PVTHOME (currently /home/kevin/pvt) to the directory where the PVT test suite is installed.

* Change XINCDIR (currently /usr/openwin/include ) to the directory where X11 is installed. This is required for include files if your implementation uses XI1. If you do not require this variable, just leave it blank.

* Change PHIGSINCDIR (currently \$(PHIGSHOME)/include/phigs) to the directory where PHIGS is installed. This is required for include files.

* Select the C compile line that pertains to your system (i.e. whether you want prototyping) 
(c)

Edit INITGL.C:

* Change the filename character variable on line 180 to the directory where you will install the configuration file using INITPH.

Currently:

S_copy (filenm, "INITPH\$DAT", 60I, 10L); Change to:

s_copy (filenm, "<your directory>/pvt/INITPH\$DAT", 60L, <NN>I Change the <NN> to the length of the character string, (up to a max of 60)

(d)

Edit MULTWS.c:

* Change the filename character variable on line 206 to the directory where you will install the configuration file using INITPH.

Currently:

S_copy (filenm, "INITPH\$DAT", 60L, 10L); Chañge to:

s_copy (filenm, "<your directory>/pvt/INITPH\$DAT", 60L, <NN>L Change the <NN> to the length of the character string, (up to a max of 60)

* Refer to the discussion on MULTwS in the customizations section (section 3.2.5) to determine if further changes are required (i.e. do you need to generate a special workstation type).

(e)

Edit XPOPPH.c:

* Refer to the discussion on XPOPPH in the customizations section (section 3.2.2) to determine if any changes are required (i.e. do you need to generate a special workstation type).

(f)

Type make

Step [4] Build initph and oprcmt

(a)

cd pvt

(b)

Edit the Makefile

* Change PVTHOME (currently /home/kevin/pvt) to the directory where the PVT test suite is installed. 
* Change XINCDIR (currently /usr/openwin/include) to the directory where X11 is installed. This is required for include files if your implementation uses X11. If you do not require this variable, just leave it blank.

* Make sure PHIGSHOME is set.

* Check the system variable CPHIGS LIB. It must be set to the libraries required by your PHIGS implementation, including the PHIGS libraries themselves (i.e. does it use any $x$ libraries?). set this variable accordingly to locate the libraries you will need.

(c)

Edit pvt/C/std/initph.c:

* Change the filename character variable on line 361 to the directory where you would like to have the configuration file installed.

Currently:

s copy (filenm, "INITPH\$DAT", 60L, 10L); Chañge to:

s_copy (filenm, "<your directory>/pvt/INITPH\$DAT", Change the <NN> to the length of the character string, (up to a max of 60)

[d] Change the filename character variable on line 363 to the directory where you would like to have the HUMAN-READABLE configuration file installed.

Currently:

S_copy (filenm, "INITPH\$PRT", 60L, 10L);

Change to:

S_copy (filenm, "<your directory>/pvt/INITPH\$PRT", 60L, <NN>L) Change the <NN $>$ to the length of the character string, (up to a max of 60 )

(e)

Type make cadmin

Step [5] run pvt/C/std/initph to create the configuration file, as described in section 4.2 .

Step [6] Build the PVT test suite.

[a] Edit pvt/Makefile:

* Select the C compile line that pertains to your system (i.e. whether you want prototyping)

In the PVT root type one of: 
make all

[Build the $\mathrm{C}$ test suite]

make cadmin [Build the $C$ version of initph]

Step [7] Run the test suite.

Each of the programs that exist in the sub-tree must be executed to perform a complete validation. You should develop procedures on your system to accomplish this efficiently. When the actual validation is performed, each program must compile, link and execute without error.

In the PVT root type one of:

make -f Makefile.run run [Run both active and passive $\mathrm{C}$ tests]

make -f Makefile.run runactive

[Run active C tests]

make -f Makefile.run runpassive

[Run passive C tests] 


\section{APPENDIX H}

SOETWARE METRICS

The size of the PVT system may be measured in several ways. The following table presents some size metrics for version 2.1 .

$\begin{array}{llr}\text { number of modules } & : & 86 \\ \text { number of test programs } & : & 479 \\ \text { number of lines in test programs } & : & 81,861 \\ \text { number of subroutine libraries } & : & 49 \\ \text { number of subroutines/functions } & : & 367 \\ \text { number of lines in subroutines/functions } & : 29,098 \\ \text { number of lines in system utilities } & : & 892 \\ \text { number of lines in module documentation } & : 83,177 \\ \text { number of lines in system documentation } & : 12,873 \\ \text { number of SRs (semantic requirements) } & : & 1,440 \\ \text { number of TCs (test cases) } & : & 2,915 \\ \text { staff-months of effort } & : & 115 \\ \text { (design, code, documentation) } & \end{array}$




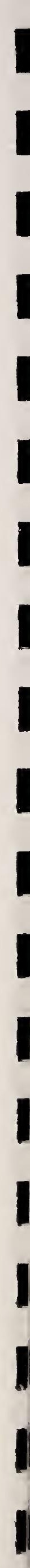




\title{
APPENDIX I
}

\author{
EUNCTION CROSS-REFERENCE
}

\#F 001 <open phigs>:

01/SR01 02.01.01/SR08 02.02.03/SR05 03.01/SR0 $403.02 / S R 01$

05.01.01/SR01 05.01.01/SR02 09.01.01.01/SR01 09.01.01.02/SR01

$11.02 /$ SR02 $11.02 /$ SR0 9

\#F $002<$ <lose phigs>:

01/SR02 09.01.01.01/SR04 09.01.01.02/SR04 11.02/SR02

\#F 003 <open workstation>:

$04.02 .01 .02 /$ SR24 $04.02 .01 .02 / S R 2504.02 .02 .02 / S R 24$

$04.02 .02 .02 /$ SR25 $04.02 .03 .02 /$ SR24 $04.02 .03 .02 /$ SR2 5

$04.02 .04 .02 /$ SR0 $904.02 .05 .02 /$ SR25 $04.02 .05 .02 /$ SR 26

$04.02 .05 .03 /$ SR2 $404.02 .05 .03 /$ SR25 $04.02 .05 .04 / S R 16$

$04.02 .05 .04 /$ SR17 $04.03 .02 .02 /$ SR13 $04.03 .02 .02 /$ SR1 4

$04.03 .02 .02 /$ SR20 $04.03 .04 .02 /$ SR03 $04.03 .04 .02 /$ SR0 $705.01 .01 /$ SR0 6

05.01.01/SR08 05.01.01/SR11 05.01.02/SR06 $05.02 /$ SR0 $805.02 / S R 09$

$05.03 /$ SR11 $06.02 .02 /$ SR09 $06.02 .02 /$ SR10 $06.02 .02 /$ SR1 1

06.02.02/SR19 06.03/SR05 09.01.01.01/SR02 09.01.01.02/SR02

$09.01 .02 .01 /$ SR01 09.01.02.01/SR02 09.01.02.01/SR03

$09.01 .02 .01 /$ SR05 $09.01 .02 .01 /$ SR12 $09.01 .02 .02 /$ SR01

$09.01 .02 .02 /$ SR02 $09.01 .02 .02 /$ SR03 $09.01 .02 .02 /$ SR0 5

$09.01 .02 .02 /$ SR12 $11.02 /$ SR02

\#F $004<$ close workstation>:

05.01.01/SR12 05.03/SR12 09.01.01.01/SR03 09.01.01.02/SR03

$09.01 .02 .01 /$ SR0 $409.01 .02 .02 /$ SR0 $411.02 /$ SR02

\#F 005 <redraw all structures>:

$05.02 /$ SR16 09.01.01.01/SR03 09.01.01.02/SR03 09.01.02.01/SR04

$09.01 .02 .01 /$ SR09 09.01.02.02/SR04 09.01.02.02/SR09

$09.01 .10 .01 /$ SR01 09.01.10.02/SR01 09.01.11.01/SR0 4

$09.01 .11 .02 /$ SR01 $11.02 /$ SR02

\#F 006 <update workstation>:

05.02/SR17 05.02/SR18 09.01.01.01/SR03 09.01.01.02/SR03

$09.01 .02 .01 /$ SR0 $409.01 .02 .01 /$ SR09 $09.01 .02 .02 / S R 04$

09.01.02.02/SR09 09.01.10.01/SR01 09.01.10.02/SR01

$09.01 .11 .01 /$ SR04 09.01.11.02/SR01 11.02/SR02 
\#F 007 <set display update state>:

05.02/SR0 105.02 /SR02 05.02/SR0 $305.02 /$ SR0 $8 \quad 05.02 / S R 2205.02 /$ SR2 3 $05.02 / S R 2405.02 / S R 25 \quad 05.02 / S R 26 \quad 05.02 / S R 27 \quad 05.02 / S R 28 \quad 05.02 / S R 29$ 05.02/SR31 05.02/SR32 09.01.01.01/SR03 09.01.01.02/SR03 $09.01 .02 .01 /$ SR0 $409.01 .02 .01 /$ SR0 $909.01 .02 .02 /$ SR0 4 $09.01 .02 .02 /$ SR09 $09.01 .10 .01 /$ SR01 09.01.10.02/SR01 $09.01 .11 .01 /$ SR0 $409.01 .11 .02 /$ SR01 $11.02 /$ SR02

\#F $008<$ message>:

09.01.01.01/SR03 09.01.01.02/SR03 09.01.02.01/SR0 4 $09.01 .02 .02 /$ SR0 $411.02 /$ SR0 2

\#F 009 <polyline 3>:

$02.03 .03 /$ SR0 $602.03 .03 /$ SR10 $02.03 .03 /$ SR12 $04.01 .01 /$ SR01

$04.01 .01 /$ SR07 $04.01 .03 /$ SR09 $04.01 .03 /$ SR11 $04.03 .04 .01 /$ SR15

$05.02 /$ SR1 $905.02 / S R 20 \quad 05.02 / S R 2106.01 .02 / S R 1706.01 .02 / S R 57$ $09.01 .01 .01 /$ SR05 09.01.01.02/SR05 09.01.10.01/SR03

$09.01 .10 .02 / \mathrm{SR} 0311.02 / \mathrm{SR} 02$

\#F 010 <polyline>:

$02.03 .03 /$ SR0 $602.03 .03 /$ SR10 $02.03 .03 /$ SR12 $04.01 .01 /$ SR0 4

$04.01 .01 /$ SR07 $04.01 .03 /$ SR09 $04.01 .03 /$ SR10 $04.03 .04 .01 /$ SR15

$05.02 /$ SR19 $05.02 /$ SR20 $05.02 /$ SR21 $06.01 .02 /$ SR17 $06.01 .02 /$ SR5 7 $09.01 .01 .01 /$ SR05 09.01.01.02/SR05 09.01.10.01/SR03 $09.01 .10 .02 /$ SR03 $11.02 /$ SR0 2

\#F 011 <polymarker 3>:

$02.03 .03 /$ SR0 $602.03 .03 /$ SR10 $02.03 .03 /$ SR11 $04.01 .02 /$ SR0 1 $04.01 .02 /$ SR07 $04.03 .04 .01 /$ SR15 $05.02 /$ SR19 $05.02 /$ SR20 $05.02 / S R 21$ $06.01 .02 /$ SR17 $06.01 .02 /$ SR57 $06.01 .02 /$ SR59 $09.01 .01 .01 /$ SR0 5 09.01.01.02/SR05 09.01.10.01/SR03 09.01.10.02/SR03 11.02/SR02

\#E 012 <polymarker>:

$02.03 .03 /$ SR0 $602.03 .03 /$ SR10 $02.03 .03 /$ SR11 $04.01 /$ SR0 1

$04.01 .02 /$ SR0 $404.01 .02 /$ SR0 $704.03 .04 .01 /$ SR $1505.02 /$ SR 19

$05.02 / S R 2005.02 / S R 2106.01 .02 / S R 17 \quad 06.01 .02 / S R 5706.01 .02 / S R 59$ $09.01 .01 .01 /$ SR05 09.01.01.02/SR05 09.01.10.01/SR03

$09.01 .10 .02 / \mathrm{SR} 0311.02 / \mathrm{SR} 02$

\#F $013<$ text $3>$ :

$02.03 .03 /$ SR0 $602.03 .03 /$ SR10 $02.03 .03 /$ SR15 $04.01 .03 /$ SR01

$04.02 .03 .01 / \mathrm{SR} 0104.02 .03 .01 / \mathrm{SR} 02 \quad 04.03 .04 .01 / \mathrm{SR} 15 \quad 05.02 / \mathrm{SR} 19$

$05.02 / S R 20 \quad 05.02 / S R 2106.01 .02 / S R 1706.01 .02 / S R 5706.01 .02 / S R 60$ 09.01.01.01/SR05 09.01.01.02/SR05 11.02/SR02

\section{\#F $014<$ <text>:}

$02.03 .03 /$ SR0 $602.03 .03 /$ SR10 $02.03 .03 /$ SR15 $04.01 .03 /$ SR0 4

$04.02 .03 .01 / S R 0104.02 .03 .01 /$ SR02 $04.03 .04 .01 /$ SR15 $05.02 /$ SR 19

$05.02 / S R 2005.02 / S R 2106.01 .02 / S R 1706.01 .02 / S R 5706.01 .02 / S R 60$ 09.01.01.01/SR05 09.01.01.02/SR05 $11.02 /$ SR02

\#F 015 <annotation text relative $3>$ :

$02.03 .03 /$ SR0 $602.03 .03 /$ SR10 02.03.03/SR16 04.01.04/SR01 
$04.01 .04 / S R 0704.01 .04 / S R 0904.02 .04 .01 / S R 0104.02 .04 .01 / S R 02$

$04.03 .04 .01 /$ SR15 $05.02 /$ SR19 $05.02 /$ SR20 $05.02 / S R 2106.01 .02 / S R 17$

$06.01 .02 /$ SR57 $06.01 .02 /$ SR61 $06.01 .02 /$ SR62 $09.01 .01 .01 /$ SR0 5

$09.01 .01 .02 /$ SR0 $511.02 /$ SR02

\#F 016 <annotation text relative>:

$02.03 .03 /$ SR0 $602.03 .03 /$ SR10 02.03.03/SR16 $04.01 .04 /$ SR0 4

$04.01 .04 /$ SR07 $04.01 .04 /$ SR0 8 04.01.04/SR09 $04.02 .04 .01 /$ SR01

$04.02 .04 .01 /$ SR02 $04.03 .04 .01 /$ SR15 $05.02 /$ SR19 $05.02 /$ SR2 0

05.02/SR21 06.01.02/SR17 06.01.02/SR57 06.01.02/SR61

$06.01 .02 /$ SR62 09.01.01.01/SR05 09.01.01.02/SR05 $11.02 /$ SR02

\#F $017<$ fill area $3>$ :

$02.03 .03 /$ SR0 $602.03 .03 /$ SR10 $02.03 .03 /$ SR13 $04.01 .05 /$ SR01

$04.01 .05 /$ SR0 $704.01 .05 /$ SR0 $804.03 .04 .01 /$ SR15 $05.02 /$ SR1 9

$05.02 /$ SR2 $05.02 /$ SR2 $106.01 .02 /$ SR1 $706.01 .02 /$ SR5 $706.01 .02 / S R 63$

$09.01 .01 .01 /$ SR05 $09.01 .01 .02 /$ SR0 $509.01 .10 .01 /$ SR03

$09.01 .10 .02 /$ SR03 $11.02 /$ SR02

\#F 018 <fill area>:

$02.03 .03 /$ SR0 $602.03 .03 /$ SR10 $02.03 .03 /$ SR13 $04.01 .05 /$ SR0 4

$04.01 .05 /$ SR07 $04.01 .05 /$ SR0 $804.03 .04 .01 /$ SR15 $05.02 /$ SR1 9

05.02/SR20 05.02/SR21 06.01.02/SR17 06.01.02/SR57 06.01.02/SR63

$09.01 .01 .01 /$ SR05 $09.01 .01 .02 /$ SR05 $09.01 .10 .01 /$ SR0 3

$09.01 .10 .02 /$ SR03 $11.02 /$ SR02

\#F 019 <fill area set $3>$ :

$02.03 .03 /$ SR0 $602.03 .03 /$ SR10 $02.03 .03 /$ SR14 $04.01 .06 /$ SR0 1

$04.01 .06 /$ SR07 $04.01 .06 /$ SR0 8 04.03.04.01/SR15 $05.02 /$ SR19

$05.02 / S R 20 \quad 05.02 / S R 2106.01 .02 / S R 1706.01 .02 / S R 5706.01 .02 / S R 63$

06.01.02/SR64 09.01.01.01/SR05 09.01.01.02/SR05 09.01.10.01/SR04

$09.01 .10 .02 /$ SR0 $411.02 / S R 02$

\#F $020<$ <ill area set>:

$02.03 .03 /$ SR0 $602.03 .03 /$ SR10 $02.03 .03 /$ SR14 $04.01 .06 /$ SR0 4

$04.01 .06 /$ SR0 $704.01 .06 /$ SR0 $804.03 .04 .01 /$ SR15 $05.02 /$ SR19

$05.02 / S R 20 \quad 05.02 / S R 2106.01 .02 /$ SR $1706.01 .02 / S R 5706.01 .02 / S R 63$

06.01.02/SR64 09.01.01.01/SR05 09.01.01.02/SR05 09.01.10.01/SR04 $09.01 .10 .02 /$ SR0 $411.02 / S R 02$

\#F $021<$ cell array 3>:

$02.03 .03 /$ SR0 $602.03 .03 /$ SR10 $02.03 .03 /$ SR17 $04.01 .07 /$ SR01

04.01.07/SR04 04.01.07/SR05 04.01.07/SR11 04.01.07/SR12

$04.01 .07 /$ SR13 $04.01 .07 /$ SR14 $04.03 .04 .01 /$ SR15 $05.02 /$ SR1 9

$05.02 /$ SR20 05.02/SR21 $06.01 .02 /$ SR17 $06.01 .02 /$ SR5 7

$09.01 .01 .01 /$ SR05 09.01.01.02/SR05 09.01.03.01/SR16

$09.01 .03 .02 /$ SR16 11.02/SR02

\#F $022<$ cell array>:

02.03.03/SR06 02.03.03/SR10 02.03.03/SR17 $04.01 .07 /$ SR0 6

04.01.07/SR09 04.01.07/SR10 04.01.07/SR11 $04.01 .07 /$ SR12

$04.01 .07 /$ SR13 $04.01 .07 /$ SR14 $04.03 .04 .01 /$ SR15 $05.02 /$ SR1 9

$05.02 /$ SR20 05.02/SR21 $06.01 .02 /$ SR17 $06.01 .02 / S R 57$

$09.01 .01 .01 /$ SR0 $509.01 .01 .02 /$ SR0 $509.01 .03 .01 /$ SR 16 
\#F 023 <generalized drawing primitive $3>$ : 02.03.03/SR06 02.03.03/SR10 04.01.08/SR01 04.03.04.01/SR15 $05.02 /$ SR19 $05.02 / S R 20 \quad 05.02 / S R 2106.01 .02 / S R 1706.01 .02 / S R 57$ $09.01 .01 .01 / \mathrm{SR} 0509.01 .01 .02 / \mathrm{SR} 05$ 09.01.10.01/SR03 $09.01 .10 .02 / \mathrm{SR} 0311.02 / \mathrm{SR} 02$

\#F 024 <generalized drawing primitive>:

$02.03 .03 /$ SR0 $602.03 .03 /$ SR10 04.01.08/SR0 $604.03 .04 .01 /$ SR15

$05.02 / S R 1905.02 / S R 20 \quad 05.02 / S R 2106.01 .02 / S R 1706.01 .02 / S R 57$ 09.01.01.01/SR05 09.01.01.02/SR05 09.01.10.01/SR03 $09.01 .10 .02 /$ SR03 $11.02 /$ SR02

\#F 025 <set polyline index>:

$04.02 .01 .02 /$ SR03 $04.02 .01 .02 /$ SR0 $404.02 .01 .02 /$ SR0 5 $04.02 .01 .02 /$ SR0 $604.02 .01 .02 /$ SR0 $04.02 .01 .02 / S R 35$ $04.02 .01 .02 / S R 4304.02 .01 .02 / S R 44 \quad 04.02 .01 .02 / S R 45$ $04.02 .01 .02 / S R 47 \quad 05.02 / S R 19 \quad 05.02 / S R 20 \quad 05.02 / S R 21$ $09.01 .01 .01 /$ SR05 $09.01 .01 .02 /$ SR05 $09.01 .03 .01 / S R 01$ $09.01 .03 .02 /$ SR01 $11.02 /$ SR0 2

\#F 026 <set polymarker index>: $04.02 .02 .02 /$ SR0 $304.02 .02 .02 /$ SR0 $404.02 .02 .02 /$ SR0 5 $04.02 .02 .02 /$ SR0 $604.02 .02 .02 /$ SR0 $704.02 .02 .02 /$ SR35 $04.02 .02 .02 / \mathrm{SR} 4304.02 .02 .02 / \mathrm{SR} 44 \quad 04.02 .02 .02 / \mathrm{SR} 45$ $04.02 .02 .02 / S R 4604.02 .02 .02 / S R 48 \quad 05.02 / S R 1905.02 / S R 20$ $05.02 / S R 2109.01 .01 .01 /$ SR05 $09.01 .01 .02 /$ SR05 $09.01 .03 .01 / S R 01$ $09.01 .03 .02 /$ SR01 $11.02 /$ SR02

\#F 027 <set text index>:

$04.02 .03 .02 /$ SR03 $04.02 .03 .02 /$ SR0 $404.02 .03 .02 /$ SR0 5

$04.02 .03 .02 /$ SR0 $604.02 .03 .02 /$ SR0 $704.02 .03 .02 /$ SR3 8

$04.02 .03 .02 /$ SR39 $04.02 .03 .02 /$ SR5 $704.02 .03 .02 /$ SR61

$04.02 .03 .02 /$ SR $6404.02 .04 .02 /$ SR01 $04.02 .04 .02 /$ SR0 2

$04.02 .04 .02 /$ SR0 $304.02 .04 .02 /$ SR0 $404.02 .04 .02 /$ SR0 5

$04.02 .04 .02 /$ SR11 $04.02 .04 .02 /$ SR12 $04.02 .04 .02 / S R 24$

$04.02 .04 .02 /$ SR2 $704.02 .04 .02 /$ SR29 $05.02 /$ SR $1905.02 /$ SR2 0

$05.02 / S R 2109.01 .01 .01 /$ SR05 $09.01 .01 .02 /$ SR05 $09.01 .03 .01 / S R 01$ $09.01 .03 .02 /$ SR01 $11.02 /$ SR02

\#F 028 <set interior index>:

$04.02 .05 .02 /$ SR0 $304.02 .05 .02 /$ SR0 $404.02 .05 .02 /$ SR0 5 $04.02 .05 .02 /$ SR0 $604.02 .05 .02 /$ SR0 $704.02 .05 .02 /$ SR0 8 $04.02 .05 .02 / S R 3504.02 .05 .02 /$ SR39 $04.02 .05 .02 / S R 5105.02 / S R 19$ 05.02/SR20 05.02/SR21 09.01.01.01/SR05 09.01.01.02/SR05 $09.01 .03 .01 /$ SR01 09.01.03.02/SR01 11.02/SR02

\#F 029 <set edge index>:

$04.02 .05 .03 /$ SR0 $304.02 .05 .03 /$ SR0 $404.02 .05 .03 /$ SR0 5

$04.02 .05 .03 /$ SR0 $64.02 .05 .03 /$ SR0 $704.02 .05 .03 /$ SR30

$04.02 .05 .03 /$ SR3 $84.02 .05 .03 /$ SR $46 \quad 04.02 .05 .03 /$ SR 47

$04.02 .05 .03 /$ SR $4804.02 .05 .03 /$ SR50 $05.02 /$ SR19 $05.02 /$ SR 20

05.02/SR21 09.01.01.01/SR05 09.01.01.02/SR05 09.01.03.01/SR01 
$09.01 .03 .02 /$ SR01 $11.02 /$ SR02

\#F $030<$ set linetype>:

$04.02 .01 .01 /$ SR03 $04.02 .01 .01 /$ SR0 $404.02 .01 .01 /$ SR0 5

$04.02 .01 .01 /$ SR0 $604.02 .01 .01 /$ SR0 $0505.02 /$ SR19 $05.02 /$ SR2 0

$05.02 /$ SR21 09.01.01.01/SR05 09.01.01.02/SR05 11.02/SR02

\#F 031 <set linewidth scale factor>:

$04.02 .01 .01 /$ SR03 $04.02 .01 .01 /$ SR13 04.02.01.01/SR14

$04.02 .01 .01 /$ SR15 $04.02 .01 .01 /$ SR16 $05.02 /$ SR19 $05.02 /$ SR20

$05.02 /$ SR21 09.01.01.01/SR05 09.01.01.02/SR05 $11.02 /$ SR02

\#F 032 <set polyline colour index>:

$04.02 .01 .01 /$ SR03 $04.02 .01 .01 /$ SR19 $04.02 .01 .01 /$ SR20

$04.02 .01 .01 / S R 2104.02 .01 .01 / S R 2205.02 / S R 1905.02 / S R 20$

$05.02 /$ SR2 $109.01 .01 .01 /$ SR05 $09.01 .01 .02 /$ SR05 $09.01 .03 .01 / S R 12$

$09.01 .03 .02 /$ SR12 $11.02 /$ SR02

\#F 033 <set marker type>:

$04.02 .02 .01 /$ SR03 $04.02 .02 .01 /$ SR0 $404.02 .02 .01 /$ SR0 5

$04.02 .02 .01 /$ SR0 $64.02 .02 .01 /$ SR0 $0404.02 .02 .01 /$ SR $1705.02 / S R 19$

$05.02 /$ SR20 $05.02 /$ SR21 $09.01 .01 .01 /$ SR05 $09.01 .01 .02 /$ SR0 5

$11.02 /$ SR02

\#F 034 <set marker size scale factor>:

$04.02 .02 .01 /$ SR03 $04.02 .02 .01 /$ SR13 $04.02 .02 .01 /$ SR14

$04.02 .02 .01 /$ SR15 $04.02 .02 .01 /$ SR16 $04.02 .02 .01 /$ SR17 $05.02 /$ SR1 9

$05.02 /$ SR20 05.02/SR21 09.01.01.01/SR0 $509.01 .01 .02 /$ SR0 5

$11.02 /$ SR02

\#F 035 <set polymarker colour index>:

$04.02 .02 .01 /$ SR03 $04.02 .02 .01 /$ SR20 $04.02 .02 .01 /$ SR2 1

$04.02 .02 .01 / S R 2204.02 .02 .01 / S R 2305.02 / S R 1905.02 / S R 20$

$05.02 /$ SR21 09.01.01.01/SR05 09.01.01.02/SR05 09.01.03.01/SR12 $09.01 .03 .02 /$ SR12 $11.02 /$ SR02

\#F 036 <set text font>:

$04.02 .03 .01 /$ SR11 $04.02 .03 .01 /$ SR12 $04.02 .03 .01 /$ SR13

$04.02 .03 .01 /$ SR21 $04.02 .03 .01 /$ SR22 $04.02 .04 .01 /$ SR0 5

$04.02 .04 .01 /$ SR0 $604.02 .04 .01 /$ SR14 $04.02 .04 .01 /$ SR15 $05.02 / S R 19$

$05.02 /$ SR20 05.02/SR21 09.01.01.01/SR05 09.01.01.02/SR0 5

$11.02 /$ SR02

\#F 037 <set text precision>:

$04.02 .03 .01 /$ SR11 $04.02 .03 .01 /$ SR25 $04.02 .03 .01 /$ SR2 6

$04.02 .03 .01 /$ SR2 $704.02 .03 .01 /$ SR2 $804.02 .03 .01 /$ SR2 9

$04.02 .03 .01 /$ SR30 $04.02 .03 .01 /$ SR31 $04.02 .04 .01 /$ SR0 5

$04.02 .04 .01 /$ SR18 $04.02 .04 .01 /$ SR19 $04.02 .04 .01 /$ SR2 0

$04.02 .04 .01 /$ SR21 $04.02 .04 .01 /$ SR22 $04.02 .04 .01 /$ SR23 $05.02 / S R 19$

$05.02 / S R 2005.02 / S R 2109.01 .01 .01 /$ SR0 $509.01 .01 .02 /$ SR0 5

$09.01 .10 .01 /$ SR01 $09.01 .10 .02 /$ SR01 09.01.11.01/SR0 4

$09.01 .11 .02 /$ SR01 $11.02 /$ SR02

\#F 038 <set character expansion factor>: 
$04.02 .03 .01 /$ SR11 $04.02 .03 .01 /$ SR34 $04.02 .03 .01 /$ SR3 5

$04.02 .03 .01 /$ SR37 $04.02 .04 .01 /$ SR0 $504.02 .04 .01 /$ SR2 6

$04.02 .04 .01 /$ SR29 $05.02 /$ SR19 $05.02 /$ SR20 $05.02 / S R 21$

$09.01 .01 .01 /$ SR05 09.01.01.02/SR05 11.02/SR02

\#F 039 <set character spacing>:

$04.02 .03 .01 /$ SR11 $04.02 .03 .01 /$ SR40 $04.02 .03 .01 /$ SR 41

$04.02 .03 .01 / S R 4304.02 .04 .01 / S R 0504.02 .04 .01 / S R 32$

$04.02 .04 .01 /$ SR34 $05.02 /$ SR19 $05.02 /$ SR20 $05.02 / S R 21$

09.01.01.01/SR05 09.01.01.02/SR05 11.02/SR02

\#E 040 <set text colour index>:

$04.02 .03 .01 /$ SR11 $04.02 .03 .01 /$ SR4 $604.02 .03 .01 /$ SR 47

$04.02 .03 .01 /$ SR4 $804.02 .03 .01 /$ SR4 $904.02 .04 .01 /$ SR0 5

$04.02 .04 .01 /$ SR37 $04.02 .04 .01 /$ SR3 $804.02 .04 .01 /$ SR39 $05.02 / S R 19$

$05.02 /$ SR20 05.02/SR21 09.01.01.01/SR05 $09.01 .01 .02 /$ SR0 5

$09.01 .03 .01 /$ SR $1209.01 .03 .02 /$ SR $1211.02 /$ SR0 2

\#F 041 <set character height>:

$04.02 .03 .01 /$ SR03 $04.02 .03 .01 /$ SR11 $04.02 .03 .01 /$ SR52

$04.02 .03 .01 /$ SR58 $04.02 .03 .01 /$ SR61 $05.02 /$ SR19 $05.02 /$ SR20

$05.02 / S R 2109.01 .01 .01 / S R 0509.01 .01 .02 / S R 0511.02 / S R 02$

\#F 042 <set character up vector>:

$04.02 .03 .01 /$ SR11 $04.02 .03 .01 /$ SR79 $04.02 .03 .01 /$ SR8 0

$04.02 .03 .01 /$ SR81 $05.02 /$ SR19 $05.02 / S R 20 \quad 05.02 / S R 21$

$09.01 .01 .01 /$ SR05 $09.01 .01 .02 /$ SR05 $11.02 /$ SR02

\#F 043 <set text path>:

$04.02 .03 .01 /$ SR11 $04.02 .03 .01 /$ SR64 $04.02 .03 .01 /$ SR65

$04.02 .03 .01 /$ SR $6604.02 .03 .01 /$ SR $6704.02 .03 .01 /$ SR 68

$04.02 .03 .01 /$ SR69 $05.02 /$ SR19 $05.02 /$ SR20 $05.02 /$ SR21

09.01.01.01/SR05 09.01.01.02/SR05 09.01.10.01/SR01

$09.01 .10 .02 /$ SR01 09.01.11.01/SR0 4 09.01.11.02/SR01 $11.02 /$ SR0 2

\#F 044 <set text alignment>:

$04.02 .03 .01 /$ SR11 $04.02 .03 .01 /$ SR72 $04.02 .03 .01 /$ SR73

$04.02 .03 .01 /$ SR74 $04.02 .03 .01 /$ SR75 $04.02 .03 .01 /$ SR76 $05.02 / S R 19$

$05.02 / S R 20 \quad 05.02 / S R 2109.01 .01 .01 /$ SR0 $509.01 .01 .02 /$ SR0 5

$09.01 .10 .01 /$ SR01 09.01.10.02/SR01 09.01.11.01/SR0 4

$09.01 .11 .02 /$ SR01 11.02/SR02

\#F 045 <set annotation text character height>:

$04.02 .04 .01 /$ SR0 $504.02 .04 .01 /$ SR42 $04.02 .04 .01 / S R 48$

$04.02 .04 .01 /$ SR51 $04.02 .04 .01 / S R 52 \quad 05.02 / S R 1905.02 / S R 20$

$05.02 /$ SR21 09.01.01.01/SR05 09.01.01.02/SR05 $11.02 /$ SR02

\#F 046 <set annotation text character up vector>: $04.02 .04 .01 /$ SR05 $04.02 .04 .01 /$ SR70 04.02.04.01/SR71

$04.02 .04 .01 /$ SR72 $05.02 /$ SR19 $05.02 / S R 20 \quad 05.02 / S R 21$

$09.01 .01 .01 /$ SR0 $509.01 .01 .02 /$ SR05 11.02/SR02

\#F 047 <set annotation text path>:

$04.02 .04 .01 /$ SR05 $04.02 .04 .01 /$ SR55 $04.02 .04 .01 /$ SR5 6 
$04.02 .04 .01 / \mathrm{SR} 57 \quad 04.02 .04 .01 / \mathrm{SR} 58 \quad 04.02 .04 .01 / \mathrm{SR} 59$

$04.02 .04 .01 /$ SR60 $05.02 / S R 1905.02 / S R 20 \quad 05.02 / S R 21$

$09.01 .01 .01 /$ SR05 09.01.01.02/SR05 09.01.10.01/SR01

09.01.10.02/SR01 09.01.11.01/SR04 09.01.11.02/SR01 $11.02 /$ SR02

\#F 048 <set annotation text alignment>:

$04.02 .04 .01 /$ SR0 $504.02 .04 .01 /$ SR63 $04.02 .04 .01 /$ SR 64

$04.02 .04 .01 /$ SR $6504.02 .04 .01 /$ SR $66 \quad 04.02 .04 .01 /$ SR $67 \quad 05.02 / S R 19$

05.02/SR20 05.02/SR21 09.01.01.01/SR05 09.01.01.02/SR05

$09.01 .10 .01 /$ SR01 09.01.10.02/SR01 09.01.11.01/SR0 4

$09.01 .11 .02 /$ SR01 $11.02 /$ SR02

\#F 049 <set annotation style>:

$04.02 .04 .01 /$ SR05 $04.02 .04 .01 /$ SR75 $04.02 .04 .01 /$ SR7 6

$04.02 .04 .01 /$ SR78 $04.02 .04 .01 /$ SR79 $04.02 .04 .01 /$ SR80

$04.02 .04 .01 /$ SR81 $04.02 .04 .01 /$ SR82 $05.02 /$ SR19 $05.02 /$ SR20

05.02/SR21 09.01.01.01/SR05 09.01.01.02/SR05 $11.02 /$ SR0 2

\#F $050<$ set interior style>:

$04.02 .05 .01 /$ SR03 $04.02 .05 .01 /$ SR0 $404.02 .05 .01 /$ SR0 5

$04.02 .05 .01 /$ SR0 $604.02 .05 .01 /$ SR0 $0404.02 .05 .01 /$ SR0 8

$04.02 .05 .01 /$ SR09 $04.02 .05 .01 /$ SR10 $04.02 .05 .01 /$ SR12

$04.02 .05 .01 /$ SR13 $04.02 .05 .01 /$ SR14 $04.02 .05 .01 /$ SR15 $05.02 / S R 19$

05.02/SR20 05.02/SR21 09.01.01.01/SR05 09.01.01.02/SR05

$09.01 .10 .01 /$ SR01 09.01.10.02/SR01 09.01.11.01/SR0 4

$09.01 .11 .02 /$ SR01 $11.02 /$ SR02

\#F 051 <set interior style index>:

$04.02 .05 .01 /$ SR03 $04.02 .05 .01 /$ SR0 $404.02 .05 .01 /$ SR1 4

$04.02 .05 .01 /$ SR15 $04.02 .05 .01 /$ SR18 $04.02 .05 .01 /$ SR1 9

$04.02 .05 .01 / S R 2004.02 .05 .01 / S R 2105.02 / S R 1905.02 / S R 20$

$05.02 / S R 2109.01 .01 .01 /$ SR05 09.01.01.02/SR05 $11.02 /$ SR02

\#F 052 <set interior colour index>:

$04.02 .05 .01 /$ SR03 $04.02 .05 .01 /$ SR0 $404.02 .05 .01 /$ SR 10

$04.02 .05 .01 /$ SR13 $04.02 .05 .01 /$ SR14 $04.02 .05 .01 /$ SR2 6

$04.02 .05 .01 /$ SR27 $04.02 .05 .01 /$ SR28 $04.02 .05 .01 /$ SR2 $905.02 / S R 19$

$05.02 / S R 2005.02 / S R 2109.01 .01 .01 / S R 0509.01 .01 .02 / S R 05$

$09.01 .03 .01 /$ SR12 $09.01 .03 .02 /$ SR12 $11.02 /$ SR02

\#F 053 <set edge flag>:

$04.02 .05 .01 /$ SR0 $404.02 .05 .01 /$ SR32 $04.02 .05 .01 /$ SR33

$04.02 .05 .01 / S R 34 \quad 04.02 .05 .01 / S R 3504.02 .05 .01 / S R 36 \quad 05.02 / S R 19$

$05.02 / S R 20 \quad 05.02 / S R 2109.01 .01 .01 / S R 0509.01 .01 .02 / S R 05$

$09.01 .10 .01 /$ SR01 09.01.10.02/SR01 09.01.11.01/SR0 4

$09.01 .11 .02 /$ SR01 $11.02 /$ SR02

\#F 054 <set edgetype>:

$04.02 .05 .01 /$ SR0 $404.02 .05 .01 / S R 40 \quad 04.02 .05 .01 / S R 41$

$04.02 .05 .01 / S R 4204.02 .05 .01 / S R 4305.02 / S R 1905.02 / S R 20$

$05.02 / S R 2109.01 .01 .01 / S R 0509.01 .01 .02 / S R 0511.02 / S R 02$

\#F 055 <set edgewidth scale factor>:

$04.02 .05 .01 /$ SR0 $404.02 .05 .01 /$ SR49 $04.02 .05 .01 /$ SR50 
04.02.05.01/SR51 $04.02 .05 .01 /$ SR52 $05.02 /$ SR19 $05.02 /$ SR20 05.02/SR21 09.01.01.01/SR05 09.01.01.02/SR05 11.02/SR02

\#F 056 <set edge colour index>:

$04.02 .05 .01 /$ SR0 $404.02 .05 .01 /$ SR5 $504.02 .05 .01 /$ SR5 6

$04.02 .05 .01 /$ SR5 $704.02 .05 .01 /$ SR5 $85.02 /$ SR19 $05.02 /$ SR20

05.02/SR21 09.01.01.01/SR05 09.01.01.02/SR05 09.01.03.01/SR12 $09.01 .03 .02 / \mathrm{SR} 1211.02 / \mathrm{SR} 02$

\#F 057 <set pattern size>:

$04.02 .05 .01 /$ SR03 $04.02 .05 .01 /$ SR0 $404.02 .05 .01 / S R 61$

$04.02 .05 .01 /$ SR $6204.02 .05 .01 /$ SR63 $04.02 .05 .01 / S R 71$

$04.02 .05 .01 / S R 7304.02 .05 .01 /$ SR74 $04.02 .05 .01 / S R 76$

$04.02 .05 .01 /$ SR77 $05.02 /$ SR19 $05.02 /$ SR20 $05.02 / S R 21$

$09.01 .01 .01 /$ SR05 $09.01 .01 .02 /$ SR05 $11.02 /$ SR02

\#F 058 <set pattern reference point and vectors>:

$04.02 .05 .01 /$ SR03 $04.02 .05 .01 /$ SR0 $404.02 .05 .01 /$ SR 66

$04.02 .05 .01 /$ SR68 $04.02 .05 .01 /$ SR70 $04.02 .05 .01 /$ SR71

$04.02 .05 .01 / S R 7204.02 .05 .01 / S R 73 \quad 04.02 .05 .01 / S R 74$

$04.02 .05 .01 /$ SR76 $04.02 .05 .01 /$ SR77 $05.02 /$ SR19 $05.02 /$ SR20

05.02/SR21 09.01.01.01/SR05 09.01.01.02/SR05 $11.02 /$ SR02

\#F 059 <set pattern reference point>:

$04.02 .05 .01 /$ SR03 04.02.05.01/SR0 4 04.02.05.01/SR67

$04.02 .05 .01 /$ SR69 $04.02 .05 .01 /$ SR71 $04.02 .05 .01 / S R 72$

$04.02 .05 .01 /$ SR73 $04.02 .05 .01 /$ SR74 $04.02 .05 .01 /$ SR7 6

$04.02 .05 .01 /$ SR77 $05.02 /$ SR19 $05.02 /$ SR20 $05.02 / S R 21$

09.01.01.01/SR05 09.01.01.02/SR05 11.02/SR02

\#F 060 <add names to set>:

$02.03 .03 /$ SR0 $602.03 .03 /$ SR18 $02.03 .03 /$ SR19 $02.03 .03 /$ SR20

$04.03 .04 .01 /$ SR01 $04.03 .04 .01 /$ SR0 $904.03 .04 .01 / S R 10$

$04.03 .04 .01 /$ SR12 $04.03 .04 .01 /$ SR15 $04.03 .04 .01 /$ SR1 6

$04.03 .04 .02 /$ SR0 $404.03 .04 .02 /$ SR0 $804.03 .04 .02 /$ SR09 $05.02 / S R 19$

$05.02 / S R 2005.02 / S R 2109.01 .01 .01 / S R 05 \quad 09.01 .01 .02 / S R 05$

$09.01 .10 .01 /$ SR03 09.01.10.02/SR03 11.02/SR02

\#F 061 <remove names from set>:

$02.03 .03 /$ SR0 $602.03 .03 /$ SR1 $8 \quad 02.03 .03 /$ SR19 $02.03 .03 /$ SR20

$04.03 .04 .01 / \mathrm{SR} 0404.03 .04 .01 / \mathrm{SR} 0904.03 .04 .01 / \mathrm{SR} 10$

$04.03 .04 .01 /$ SR13 $04.03 .04 .01 /$ SR15 $04.03 .04 .01 /$ SR1 6

$04.03 .04 .02 /$ SR0 $404.03 .04 .02 /$ SR0 8 04.03.04.02/SR0 $905.02 / S R 19$

$05.02 / S R 2005.02 / S R 2109.01 .01 .01 /$ SR0 $509.01 .01 .02 /$ SR0 5

$09.01 .10 .01 /$ SR03 09.01.10.02/SR03 11.02/SR02

\#F 062 <set individual asf>:

$04.02 .01 .02 /$ SR35 $04.02 .01 .02 / S R 4304.02 .01 .02 / S R 44$

$04.02 .01 .02 /$ SR $4504.02 .01 .02 / S R 47 \quad 04.02 .02 .02 / S R 35$

$04.02 .02 .02 /$ SR $4304.02 .02 .02 / S R 44 \quad 04.02 .02 .02 / S R 45$

$04.02 .02 .02 /$ SR $4604.02 .02 .02 / S R 48 \quad 04.02 .03 .02 / S R 38$

$04.02 .03 .02 /$ SR39 $04.02 .03 .02 /$ SR5 $704.02 .03 .02 /$ SR 61

$04.02 .03 .02 /$ SR64 $04.02 .04 .02 /$ SR11 $04.02 .04 .02 /$ SR1 2

$04.02 .04 .02 /$ SR2 $404.02 .04 .02 / S R 27 \quad 04.02 .04 .02 / S R 29$ 
$04.02 .05 .02 /$ SR35 $04.02 .05 .02 /$ SR39 $04.02 .05 .02 /$ SR5 1 $04.02 .05 .03 / S R 3004.02 .05 .03 / S R 3804.02 .05 .03 / S R 46$ $04.02 .05 .03 /$ SR4 $704.02 .05 .03 /$ SR4 $8 \quad 04.02 .05 .03 /$ SR50 $04.03 .01 / S R 01$ $04.03 .01 /$ SR0 $404.03 .01 /$ SR05 $04.03 .01 /$ SR0 $604.03 .01 /$ SR0 7 $04.03 .01 /$ SR0 $84.03 .01 /$ SR0 $905.02 /$ SR1 $905.02 / S R 20 \quad 05.02 / S R 21$ 09.01.01.01/SR05 09.01.01.02/SR05 09.01.10.01/SR01 $09.01 .10 .02 /$ SR01 09.01.11.01/SR04 09.01.11.02/SR01 $11.02 /$ SR02

\#F 063 <set polyline representation>:

$04.02 .01 .02 /$ SR10 $04.02 .01 .02 /$ SR11 $04.02 .01 .02 /$ SR18 $04.02 .01 .02 /$ SR33 $04.02 .01 .02 /$ SR34 $04.02 .01 .02 /$ SR35 $04.02 .01 .02 / S R 4104.02 .01 .02 / S R 42 \quad 04.02 .01 .02 / S R 43$ $04.02 .01 .02 /$ SR4 $404.02 .01 .02 /$ SR4 $5 \quad 04.02 .01 .02 / S R 46$ $04.02 .01 .02 / S R 4705.02 / S R 1905.02 / S R 2005.02 / S R 21$ 09.01.01.01/SR03 09.01.01.02/SR03 09.01.02.01/SR0 4 $09.01 .02 .01 /$ SR0 9 $09.01 .03 .01 /$ SR01 $09.01 .03 .01 / \mathrm{SR} 12$ $09.01 .02 .02 /$ SR0 4 $09.01 .03 .01 / \mathrm{SR} 03$ $09.01 .03 .02 /$ SR01 $09.01 .02 .02 / \mathrm{SR} 09$ $09.01 .03 .02 / \mathrm{SR} 04$

$09.01 .03 .02 /$ SR12 $11.02 /$ SR02

\#E 064 <set polymarker representation>:

$04.02 .02 .02 /$ SR10 $04.02 .02 .02 /$ SR11 $04.02 .02 .02 /$ SR1 8 $04.02 .02 .02 /$ SR33 $04.02 .02 .02 /$ SR $34 \quad 04.02 .02 .02 /$ SR 35 $04.02 .02 .02 / S R 4104.02 .02 .02 / S R 42 \quad 04.02 .02 .02 / S R 43$ $04.02 .02 .02 /$ SR4 $404.02 .02 .02 /$ SR $45 \quad 04.02 .02 .02 /$ SR 46 $04.02 .02 .02 /$ SR $4704.02 .02 .02 /$ SR $48 \quad 05.02 /$ SR $1905.02 /$ SR2 0 $05.02 /$ SR21 09.01.01.01/SR03 09.01.01.02/SR03 09.01.02.01/SR04 $09.01 .02 .01 /$ SR09 $09.01 .02 .02 /$ SR04 09.01.02.02/SR0 9 09.01.03.01/SR01. 09.01.03.01/SR03 09.01.03.01/SR05 $09.01 .03 .01 / \mathrm{SR} 1209.01 .03 .02 / \mathrm{SR} 0109.01 .03 .02 / \mathrm{SR} 03$ $09.01 .03 .02 /$ SR0 $509.01 .03 .02 /$ SR12 $11.02 /$ SR0 2

\#E 065 <set text representation>: $04.02 .03 .02 /$ SR10 $04.02 .03 .02 /$ SR11 $04.02 .03 .02 /$ SR32. $04.02 .03 .02 /$ SR33 $04.02 .03 .02 /$ SR35 $04.02 .03 .02 /$ SR36 $04.02 .03 .02 /$ SR39 $04.02 .03 .02 /$ SR 40 $04.02 .03 .02 /$ SR5 $304.02 .03 .02 /$ SR55 $04.02 .03 .02 /$ SR 57 $04.02 .03 .02 /$ SR63 $04.02 .04 .02 / \mathrm{SR} 11$ $04.02 .04 .02 /$ SR20 $04.02 .04 .02 /$ SR2 9 $09.01 .01 .01 / \mathrm{SR} 03$ $09.01 .02 .01 /$ SR09 $09.01 .03 .01 /$ SR01 $09.01 .03 .01 /$ SR 12 $09.01 .03 .02 /$ SR0 6 $09.01 .10 .02 /$ SR01
$04.02 .03 .02 /$ SR 60 $04.02 .03 .02 /$ SR 64 $04.02 .04 .02 /$ SR 12 $04.02 .04 .02 / S R 24$ $05.02 /$ SR19 $05.02 /$ $09.01 .01 .02 / \mathrm{SR} 03$ $09.01 .02 .02 / \mathrm{SR} 04$ $09.01 .03 .01 / \mathrm{SR} 03$ $09.01 .03 .02 / S R 01$ $09.01 .03 .02 / \mathrm{SR} 12$ $09.01 .11 .01 /$ SR0 4
$04.02 .03 .02 / S R 18$ $04.02 .03 .02 /$ SR3 4 $04.02 .03 .02 /$ SR 38 $04.02 .03 .02 / S R 47$ $04.02 .03 .02 /$ SR 56 $04.02 .03 .02 /$ SR 61 $04.02 .04 .02 /$ SR10 $04.02 .04 .02 /$ SR13 $04.02 .04 .02 / S R 27$ /SR20 $05.02 /$ SR2 1 $09.01 .02 .01 / \mathrm{SRO} 4$ $09.01 .02 .02 /$ SR0 9 $09.01 .03 .01 /$ SR0 6 $09.01 .03 .02 /$ SR0 3 $09.01 .10 .01 / \mathrm{SR} 01$ $09.01 .11 .02 /$ SR01 $11.02 / S R 02$

\#F 066 <set interior representation>: $04.02 .05 .02 /$ SR11 $04.02 .05 .02 /$ SR12 $04.02 .05 .02 /$ SR19 $04.02 .05 .02 /$ SR31 $04.02 .05 .02 /$ SR33 $04.02 .05 .02 /$ SR 35 $04.02 .05 .02 /$ SR $3704.02 .05 .02 /$ SR $3804.02 .05 .02 /$ SR 39 
$04.02 .05 .02 / S R 40 \quad 04.02 .05 .02 / S R 4104.02 .05 .02 / S R 50$ $04.02 .05 .02 /$ SR5 1 $09.01 .01 .01 /$ SR03 $09.01 .02 .01 /$ SR0 9 $09.01 .03 .01 /$ SR01 $09.01 .03 .01 /$ SR11 $09.01 .03 .02 / \mathrm{SR} 03$ $09.01 .03 .02 /$ SR12 $09.01 .11 .01 / \mathrm{SR} 04$

$05.02 / S R 1905.02 / S R 20 \quad 05.02 / S R 21$

$09.01 .01 .02 /$ SR03 09.01.02.01/SR0 4 $09.01 .02 .02 /$ SR0 $409.01 .02 .02 /$ SR0 9 $09.01 .03 .01 /$ SR03 $09.01 .03 .01 /$ SR0 8 $09.01 .03 .01 /$ SR12 $09.01 .03 .02 /$ SR01 $09.01 .03 .02 /$ SR0 $809.01 .03 .02 /$ SR11 $09.01 .10 .01 /$ SR01 $09.01 .10 .02 /$ SR01 $09.01 .11 .02 /$ SR0 $111.02 /$ SR02

\#F 067 <set edge representation>: $04.02 .05 .03 /$ SR10 $04.02 .05 .03 /$ SR11 $04.02 .05 .03 /$ SR2 $904.02 .05 .03 /$ SR30 $04.02 .05 .03 /$ SR3 7 $04.02 .05 .03 / S R 45$ $04.02 .05 .03 /$ SR4 $8 \quad 04.02 .05 .03 /$ SR 49 05.02/SR20 05.02/SR21 09.01.01.01/SR03 $09.01 .01 .02 /$ $05.02 / \mathrm{SR} 2005.02 / \mathrm{SR} 2109.01 .01 .01 / \mathrm{SR} 0309.01 .01 .02 / \mathrm{SR} 03$
$09.01 .02 .01 / \mathrm{SR} 0409.01 .02 .01 / \mathrm{SR} 0909.01 .02 .02 / \mathrm{SR} 04$ $09.01 .02 .02 /$ SR0 9 $09.01 .03 .01 /$ SR0 7 $09.01 .03 .02 /$ SR0 3 $09.01 .10 .01 / \mathrm{SR} 01$ $09.01 .11 .02 /$ SR01

$04.02 .05 .03 /$ SR3 8 $04.02 .05 .03 /$ SR 46 $04.02 .05 .03 / S R 18$ $04.02 .05 .03 /$ SR 36 $04.02 .05 .03 /$ SR 44 $04.02 .05 .03 / S R 47$ $04.02 .05 .03 /$ SR50 $05.02 /$ SR19

$09.01 .03 .01 /$ SR01 $09.01 .03 .01 /$ SR12 $09.01 .03 .02 /$ SR0 7 $09.01 .03 .01 / \mathrm{SR} 03$ $09.01 .03 .02 /$ SR01 $09.01 .03 .02 /$ SR12 $09.01 .10 .02 /$ SR01 09.01.11.01/SR0 4 $11.02 / S R 02$

\#F 068 <set pattern representation>: $04.02 .05 .01 /$ SR75 $04.02 .05 .01 /$ SR76 $04.02 .05 .01 /$ SR77 $04.02 .05 .04 /$ SR01 $04.02 .05 .04 /$ SR02 $04.02 .05 .04 / S R 10$ $04.02 .05 .04 /$ SR19 $04.02 .05 .04 /$ SR20 $04.02 .05 .04 / S R 2105.02 / S R 19$ $05.02 / S R 2005.02 / S R 2109.01 .01 .01 / S R 0309.01 .01 .02 / S R 03$ $09.01 .02 .01 /$ SR0 $409.01 .02 .01 /$ SR09 $09.01 .02 .02 /$ SR0 4 $09.01 .02 .02 /$ SR09 $09.01 .03 .01 /$ SR03 09.01.03.01/SR11 $09.01 .03 .01 /$ SR12 $09.01 .03 .01 /$ SR15 $09.01 .03 .02 /$ SR03 $09.01 .03 .02 /$ SR11 $09.01 .03 .02 /$ SR12 $09.01 .03 .02 /$ SRI 5 $09.01 .10 .01 /$ SR03 09.01.10.02/SR03 11.02/SR02

\#F 069 <set colour representation>: $04.03 .02 .01 /$ SR01 $04.03 .02 .01 /$ SR02 $04.03 .02 .01 /$ SR0 8 $04.03 .02 .02 /$ SR0 $304.03 .02 .02 /$ SR0 $5 \quad 04.03 .02 .02 /$ SR0 6 $04.03 .02 .02 /$ SR2 $104.03 .02 .02 /$ SR2 $604.03 .02 .02 /$ SR2 7 $04.03 .02 .02 /$ SR30 $04.03 .02 .02 /$ SR31 $04.03 .02 .02 /$ SR33 $05.02 /$ SR 19 $05.02 / S R 2005.02 / S R 2109.01 .01 .01 /$ SR03 09.01.01.02/SR03 $09.01 .02 .01 /$ SR0 $409.01 .02 .01 /$ SR09 $09.01 .02 .02 /$ SR0 4 $09.01 .02 .02 /$ SR09 $09.01 .03 .01 /$ SR03 09.01.03.01/SR12 $09.01 .03 .01 /$ SR17 $09.01 .03 .02 /$ SR03 $09.01 .03 .02 /$ SR 12 $09.01 .03 .02 /$ SR17 $11.02 /$ SR02

\#F 070 <set highlighting filter>: $04.03 .04 .02 /$ SR01 $04.03 .04 .02 /$ SRO 4 $04.03 .04 .02 /$ SR11 $04.03 .04 .02 /$ SR12 $04.03 .04 .02 / S R 13 \quad 05.02 / S R 19$ $05.02 /$ SR20 05.02/SR21 09.01.01.01/SR03 09.01.01.02/SR03 $09.01 .02 .01 /$ SR0 $409.01 .02 .01 /$ SR09 $09.01 .02 .02 / S R 04$ $09.01 .02 .02 /$ SR09 09.01.10.01/SR03 09.01.10.02/SR03 11.02/SR02

\#F 071 <set invisibility filter>: 
$04.03 .04 .02 /$ SR05 $04.03 .04 .02 /$ SR0 $804.03 .04 .02 /$ SR0 9 $04.03 .04 .02 /$ SR10 $04.03 .04 .02 /$ SR11 $04.03 .04 .02 /$ SR 12 $04.03 .04 .02 /$ SR13 $05.02 /$ SR19 $05.02 / S R 2005.02 / S R 21$ $09.01 .01 .01 /$ SR03 09.01.01.02/SR03 09.01.02.01/SR0 4 09.01.02.01/SR09 09.01.02.02/SR04 09.01.02.02/SR09 09.01.10.01/SR03 09.01.10.02/SR03 11.02/SR02

\#F 072 <set colour model>:

$04.03 .02 .01 /$ SR05 $04.03 .02 .01 /$ SR09 $04.03 .02 .02 /$ SR 17

$04.03 .02 .02 / S R 2104.03 .02 .02 / S R 3209.01 .01 .01 / S R 03$

$09.01 .01 .02 /$ SR03 $09.01 .02 .01 /$ SR0 $409.01 .02 .01 /$ SR0 9

$09.01 .02 .02 /$ SR0 $409.01 .02 .02 /$ SR09 $09.01 .03 .01 /$ SR0 9

$09.01 .03 .02 /$ SR09 09.01.10.01/SR01 09.01.10.02/SR01

09.01.11.01/SR04 09.01.11.02/SR01 11.02/SR02

\#F 073 <set hlhsr identifier>:

$04.03 .03 / S R 0105.02 / S R 1905.02 / S R 20 \quad 05.02 / S R 2109.01 .01 .01 / S R 05$ $09.01 .01 .02 /$ SR05 $11.02 /$ SR02

\#F 074 <set hlhsr mode>:

$04.03 .03 /$ SR0 $404.03 .03 /$ SR0 $604.03 .03 /$ SR0 $0404.03 .03 /$ SR1 1

$05.02 / S R 1905.02 / S R 2005.02 / S R 2109.01 .01 .01 / S R 03$

$09.01 .01 .02 /$ SR03 09.01.02.01/SR04 09.01.02.01/SR0 9

$09.01 .02 .02 /$ SR0 $409.01 .02 .02 /$ SR09 $09.01 .03 .01 / S R 10$

$09.01 .03 .02 /$ SR10 $11.02 /$ SR02

\#F 075 <set local transformation 3>:

$02.03 .03 / S R 10 \quad 05.02 / S R 19 \quad 05.02 / S R 20 \quad 05.02 / S R 21 \quad 06.01 .02 / S R 01$

$06.01 .02 /$ SR07 $06.01 .02 /$ SR0 8 06.01.02/SR16 $06.01 .02 /$ SR17

09.01.01.01/SR05 09.01.01.02/SR05 09.01.10.01/SR01

09.01.10.02/SR01 09.01.11.01/SR04 09.01.11.02/SR01 $11.02 / S R 02$

\#F 076 <set local transformation>:

$02.03 .03 /$ SR10 $05.02 /$ SR19 $05.02 /$ SR20 $05.02 / S R 21 \quad 06.01 .02 / S R 04$

$06.01 .02 /$ SR0 $706.01 .02 / S R 16 \quad 06.01 .02 / S R 1706.01 .02 / S R 18$

09.01.01.01/SR05 09.01.01.02/SR05 09.01.10.01/SR01

09.01.10.02/SR01 09.01.11.01/SR04 09.01.11.02/SR01 $11.02 /$ SR02

\#F 077 <set global transformation 3>:

$02.03 .03 /$ SR10 $05.02 /$ SR19 $05.02 /$ SR20 $05.02 /$ SR21 $06.01 .02 /$ SR0 9

06.01.02/SR15 06.01.02/SR16 $06.01 .02 /$ SR17 $09.01 .01 .01 /$ SR0 5

$09.01 .01 .02 /$ SR05 $11.02 /$ SR02

\#F 078 <set global transformation>:

$02.03 .03 / S R 10 \quad 05.02 / S R 1905.02 / S R 20 \quad 05.02 / S R 2106.01 .02 / S R 12$

$06.01 .02 / S R 1606.01 .02 / S R 1706.01 .02 / S R 18 \quad 09.01 .01 .01 /$ SR0 5

$09.01 .01 .02 /$ SR05 $11.02 /$ SR02

\#F 079 <set modelling clipping volume $3>$ :

$02.03 .03 / S R 1005.02 / S R 1905.02 / S R 20 \quad 05.02 / S R 2106.01 .02 / S R 23$

$06.01 .02 /$ SR3 $606.01 .02 /$ SR37 $06.01 .02 /$ SR3 $806.01 .02 /$ SR3 9

$06.01 .02 / S R 40 \quad 06.01 .02 / S R 4106.01 .02 / S R 42 \quad 06.01 .02 / S R 43$

$06.01 .02 /$ SR44 $06.01 .02 /$ SR45 $06.01 .02 / S R 46 \quad 06.01 .02 / S R 47$

$06.01 .02 /$ SR48 $06.01 .02 /$ SR57 09.01.01.01/SR05 09.01.01.02/SR05 
09.01.10.01/SR03 09.01.10.02/SR03 11.02/SR02

\#F 080 <set modelling clipping volume>:

$02.03 .03 /$ SR10 05.02/SR19 05.02/SR20 05.02/SR21 $06.01 .02 / S R 26$

$06.01 .02 /$ SR3 $66.01 .02 /$ SR37 $06.01 .02 /$ SR38 $06.01 .02 /$ SR39

06.01.02/SR49 06.01.02/SR57 09.01.01.01/SR05 09.01.01.02/SR05 09.01.10.01/SR03 09.01.10.02/SR03 11.02/SR02

\#F 081 <set modelling clipping indicator>:

05.02/SR19 05.02/SR20 05.02/SR21 06.01.02/SR29 06.01.02/SR52

06.01.02/SR53 06.01.02/SR58 09.01.01.01/SR05 09.01.01.02/SR05 09.01.10.01/SR01 09.01.10.02/SR01 09.01.11.01/SR04

$09.01 .11 .02 /$ SR01 $11.02 /$ SR02

\#F 082 <restore modelling clipping volume>:

02.03.03/SR10 $05.02 /$ SR19 $05.02 /$ SR20 $05.02 /$ SR2 $106.01 .02 /$ SR 32

06.01.02/SR5 $66.01 .02 /$ SR5 $09.01 .01 .01 /$ SR0 5 09.01.01.02/SR0 5 $11.02 /$ SR0 2

\#F 083 <set view index>:

05.02/SR10 05.02/SR19 05.02/SR20 05.02/SR21 06.02.02/SR01

$09.01 .01 .01 /$ SR0 $509.01 .01 .02 /$ SR0 $509.01 .03 .01 /$ SR13

$09.01 .03 .02 /$ SR13 $11.02 /$ SR02

\#F 084 <set view representation $3>$ :

05.02 /SR10 05.02/SR19 05.02/SR20 05.02/SR21 $06.02 .02 /$ SR1 4

06.02.02/SR15 06.02.02/SR23 09.01.01.01/SR03 09.01.01.02/SR03

$09.01 .02 .01 /$ SR0 4 09.01.02.01/SR0 $09.01 .02 .02 /$ SR0 4

09.01.02.02/SR07 09.01.03.01/SR14 09.01.03.02/SR14

$09.01 .04 .01 /$ SR01 09.01.04.01/SR04 09.01.04.01/SR05

09.01 .04 .02 /SR01 09.01.04.02/SR04 09.01.04.02/SR05

$09.01 .10 .01 /$ SR01 $09.01 .10 .02 /$ SR01 09.01.11.01/SR0 4

$09.01 .11 .02 /$ SR01 $11.02 /$ SR02

\#F 085 <set view representation>:

05.02/SR10 05.02/SR19 05.02/SR20 $05.02 /$ SR21 $06.02 .02 /$ SR1 4

$06.02 .02 /$ SR15 06.02.02/SR16 06.02.02/SR23 09.01.01.01/SR03

$09.01 .01 .02 /$ SR03 09.01.02.01/SR04 09.01.02.01/SR07

09.01 .02 .02 /SR0 4 09.01.02.02/SR07 09.01.03.01/SR14

$09.01 .03 .02 /$ SR14 09.01.04.01/SR01 09.01.04.01/SR0 4

$09.01 .04 .01 /$ SR05 09.01.04.02/SR01 09.01.04.02/SR04

$09.01 .04 .02 /$ SR0 $509.01 .10 .01 /$ SR01 $09.01 .10 .02 /$ SR01

$09.01 .11 .01 /$ SR0 $409.01 .11 .02 /$ SR01 $11.02 /$ SR02

\#F 086 <set view transformation input priority>:

06.02.02/SR21 06.02.02/SR22 09.01.01.01/SR03 09.01.01.02/SR03

$09.01 .02 .01 /$ SR0 409.01 .02 .01 /SR0 $09.01 .02 .02 /$ SR0 4

$09.01 .02 .02 /$ SR07 09.01.03.01/SR02 09.01.03.01/SR13

09.01.03.02/SR02 09.01.03.02/SR13 09.01.10.01/SR01

09.01.10.02/SR01 09.01.11.01/SR04 09.01.11.02/SR01 11.02/SR02

\#F 087 <set workstation window $3>$ :

05.02 /SR10 05.02/SR19 05.02/SR20 05.02/SR21 06.03/SR06 06.03/SR0 8

06.03/SR14 09.01.01.01/SR03 09.01.01.02/SR03 09.01.02.01/SR0 4 
09.01.02.01/SR07 09.01.02.02/SR04 09.01.02.02/SR07

$09.01 .04 .01 /$ SR02 $09.01 .04 .01 /$ SR0 $609.01 .04 .02 /$ SR0 2

$09.01 .04 .02 /$ SR0 $611.02 /$ SR02

\#F 088 <set workstation window>:

$05.02 / S R 10 \quad 05.02 / S R 1905.02 / S R 20 \quad 05.02 / S R 21 \quad 06.03 / S R 07 \quad 06.03 / S R 08$

$06.03 /$ SR14 09.01.01.01/SR03 09.01.01.02/SR03 09.01.02.01/SR04

$09.01 .02 .01 /$ SR0 $709.01 .02 .02 /$ SR04 09.01.02.02/SR0 7

$09.01 .04 .01 /$ SR02 $09.01 .04 .01 /$ SR06 $09.01 .04 .02 /$ SR02

$09.01 .04 .02 /$ SR0 $611.02 /$ SR02

\#F 089 <set workstation viewport 3>:

05.02/SR10 05.02/SR19 $05.02 / S R 2005.02 / S R 2106.03 /$ SR09 $06.03 /$ SR11

$06.03 /$ SRI4 09.01.01.01/SR03 09.01.01.02/SR03 09.01.02.01/SR0 4

$09.01 .02 .01 /$ SR0 $09.01 .02 .02 /$ SR0 $409.01 .02 .02 /$ SR0 7

$09.01 .04 .01 /$ SR03 $09.01 .04 .01 /$ SR0 $09.01 .04 .02 /$ SR03

$09.01 .04 .02 /$ SR0 $711.02 / S R 02$

\#F 090 <set workstation viewport>:

$05.02 /$ SR10 $05.02 / S R 1905.02 / S R 20 \quad 05.02 / S R 2106.03 / S R 1006.03 / S R 11$

06.03/SR14 09.01.01.01/SR03 09.01.01.02/SR03 09.01.02.01/SR04

$09.01 .02 .01 /$ SR0 $709.01 .02 .02 /$ SR0 $409.01 .02 .02 /$ SR0 7

$09.01 .04 .01 /$ SR03 09.01.04.01/SR07 09.01.04.02/SR03

$09.01 .04 .02 /$ SR0 $711.02 /$ SR02

\#F 091 <translate 3>:

06.01.01/SR01 06.01.01/SR02 06.01.01/SR03 09.02.01/SR01 $11.02 /$ SR02

\#F $092<$ <ranslate>:

06.01.01/SR01 06.01.01/SR02 06.01.01/SR04 09.02.01/SR01 $11.02 /$ SR02

\#F $093<$ scale $3>$ :

06.01.01/SR01 06.01.01/SR02 06.01.01/SR05 09.02.01/SR01 $11.02 / \mathrm{SRO} 2$

\#F $094<$ scale>:

06.01.01/SR01 06.01.01/SR02 06.01.01/SR06 09.02.01/SR01 $11.02 /$ SR02

\#F 095 <rotate $x>$ :

06.01.01/SR01 06.01.01/SR02 06.01.01/SR07 09.02.01/SR01 $11.02 / \mathrm{SRO} 2$

\#F 096 <rotate $y>$ :

06.01.01/SR01 $06.01 .01 /$ SR02 $06.01 .01 /$ SR08 $09.02 .01 /$ SR01 $11.02 / \mathrm{SR} 02$

\#F 097 <rotate $z>$ :

06.01.01/SR01 06.01.01/SR02 06.01.01/SR09 09.02.01/SR01 $11.02 /$ SR0 2

\#F $098<$ rotate> 
06.01.01/SR01 06.01.01/SR02 $06.01 .01 / \mathrm{SR} 10 \quad 09.02 .01 / \mathrm{SR} 01$ $11.02 / \mathrm{SR} 02$

\#F 099 <compose matrix 3>:

06.01.01/SR01 06.01.01/SR02 06.01.01/SR11 09.02.01/SR01 $11.02 /$ SR0 2

\#F 100 <compose matrix>:

06.01.01/SR01 06.01.01/SR02 06.01.01/SR12 09.02.01/SR01 $11.02 /$ SR02

\#F 101 <transform point 3>:

06.01.01/SR01 06.01.01/SR02 06.01.01/SR13 09.02.01/SR01 $11.02 /$ SR02

\#F 102 <transform point>:

06.01.01/SR01 06.01.01/SR02 $06.01 .01 /$ SR14 09.02.01/SR01 $11.02 / \mathrm{SR} 02$

\#F 103 <build transformation matrix 3>:

06.01.01/SR01 06.01.01/SR02 06.01.01/SR15 09.02.01/SR01 $11.02 / \mathrm{SR} 02$

\#F 104 <build transformation matrix>:

06.01.01/SR01 06.01.01/SR02 06.01.01/SR16 09.02.01/SR01 $11.02 /$ SR02

\#F 105 <compose transformation matrix 3>:

06.01.01/SR01 06.01.01/SR02 06.01.01/SR17 09.02.01/SR01 $11.02 / \mathrm{SR} 02$

\#F 106 <compose transformation matrix>:

06.01.01/SR01 06.01.01/SR02 06.01.01/SR18 09.02.01/SR01 $11.02 /$ SR02

\#F 107 <evaluate view orientation matrix 3>:

06.02.01/SR01 06.02.01/SR02 06.02.01/SR03 06.02.01/SR0 4 $09.02 .01 /$ SR01 $09.02 .04 /$ SR05 $09.02 .04 /$ SR0 $609.02 .04 /$ SR0 7 11.02 /SR02

\#F 108 <evaluate view orientation matrix>:

$06.02 .01 /$ SR01 06.02.01/SR02 06.02.01/SR05 $06.02 .01 /$ SR0 6 $09.02 .01 /$ SR01 09.02.04/SR06 11.02/SR02

\#F 109 <evaluate view mapping matrix 3>:

$06.02 .01 /$ SR01 $06.02 .01 /$ SR02 $06.02 .01 /$ SR07 $06.02 .01 /$ SR0 8 $06.02 .01 /$ SR09 $06.02 .01 /$ SR10 $09.02 .01 /$ SR0 $1 \quad 09.02 .04 /$ SR0 1 $09.02 .04 /$ SR02 $09.02 .04 /$ SR03 $09.02 .04 /$ SR0 $409.02 .04 /$ SR0 8 $09.02 .04 /$ SR09 $09.02 .04 /$ SR10 $11.02 /$ SR02

\#F 110 <evaluate view mapping matrix>: $06.02 .01 /$ SR01 $06.02 .01 /$ SR02 $06.02 .01 /$ SR11 $06.02 .01 /$ SR12 $06.02 .01 /$ SR13 09.02.01/SR01 09.02.04/SR01 09.02.04/SR02 $09.02 .04 / \mathrm{SR} 0311.02 / \mathrm{SR} 02$ 
\#F 111 <open structure>:

02.01.01/SR01 02.01.01/SR09 02.01.01/SR10 02.02.01/SR01

$02.02 .01 /$ SR02 $02.02 .01 /$ SR03 $05.03 /$ SR10 09.01.01.01/SR0 6

$09.01 .01 .02 /$ SR0 $611.02 /$ SR02

\#F 112 <close structure>:

02.02.01/SR04 09.01.01.01/SR05 09.01.01.02/SR05 11.02/SR02

\#F 113 <execute structure>:

02.01/SR01 02.01/SR05 02.01/SR06 02.01.01/SR03 02.01.01/SR09

02.01.01/SR10 02.03.03/SR02 03.04/SR07 03.04/SR0 $03.04 / S R 09$

$04.02 .01 .01 /$ SR12. $04.02 .01 .01 /$ SR18 $04.02 .01 .01 /$ SR2 4

$04.02 .01 .02 /$ SR0 $904.02 .02 .01 /$ SR12 $04.02 .02 .01 /$ SR1 9

$04.02 .02 .01 /$ SR25 $04.02 .02 .02 /$ SR0 $904.02 .03 .01 /$ SR2 4

$04.02 .03 .01 /$ SR33 $04.02 .03 .01 /$ SR39 $04.02 .03 .01 / S R 45$

$04.02 .03 .01 /$ SR51 $04.02 .03 .01 /$ SR63 $04.02 .03 .01 /$ SR7 1

$04.02 .03 .01 /$ SR78 $04.02 .03 .01 /$ SR83 $04.02 .03 .02 /$ SR0 9

$04.02 .04 .01 / \mathrm{SR} 1704.02 .04 .01 / \mathrm{SR} 2504.02 .04 .01 / \mathrm{SR} 31$

$04.02 .04 .01 /$ SR36 $04.02 .04 .01 /$ SR $4104.02 .04 .01 /$ SR5 4

$04.02 .04 .01 /$ SR62 $04.02 .04 .01 /$ SR69 $04.02 .04 .01 /$ SR7 4

$04.02 .04 .01 /$ SR8 $404.02 .04 .02 /$ SR0 $704.02 .05 .01 /$ SR 17

$04.02 .05 .01 /$ SR25 $04.02 .05 .01 /$ SR $3104.02 .05 .01 /$ SR 39

$04.02 .05 .01 /$ SR $4804.02 .05 .01 /$ SR5 $404.02 .05 .01 /$ SR 60

$04.02 .05 .01 / \operatorname{SR} 6504.02 .05 .01 /$ SR81 $04.02 .05 .01 /$ SR83

$04.02 .05 .02 /$ SR10 $04.02 .05 .03 /$ SR09 $04.03 .01 /$ SR11 $04.03 .04 .01 /$ SR 14

$05.02 / S R 1905.02 / S R 20 \quad 05.02 / S R 2105.03 / S R 1006.01 .02 / S R 20$

$06.01 .02 / S R 2106.01 .02 / S R 2206.01 .02 / S R 5106.01 .02 / S R 55$

$09.01 .01 .01 /$ SR0 $509.01 .01 .02 /$ SR05 $11.02 /$ SR02

\#F $114<$ label>:

02.02.02/SR01 09.01.01.01/SR05 09.01.01.02/SR05 11.02/SR02

\#F 115 <application data>:

02.02.02/SR04 09.01.01.01/SR05 09.01.01.02/SR05 11.02/SR02

\#F 116 <generalized structure element>:

$02.02 .02 /$ SR0 $702.02 .02 /$ SR0 $802.02 .02 / S R 1105.02 / S R 1905.02 / S R 20$

05.02/SR21 09.01.01.01/SR05 09.01.01.02/SR05 11.02/SR02

\#F $117<$ set edit mode> :

$02.02 .03 /$ SR01 $02.02 .03 /$ SR02 $02.02 .03 /$ SR03 $02.02 .03 /$ SR0 4

$02.02 .03 /$ SR0 $502.02 .03 /$ SR0 $609.01 .01 .01 /$ SR02 $09.01 .01 .02 / S R 02$

$09.01 .10 .01 /$ SR01 09.01.10.02/SR01 09.01.11.01/SR0 4

$09.01 .11 .02 /$ SR01 $11.02 /$ SR02

\#F 118 <Copy al1 elements from structure>:

$02.02 .05 /$ SR01 $02.02 .05 /$ SR02 $02.02 .05 /$ SR03 $02.02 .05 /$ SR0 4

05.02/SR19 05.02/SR20 05.02/SR21 09.01.01.01/SR05

$09.01 .01 .02 /$ SR05 $11.02 /$ SR02

\#F 119 <set element pointer>:

02.02.03/SR07 02.02.03/SR10 09.01.01.01/SR05 09.01.01.02/SR05

$11.02 / \mathrm{SR} 02$ 
\#F 120 <offset element pointer>:

02.02.03/SR08 02.02.03/SR10 09.01.01.01/SR05 09.01.01.02/SR05 $11.02 / \mathrm{SR} 02$

\#F 121 <set element pointer at label>:

02.02.03/SR09 09.01.01.01/SR05 09.01.01.02/SR05 09.01.05.01/SR03 $09.01 .05 .02 /$ SR03 $11.02 /$ SR02

\#F 122 <delete element>:

02.02.04/SR01 02.02.04/SR02 02.02.04/SR07 05.02/SR19 05.02/SR20 $05.02 / S R 2109.01 .01 .01 /$ SR05 $09.01 .01 .02 /$ SR05 $11.02 /$ SR02

\#F 123 <delete element range>:

02.02.04/SR03 02.02.04/SR0 $402.02 .04 / S R 07 \quad 05.02 / S R 1905.02 / S R 20$ $05.02 / S R 2109.01 .01 .01 /$ SR0 $509.01 .01 .02 /$ SR0 $511.02 /$ SR02

\#F 124 <delete elements between labels>:

02.02.04/SR05 02.02.04/SR06 02.02.04/SR07 05.02/SR19 05.02/SR20 05.02/SR21 09.01.01.01/SR05 09.01.01.02/SR05 09.01.05.01/SR0 4 $09.01 .05 .02 / \mathrm{SR} 0411.02 / \mathrm{SR} 02$

\#E 125 <empty structure>:

$02.01 .01 /$ SR02 02.01.01/SR09 02.01.01/SR10 02.02.04/SR0 8

$02.02 .04 /$ SR0 $902.02 .04 /$ SR10 $02.02 .04 /$ SR11 $05.02 /$ SR19 $05.02 / S R 20$ $05.02 /$ SR21 $05.03 /$ SR10 09.01.01.01/SR02 09.01.01.02/SR02 $11.02 / \mathrm{SR} 02$

\#E 126 <delete structure>:

$02.01 .02 .01 /$ SR01 $02.01 .02 .01 /$ SR02 $02.01 .02 .01 / S R 03$

$02.01 .02 .01 / \mathrm{SR} 0402.01 .02 .01 / \mathrm{SR} 0502.01 .02 .01 / \mathrm{SR} 06$

$02.01 .02 .01 /$ SR0 $705.02 / S R 19 \quad 05.02 / S R 20 \quad 05.02 / S R 21$

$09.01 .01 .01 /$ SR02 09.01.01.02/SR02 11.02/SR02

\#F 127 <delete structure network>:

$02.01 .02 .02 /$ SR01 $02.01 .02 .02 /$ SR02 $02.01 .02 .02 /$ SR0 3

$02.01 .02 .02 / \mathrm{SR} 0402.01 .02 .02 / \mathrm{SR} 05 \quad 02.01 .02 .02 / \mathrm{SR} 06$

$02.01 .02 .02 /$ SR0 $702.01 .02 .02 /$ SR0 $802.01 .02 .02 /$ SR0 9

$02.01 .02 .02 /$ SR10 $02.01 .02 .02 /$ SR11 $02.01 .02 .02 /$ SR12 $05.02 / S R 19$

$05.02 /$ SR20 05.02/SR21 09.01.01.01/SR02 09.01.01.02/SR02

09.01.10.01/SR01 09.01.10.02/SR01 09.01.11.01/SR0 4

$09.01 .11 .02 /$ SR01 $11.02 /$ SR02

\#F 128 <delete all structures>:

$02.01 .02 .03 /$ SR01 $02.01 .02 .03 /$ SR02 $05.02 /$ SR19 $05.02 /$ SR20

$05.02 / S R 2109.01 .01 .01 /$ SR02 09.01.01.02/SR02 $11.02 /$ SR02

\#F 129 <change structure identifier>:

02.01.01/SR05 02.01.01/SR09 02.01.01/SR10 02.01.03.01/SR01

$02.01 .03 .01 /$ SR02 $02.01 .03 .01 /$ SR03 $02.01 .03 .01 / S R 04$

$02.01 .03 .01 /$ SR05 $02.01 .03 .01 /$ SR0 $605.02 /$ SR19 $05.02 /$ SR20

05.02/SR21 09.01.01.01/SR02 09.01.01.02/SR02 11.02/SR02

\#F 130 <change structure references>: 
02.01.01/SR06 02.01.01/SR09 02.01.01/SR10 02.01.03.02/SR01

$02.01 .03 .02 /$ SR0 $202.01 .03 .02 /$ SR03 $02.01 .03 .02 /$ SR0 4

$02.01 .03 .02 /$ SR0 $502.01 .03 .02 /$ SR0 $602.01 .03 .03 /$ SR0 7

$02.01 .03 .03 /$ SR0 $85.02 /$ SR19 $05.02 /$ SR20 $05.02 / S R 21$

09.01.01.01/SR02 09.01.01.02/SR02 11.02/SR02

\#F 131 <change structure identifier and references>:

02.01.01/SR07 02.01.01/SR09 02.01.01/SR10 02.01.03.03/SR01

$02.01 .03 .03 / \mathrm{SR} 0202.01 .03 .03 / \mathrm{SR} 0302.01 .03 .03 / \mathrm{SR} 04$

$02.01 .03 .03 /$ SR0 $502.01 .03 .03 /$ SR0 $602.01 .03 .03 /$ SR0 7

$02.01 .03 .03 /$ SR0 $805.02 / S R 1905.02 / S R 20 \quad 05.02 / S R 21$

09.01.01.01/SR02 09.01.01.02/SR02 11.02/SR02

\#F 132 <post structure>:

02.01.01/SR04 02.01.01/SR09 02.01.01/SR10 05.02/SR10 05.02/SR19

$05.02 / S R 2005.02 / S R 2105.03 / S R 0105.03 / S R 0205.03 / S R 0305.03 / S R 04$

$09.01 .01 .01 / \mathrm{SR} 0309.01 .01 .02 / \mathrm{SR} 03 \quad 09.01 .02 .01 / \mathrm{SR} 04$

$09.01 .02 .01 /$ SR09 $09.01 .02 .02 /$ SR04 $09.01 .02 .02 /$ SR0 9

$09.01 .05 .01 /$ SR0 $609.01 .05 .02 /$ SR0 $611.02 /$ SR02

\#F 133 <unpost structure>:

05.02/SR19 05.02/SR20 05.02/SR21 05.03/SR05 05.03/SR0 $05.03 / S R 07$ $09.01 .01 .01 /$ SR03 09.01.01.02/SR03 09.01.02.01/SR0 4

09.01.02.01/SR09 09.01.02.02/SR04 09.01.02.02/SR09 11.02/SR02

\#F 134 <unpost all structures>:

$05.02 / S R 1905.02 / S R 20 \quad 05.02 / S R 2105.03 / S R 08 \quad 05.03 /$ SR0 9

$09.01 .01 .01 /$ SR03 $09.01 .01 .02 /$ SR03 $09.01 .02 .01 /$ SR0 4

$09.01 .02 .01 /$ SR09 09.01.02.02/SR04 09.01.02.02/SR09 11.02/SR02

\#F 135 <open archive file>:

03.01/SR05 03.01/SR06 03.01/SR07 09.01.01.01/SR02

$09.01 .01 .02 /$ SR02 09.01.09.01/SR01 09.01.09.01/SR02

$09.01 .09 .01 /$ SR03 09.01.09.01/SR04 09.01.09.02/SR01

$09.01 .09 .02 / \mathrm{SR} 02$ 09.01.09.02/SR03 09.01.09.02/SR04 $11.02 / \mathrm{SR} 02$

\#F 136 <close archive file>:

03.01/SR08 03.01/SR09 09.01.01.01/SR07 09.01.01.02/SR07

09.01.09.01/SR05 09.01.09.02/SR05 11.02/SR02

\#F 137 <archive structures>:

03.01 /SR0 $703.03 /$ SR0 $103.03 /$ SR0 4 03.03/SR05 03.03/SR0 $603.03 /$ SR0

$03.03 /$ SR0 $809.01 .01 .01 /$ SR0 $09.01 .01 .02 / S R 0709.01 .05 .01 /$ SR01

09.01.05.02/SR01 09.01.09.01/SR05 09.01.09.01/SR06

$09.01 .09 .01 /$ SR07 $09.01 .09 .02 /$ SR0 5 09.01.09.02/SR0 6

09.01.09.02/SR07 09.01.10.01/SR03 09.01.10.02/SR03 11.02/SR02

\#F 138 <archive structure networks>:

03.01/SR07 03.03/SR02 03.03/SR04 03.03/SR05 03.03/SR06 03.03/SR07

$03.03 /$ SR0 8 09.01.01.01/SR07 09.01.01.02/SR07 09.01.05.01/SR01

$09.01 .05 .02 /$ SR01 $09.01 .09 .01 /$ SR0 $509.01 .09 .01 /$ SR0 6

$09.01 .09 .01 /$ SR07 $09.01 .09 .02 /$ SR0 $509.01 .09 .02 /$ SR0 6

$09.01 .09 .02 /$ SR07 09.01.10.01/SR03 09.01.10.02/SR03 $11.02 /$ SR02 
\#F 139 <archive all structures>:

$03.01 /$ SR0 $703.03 /$ SR0 $303.03 /$ SR0 $403.03 /$ SR0 $503.03 /$ SR0 $603.03 /$ SR0 07 03.03/SR08 09.01.01.01/SR07 09.01.01.02/SR07 09.01.09.01/SR05 09.01.09.01/SR06 09.01.09.01/SR07 09.01.09.02/SR05

$09.01 .09 .02 /$ SR06 09.01.09.02/SR07 11.02/SR02

\#F 140 <set conflict resolution>:

03.02/SR02 09.01.01.01/SR02 09.01.01.02/SR02 09.01.10.01/SR01

09.01.10.02/SR01 09.01.11.01/SR04 09.01.11.02/SR01 $11.02 / \mathrm{SR} 02$

\#F 141 <retrieve structure identifiers>:

03.01/SR07 03.04/SR01 09.01.01.01/SR07 09.01.01.02/SR07

09.01.09.01/SR05 09.01.09.02/SR05 09.01.10.01/SR02

$09.01 .10 .02 /$ SR02 $11.02 /$ SR02

\#F 142 <retrieve structures>:

03.01/SR07 03.03/SR09 03.03/SR12 03.03/SR13 03.03/SR14 03.03/SR15 $03.03 /$ SR1 $603.03 /$ SR17 $03.03 /$ SR18 $03.03 /$ SR1 $905.02 / S R 1905.02 / S R 20$ 05.02/SR21 09.01.01.01/SR07 09.01.01.02/SR07 09.01.09.01/SR05 09.01.09.01/SR06 09.01.09.01/SR09 09.01.09.02/SR05 $09.01 .09 .02 /$ SR0 $699.01 .09 .02 /$ SR09 $09.01 .10 .01 /$ SR03 $09.01 .10 .02 /$ SR03 $11.02 /$ SR02

\#F 143 <retrieve structure networks>:

03.01/SR07 03.03/SR10 03.03/SR12 $03.03 / S R 1303.03 /$ SR14 $03.03 /$ SR15 $03.03 /$ SR1 $603.03 /$ SR17 $03.03 /$ SR18 $03.03 /$ SR19 $05.02 /$ SR19 $05.02 / S R 20$ 05.02/SR21 09.01.01.01/SR07 09.01.01.02/SR07 09.01.09.01/SR05 09.01.09.01/SR06 09.01.09.01/SR09 09.01.09.02/SR05 $09.01 .09 .02 /$ SR06 09.01.09.02/SR09 09.01.10.01/SR03 $09.01 .10 .02 /$ SR03 $11.02 /$ SR02

\#F 144 <retrieve all structures>:

03.01/SR07 03.03/SR11 03.03/SR12 $03.03 /$ SR13 $03.03 /$ SR14 $03.03 /$ SR15 $03.03 /$ SR1 $603.03 /$ SR17 $03.03 /$ SR18 $03.03 /$ SR19 $05.02 /$ SR19 $05.02 / S R 20$ 05.02/SR21 09.01.01.01/SR07 09.01.01.02/SR07 09.01.09.01/SR05 09.01.09.01/SR06 09.01.09.02/SR05 09.01.09.02/SR06 11.02/SR02

\#F 145 <retrieve paths to ancestors>:

03.01/SR0 $703.04 /$ SR0 $503.04 /$ SR0 $03.04 /$ SR0 $803.04 /$ SR10 $03.04 / S R 12$ $03.04 /$ SR13 03.04/SR16 09.01.01.01/SR07 09.01.01.02/SR07

09.01.05.01/SR02 09.01.05.01/SR05 09.01.05.02/SR02 09.01.05.02/SR05 09.01.10.01/SR01 09.01.10.01/SR02 $09.01 .10 .02 /$ SR01 09.01.10.02/SR02 09.01.11.01/SR0 4 $09.01 .11 .02 /$ SR01 $11.02 /$ SR02

\#F 146 <retrieve paths to descendants>:

03.01/SR07 03.04/SR06 03.04/SR07 03.04/SR08 03.04/SR09 03.04/SR11 $03.04 /$ SR14 03.04/SR15 03.04/SR17 09.01.01.01/SR07

$09.01 .01 .02 /$ SR07 09.01.05.01/SR02 09.01.05.01/SR05

$09.01 .05 .02 /$ SR02 $09.01 .05 .02 /$ SR05 $09.01 .10 .01 /$ SR01

09.01.10.01/SR02 09.01.10.02/SR01 09.01.10.02/SR02

$09.01 .11 .01 /$ SR04 09.01.11.02/SR01 11.02/SR02

\#F 147 <delete structures from archive>: 
03.01/SR07 03.05/SR01 03.05/SR04 03.05/SR05 09.01.01.01/SR07 09.01.01.02/SR07 09.01.09.01/SR05 09.01.09.01/SR08 09.01.09.02/SR05 09.01.09.02/SR08 09.01.10.01/SR03 $09.01 .10 .02 /$ SR03 $11.02 /$ SR02

\#F 148 <delete structure networks from archive>: 03.01/SR07 03.05/SR02 03.05/SR04 03.05/SR05 09.01.01.01/SR07 $09.01 .01 .02 /$ SR07 09.01.09.01/SR05 09.01.09.01/SR08 09.01.09.02/SR05 09.01.09.02/SR08 09.01.10.01/SR03 $09.01 .10 .02 /$ SR03 $11.02 /$ SR02

\#F 149 <delete all structures from archive>:

03.01/SR0 $703.05 /$ SR03 03.05/SR0 4 03.05/SR05 09.01.01.01/SR07 09.01.01.02/SR07 09.01.09.01/SR05 09.01.09.02/SR05 $11.02 /$ SR02

\#F 150 <set pick identifier>:

$05.02 /$ SR19 05.02/SR20 05.02/SR21 09.01.01.01/SR0 5 $09.01 .01 .02 /$ SR0 $511.02 /$ SR02

\#F 151 <set pick filter>: 09.01.01.01/SR03 09.01.01.02/SR03 09.01.02.01/SR0 4 $09.01 .02 .01 /$ SR10 $09.01 .02 .02 /$ SR0 4 09.01.02.02/SR10 $09.01 .10 .01 /$ SR03 $09.01 .10 .02 /$ SR03 $11.02 /$ SR02

\#F 152 <initialize locator 3>:

09.01.01.01/SR03 09.01.01.02/SR03 09.01.02.01/SR0 4 $09.01 .02 .01 /$ SR11 $09.01 .02 .02 /$ SR04 $09.01 .02 .02 / S R 11$ $09.01 .03 .01 /$ SR13 $09.01 .03 .02 /$ SR13 $09.01 .10 .01 /$ SR03 $09.01 .10 .02 /$ SR03 $11.02 /$ SR02

\#F 153 <initialize locator>:

09.01.01.01/SR03 09.01.01.02/SR03 09.01.02.01/SR0 4 $09.01 .02 .01 /$ SR11 $09.01 .02 .02 /$ SR0 $409.01 .02 .02 / S R 11$ $09.01 .03 .01 /$ SR13 $09.01 .03 .02 /$ SR13 $09.01 .10 .01 /$ SR0 3 $09.01 .10 .02 /$ SR03 $11.02 /$ SR02

\#F 154 <initialize stroke 3>: 09.01.01.01/SR03 09.01.01.02/SR03 09.01.02.01/SR0 4 09.01.02.01/SR11 09.01.02.02/SR04 09.01.02.02/SR11 $09.01 .03 .01 /$ SR13 $09.01 .03 .02 /$ SR13 $09.01 .10 .01 /$ SR0 3 $09.01 .10 .02 /$ SR03 $11.02 /$ SR02

\#F 155 <initialize stroke>:

09.01.01.01/SR03 09.01.01.02/SR03 09.01.02.01/SR0 4 $09.01 .02 .01 /$ SR11 $09.01 .02 .02 /$ SR0 4 09.01.02.02/SR11 $09.01 .03 .01 /$ SR13 $09.01 .03 .02 /$ SR13 $09.01 .10 .01 /$ SR03 $09.01 .10 .02 /$ SR03 $11.02 /$ SR02

\#F 156 <initialize valuator 3>:

09.01.01.01/SR03 09.01.01.02/SR03 09.01.02.01/SR0 4 $09.01 .02 .01 /$ SR11 $09.01 .02 .02 /$ SR0 $409.01 .02 .02 / S R 11$ $09.01 .10 .01 /$ SR03 09.01.10.02/SR03 11.02/SR02

\#F 157 <initialize valuator>: 
$09.01 .01 .01 /$ SR03 09.01.01.02/SR03 $09.01 .02 .01 /$ SR11 $09.01 .02 .02 /$ SR0 4 $09.01 .10 .01 /$ SR03 09.01.10.02/SR03

\#F 158 <initialize choice 3>:

$09.01 .01 .01 /$ SR03 09.01.01.02/SR03 $09.01 .02 .01 /$ SR11 $09.01 .02 .02 /$ SR0 4 $09.01 .10 .01 /$ SR01 $09.01 .10 .01 /$ SR03 $09.01 .10 .02 /$ SR0 3 09.01.11.01/SR0 4

\#F 159 <initialize choice>:

09.01.01.01/SR03 09.01.01.02/SR03 $09.01 .02 .01 /$ SR11 $09.01 .02 .02 /$ SR0 4 $09.01 .10 .01 /$ SR01 $09.01 .10 .01 /$ SR03 $09.01 .10 .02 /$ SR03 09.01.11.01/SR0 4

\#F 160 <initialize pick 3>:

09.01.01.01/SR03 09.01.01.02/SR03 $09.01 .02 .01 /$ SR10 $09.01 .02 .02 /$ SR0 4 $09.01 .10 .01 /$ SR01 $09.01 .10 .01 /$ SR03 $09.01 .10 .02 /$ SR03 09.01.11.01/SR0 4

\#F 161 <initialize pick>:

09.01.01.01/SR03 09.01.01.02/SR03 $09.01 .02 .01 /$ SR10 $09.01 .02 .02 /$ SR0 4 $09.01 .10 .01 /$ SR01 $09.01 .10 .01 /$ SR03 $09.01 .10 .02 /$ SR03 09.01.11.01/SR0 4

$09.01 .02 .01 / \mathrm{SR} 04$

$09.01 .02 .02 /$ SR10 $09.01 .10 .02 /$ SR01 $09.01 .11 .02 / \mathrm{SR} 0111.02 / \mathrm{SR} 02$

\#F 162 <initialize string 3>:

09.01.01.01/SR03 09.01.01.02/SR03 $09.01 .02 .01 /$ SR11 $09.01 .02 .02 /$ SR0 4 $09.01 .10 .01 /$ SR03 09.01.10.02/SR03

$09.01 .02 .01 / \mathrm{SR} 04$ $09.01 .02 .02 / \mathrm{SR} 11$ $11.02 /$ SR02

\#F 163 <initialize string>:

09.01.01.01/SR03 09.01.01.02/SR03 09.01.02.01/SR0 4 $09.01 .02 .01 /$ SR11 $09.01 .02 .02 /$ SR0 $409.01 .02 .02 / S R 11$ $09.01 .10 .01 /$ SR03 09.01.10.02/SR03 $11.02 /$ SR02

\#F 164 <set locator mode>:

05.02/SR30 09.01.01.01/SR03 09.01.01.02/SR03 09.01.02.01/SR04 $09.01 .02 .01 /$ SR11 $09.01 .02 .02 /$ SR0 4 09.01.02.02/SR11 $09.01 .10 .01 /$ SR01 09.01.10.02/SR01 09.01.11.01/SR0 4 $09.01 .11 .02 / \mathrm{SR} 0111.02 / \mathrm{SR} 02$

\#F 165 <set stroke mode>:

05.02/SR30 09.01.01.01/SR03 09.01.01.02/SR03 09.01.02.01/SR04 09.01.02.01/SR11 09.01.02.02/SR04 09.01.02.02/SR11 $09.01 .10 .01 /$ SR01 $09.01 .10 .02 /$ SR01 09.01.11.01/SR0 4 $09.01 .11 .02 /$ SR01 $11.02 /$ SR02

\#F 166 <set valuator mode>:

05.02/SR30 09.01.01.01/SR03 09.01.01.02/SR03 09.01.02.01/SR04 $09.01 .02 .01 /$ SR11 $09.01 .02 .02 /$ SR0 $409.01 .02 .02 / S R 11$ $09.01 .10 .01 / \mathrm{SR} 0109.01 .10 .02 / \mathrm{SR} 01$ 09.01.11.01/SR0 4 
$09.01 .11 .02 / \mathrm{SR} 0111.02 / \mathrm{SR} 02$

\#F 167 <set choice mode>:

05.02/SR30 09.01.01.01/SR03 09.01.01.02/SR03 09.01.02.01/SR0 4 $09.01 .02 .01 /$ SR11 $09.01 .02 .02 /$ SR0 $409.01 .02 .02 /$ SR 11

$09.01 .10 .01 / \mathrm{SR} 0109.01 .10 .02 / \mathrm{SR} 0109.01 .11 .01 / \mathrm{SR} 04$

$09.01 .11 .02 /$ SR01 $11.02 / S R 02$

\#F 168 <set pick mode>:

05.02/SR30 09.01.01.01/SR03 09.01.01.02/SR03 09.01.02.01/SR04

$09.01 .02 .01 /$ SR10 $09.01 .02 .02 /$ SR0 $409.01 .02 .02 /$ SR10

$09.01 .10 .01 /$ SR01 $09.01 .10 .02 /$ SR01 09.01.11.01/SR0 4

$09.01 .11 .02 /$ SR01 $11.02 /$ SR02

\#F 169 <set string mode>:

05.02/SR30 09.01.01.01/SR03 09.01.01.02/SR03 09.01.02.01/SR04 $09.01 .02 .01 /$ SR11 $09.01 .02 .02 /$ SR0 4 09.01.02.02/SR11

$09.01 .10 .01 /$ SR01 $09.01 .10 .02 /$ SR01 $09.01 .11 .01 / S R 04$

$09.01 .11 .02 /$ SR01 11.02/SR02

\#F 170 <request locator $3>$ :

05.02/SR30 09.01.01.01/SR03 09.01.01.02/SR03 09.01.02.01/SR04

09.01.02.01/SR11 09.01.02.02/SR04 09.01.02.02/SR11 $11.02 /$ SR02

\#F 171 <request locator>:

05.02/SR30 09.01.01.01/SR03 09.01.01.02/SR03 09.01.02.01/SR04

09.01.02.01/SR11 09.01.02.02/SR04 09.01.02.02/SR11 $11.02 /$ SR02

\#F 172 <request stroke $3>$ :

05.02/SR30 09.01.01.01/SR03 09.01.01.02/SR03 09.01.02.01/SR04 $09.01 .02 .01 /$ SR11 $09.01 .02 .02 /$ SR0 $4 \quad 09.01 .02 .02 / S R 1111.02 / S R 02$

\#F 173 <request stroke>:

05.02/SR30 09.01.01.01/SR03 09.01.01.02/SR03 09.01.02.01/SR04 $09.01 .02 .01 /$ SR $1009.01 .02 .02 /$ SR0 $4 \quad 09.01 .02 .02 /$ SR $1011.02 / S R 02$

\#F 174 <request valuator>:

05.02/SR30 09.01.01.01/SR03 09.01.01.02/SR03 09.01.02.01/SR04 $09.01 .02 .01 /$ SR11 $09.01 .02 .02 /$ SR0 $409.01 .02 .02 / S R 1111.02 / S R 02$

\#F 175 <request choice>:

05.02/SR30 09.01.01.01/SR03 09.01.01.02/SR03 09.01.02.01/SR04 $09.01 .02 .01 /$ SR11 $09.01 .02 .02 /$ SR0 $409.01 .02 .02 / S R 1111.02 / S R 02$

\#F 176 <request pick>:

$05.02 /$ SR30 09.01.01.01/SR03 09.01.01.02/SR03 09.01.02.01/SR04 $09.01 .02 .01 /$ SR10 $09.01 .02 .02 /$ SR0 $409.01 .02 .02 / S R 1011.02 / S R 02$

\#F 177 <request string>:

05.02/SR30 09.01.01.01/SR03 09.01.01.02/SR03 09.01.02.01/SR04 09.01.02.01/SR11 09.01.02.02/SR0 4 09.01.02.02/SR11 $11.02 /$ SR02

\# 178 <sample locator 3>:

$09.01 .01 .01 /$ SR03 09.01.01.02/SR03 09.01.02.01/SR0 4 
09.01.02.01/SR11 09.01.02.02/SR04 09.01.02.02/SR11 $11.02 / S R 02$

\#F 179 <sample locator>:

09.01.01.01/SR03 09.01.01.02/SR03 09.01.02.01/SR0 4

09.01.02.01/SR11 09.01.02.02/SR04 09.01.02.02/SR11 $11.02 / S R 02$

\#F 180 <sample stroke $3>$ :

09.01.01.01/SR03 09.01.01.02/SR03 09.01.02.01/SR0 4

09.01.02.01/SR11 09.01.02.02/SR04 09.01.02.02/SR11 11.02/SR02

\#F 181 <sample stroke>:

$09.01 .01 .01 /$ SR03 $09.01 .01 .02 /$ SR0 $309.01 .02 .01 /$ SR0 4

09.01.02.01/SR11 09.01.02.02/SR0 4 09.01.02.02/SR11 11.02/SR02.

\#F 182 <sample valuator>:

09.01.01.01/SR03 09.01.01.02/SR03 09.01.02.01/SR0 4

09.01.02.01/SR11 09.01.02.02/SR0 4 09.01.02.02/SR11 $11.02 /$ SR02

\#F 183 <sample choice>:

09.01.01.01/SR03 09.01.01.02/SR03 09.01.02.01/SR0 4

09.01.02.01/SR11 09.01.02.02/SR0 4 09.01.02.02/SR11 $11.02 / S R 02$

\#F 184 <sample pick>:

09.01.01.01/SR03 09.01.01.02/SR03 09.01.02.01/SR0 4

09.01.02.01/SR10 09.01.02.02/SR0 $409.01 .02 .02 /$ SR10

$11.02 /$ SR02

\#F 185 <sample string>:

09.01.01.01/SR03 09.01.01.02/SR03 09.01.02.01/SR0 4

09.01.02.01/SR11 09.01.02.02/SR04 09.01.02.02/SR11 $11.02 / S R 02$

\#F 186 <await event>:

09.01.01.01/SR03 09.01.01.02/SR03 11.02/SR02

\#F 187 <flush device events>:

09.01.01.01/SR03 09.01.01.02/SR03 09.01.02.01/SR0 4

09.01.02.01/SR11 09.01.02.02/SR04 09.01.02.02/SR11

09.01.10.01/SR01 09.01.10.02/SR01 09.01.11.01/SR0 4

$09.01 .11 .02 /$ SR01 $11.02 /$ SR02

\#F 188 <get locator 3>:

09.01.01.01/SR03 09.01.01.02/SR03 11.02/SR02

\#F 189 <get locator $>$ :

09.01.01.01/SR03 09.01.01.02/SR03 11.02/SR02

\#F 190 <get stroke 3>:

09.01.01.01/SR03 09.01.01.02/SR03 09.01.10.01/SR02

$09.01 .10 .02 /$ SR02 $11.02 /$ SR02

\#F 191 <get stroke>:

09.01.01.01/SR03 09.01.01.02/SR03 09.01.10.01/SR02

$09.01 .10 .02 /$ SR02 $11.02 /$ SR02

\#F 192 <get valuator>: 
09.01.01.01/SR03 09.01.01.02/SR03 11.02/SR02

\#F 193 <get choice>:

09.01.01.01/SR03 09.01.01.02/SR03 11.02/SR02

\#F 194 <get pick>:

09.01.01.01/SR03 09.01.01.02/SR03 09.01.10.01/SR02

$09.01 .10 .02 /$ SR02 $11.02 /$ SR02

\#F 195 <get string>:

09.01.01.01/SR03 09.01.01.02/SR03 11.02/SR02

\#F 196 <write item to metafile>:

09.01.01.01/SR03 09.01.01.02/SR03 09.01.02.01/SR0 4

$09.01 .02 .01 /$ SR0 $609.01 .02 .02 /$ SR0 $409.01 .02 .02 /$ SR0 6

09.01.10.01/SR03 09.01.10.02/SR03 11.02/SR02

\#F 197 <get item type from metafile>:

09.01.01.01/SR03 09.01.01.02/SR03 09.01.02.01/SR04

$09.01 .02 .01 /$ SR08 $09.01 .02 .02 /$ SR0 $409.01 .02 .02 /$ SR0 $811.02 / S R 02$

\#F 198 <read item from metafile>:

09.01.01.01/SR03 09.01.01.02/SR03 09.01.02.01/SR0 4

09.01.02.01/SR08 09.01.02.02/SR04 09.01.02.02/SR08 11.02/SR02

\#F 199 <interpret item>:

09.01.01.01/SR02 09.01.01.02/SR02 09.01.10.01/SR03

$09.01 .10 .02 /$ SR03 $11.02 /$ SR02

\#F 200 <inquire system state value>:

$01 /$ SR0 $311.02 /$ SR0 2

\#F 201 <inquire workstation state value>:

05.01.01/SR02 05.01.01/SR03 05.01.01/SR04 05.01.01/SR05

11. $02 / \mathrm{SR} 02$

\#F 202 <inquire structure state value>:

$02.02 .01 /$ SR09 $11.02 /$ SR02

\#E 203 <inquire archive state value>:

03.01/SR03 03.01/SR05 03.01/SR08 03.01/SR10 11.02/SR02

\#E 204 <inquire list of available workstation types>:

05.01.02/SR04 05.01.02/SR05 05.01.02/SR06 05.01.02/SR07

$09.02 .01 /$ SR01 09.02.10/SR03 09.02.11/SR01 09.02.11/SR02

11. $02 / \mathrm{SR} 02$

\#F 205 <inquire phigs facilities>:

$02.03 .03 /$ SR21 02.03.03/SR22 03.01/SR01 03.01/SR02 $04.01 .03 /$ SR0 7

$04.01 .03 /$ SR0 $804.03 .04 .01 /$ SR0 $0404.03 .04 .01 /$ SR08 $05.01 .01 /$ SR0 9

$05.01 .01 /$ SR10 05.01.01/SR11 09.02.01/SR01 $09.02 .10 /$ SR03

$09.02 .11 /$ SR01 $09.02 .11 /$ SR02 $11.02 /$ SR02

\#F 206 <inquire generalized structure element facilities>: 
$02.02 .02 / S R 1102.02 .02 / S R 1209.02 .01 / S R 0109.02 .10 /$ SR0 3

$09.02 .11 /$ SR01 $09.02 .11 /$ SR02 $11.02 /$ SR02

\#F 207 <inquire modelling clipping facilities>:

$06.01 .02 /$ SR35 $06.01 .02 /$ SR36 $06.01 .02 /$ SR37 $09.02 .01 /$ SR01

$09.02 .10 /$ SR03 09.02.11/SR01 09.02.11/SR02 $11.02 /$ SR02

\#F 208 <inquire edit mode>:

$02.02 .03 /$ SR11 09.02.01/SR01 $11.02 /$ SR02

\#F 209 <inquire set of open workstations>:

05.01.01/SR01 05.01.01/SR06 05.01.01/SR07 05.01.01/SR12

$09.02 .01 /$ SR01 09.02.10/SR03 09.02.11/SR01 09.02.11/SR02 $11.02 / \mathrm{SR} 02$

\#F 210 <inquire structure identifiers>:

02.01/SR02 02.01.01/SR09 09.02.01/SR01 09.02.10/SR03

$09.02 .11 /$ SR01 09.02.11/SR02 11.02/SR02

\#F 211 <inquire archive files>:

03.01/SR0 4 03.01/SR06 03.01/SR09 03.01/SR11 09.02.01/SR01

$09.02 .10 /$ SR03 $11.02 /$ SR02

\#F 212 <inquire conflict resolution>:

$03.02 /$ SR01 03.02/SR03 09.02.01/SR01 11.02/SR02

\#F 213 <inquire all conflicting structures>:

03.04/SR02 09.02.01/SR04 09.02.09/SR01 09.02.10/SR03

$09.02 .11 /$ SR01 09.02.11/SR02 11.02/SR02

\#F 214 <inquire conflicting structures in network>:

03.04/SR03 03.04/SR04 09.02.01/SR0 $409.02 .05 /$ SR01 09.02.09/SR01

$09.02 .10 /$ SR01 09.02.10/SR03 09.02.11/SR01 09.02.11/SR02

$09.02 .11 / \mathrm{SR} 0311.02 / \mathrm{SR} 02$

\#F 215 <inquire more simultaneous events>:

$09.02 .01 /$ SR01 $11.02 /$ SR02

\#F 216 <inquire workstation connection and type>:

$05.01 .02 /$ SR0 8 09.02.01/SR02 09.02.02/SR03 11.02/SR02

\#F 217 <inquire list of view indices>:

$06.02 .02 /$ SR08 $06.02 .02 /$ SR18 $06.02 .02 /$ SR19 $06.02 .02 /$ SR20

$09.02 .01 /$ SR02 $09.02 .02 /$ SR03 $09.02 .02 /$ SR0 $409.02 .10 /$ SR0 3

$09.02 .11 /$ SR01 09.02.11/SR02 11.02/SR02

\#F 218 <inquire view representation>:

$06.02 .02 /$ SR09 $06.02 .02 /$ SR10 $06.02 .02 /$ SR11 $06.02 .02 /$ SR 17

$06.02 .02 / S R 2406.02 .02 / S R 25 \quad 06.02 .02 / S R 26 \quad 06.02 .02 /$ SR2 7

$06.02 .02 / S R 2806.02 .02 / S R 2909.02 .01 /$ SR02 $09.02 .02 /$ SR0 3

$09.02 .02 /$ SR0 $409.02 .03 /$ SR0 $209.02 .03 /$ SR0 $809.02 .10 /$ SR0 1

$09.02 .11 /$ SR03 $11.02 /$ SR02

\#F 219 <inquire hlhsr mode>: 
$04.03 .03 /$ SR0 $504.03 .03 /$ SR0 $604.03 .03 /$ SR0 $704.03 .03 /$ SR11 $04.03 .03 /$ SR12 $04.03 .03 /$ SR13 $04.03 .03 /$ SR1 $4 \quad 04.03 .03 /$ SR15 $09.02 .01 /$ SR02 $09.02 .02 /$ SR03 $09.02 .02 /$ SR0 $411.02 /$ SR0 2

\#F 220 <inquire posted structures>:

$05.03 /$ SR14 05.03/SR15 09.02.01/SR02 09.02.02/SR03 09.02.02/SR05 $09.02 .10 /$ SR03 09.02.11/SR01 09.02.11/SR02 $11.02 /$ SR0 2

\#F 221 <inquire display update state>:

$04.03 .03 /$ SR0 $604.03 .03 /$ SR0 $705.02 /$ SR01 $05.02 /$ SR0 $205.02 / S R 04$ 05.02/SR11 $05.02 / S R 1205.02 / S R 1305.02 / S R 1405.02 / S R 1505.02 / S R 22$ $05.02 /$ SR2 $305.02 / S R 2405.02 / S R 2505.02 / S R 2605.02 / S R 2705.02 / S R 28$ $05.02 / S R 2905.02 / S R 3105.02 / S R 32 \quad 06.02 .02 / S R 2706.02 .02 / S R 28$ $06.03 /$ SR18 06.03/SR19 $09.02 .01 /$ SR02 $09.02 .02 /$ SR03 $09.02 .02 /$ SR0 5 $11.02 / \mathrm{SR} 02$

\#F 222 <inquire list of polyline indices>: $04.02 .01 .02 /$ SR12 $04.02 .01 .02 /$ SR13 $04.02 .01 .02 /$ SR1 6 $04.02 .01 .02 /$ SR2 $404.02 .01 .02 /$ SR25 $09.02 .01 /$ SR02 $09.02 .02 /$ SR03 $09.02 .02 /$ SR05 09.02.10/SR03 09.02.11/SR01 09.02.11/SR02 $11.02 / \mathrm{SR} 02$

\#F 223 <inquire polyline representation>: $04.02 .01 .02 /$ SR13 $04.02 .01 .02 /$ SR14 $04.02 .01 .02 /$ SR15 $04.02 .01 .02 /$ SR16 $04.02 .01 .02 /$ SR2 $404.02 .01 .02 /$ SR2 5 $04.02 .01 .02 / S R 48 \quad 04.02 .01 .02 /$ SR4 $909.02 .01 /$ SR02 $09.02 .02 / S R 03$ $09.02 .02 /$ SR05 09.02.03/SR01 09.02.03/SR02 09.02.10/SR01 $09.02 .11 / \mathrm{SR} 0311.02 / \mathrm{SR} 02$

\#F 224 <inquire list of polymarker indices>: $04.02 .02 .02 /$ SR12 $04.02 .02 .02 /$ SR13 $04.02 .02 .02 /$ SR1 6 $04.02 .02 .02 /$ SR2 $4 \quad 04.02 .02 .02 /$ SR25 $09.02 .01 /$ SR02 $09.02 .02 /$ SR03 $09.02 .02 /$ SR0 $509.02 .10 /$ SR03 09.02.11/SR01 09.02.11/SR02 $11.02 / \mathrm{SR} 02$

\# 225 <inquire polymarker representation>:

$04.02 .02 .02 /$ SR13 $04.02 .02 .02 /$ SR14 $04.02 .02 .02 /$ SR15

$04.02 .02 .02 /$ SR1 $604.02 .02 .02 /$ SR2 $4 \quad 04.02 .02 .02 /$ SR2 5

$04.02 .02 .02 /$ SR4 $904.02 .02 .02 /$ SR50 $09.02 .01 /$ SR02 $09.02 .02 /$ SR0 3 $09.02 .02 /$ SR05 09.02.03/SR01 09.02.03/SR02 09.02.10/SR01 $09.02 .11 /$ SR03 $11.02 /$ SR02

\#F 226 <inquire list of text indices>: $04.02 .03 .02 /$ SR12 $04.02 .03 .02 /$ SR13 $04.02 .03 .02 /$ SR1 6 $04.02 .03 .02 / S R 24 \quad 04.02 .03 .02 / S R 25 \quad 04.02 .04 .02 / S R 09 \quad 09.02 .01 / S R 02$ $09.02 .02 /$ SR03 09.02.02/SR05 09.02.10/SR03 09.02.11/SR01 $09.02 .11 / \mathrm{SR} 0211.02 / \mathrm{SR} 02$

\#E 227 <inquire text representation>:

$04.02 .03 .02 /$ SR13 $04.02 .03 .02 /$ SR14 $04.02 .03 .02 /$ SR15

$04.02 .03 .02 /$ SR1 $604.02 .03 .02 /$ SR2 $4 \quad 04.02 .03 .02 /$ SR2 5

$04.02 .03 .02 /$ SR2 $804.02 .03 .02 /$ SR $34 \quad 04.02 .03 .02 /$ SR 35

$04.02 .03 .02 /$ SR $4704.02 .03 .02 /$ SR5 $504.02 .03 .02 /$ SR 65

$04.02 .03 .02 /$ SR $6604.02 .04 .02 /$ SR0 $904.02 .04 .02 /$ SR 10 
$04.02 .04 .02 / \mathrm{SR} 20 \quad 04.02 .04 .02 / \mathrm{SR} 30 \quad 04.02 .04 .02 / \mathrm{SR} 3109.02 .01 / \mathrm{SR} 02$ $09.02 .02 /$ SR03 09.02.02/SR05 09.02.03/SR01 09.02.03/SR02

$09.02 .10 /$ SR01 $09.02 .11 /$ SR03 $11.02 /$ SR02

\#F 228 <inquire list of interior indices>:

$04.02 .05 .02 /$ SR13 $04.02 .05 .02 /$ SR14 $04.02 .05 .02 /$ SR1 7

$04.02 .05 .02 / S R 2504.02 .05 .02 / S R 26 \quad 09.02 .01 /$ SR02 $09.02 .02 / S R 03$

$09.02 .02 /$ SR05 09.02.10/SR03 09.02.11/SR01 09.02.11/SR02

$11.02 / \mathrm{SR} 02$

\#F 229 <inquire interior representation>:

$04.02 .05 .02 /$ SR14 $04.02 .05 .02 /$ SR15 $04.02 .05 .02 /$ SR1 6

$04.02 .05 .02 /$ SR17 $04.02 .05 .02 /$ SR25 $04.02 .05 .02 /$ SR2 6

$04.02 .05 .02 /$ SR31 $04.02 .05 .02 /$ SR $40 \quad 04.02 .05 .02 /$ SR 41

$04.02 .05 .02 /$ SR52 $04.02 .05 .02 /$ SR53 $09.02 .01 /$ SR02 $09.02 .02 /$ SR03

$09.02 .02 /$ SR05 09.02.03/SR01 09.02.03/SR02 09.02.10/SR01

$09.02 .11 /$ SR03 $11.02 /$ SR02

\#F 230 <inquire list of edge indices>:

$04.02 .05 .03 /$ SR12 $04.02 .05 .03 /$ SR13 $04.02 .05 .03 /$ SR 16

$04.02 .05 .03 /$ SR24 $04.02 .05 .03 /$ SR25 $09.02 .01 /$ SR02 $09.02 .02 / S R 03$

$09.02 .02 /$ SR05 $09.02 .10 /$ SR03 09.02.11/SR01 $09.02 .11 /$ SR02

$11.02 /$ SR02

\#F 231 <inquire edge representation>:

$04.02 .05 .03 /$ SR13 $04.02 .05 .03 /$ SR14 $04.02 .05 .03 /$ SR15

$04.02 .05 .03 /$ SR1 $604.02 .05 .03 /$ SR2 $404.02 .05 .03 /$ SR2 5

$04.02 .05 .03 /$ SR29 $04.02 .05 .03 /$ SR51 $04.02 .05 .03 /$ SR52 $09.02 .01 /$ SR02

$09.02 .02 /$ SR03 $09.02 .02 /$ SR0 $509.02 .03 /$ SR0 $109.02 .03 /$ SR0 2

$09.02 .10 /$ SR01 09.02.11/SR03 $11.02 /$ SR02

\#F 232 <inquire list of pattern indices>:

$04.02 .05 .01 /$ SR20 $04.02 .05 .01 /$ SR21 $04.02 .05 .04 /$ SR0 3

$04.02 .05 .04 /$ SR0 $404.02 .05 .04 /$ SR0 $704.02 .05 .04 /$ SR 16

$04.02 .05 .04 /$ SR $1709.02 .01 /$ SR02 $09.02 .02 /$ SR0 $309.02 .02 / S R 05$

$09.02 .10 /$ SR03 09.02.11/SR01 09.02.11/SR02 $11.02 /$ SR02

\#F 233 <inquire pattern representation>:

$04.02 .05 .01 /$ SR20 $04.02 .05 .04 /$ SR0 $404.02 .05 .04 /$ SR0 5

$04.02 .05 .04 /$ SR0 $604.02 .05 .04 /$ SR0 $04.02 .05 .04 /$ SR 16

$04.02 .05 .04 /$ SR1 $704.02 .05 .04 /$ SR20 $04.02 .05 .04 /$ SR2 $109.02 .01 /$ SR02

$09.02 .02 /$ SR03 $09.02 .02 /$ SR05 $09.02 .03 /$ SR02 $09.02 .03 /$ SR0 5

$09.02 .03 /$ SR0 $69.02 .10 /$ SR01 09.02.10/SR02 $09.02 .11 /$ SR03

$11.02 / S R 02$

\#F 234 <inquire colour model>:

$04.03 .02 .01 /$ SR10 $04.03 .02 .02 /$ SR17 $04.03 .02 .02 /$ SR20 $09.02 .01 /$ SR02 $09.02 .02 /$ SR03 $09.02 .02 /$ SR05 $11.02 /$ SR0 2

\#F 235 <inquire list of colour indices>:

$04.02 .01 .02 /$ SR $4804.02 .01 .02 /$ SR49 $04.02 .02 .02 /$ SR 49

$04.02 .02 .02 /$ SR50 $04.02 .03 .02 /$ SR $6504.02 .03 .02 /$ SR 66

$04.02 .04 .02 /$ SR30 $04.02 .04 .02 /$ SR31 $04.02 .05 .02 /$ SR5 2

$04.02 .05 .02 /$ SR53 $04.02 .05 .03 /$ SR51 $04.02 .05 .03 /$ SR5 2 
$04.03 .02 .01 /$ SR03 $04.03 .02 .01 /$ SR04 $04.03 .02 .01 /$ SR0 6 $04.03 .02 .02 /$ SR13 $04.03 .02 .02 /$ SR14 $09.02 .01 /$ SR02 $09.02 .02 /$ SR0 3 $09.02 .02 /$ SR0 $509.02 .10 /$ SR03 09.02.11/SR01 09.02.11/SR02 11. $02 / \mathrm{SR} 02$

\#E 236 <inquire colour representation>:

$04.03 .02 .01 /$ SR0 $404.03 .02 .01 /$ SR0 $504.03 .02 .01 /$ SR0 6

$04.03 .02 .02 /$ SR0 $304.03 .02 .02 /$ SR0 $504.03 .02 .02 /$ SR0 6

$04.03 .02 .02 /$ SR13 $04.03 .02 .02 /$ SR14 $04.03 .02 .02 /$ SR2 1

$04.03 .02 .02 /$ SR2 $604.03 .02 .02 /$ SR30 $04.03 .02 .02 /$ SR 31

$04.03 .02 .02 /$ SR32 $04.03 .02 .02 /$ SR33 $09.02 .01 /$ SR02 $09.02 .02 /$ SR03

$09.02 .02 / \mathrm{SR} 0509.02 .03 / \mathrm{SR} 02 \quad 09.02 .03 / \mathrm{SR} 0709.02 .10 / \mathrm{SR} 01$

$09.02 .10 /$ SR02 09.02.11/SR03 $11.02 /$ SR02

\#F 237 <inquire highlighting filter>:

$04.03 .04 .02 /$ SR02 $04.03 .04 .02 /$ SR03 $09.02 .01 /$ SR02 $09.02 .02 / S R 03$

$09.02 .02 /$ SR05 $09.02 .10 /$ SR02 $11.02 /$ SR02

\#F 238 <inquire invisibility filter>:

$04.03 .04 .02 /$ SR0 $604.03 .04 .02 /$ SR07 09.02.01/SR02 09.02.02/SR03 $09.02 .02 /$ SR0 $509.02 .10 /$ SR02 $11.02 /$ SR0 2

\#F 239 <inquire workstation transformation 3>:

$06.03 /$ SR12 06.03/SR15 $06.03 /$ SR16 $06.03 /$ SR17 $06.03 /$ SR18 $06.03 /$ SR19

$06.03 /$ SR20 09.02.01/SR02 09.02.02/SR03 09.02.02/SR0 $411.02 / S R 02$

\#F 240 <inquire workstation transformation>:

$06.03 /$ SR13 06.03/SR15 $06.03 /$ SR16 $06.03 /$ SR17 $06.03 /$ SR1 $806.03 /$ SR1 9

$06.03 /$ SR20 09.02.01/SR02 09.02.02/SR03 09.02.02/SR0 $411.02 /$ SR02

\#F 241 <inquire locator device state 3>:

$09.02 .01 /$ SR02 09.02.02/SR03 09.02.02/SR07 09.02.10/SR01

$09.02 .10 /$ SR02 $09.02 .11 /$ SR03 $11.02 /$ SR02

\#F 242 <inquire locator device state>:

09.02.01/SR02 09.02.02/SR03 09.02.02/SR07 09.02.10/SR01

$09.02 .10 /$ SR02 09.02.11/SR03 $11.02 /$ SR02

\#F 243 <inquire stroke device state $3>$ :

09.02.01/SR02 09.02.02/SR03 09.02.02/SR07 09.02.10/SR02

$09.02 .11 /$ SR03 $11.02 / \mathrm{SR} 02$

\#F 244 <inquire stroke device state>:

09.02.01/SR02 09.02.02/SR03 09.02.02/SR07 09.02.10/SR02

$09.02 .11 /$ SR03 $11.02 /$ SR02

\#F 245 <inquire valuator device state 3>:

09.02.01/SR02 09.02.02/SR03 09.02.02/SR07 09.02.10/SR02 $11.02 /$ SR02

\#F 246 <inquire valuator device state>:

09.02.01/SR02 09.02.02/SR03 09.02.02/SR07 09.02.10/SR02

11. $02 / \mathrm{SR} 02$ 
\#F 247 <inquire choice device state 3>:

$09.02 .01 /$ SR02 09.02.02/SR03 09.02.02/SR07 09.02.10/SR02 $11.02 / \mathrm{SRO} 2$

\#F 248 <inquire choice device state>:

09.02.01/SR02 09.02.02/SR03 09.02.02/SR07 09.02.10/SR02 $11.02 / S R 02$

\#F 249 <inquire pick device state $3>$ :

09.02.01/SR02 09.02.02/SR03 09.02.02/SR06 09.02.10/SR01 $09.02 .10 /$ SR02 09.02.11/SR03 $11.02 /$ SR02

\#F 250 <inquire pick device state>:

09.02.01/SR02 09.02.02/SR03 09.02.02/SR06 09.02.10/SR01 $09.02 .10 /$ SR02 09.02.11/SR03 $11.02 /$ SR02

\#F 251 <inquire string device state 3>:

09.02.01/SR02 09.02.02/SR03 09.02.02/SR07 09.02.10/SR01 $09.02 .10 / \mathrm{SR} 0211.02 / \mathrm{SR} 02$

\#F 252 <inquire string device state>:

09.02.01/SR02 09.02.02/SR03 09.02.02/SR07 09.02.10/SR01 $09.02 .10 / \mathrm{SR} 0211.02 / \mathrm{SR} 02$

\#F 253 <inquire workstation category>:

$05.01 .02 /$ SR01 $05.01 .02 /$ SR02 $05.01 .02 /$ SR03 $05.01 .02 /$ SR0 4 05.01.02/SR10 09.02.01/SR01 09.02.02/SR01 09.02.02/SR02 $11.02 / S R 02$

\#F 254 <inquire display space size 3>:

05.01.02/SR01 06.03/SR02 06.03/SR04 09.02.01/SR01 09.02.02/SR01 $09.02 .02 /$ SR02 09.02.02/SR04 09.02.02/SR08 $11.02 /$ SR02

\#F 255 <inquire display space size>:

$04.01 /$ SR02 05.01.02/SR01 06.03/SR03 06.03/SR04 09.02.01/SR01 $09.02 .02 / S R 0109.02 .02 / S R 02 \quad 09.02 .02 / S R 04 \quad 09.02 .02 / S R 08$ $11.02 / \mathrm{SR} 02$

\#F 256 <inquire hlhsr facilities>:

$04.03 .03 /$ SR0 $84.03 .03 /$ SR09 $04.03 .03 /$ SR10 $04.03 .03 /$ SR11

$05.01 .02 /$ SR01 $09.02 .01 /$ SR01 $09.02 .02 /$ SR01 $09.02 .02 /$ SR02

$09.02 .02 /$ SR0 $409.02 .02 /$ SR0 $809.02 .10 /$ SR03 $09.02 .11 /$ SR01

$09.02 .11 / \mathrm{SR} 0211.02 / \mathrm{SR} 02$

\#F 257 <inquire view facilities>:

05.01.02/SR01 06.02.02/SR04 06.02.02/SR05 09.02.01/SR01

$09.02 .02 / S R 01 \quad 09.02 .02 / S R 02 \quad 09.02 .02 / S R 04 \quad 09.02 .02 / S R 08$

$11.02 /$ SR02

\#F 258 <inquire predefined view representation>:

$05.01 .02 /$ SR01 $06.02 .02 /$ SR0 $606.02 .02 /$ SR0 $0606.02 .02 /$ SR0 9

$09.02 .01 /$ SR01 $09.02 .02 /$ SR01 $09.02 .02 /$ SR02 $09.02 .02 /$ SR0 4

$09.02 .02 /$ SR0 $89.02 .03 /$ SR02 $09.02 .03 /$ SR0 $811.02 /$ SR0 2 
\#E 259 <inquire workstation classification>:

05.01.02/SR01 05.01.02/SR09 05.01.02/SR10 09.02.01/SR01

$09.02 .02 /$ SR01 $09.02 .02 /$ SR02 $09.02 .02 /$ SR0 $509.02 .02 /$ SR0 8 $11.02 / \mathrm{SR} 02$

\#F 260 <inquire dynamics of workstation attributes>: $04.03 .03 /$ SR0 $604.03 .03 /$ SR0 7 05.01.02/SR01 05.02/SR06 05.02/SR1 9 $05.02 / S R 2005.02 / S R 2105.02 / S R 2405.02 / S R 2505.02 / S R 2605.02 / S R 27$ $05.02 / S R 2805.02 / S R 2905.02 / S R 3105.02 / S R 3209.02 .01 / S R 01$ $09.02 .02 /$ SR01 09.02.02/SR02 09.02.02/SR05 09.02.02/SR0 8 $11.02 / \mathrm{SR} 02$

\#F 261 <inquire default display update state>: 05.01.02/SR01 05.02/SR01 05.02/SR02 05.02/SR05 09.02.01/SR01 09.02.02/SR01 09.02.02/SR02 09.02.02/SR05 09.02.02/SR0 8 $11.02 / \mathrm{SR} 02$

\#F 262 <inquire polyline facilities>:

$04.02 .01 .01 /$ SR0 $64.02 .01 .01 /$ SR0 $04.02 .01 .01 /$ SR15 $04.02 .01 .01 /$ SR1 $604.02 .01 .02 /$ SR19 $04.02 .01 .02 /$ SR2 1 $04.02 .01 .02 /$ SR2 $504.02 .01 .02 /$ SR2 $604.02 .01 .02 /$ SR2 7 $04.02 .01 .02 /$ SR2 $904.02 .01 .02 /$ SR30 $04.02 .01 .02 /$ SR3 1 $04.02 .01 .02 /$ SR32 $04.02 .01 .02 /$ SR33 $04.02 .01 .02 /$ SR 34 $04.02 .01 .02 /$ SR39 $04.02 .01 .02 /$ SR40 $04.02 .01 .02 /$ SR4 $1 \quad 05.01 .02 /$ SR01 $09.02 .01 /$ SR01 09.02.02/SR01 09.02.02/SR02 $09.02 .02 /$ SR0 5 09.02.02/SR08 09.02.10/SR03 09.02.11/SR01 09.02.11/SR02 $11.02 / \mathrm{SR} 02$

\#F 263 <inquire predefined polyline representation>: $04.02 .01 .02 /$ SR20 $04.02 .01 .02 /$ SR21 $04.02 .01 .02 /$ SR22 $04.02 .01 .02 / S R 2304.02 .01 .02 / S R 24 \quad 04.02 .01 .02 / S R 26$ $04.02 .01 .02 /$ SR27 $04.02 .01 .02 / S R 28 \quad 05.01 .02 / S R 01 \quad 09.02 .01 / S R 01$ $09.02 .02 /$ SR01 09.02.02/SR02 09.02.02/SR05 09.02.02/SR0 8 $09.02 .03 /$ SR01 $09.02 .03 /$ SR03 $11.02 /$ SR02

\#F 264 <inquire polymarker facilities>: $04.02 .02 .01 /$ SR0 $604.02 .02 .01 /$ SR0 $704.02 .02 .01 /$ SR 15 $04.02 .02 .01 /$ SR1 $604.02 .02 .02 /$ SR19 $04.02 .02 .02 /$ SR2 1 $04.02 .02 .02 /$ SR2 $504.02 .02 .02 /$ SR2 $604.02 .02 .02 /$ SR2 7 $04.02 .02 .02 /$ SR2 $904.02 .02 .02 /$ SR30 $04.02 .02 .02 /$ SR 31 $04.02 .02 .02 /$ SR32 $04.02 .02 .02 /$ SR33 $04.02 .02 .02 /$ SR 34 $04.02 .02 .02 /$ SR39 $04.02 .02 .02 /$ SR40 $04.02 .02 .02 /$ SR4 $1 \quad 05.01 .02 /$ SR01 $09.02 .01 /$ SR01 $09.02 .02 /$ SR01 $09.02 .02 /$ SR0 $209.02 .02 /$ SR0 5 $09.02 .02 /$ SR0 $09.02 .10 /$ SR03 09.02.11/SR01 09.02.11/SR02 $11.02 / \mathrm{SR} 02$

\#F 265 <inquire predefined polymarker representation>: $04.02 .02 .02 /$ SR20 $04.02 .02 .02 /$ SR21 $04.02 .02 .02 /$ SR2 2 $04.02 .02 .02 /$ SR23 $04.02 .02 .02 /$ SR2 $4 \quad 04.02 .02 .02 /$ SR2 6 $04.02 .02 .02 /$ SR27 $04.02 .02 .02 /$ SR2 8 05.01.02/SR01 $09.02 .01 /$ SR01 $09.02 .02 /$ SR01 09.02.02/SR02 09.02.02/SR05 09.02.02/SR0 8 $09.02 .03 /$ SR01 $09.02 .03 /$ SR03 $11.02 /$ SR0 2

\#F 266 <inquire text facilities>: 
$04.02 .03 .01 / \mathrm{SR} 21$ $04.02 .03 .01 /$ SR 37 $04.02 .03 .01 /$ SR55 $04.02 .03 .01 / \mathrm{SR} 61$ $04.02 .03 .02 / \mathrm{SR} 25$ $04.02 .03 .02 /$ SR30 $04.02 .03 .02 /$ SR33 $04.02 .03 .02 / \mathrm{SR} 36$ $04.02 .03 .02 / \mathrm{SR} 47$ $04.02 .03 .02 /$ SR53 $04.02 .03 .02 /$ SR 59 $04.02 .04 .01 / \mathrm{SR} 20$ $04.02 .04 .02 /$ SR10 $04.02 .04 .02 / \mathrm{SR} 26$ $09.02 .11 /$ SR01 09.02.11/SR02 $11.02 /$ SR02

$04.02 .03 .01 / \mathrm{SR} 2204.02 .03 .01 / \mathrm{SR} 28$ $04.02 .03 .01 /$ SR53 $04.02 .03 .01 /$ SR5 4 $04.02 .03 .01 /$ SR5 $604.02 .03 .01 /$ SR5 7 $04.02 .03 .02 /$ SR19 $04.02 .03 .02 / S R 21$ $04.02 .03 .02 /$ SR2 $604.02 .03 .02 / S R 27$ $04.02 .03 .02 /$ SR31 $04.02 .03 .02 /$ SR32 $04.02 .03 .02 /$ SR3 $404.02 .03 .02 /$ SR3 5 $04.02 .03 .02 /$ SR3 $304.02 .03 .02 / S R 40$ $04.02 .03 .02 /$ SR5 $104.02 .03 .02 /$ SR5 2 $04.02 .03 .02 /$ SR5 $404.02 .03 .02 /$ SR5 5 $04.02 .04 .01 /$ SR14 $04.02 .04 .01 /$ SR15 $04.02 .04 .01 /$ SR2 $904.02 .04 .01 /$ SR52 $04.02 .04 .02 /$ SR13 $04.02 .04 .02 / S R 20$ $09.02 .02 /$ SR02 $09.02 .02 /$ SR0 $509.02 .02 /$ SR0 $809.02 .10 /$ SR0 3 $05.01 .02 / S R 0109.02 .01 / S R 0109.02 .02 / S R 01$

\#F 267 <inquire predefined text representation>:

$04.02 .03 .02 /$ SR2 $004.02 .03 .02 /$ SR21 $04.02 .03 .02 /$ SR2 2 $04.02 .03 .02 /$ SR2 $304.02 .03 .02 /$ SR2 $404.02 .03 .02 / S R 26$ $04.02 .03 .02 /$ SR2 $704.02 .03 .02 /$ SR2 $804.02 .03 .02 /$ SR2 9 $04.02 .03 .02 /$ SR3 $404.02 .04 .02 /$ SR0 $804.02 .04 .02 /$ SR0 9 $04.02 .04 .02 /$ SR10 $05.01 .02 /$ SR01 $09.02 .01 /$ SR01 $09.02 .02 / S R 01$ $09.02 .02 /$ SR02 $09.02 .02 /$ SR0 $509.02 .02 /$ SR0 $809.02 .03 /$ SR01 $09.02 .03 /$ SR03 $11.02 /$ SR02

\#F 268 <inquire annotation facilities>: $04.02 .04 .01 /$ SR43 $04.02 .04 .01 /$ SR44 $04.02 .04 .01 / S R 45$ $04.02 .04 .01 / S R 4604.02 .04 .01 / S R 4704.02 .04 .01 / S R 77$ $04.02 .04 .01 / S R 7804.02 .04 .01 /$ SR79 $04.02 .04 .01 /$ SR 80 $04.02 .04 .01 /$ SR81 $04.02 .04 .01 /$ SR82 $05.01 .02 /$ SR01 $09.02 .01 / S R 01$ 09.02.02/SR01 09.02.02/SR02 09.02.02/SR05 09.02.02/SR0 8 $09.02 .10 /$ SR03 09.02.11/SR01 09.02.11/SR02 $11.02 /$ SR02

\#F 269 <inquire text extent>:

$04.02 .03 .01 /$ SR36 $04.02 .03 .01 /$ SR4 $204.02 .03 .01 /$ SR5 9 $04.02 .03 .02 /$ SR5 $804.02 .03 .02 /$ SR62 $04.02 .03 .03 /$ SR0 1 $04.02 .03 .03 /$ SR0 $404.02 .03 .03 /$ SR0 $504.02 .03 .03 /$ SR0 6 $04.02 .03 .03 /$ SR0 $704.02 .03 .03 /$ SR0 $804.02 .03 .03 /$ SR0 9 $04.02 .03 .03 /$ SR10 $04.02 .03 .03 /$ SR11 $04.02 .03 .03 /$ SR12 $04.02 .03 .03 /$ SR13 $04.02 .03 .03 /$ SR14 $04.02 .03 .03 /$ SR 15 $04.02 .03 .03 /$ SR16 $04.02 .03 .03 /$ SR17 $04.02 .03 .03 /$ SR 18 $04.02 .04 .01 /$ SR2 $704.02 .04 .01 /$ SR33 $04.02 .04 .01 /$ SR 49 $04.02 .04 .02 /$ SR25 $04.02 .04 .02 /$ SR2 8 05.01.02/SR01 $09.02 .01 /$ SR01 $09.02 .02 /$ SR01 09.02.02/SR02 09.02.02/SR0 $809.02 .03 /$ SR0 4 $09.02 .10 /$ SR01 $09.02 .11 /$ SR03 $11.02 /$ SR02

\#F 270 <inquire interior facilities>: $04.02 .05 .01 /$ SR0 $84.02 .05 .01 /$ SR0 $904.02 .05 .01 / S R 20$ $04.02 .05 .01 /$ SR21 $04.02 .05 .02 /$ SR20 $04.02 .05 .02 / S R 22$ $04.02 .05 .02 /$ SR2 $604.02 .05 .02 /$ SR2 $704.02 .05 .02 /$ SR2 9 $04.02 .05 .02 /$ SR31 $04.02 .05 .02 /$ SR32 $04.02 .05 .02 / S R 33$ $04.02 .05 .02 / S R 3404.02 .05 .02 / S R 42 \quad 04.02 .05 .02 / S R 43$ $04.02 .05 .02 / \mathrm{SR} 4404.02 .05 .02 / \mathrm{SR} 4504.02 .05 .02 / \mathrm{SR} 46$ 
$04.02 .05 .02 / S R 47 \quad 05.01 .02 / S R 01 \quad 09.02 .01 / S R 01 \quad 09.02 .02 / S R 01$ 09.02.02/SR02 09.02.02/SR05 09.02.02/SR08 09.02.10/SR03 $09.02 .11 / \mathrm{SR} 01$ 09.02.11/SR02 11.02/SR02

\#F 271 <inquire predefined interior representation>: $04.02 .05 .02 /$ SR21 $04.02 .05 .02 /$ SR22 $04.02 .05 .02 /$ SR23 $04.02 .05 .02 / S R 2404.02 .05 .02 / S R 2504.02 .05 .02 / S R 27$ $04.02 .05 .02 /$ SR2 $804.02 .05 .02 /$ SR2 $904.02 .05 .02 /$ SR30 $04.02 .05 .02 / S R 3105.01 .02 / S R 0109.02 .01 / S R 0109.02 .02 / S R 01$ $09.02 .02 /$ SR02 $09.02 .02 /$ SR05 $09.02 .02 /$ SR0 8 09.02.03/SR01 $09.02 .03 / \mathrm{SR} 0311.02 / \mathrm{SR} 02$

\#F 272 <inquire edge facilities>: $04.02 .05 .01 /$ SR42 $04.02 .05 .01 /$ SR43 $04.02 .05 .01 /$ SR5 1 $04.02 .05 .01 /$ SR52 $04.02 .05 .03 /$ SR19 $04.02 .05 .03 /$ SR2 1 $04.02 .05 .03 /$ SR2 $604.02 .05 .03 /$ SR2 $704.02 .05 .03 /$ SR32 $04.02 .05 .03 /$ SR33 $04.02 .05 .03 /$ SR34 $04.02 .05 .03 /$ SR35 $04.02 .05 .03 /$ SR36 $04.02 .05 .03 /$ SR3 $04.02 .05 .03 /$ SR 42 $04.02 .05 .03 / S R 4304.02 .05 .03 / S R 44 \quad 05.01 .02 / S R 0109.02 .01 / S R 01$ $09.02 .02 /$ SR01 $09.02 .02 /$ SR02 $09.02 .02 /$ SR0 $509.02 .02 /$ SR0 8 $09.02 .10 / \mathrm{SR} 0309.02 .11 / \mathrm{SR} 0109.02 .11 / \mathrm{SR} 0211.02 / \mathrm{SR} 02$

\#F 273 <inquire predefined edge representation>: $04.02 .05 .03 /$ SR20 $04.02 .05 .03 /$ SR21 $04.02 .05 .03 /$ SR22 $04.02 .05 .03 / S R 2304.02 .05 .03 / S R 24 \quad 04.02 .05 .03 / S R 25$ $04.02 .05 .03 /$ SR2 $64.02 .05 .03 /$ SR2 $704.02 .05 .03 / S R 28$ $04.02 .05 .03 /$ SR29 $05.01 .02 /$ SR01 $09.02 .01 /$ SR01 $09.02 .02 /$ SR01 $09.02 .02 /$ SR02 $09.02 .02 /$ SR0 $509.02 .02 /$ SR0 $809.02 .03 /$ SR0 1 $09.02 .03 /$ SR03 $11.02 /$ SR02

\#F 274 <inquire pattern facilities>: $04.02 .05 .02 /$ SR2 $804.02 .05 .02 /$ SR34 $04.02 .05 .04 /$ SR1 1 $04.02 .05 .04 /$ SR13 $04.02 .05 .04 /$ SR14 $05.01 .02 /$ SR01 $09.02 .01 /$ SR01 $09.02 .02 / \mathrm{SR} 0109.02 .02 / \mathrm{SR} 0209.02 .02 / \mathrm{SR} 0509.02 .02 / \mathrm{SR} 08$ $11.02 / \mathrm{SR} 02$

\#F 275 <inquire predefined pattern representation>: $04.02 .05 .04 /$ SR12 $04.02 .05 .04 /$ SR13 $04.02 .05 .04 /$ SR15 $04.02 .05 .04 /$ SR1 $604.02 .05 .04 /$ SR17 $04.02 .05 .04 /$ SR1 $805.01 .02 / S R 01$ 09.02.01/SR01 09.02.02/SR01 09.02.02/SR02 $09.02 .02 /$ SR0 5 $09.02 .02 / \mathrm{SR} 0809.02 .03 / \mathrm{SR} 03 \quad 09.02 .03 / \mathrm{SR} 0609.02 .10 / \mathrm{SR} 02$ $11.02 / \mathrm{SR} 02$

\#F 276 <inquire colour model facilities>:

$04.03 .02 .02 /$ SR15 $04.03 .02 .02 /$ SR16 $04.03 .02 .02 /$ SR17

$04.03 .02 .02 /$ SR18 $04.03 .02 .02 /$ SR19 $04.03 .02 .02 /$ SR2 0

$04.03 .02 .02 /$ SR22 $04.03 .02 .02 / S R 2304.03 .02 .02 / S R 24$

$04.03 .02 .02 /$ SR25 $04.03 .02 .02 /$ SR28 $04.03 .02 .02 / S R 2905.01 .02 / S R 01$ $09.02 .01 /$ SR01 $09.02 .02 /$ SR01 $09.02 .02 /$ SR0 $209.02 .02 /$ SR0 5 $09.02 .02 /$ SR0 $809.02 .10 /$ SR03 09.02.11/SR01 09.02.11/SR02 $11.02 / \mathrm{SR} 02$

\#F 277 <inquire colour facilities>: $04.02 .01 .02 /$ SR2 $804.02 .02 .02 / S R 2804.02 .03 .02 / S R 29$ 
$04.02 .05 .02 / \mathrm{SR} 30 \quad 04.02 .05 .03 / \mathrm{SR} 28 \quad 04.02 .05 .04 / \mathrm{SR} 18$ $04.03 .02 .02 /$ SR01 $04.03 .02 .02 /$ SR02 $04.03 .02 .02 /$ SR03 $04.03 .02 .02 / \mathrm{SR} 04 \quad 04.03 .02 .02 / \mathrm{SR} 05 \quad 04.03 .02 .02 / \mathrm{SR} 06$ $04.03 .02 .02 /$ SR07 $04.03 .02 .02 /$ SR0 8 04.03.02.02/SR10 $04.03 .02 .02 /$ SR11 $05.01 .02 /$ SR01 $09.02 .01 /$ SR01 $09.02 .02 / S R 01$ $09.02 .02 / \mathrm{SR} 02$ 09.02.02/SR05 09.02.02/SR08 $11.02 / \mathrm{SR} 02$

\#F 278 <inquire predefined colour representation>: $04.03 .02 .02 /$ SR09 $04.03 .02 .02 /$ SR10 $04.03 .02 .02 /$ SR1 1 $04.03 .02 .02 /$ SR12 $04.03 .02 .02 /$ SR13 $04.03 .02 .02 /$ SR1 4 $04.03 .02 .02 / S R 1505.01 .02 / S R 01 \quad 09.02 .01 / S R 0109.02 .02 / S R 01$ $09.02 .02 /$ SR02 $09.02 .02 /$ SR0 $509.02 .02 /$ SR0 $809.02 .03 /$ SR03 $09.02 .03 /$ SR0 $709.02 .10 /$ SR02 $11.02 /$ SR02

\#F 279 <inquire list of available generalized drawing primitives 3>: 04.01.08/SR04 05.01.02/SR01 09.02.01/SR01 09.02.02/SR01 09.02.02/SR02 09.02.02/SR05 09.02.02/SR08 09.02.10/SR03 $09.02 .11 /$ SR01 $09.02 .11 /$ SR02 $11.02 /$ SR02

\#F 280 <inquire list of available generalized drawing primitives>: 04.01.08/SR09 05.01.02/SR01 09.02.01/SR01 09.02.02/SR01 $09.02 .02 /$ SR02 $09.02 .02 /$ SR05 $09.02 .02 /$ SR0 $809.02 .10 /$ SR03 $09.02 .11 /$ SR01 $09.02 .11 /$ SR02 11.02/SR02

\#F 281 <inquire generalized drawing primitive 3>: $04.01 .08 /$ SR05 $05.01 .02 /$ SR01 09.02.01/SR01 $09.02 .02 /$ SR01 $09.02 .02 /$ SR02 09.02.02/SR05 09.02.02/SR08 $09.02 .02 /$ SR0 9 $11.02 /$ SR02

\#F 282 <inquire generalized drawing primitive>: $04.01 .08 /$ SR10 05.01.02/SR01 09.02.01/SR01 09.02.02/SR01 $09.02 .02 /$ SR02 09.02.02/SR05 09.02.02/SR08 $09.02 .02 /$ SR0 9 $11.02 / \mathrm{SR} 02$

\#F 283 <inquire list of available generalized structure elements>: 02.02.02/SR11 02.02.02/SR13 05.01.02/SR01 09.02.01/SR01 $09.02 .02 /$ SR01 $09.02 .02 /$ SR02 $09.02 .02 /$ SR0 $509.02 .02 /$ SR0 8 $09.02 .10 /$ SR03 09.02.11/SR01 09.02.11/SR02 $11.02 /$ SR02

\#F 284 <inquire number of display priorities supported>: 05.01.02/SR01 05.03/SR16 05.03/SR17 05.03/SR18 05.03/SR19 $09.02 .01 /$ SR01 $09.02 .02 /$ SR01 $09.02 .02 /$ SR02 $09.02 .02 /$ SR0 5 $09.02 .02 /$ SR0 $811.02 /$ SR02

\#F 285 <inquire workstation state table lengths>: $04.02 .01 .02 /$ SR17 $04.02 .01 .02 /$ SR18 $04.02 .02 .02 /$ SR17 $04.02 .02 .02 /$ SR18 $04.02 .03 .02 /$ SR17 $04.02 .03 .02 /$ SR1 8 $04.02 .05 .02 /$ SR18 $04.02 .05 .02 /$ SR19 $04.02 .05 .03 /$ SR1 7 $04.02 .05 .03 /$ SR18 $04.02 .05 .04 /$ SR0 8 04.02.05.04/SR0 9 $04.02 .05 .04 /$ SR10 $04.03 .02 .01 /$ SR0 $704.03 .02 .01 /$ SR0 $8 \quad 05.01 .02 / S R 01$ $06.02 .02 /$ SR12 $06.02 .02 /$ SR13 $09.02 .01 /$ SR01 $09.02 .02 /$ SR01 $09.02 .02 /$ SR02 $09.02 .02 /$ SR0 $509.02 .02 / S R 0811.02 /$ SR02

\#F 286 <inquire dynamics of structures>: 
05.01.02/SR01 $05.02 / S R 07 \quad 05.02 / S R 1905.02 / S R 2005.02 / S R 21$ $05.02 / S R 2405.02 / S R 2505.02 / S R 26 \quad 05.02 / S R 2705.02 / S R 2805.02 / S R 29$ $05.02 / S R 3105.02 / S R 3209.02 .01 / S R 0109.02 .02 / S R 0109.02 .02 / S R 02$ $09.02 .02 /$ SR05 $09.02 .02 /$ SR08 $11.02 /$ SR0 2

\#F 287 <inquire number of available logical input devices>:

05.01.02/SR01 09.02.01/SR01 09.02.02/SR01 09.02.02/SR02 $09.02 .02 /$ SR0 $711.02 /$ SR02

\#F 288 <inquire default locator device data 3>: 05.01.02/SR01 09.02.01/SR01 09.02.02/SR01 09.02.02/SR02 $09.02 .02 /$ SR0 $709.02 .10 /$ SR02 09.02.10/SR03 $11.02 /$ SR0 2

\#F 289 <inquire default locator device data>: 05.01.02/SR01 09.02.01/SR01 09.02.02/SR01 09.02.02/SR02 $09.02 .02 / \mathrm{SR} 0709.02 .10 / \mathrm{SR} 02 \quad 09.02 .10 / \mathrm{SR} 0311.02 / \mathrm{SR} 02$

\#F 290 <inquire default stroke device data 3>: 05.01.02/SR01 09.02.01/SR01 09.02.02/SR01 09.02.02/SR02 $09.02 .02 /$ SR0 $709.02 .10 /$ SR02 09.02.10/SR03 $11.02 /$ SR02

\#F 291 <inquire default stroke device data>:

05.01.02/SR01 09.02.01/SR01 09.02.02/SR01 09.02.02/SR02 $09.02 .02 /$ SR0 $709.02 .10 /$ SR02 09.02.10/SR03 $11.02 /$ SR0 2

\#F 292 <inquire default valuator device data 3>: $05.01 .02 /$ SR01 $09.02 .01 /$ SR01 $09.02 .02 /$ SR01 $09.02 .02 /$ SR02 $09.02 .02 /$ SR0 $709.02 .10 /$ SR02 09.02.10/SR03 $11.02 /$ SR0 2

\#F 293 <inquire default valuator device data>: 05.01.02/SR01 09.02.01/SR01 09.02.02/SR01 09.02.02/SR02 $09.02 .02 /$ SR0 $709.02 .10 /$ SR02 09.02.10/SR03 $11.02 / S R 02$

\#F 294 <inquire default choice device data 3>:

05.01.02/SR01 09.02.01/SR01 09.02.02/SR01 09.02.02/SR02 $09.02 .02 /$ SR0 $709.02 .10 /$ SR02 09.02.10/SR03 $11.02 /$ SR0 2

\#F 295 <inquire default choice device data>:

05.01.02/SR01 09.02.01/SR01 09.02.02/SR01 09.02.02/SR02 $09.02 .02 / \mathrm{SR} 0709.02 .10 / \mathrm{SR} 02$ 09.02.10/SR03 11.02/SR02

\#F 296 <inquire default pick device data 3>:

05.01.02/SR01 09.02.01/SR01 09.02.02/SR01 09.02.02/SR02 $09.02 .02 /$ SR07 09.02.10/SR02 09.02.10/SR03 $11.02 /$ SR02

\#F 297 <inquire default pick device data>:

05.01.02/SR01 09.02.01/SR01 09.02.02/SR01 09.02.02/SR02 $09.02 .02 /$ SR0 $709.02 .10 /$ SR02 09.02.10/SR03 $11.02 /$ SR0 2

\#F 298 <inquire default string device data 3>: 05.01.02/SR01 09.02.01/SR01 09.02.02/SR01 09.02.02/SR02 $09.02 .02 /$ SR0 $709.02 .10 /$ SR02 09.02.10/SR03 $11.02 /$ SR0 2

\#F 299 <inquire default string device data>: 
05.01.02/SR01 09.02.01/SR01 09.02.02/SR01 $09.02 .02 / S R 02$

$09.02 .02 /$ SR07 09.02.10/SR02 09.02.10/SR03 $11.02 /$ SR02

\#F 300 <inquire set of workstations to which posted>: 05.03/SR13 09.02.01/SR01 09.02.05/SR01 09.02.10/SR03 $09.02 .11 /$ SR01 09.02.11/SR02 11.02/SR02

\#F 301 <inquire open structure>:

$02.02 .01 /$ SR0 $502.02 .01 /$ SR0 $609.02 .01 /$ SR01 $11.02 /$ SR02

\#F 302 <inquire element pointer>:

$02.02 .01 /$ SR08 $09.02 .01 /$ SR03 $11.02 /$ SR02

\#F 303 <inquire current element type and size>:

02.02 .02 /SR02 02.02 .02 /SR05 $02.02 .02 /$ SR09 $02.03 .01 /$ SR0 1

$02.03 .01 /$ SR02 $04.01 .01 /$ SR02 $04.01 .01 /$ SR0 $504.01 .02 /$ SR0 2

$04.01 .02 /$ SR0 $504.01 .03 /$ SR02 $04.01 .03 /$ SR0 $504.01 .04 /$ SR0 2

$04.01 .04 /$ SR05 $04.01 .05 /$ SR02 $04.01 .05 /$ SR0 $504.01 .06 /$ SR0 2

$04.01 .06 /$ SR05 $04.01 .07 /$ SR02 $04.01 .07 /$ SR0 $704.01 .08 /$ SR0 2

$04.01 .08 /$ SR07 $04.02 .01 .01 /$ SR01 $04.02 .01 .02 /$ SR01 $04.02 .02 .01 / S R 01$

$04.02 .02 .02 /$ SR01 $04.02 .03 .01 /$ SR0 $904.02 .03 .02 / S R 01$

$04.02 .04 .01 /$ SR03 $04.02 .05 .01 /$ SR01 $04.02 .05 .02 /$ SR01

$04.02 .05 .03 /$ SR01 $04.03 .01 /$ SR02 $04.03 .03 /$ SR02 $04.03 .04 .01 /$ SR0 2

$04.03 .04 .01 /$ SR0 $506.01 .02 /$ SR02 $06.01 .02 /$ SR0 $506.01 .02 /$ SR10

$06.01 .02 /$ SR13 $06.01 .02 /$ SR24 $06.01 .02 /$ SR2 $706.01 .02 /$ SR30

$06.01 .02 /$ SR33 06.02.02/SR02 09.02.01/SR03 $11.02 /$ SR02

\#F 304 <inquire current element content>:

$02.02 .02 /$ SR0 $302.02 .02 /$ SR0 $602.02 .02 /$ SR10 $02.03 .01 /$ SR0 3

$04.01 .01 /$ SR03 $04.01 .01 /$ SR0 $604.01 .02 /$ SR03 $04.01 .02 /$ SR0 6

$04.01 .03 /$ SR03 $04.01 .03 /$ SR0 $604.01 .04 /$ SR03 $04.01 .04 /$ SR0 6

$04.01 .05 /$ SR03 $04.01 .05 /$ SR0 $604.01 .06 /$ SR03 $04.01 .06 /$ SR0 6

$04.01 .07 /$ SR03 $04.01 .07 /$ SR0 $804.01 .08 /$ SR03 $04.01 .08 /$ SR0 8

$04.02 .01 .01 /$ SR02 $04.02 .01 .02 /$ SR02 $04.02 .02 .01 /$ SR0 2

$04.02 .02 .02 / S R 0204.02 .03 .01 / S R 1004.02 .03 .02 / S R 02$

$04.02 .04 .01 /$ SR0 $404.02 .05 .01 /$ SR02 $04.02 .05 .02 /$ SR02

$04.02 .05 .03 /$ SR02 $04.03 .01 /$ SR03 $04.03 .03 /$ SR03 $04.03 .04 .01 / \mathrm{SR} 03$

$04.03 .04 .01 /$ SR0 $606.01 .02 /$ SR03 $06.01 .02 /$ SR0 $606.01 .02 /$ SR11

$06.01 .02 /$ SR14 $06.01 .02 /$ SR25 $06.01 .02 /$ SR2 $8 \quad 06.01 .02 /$ SR 31

$06.01 .02 /$ SR3 $406.02 .02 /$ SR03 $09.02 .01 /$ SR03 $09.02 .10 /$ SR02

11.02/SR02

\#F 305 <inquire element type and size>:

$02.03 .01 /$ SR0 $402.03 .01 /$ SR05 $09.02 .01 /$ SR01 $09.02 .05 /$ SR01

$09.02 .05 /$ SR02 $11.02 / \mathrm{SR} 02$

\#F 306 <inquire element content>:

$02.03 .01 /$ SR0 $609.02 .01 /$ SR01 $09.02 .05 /$ SR01 $09.02 .05 /$ SR02

$09.02 .10 /$ SR02 $11.02 /$ SR02

\#F 307 <inquire structure status>:

$02.02 .01 /$ SR0 $709.02 .01 /$ SR01 $11.02 /$ SR0 2

\#F 308 <inquire paths to ancestors>: 
$02.01 /$ SR03 02.01/SR05 $02.01 / S R 0602.01 / S R 0702.01 / S R 0902.01 / S R 10$ $02.01 /$ SR13 09.02.01/SR01 09.02.05/SR01 09.02.05/SR05 $09.02 .10 /$ SR01 09.02.10/SR02 09.02.10/SR03 09.02.11/SR03 $11.02 / \mathrm{SR} 02$

\#F 309 <inquire paths to descendants>:

$02.01 / S R 0402.01 / S R 0502.01 / S R 0602.01 / S R 0802.01 / S R 1102.01 / S R 12$ $02.01 /$ SR14 09.02.01/SR01 09.02.05/SR01 09.02.05/SR05

$09.02 .10 /$ SR01 09.02.10/SR02 09.02.10/SR03 09.02.11/SR03 $11.02 / \mathrm{SR} 02$

\#F 310 <element search>:

$02.03 .02 /$ SR01 $02.03 .02 /$ SR02 $02.03 .02 /$ SR0 $302.03 .02 / S R 04$ $02.03 .02 /$ SR0 $502.03 .02 /$ SR0 $602.03 .02 /$ SR0 $02.03 .02 /$ SR0 8 $02.03 .02 /$ SR09 09.02.01/SR01 09.02.05/SR01 09.02.10/SR01 $09.02 .10 /$ SR02 09.02.10/SR0 $409.02 .11 /$ SR03 $11.02 / S R 02$

\#F 311 <incremental spatial search 3>: $02.03 .03 /$ SR0 $102.03 .03 /$ SR02 $02.03 .03 /$ SR03 $02.03 .03 /$ SR0 4 $02.03 .03 /$ SR0 $502.03 .03 /$ SR0 $602.03 .03 /$ SR0 $702.03 .03 /$ SR0 8 $02.03 .03 /$ SR09 $02.03 .03 /$ SR10 $02.03 .03 /$ SR11 $02.03 .03 /$ SR12 $02.03 .03 /$ SR13 $02.03 .03 /$ SR14 $02.03 .03 /$ SR15 $02.03 .03 /$ SR1 6 $02.03 .03 /$ SR17 $02.03 .03 /$ SR18 $02.03 .03 /$ SR19 $02.03 .03 /$ SR20 $02.03 .03 /$ SR22 $06.01 .02 /$ SR19 $06.01 .02 /$ SR50 $09.02 .01 /$ SR0 1 $09.02 .05 /$ SR03 09.02.05/SR04 09.02.10/SR01 09.02.10/SR02 $09.02 .10 /$ SR0 $409.02 .10 /$ SR05 09.02.11/SR01 09.02.11/SR02 $09.02 .11 /$ SR03 $11.02 /$ SR02

\#F 312 <incremental spatial search>:

$02.03 .03 /$ SR01 $02.03 .03 /$ SR02 $02.03 .03 /$ SR0 $302.03 .03 /$ SR0 4 $02.03 .03 /$ SR0 $502.03 .03 /$ SR0 $602.03 .03 /$ SR0 $02.03 .03 /$ SR0 8 $02.03 .03 /$ SR09 $02.03 .03 /$ SR10 $02.03 .03 /$ SR11 $02.03 .03 /$ SR12 $02.03 .03 /$ SR13 $02.03 .03 /$ SR14 $02.03 .03 /$ SR15 $02.03 .03 /$ SR1 6 $02.03 .03 / \mathrm{SR} 1702.03 .03 / \mathrm{SR} 1802.03 .03 / \mathrm{SR} 1902.03 .03 / \mathrm{SR} 20$ $02.03 .03 /$ SR22 $06.01 .02 /$ SR19 $06.01 .02 /$ SR50 $09.02 .01 /$ SR0 1 $09.02 .05 /$ SR03 09.02.05/SR0 $409.02 .10 /$ SR0 $109.02 .10 /$ SR0 2 $09.02 .10 /$ SR0 $409.02 .10 /$ SR05 09.02.11/SR01 09.02.11/SR02 $09.02 .11 /$ SR03 $11.02 / S R 02$

\#F 313 <inquire input queue overflow>: $09.02 .01 /$ SR02 $11.02 /$ SR02

\#F 314 <inquire error handling mode>: $09.01 /$ SR03 09.02.01/SR01 11.02/SR02

\#F 315 <emergency close phigs>:

$09.01 /$ SR11 $11.02 /$ SR02

\#F 316 <error handling>:

09.01/SR04 09.01/SR05 09.01/SR06 09.01/SR07 09.01/SR08 09.01/SR10 $11.02 / \mathrm{SR} 02$

\#F 317 <error logging>:

$09.01 /$ SR0 $809.01 /$ SR09 $11.02 /$ SR02 
\#F 318 <set error handling mode>:

09.01/SR01 09.01.01.01/SR02 09.01.01.02/SR02 09.01.10.01/SR01 09.01.10.02/SR01 09.01.11.01/SR04 09.01.11.02/SR01 11.02/SR02

\#F 319 <escape>:

09.01.01.01/SR02 09.01.01.02/SR02 11.02/SR02

\#F 320 <pack data record>:

11.01/SR01

\#F 321 <unpack data record>:

$11.01 / \mathrm{SR} 02$

\#F 322 <create store>:

$11.02 /$ SR02 $11.02 /$ SR13

\#F 323 <delete store>:

$11.02 /$ SR02 $11.02 /$ SR14

\#F 324 <set error handler>:

09.01.11.01/SR01 09.01.11.01/SR02 09.01.11.01/SR03 11.02/SR02 
APPENDIX J

DATA STRUCTURE CROSS-REFERENCE

\#D 1.1:

01/SR01 01/SR02 01/SR03 09.01/SR11 09.01.01.01/SR01

09.01.01.01/SR02 09.01.01.01/SR03 09.01.01.01/SR0 4

09.01.01.01/SR05 09.01.01.01/SR06 09.01.01.01/SR07

09.01.01.02/SR01 09.01.01.02/SR02 09.01.01.02/SR03

09.01.01.02/SR04 09.01.01.02/SR05 09.01.01.02/SR06

09.01.01.02/SR07 09.02.01/SR01 09.02.01/SR02 09.02.01/SR03

$09.02 .01 / \mathrm{SR} 04$

\#D $1.2:$

05.01.01/SR02 05.01.01/SR03 05.01.01/SR04 05.01.01/SR05

$09.01 /$ SR11 09.01.01.01/SR01 09.01.01.01/SR03 09.01.01.01/SR04

09.01.01.02/SR01 09.01.01.02/SR03 09.01.01.02/SR0 4

$09.01 .02 .01 /$ SR01 $09.01 .02 .01 /$ SR02 $09.01 .02 .01 /$ SR0 3

$09.01 .02 .01 /$ SR0 $409.01 .02 .01 /$ SR0 $509.01 .02 .01 /$ SR12

$09.01 .02 .02 /$ SR01 $09.01 .02 .02 /$ SR02 $09.01 .02 .02 /$ SR0 3

$09.01 .02 .02 /$ SR0 $409.01 .02 .02 /$ SR0 $509.01 .02 .02 /$ SR12 09.02.01/SR02

\#D $1.3:$

$02.01 .03 .01 /$ SR01 $02.01 .03 .01 /$ SR03 $02.01 .03 .03 /$ SR01

$02.01 .03 .03 /$ SR03 $02.02 .01 /$ SR09 $09.01 /$ SR11 $09.01 .01 .01 /$ SR01

$09.01 .01 .01 /$ SR0 $409.01 .01 .01 /$ SR05 $09.01 .01 .01 / S R 06$

09.01.01.02/SR01 09.01.01.02/SR04 09.01.01.02/SR05

$09.01 .01 .02 /$ SR0 $609.02 .01 /$ SR03

\#D $1.4:$

03.01/SR03 03.01/SR05 03.01/SR08 $03.01 /$ SR10 09.01/SR11

09.01.01.01/SR01 09.01.01.01/SR04 09.01.01.01/SR07

09.01.01.02/SR01 09.01.01.02/SR04 09.01.01.02/SR07 09.02.01/SR04

\#D 2.1 :

$05.01 .02 /$ SR07 $09.02 .02 /$ SR01 $09.02 .02 /$ SR02

\#D 2.2 :

02.02.02/SR11 $04.02 .03 .01 /$ SR19 $04.02 .04 .01 /$ SR12 $05.01 .02 /$ SR0 5

$05.01 .02 /$ SR0 $609.01 .02 .01 /$ SR02 $09.01 .02 .02 /$ SR02 $09.02 .02 /$ SR01

$09.02 .02 /$ SR02 


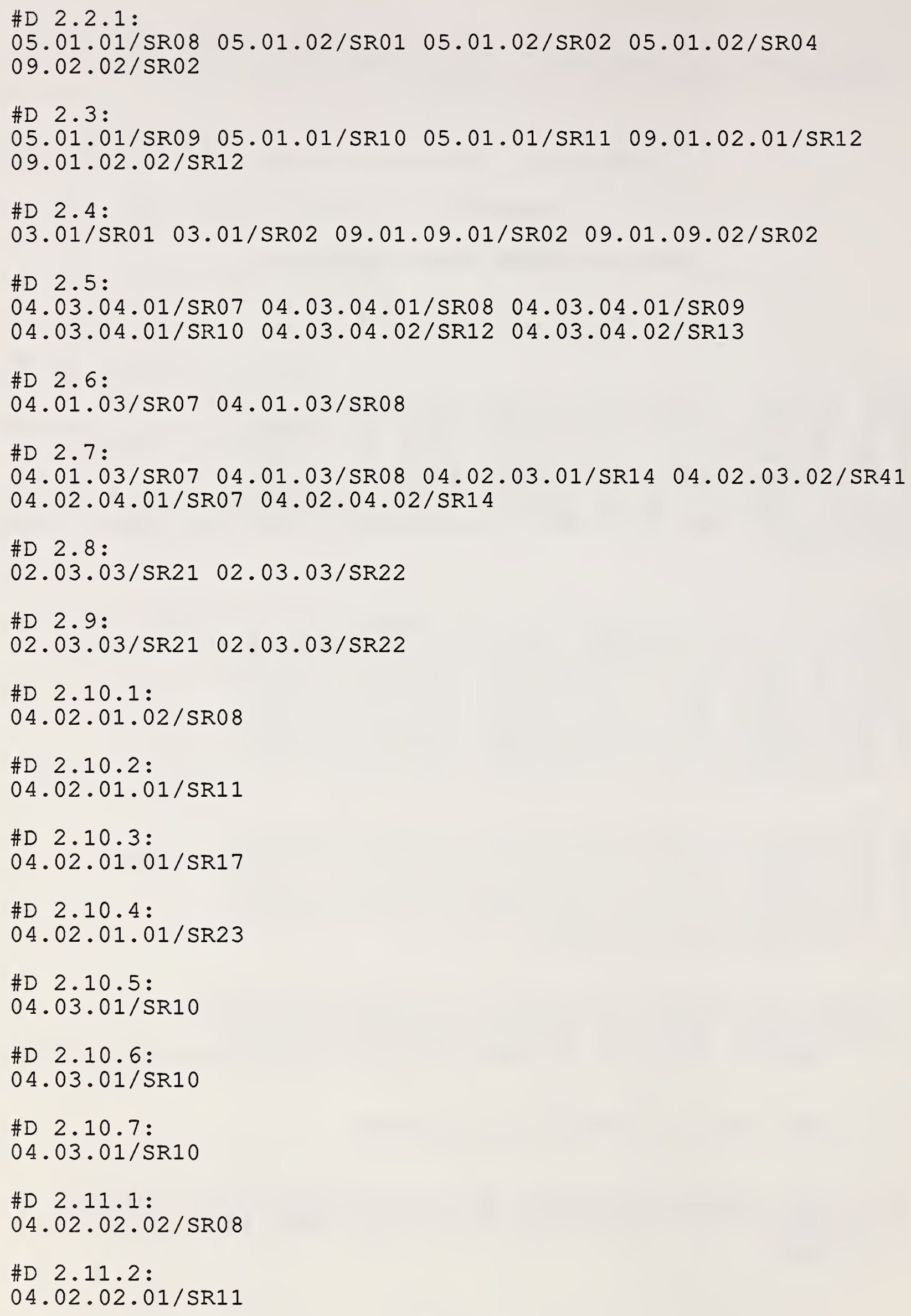


\#D $2.11 .3:$

$04.02 .02 .01 /$ SR1 8

\#D 2.11.4:

$04.02 .02 .01 / \mathrm{SR} 24$

\#D 2.11.5:

$04.03 .01 /$ SR 10

\#D 2.11 .6 :

$04.03 .01 /$ SR 10

\#D 2.11.7:

$04.03 .01 /$ SR10

\#D 2.12.1:

$04.02 .03 .02 /$ SR0 $804.02 .04 .02 /$ SR0 6

\#D 2.12.2:

$04.02 .03 .01 /$ SR23 $04.02 .04 .01 /$ SR1 6

\#D 2.12.3:

$04.02 .03 .01 /$ SR32 $04.02 .04 .01 /$ SR2 4

\#D $2.12 .4:$

$04.02 .03 .01 /$ SR3 $804.02 .04 .01 /$ SR30

\#D $2.12 .5:$

$04.02 .03 .01 / \mathrm{SR} 4404.02 .04 .01 / \mathrm{SR} 35$

\#D 2.12.6:

$04.02 .03 .01 /$ SR50 $04.02 .04 .01 /$ SR 40

\#D 2.12.7:

$04.03 .01 /$ SR10

\#D $2.12 .8:$

$04.03 .01 /$ SR10

\#D 2.12.9:

$04.03 .01 /$ SR10

\#D $2.12 .10:$

$04.03 .01 /$ SR10

\#D 2.12.11:

$04.03 .01 /$ SR 10

\#D 2.12.12:

$04.02 .03 .01 / \mathrm{SR} 62$

\#D 2.12.13:

$04.02 .03 .01 /$ SR8 2 


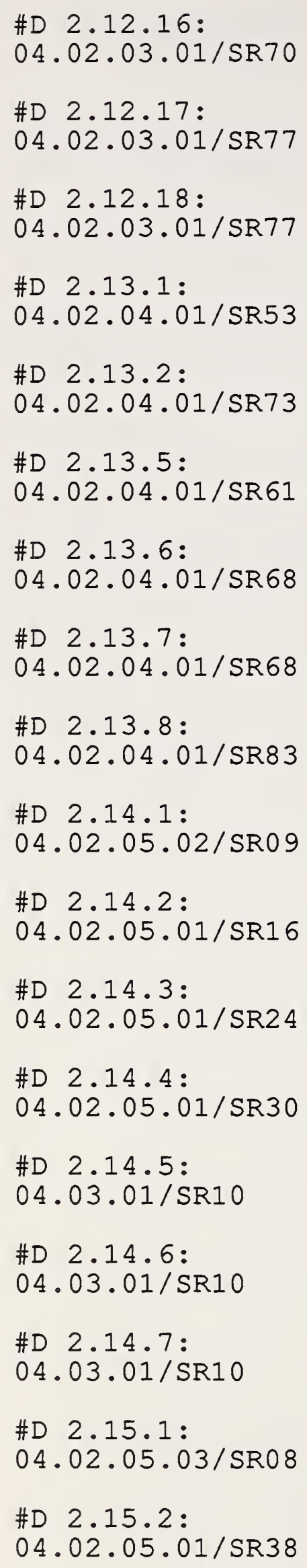


\#D $2.15 .3:$

$04.02 .05 .01 /$ SR 47

\#D 2.15 .4 :

$04.02 .05 .01 / \mathrm{SR} 53$

\#D 2.15 .5 :

$04.02 .05 .01 /$ SR59

\#D 2.15 .6 :

$04.03 .01 /$ SR10

\#D 2.15.7:

$04.03 .01 /$ SR10

\#D 2.15 .8 :

$04.03 .01 /$ SR 10

\#D 2.15.9:

$04.03 .01 / \mathrm{SR} 10$

\#D 2.16.1:

$04.02 .05 .01 /$ SR 64

\#D 2.16 .2 :

$04.02 .05 .01 / \mathrm{SR} 80$

\#D 2.16.3:

$04.02 .05 .01 /$ SR8 2

\#D 2.17 :

$02.02 .02 /$ SR12

\#D 2.18 :

02.02.02/SR11 02.02.02/SR12

\#D 2.22:

$04.03 .04 .01 /$ SR11

\#D 2.23.1:

$06.01 .02 /$ SR19

\#D 2.23 .2 :

$06.01 .02 /$ SR19

\#D 2.23 .3 :

$06.01 .02 /$ SR 50

\#D 2.23 .4 :

$06.01 .02 /$ SR5 4

\#D 2.23.5:

$06.01 .02 /$ SR35 $06.01 .02 / S R 3706.01 .02 / S R 47$ 
\#D 2.23.6:

$06.01 .02 /$ SR35 $06.01 .02 /$ SR36

\#D $2.23 .7:$

$06.01 .02 / \mathrm{SR} 3506.01 .02 / \mathrm{SR} 36 \quad 06.01 .02 / \mathrm{SR} 44 \quad 06.01 .02 / \mathrm{SR} 45$

$06.01 .02 / \mathrm{SR} 46$

\#D 3:

$02.03 .03 /$ SR02 $02.03 .03 /$ SR03 $02.03 .03 /$ SR0 $402.03 .03 /$ SR0 5

$02.03 .03 /$ SR0 6

\#D 3.1:

$04.03 .01 /$ SR0 $84.03 .01 /$ SR09

\#D 3.1.1:

$04.02 .01 .02 /$ SR03 $04.02 .01 .02 /$ SR0 $504.02 .01 .02 /$ SR0 6

$04.02 .01 .02 /$ SR07 $04.02 .01 .02 /$ SR0 8 04.02.01.02/SR0 9

$04.02 .01 .02 /$ SR $3504.02 .01 .02 /$ SR $4304.02 .01 .02 /$ SR 47

\#D $3.1 .2:$

$04.02 .01 .01 / \mathrm{SR} 0304.02 .01 .01 / \mathrm{SR} 0504.02 .01 .01 / \mathrm{SR} 06$

$04.02 .01 .01 /$ SR07 $04.02 .01 .01 /$ SR11 $04.02 .01 .01 / S R 12$

\#D $3.1 .3:$

$04.02 .01 .01 /$ SR03 $04.02 .01 .01 /$ SR14 $04.02 .01 .01 / \mathrm{SR} 15$

$04.02 .01 .01 /$ SR16 $04.02 .01 .01 /$ SR17 $04.02 .01 .01 /$ SR1 8

\#D $3.1 .4:$

$04.02 .01 .01 / \mathrm{SR} 0304.02 .01 .01 / \mathrm{SR} 20 \quad 04.02 .01 .01 / \mathrm{SR} 21$

$04.02 .01 .01 / \mathrm{SR} 2204.02 .01 .01 / \mathrm{SR} 2304.02 .01 .01 / \mathrm{SR} 24$

\#D $3.1 .5:$

$04.02 .01 .01 /$ SR0 $64.02 .01 .01 /$ SR07 $04.02 .01 .02 / S R 3504.03 .01 / S R 06$ $04.03 .01 /$ SR07 $04.03 .01 /$ SR10 $04.03 .01 /$ SR 11

\#D $3.1 .6:$

$04.02 .01 .01 / S R 1504.02 .01 .01 / S R 16 \quad 04.02 .01 .02 / S R 43 \quad 04.03 .01 / S R 06$ $04.03 .01 /$ SR07 $04.03 .01 /$ SR $10 \quad 04.03 .01 /$ SR 11

\#D $3.1 .7:$

04.02.01.01/SR21 04.02.01.01/SR22 $04.02 .01 .02 / S R 4704.03 .01 / S R 06$

$04.03 .01 /$ SR07 $04.03 .01 /$ SR10 $04.03 .01 /$ SR 11

\#D 3.2:

$04.03 .01 /$ SR08 $04.03 .01 /$ SR09

\#D $3.2 .1:$

$04.02 .02 .02 /$ SR03 $04.02 .02 .02 /$ SR0 $504.02 .02 .02 /$ SR0 6

$04.02 .02 .02 /$ SR07 $04.02 .02 .02 /$ SR0 8 04.02.02.02/SR0 9

$04.02 .02 .02 / S R 3504.02 .02 .02 / S R 43 \quad 04.02 .02 .02 / S R 48$

\#D $3.2 .2:$

$04.02 .02 .01 /$ SR03 $04.02 .02 .01 /$ SR0 $504.02 .02 .01 /$ SR0 6 
$04.02 .02 .01 /$ SR07 $04.02 .02 .01 /$ SR11 $04.02 .02 .01 /$ SR 12

\#D $3.2 .3:$

$04.02 .02 .01 /$ SR03 $04.02 .02 .01 /$ SR14 $04.02 .02 .01 /$ SR1 5

$04.02 .02 .01 /$ SR16 $04.02 .02 .01 /$ SR18 $04.02 .02 .01 /$ SR19

\#D $3.2 .4:$

$04.02 .02 .01 /$ SR03 $04.02 .02 .01 /$ SR21 $04.02 .02 .01 /$ SR2 2

$04.02 .02 .01 / \mathrm{SR} 23 \quad 04.02 .02 .01 / \mathrm{SR} 24 \quad 04.02 .02 .01 / \mathrm{SR} 25$

\#D $3.2 .5:$

$04.02 .02 .01 /$ SR0 $604.02 .02 .01 /$ SR0 $0404.02 .02 .02 /$ SR35 $04.03 .01 /$ SR0 6 $04.03 .01 /$ SR0 $04.03 .01 /$ SR10 $04.03 .01 /$ SR11

\#D 3.2 .6 :

$04.02 .02 .01 /$ SR15 $04.02 .02 .01 /$ SR1 $6 \quad 04.02 .02 .02 / S R 43 \quad 04.03 .01 /$ SR0 6 $04.03 .01 /$ SR07 $04.03 .01 /$ SR10 $04.03 .01 /$ SR 11

\#D 3.2 .7 :

$04.02 .02 .01 / \mathrm{SR} 2204.02 .02 .01 / \mathrm{SR} 23 \quad 04.02 .02 .02 / \mathrm{SR} 48 \quad 04.03 .01 / \mathrm{SR} 06$ $04.03 .01 /$ SR07 $04.03 .01 /$ SR10 $04.03 .01 /$ SR 11

\#D $3.3:$

$04.03 .01 /$ SR0 $804.03 .01 /$ SR09

\#D $3.3 .1:$

$04.02 .03 .02 / \mathrm{SR} 03$

$04.02 .03 .02 /$ SR 07

$04.02 .03 .02 /$ SR 38

$04.02 .03 .02 /$ SR61

$04.02 .04 .02 /$ SR0 3

$04.02 .04 .02 /$ SR0 6

$04.02 .03 .02 /$ SR0 5

$04.02 .03 .02 /$ SR0 6

$04.02 .03 .02 /$ SR0 $804.02 .03 .02 /$ SR0 9

$04.02 .03 .02 /$ SR39 $04.02 .03 .02 /$ SR5 7

$04.02 .03 .02 /$ SR64 $04.02 .04 .02 /$ SR0 1

$04.02 .04 .02 /$ SR0 $404.02 .04 .02 /$ SR0 5

$04.02 .04 .02 /$ SR 12

$04.02 .04 .02 / S R 0704.02 .04 .02 / S R 11$

$04.02 .04 .02 /$ SR2 9

$04.02 .04 .02 / S R 2404.02 .04 .02 / S R 27$

\#D $3.3 .2:$

$04.02 .03 .01 / S R 1104.02 .03 .01 / S R 1304.02 .03 .01 / S R 21$

$04.02 .03 .01 / \mathrm{SR} 2204.02 .03 .01 / \mathrm{SR} 2304.02 .03 .01 / \mathrm{SR} 24$

$04.02 .03 .01 /$ SR2 $804.02 .04 .01 /$ SR0 $504.02 .04 .01 /$ SR0 6

$04.02 .04 .01 /$ SR14 $04.02 .04 .01 /$ SR15 $04.02 .04 .01 /$ SR1 6

$04.02 .04 .01 /$ SR17 $04.02 .04 .01 /$ SR20

\#D $3.3 .3:$

$04.02 .03 .01 / S R 1104.02 .03 .01 / S R 21 \quad 04.02 .03 .01 / S R 22$

$04.02 .03 .01 /$ SR27 $04.02 .03 .01 /$ SR2 $804.02 .03 .01 /$ SR 32

$04.02 .03 .01 /$ SR33 $04.02 .04 .01 /$ SR0 $504.02 .04 .01 /$ SR1 4

$04.02 .04 .01 /$ SR15 $04.02 .04 .01 /$ SR19 $04.02 .04 .01 /$ SR 20

$04.02 .04 .01 / \mathrm{SR} 2404.02 .04 .01 / \mathrm{SR} 25$

\#D $3.3 .4:$

$04.02 .03 .01 /$ SR11 $04.02 .03 .01 /$ SR35 $04.02 .03 .01 /$ SR3 7

$04.02 .03 .01 /$ SR3 $84.02 .03 .01 /$ SR39 $04.02 .04 .01 /$ SR0 5

$04.02 .04 .01 /$ SR2 $604.02 .04 .01 /$ SR2 $904.02 .04 .01 /$ SR 30

$04.02 .04 .01 /$ SR31 
\#D $3.3 .5:$

$04.02 .03 .01 /$ SR11 $04.02 .03 .01 /$ SR4 $1 \quad 04.02 .03 .01 / S R 43$ $04.02 .03 .01 /$ SR4 $404.02 .03 .01 /$ SR $4504.02 .04 .01 /$ SR0 5 $04.02 .04 .01 /$ SR32 $04.02 .04 .01 /$ SR34 $04.02 .04 .01 /$ SR35 $04.02 .04 .01 / \mathrm{SR} 36$

\#D $3.3 .6:$

$04.02 .03 .01 /$ SR11 $04.02 .03 .01 /$ SR $4704.02 .03 .01 /$ SR 48 $04.02 .03 .01 /$ SR4 $904.02 .03 .01 /$ SR50 $04.02 .03 .01 /$ SR5 1 $04.02 .04 .01 /$ SR0 $504.02 .04 .01 /$ SR37 $04.02 .04 .01 / \mathrm{SR} 38$ $04.02 .04 .01 /$ SR39 $04.02 .04 .01 /$ SR40 $04.02 .04 .01 / \mathrm{SR} 41$

\#D $3.3 .7:$

$04.02 .03 .01 / S R 2104.02 .03 .01 / S R 2204.02 .03 .01 / S R 28$

$04.02 .03 .02 /$ SR38 $04.02 .04 .01 /$ SR14 $04.02 .04 .01 /$ SR 15

$04.02 .04 .01 /$ SR20 $04.02 .04 .02 /$ SR11 $04.03 .01 /$ SR0 $604.03 .01 /$ SR0 7 $04.03 .01 /$ SR10 $04.03 .01 /$ SR11

\#D $3.3 .8:$

$04.02 .03 .01 / \mathrm{SR} 2104.02 .03 .01 / \mathrm{SR} 2204.02 .03 .01 / \mathrm{SR} 28$

$04.02 .03 .02 /$ SR39 $04.02 .04 .01 /$ SR14 $04.02 .04 .01 /$ SR1 5

$04.02 .04 .01 / \mathrm{SR} 2004.02 .04 .02 / \mathrm{SR} 12 \quad 04.03 .01 / \mathrm{SR} 0604.03 .01 / \mathrm{SR} 07$ $04.03 .01 /$ SR10 $04.03 .01 /$ SR11

\#D $3.3 .9:$

$04.02 .03 .01 /$ SR37 $04.02 .03 .02 /$ SR5 $704.02 .04 .01 /$ SR2 9

$04.02 .04 .02 /$ SR2 $404.03 .01 /$ SR0 $604.03 .01 /$ SR0 $704.03 .01 /$ SR 10 $04.03 .01 /$ SR 11

\#D $3.3 .10:$

$04.02 .03 .01 / \mathrm{SR} 43 \quad 04.02 .03 .02 / \mathrm{SR} 61 \quad 04.02 .04 .01 / \mathrm{SR} 34$

$04.02 .04 .02 / \mathrm{SR} 2704.03 .01 / \mathrm{SR} 06 \quad 04.03 .01 / \mathrm{SR} 07 \quad 04.03 .01 / \mathrm{SR} 10$

$04.03 .01 /$ SR11

\#D $3.3 .11:$

$04.02 .03 .01 / S R 4804.02 .03 .01 / S R 4904.02 .03 .02 / S R 64$

$04.02 .04 .01 /$ SR3 $84.02 .04 .01 /$ SR39 $04.02 .04 .02 /$ SR29 $04.03 .01 /$ SR0 6 $04.03 .01 /$ SR07 $04.03 .01 /$ SR10 $04.03 .01 /$ SR11

\#D 3.3.12:

$02.03 .03 /$ SR15 $04.02 .03 .01 /$ SR03 $04.02 .03 .01 /$ SR11 $04.02 .03 .01 /$ SR5 8 $04.02 .03 .01 / \mathrm{SR} 6104.02 .03 .01 / \mathrm{SR} 6204.02 .03 .01 / \mathrm{SR} 63$

\#D 3.3.13:

$02.03 .03 / \mathrm{SR} 1504.02 .03 .01 / \mathrm{SR} 1104.02 .03 .01 / \mathrm{SR} 80 \quad 04.02 .03 .01 / \mathrm{SR} 82$ $04.02 .03 .01 / \mathrm{SR} 83$

\#D $3.3 .14:$

$02.03 .03 /$ SR15 $04.02 .03 .01 /$ SR0 6

\#D $3.3 .15:$

$02.03 .03 /$ SR15 
\#D $3.3 .16:$

$02.03 .03 / S R 1504.02 .03 .01 / S R 1104.02 .03 .01 / S R 6604.02 .03 .01 / S R 70$ $04.02 .03 .01 /$ SR71

\#D $3.3 .17:$

$02.03 .03 /$ SR15 $04.02 .03 .01 / S R 11 \quad 04.02 .03 .01 / S R 74 \quad 04.02 .03 .01 / S R 77$ $04.02 .03 .01 / S R 78$

\#D $3.3 .18:$

$02.03 .03 /$ SR15 $04.02 .03 .01 / S R 11 \quad 04.02 .03 .01 / S R 74 \quad 04.02 .03 .01 / S R 77$ $04.02 .03 .01 / S R 78$

\#D 3.4.1:

$04.02 .04 .01 /$ SR05 $04.02 .04 .01 /$ SR48 $04.02 .04 .01 /$ SR5 1

$04.02 .04 .01 /$ SR52 $04.02 .04 .01 /$ SR53 $04.02 .04 .01 /$ SR5 4

\#D $3.4 .2:$

$04.02 .04 .01 /$ SR0 $504.02 .04 .01 / S R 7104.02 .04 .01 / S R 73$

$04.02 .04 .01 / \operatorname{SR} 74$

\#D $3.4 .5:$

$04.02 .04 .01 /$ SR0 $504.02 .04 .01 /$ SR5 $04.02 .04 .01 / \mathrm{SR} 61$

$04.02 .04 .01 / \mathrm{SR} 62$

\#D $3.4 .6:$

$04.02 .04 .01 /$ SR05 $04.02 .04 .01 /$ SR65 $04.02 .04 .01 /$ SR 68

$04.02 .04 .01 /$ SR 69

\#D 3.4.7:

$04.02 .04 .01 /$ SR05 $04.02 .04 .01 /$ SR65 $04.02 .04 .01 /$ SR 68

$04.02 .04 .01 /$ SR 69

\#D 3.4.8:

$04.02 .04 .01 /$ SR05 $04.02 .04 .01 /$ SR76 $04.02 .04 .01 / S R 78$

$04.02 .04 .01 /$ SR79 $04.02 .04 .01 /$ SR83 $04.02 .04 .01 / S R 84$

\#D 3.5:

$04.03 .01 /$ SR08 $04.03 .01 /$ SR09

\#D 3.5.1:

$04.02 .05 .02 /$ SR0 $304.02 .05 .02 /$ SR0 $404.02 .05 .02 /$ SR0 6

$04.02 .05 .02 /$ SR0 $704.02 .05 .02 /$ SR0 $804.02 .05 .02 /$ SR0 9

$04.02 .05 .02 /$ SR10 $04.02 .05 .02 /$ SR35 $04.02 .05 .02 /$ SR3 9

$04.02 .05 .02 /$ SR51

\#D $3.5 .2:$

$04.02 .05 .01 /$ SR03 $04.02 .05 .01 /$ SR0 $404.02 .05 .01 / S R 07$

$04.02 .05 .01 /$ SR0 $84.02 .05 .01 /$ SR0 $904.02 .05 .01 / S R 10$

$04.02 .05 .01 /$ SR11 $04.02 .05 .01 /$ SR12 $04.02 .05 .01 /$ SR13

$04.02 .05 .01 /$ SR14 $04.02 .05 .01 /$ SR15 $04.02 .05 .01 /$ SR1 6 $04.02 .05 .01 /$ SR1 7

\#D $3.5 .3:$

$04.02 .05 .01 /$ SR03 $04.02 .05 .01 /$ SR0 $404.02 .05 .01 /$ SR1 4 
$04.02 .05 .01 /$ SR15 $04.02 .05 .01 /$ SR19 $04.02 .05 .01 /$ SR20 $04.02 .05 .01 / \mathrm{SR} 2104.02 .05 .01 / \mathrm{SR} 2404.02 .05 .01 / \mathrm{SR} 25$

\#D $3.5 .4:$

$04.02 .05 .01 /$ SR03 $04.02 .05 .01 /$ SR0 $404.02 .05 .01 /$ SR10 $04.02 .05 .01 /$ SR13 $04.02 .05 .01 /$ SR14 $04.02 .05 .01 /$ SR27 $04.02 .05 .01 / \mathrm{SR} 2804.02 .05 .01 / \mathrm{SR} 2904.02 .05 .01 / \mathrm{SR} 30$ $04.02 .05 .01 /$ SR31

\#D $3.5 .5:$

$04.02 .05 .01 /$ SR0 $804.02 .05 .01 /$ SR0 $904.02 .05 .02 /$ SR35 $04.03 .01 /$ SR0 6 $04.03 .01 / \mathrm{SR} 0704.03 .01 / \mathrm{SR} 1004.03 .01 / \mathrm{SR} 11$

\#D 3.5 .6 :

$04.02 .05 .01 /$ SR14 $04.02 .05 .01 /$ SR15 $04.02 .05 .01 /$ SR2 0 $04.02 .05 .01 / \mathrm{SR} 2104.02 .05 .02 / \mathrm{SR} 3904.03 .01 / \mathrm{SR} 0604.03 .01 / \mathrm{SR} 07$ $04.03 .01 /$ SR10 $04.03 .01 /$ SR11

\#D $3.5 .7:$

$04.02 .05 .01 / \mathrm{SR} 10 \quad 04.02 .05 .01 / \mathrm{SR} 13 \quad 04.02 .05 .01 / \mathrm{SR} 14$

$04.02 .05 .01 / S R 28 \quad 04.02 .05 .01 / S R 29 \quad 04.02 .05 .02 / S R 51 \quad 04.03 .01 / S R 06$ $04.03 .01 /$ SR07 $04.03 .01 /$ SR10 $04.03 .01 /$ SR 11

\#D $3.6:$

$04.03 .01 /$ SR08 $04.03 .01 /$ SR09

\#D $3.6 .1:$

$04.02 .05 .03 / S R 03 \quad 04.02 .05 .03 / S R 05 \quad 04.02 .05 .03 / S R 06$

$04.02 .05 .03 /$ SR0 $704.02 .05 .03 /$ SR0 $804.02 .05 .03 /$ SR0 9

$04.02 .05 .03 /$ SR30 $04.02 .05 .03 /$ SR3 $804.02 .05 .03 /$ SR 46

$04.02 .05 .03 /$ SR 50

\#D 3.6.2:

$04.02 .05 .01 /$ SR0 $404.02 .05 .01 /$ SR34 $04.02 .05 .01 /$ SR3 5

$04.02 .05 .01 /$ SR36 $04.02 .05 .01 /$ SR3 $804.02 .05 .01 /$ SR 39

\#D $3.6 .3:$

$04.02 .05 .01 / \mathrm{SR} 0404.02 .05 .01 / \mathrm{SR} 4104.02 .05 .01 / \mathrm{SR} 42$ $04.02 .05 .01 / \mathrm{SR} 4304.02 .05 .01 / \mathrm{SR} 4704.02 .05 .01 / \mathrm{SR} 48$

\#D $3.6 .4:$

$04.02 .05 .01 / \mathrm{SR} 04 \quad 04.02 .05 .01 / \mathrm{SR} 50 \quad 04.02 .05 .01 / \mathrm{SR} 51$

$04.02 .05 .01 /$ SR52 $04.02 .05 .01 /$ SR53 $04.02 .05 .01 /$ SR5 4

\#D 3.6.5:

$04.02 .05 .01 /$ SR0 $404.02 .05 .01 /$ SR5 $604.02 .05 .01 /$ SR5 7

$04.02 .05 .01 /$ SR58 $04.02 .05 .01 /$ SR59 $04.02 .05 .01 /$ SR 60

\#D 3.6 .6 :

$04.02 .05 .01 / \mathrm{SR} 3504.02 .05 .03 / \mathrm{SR} 30 \quad 04.03 .01 / \mathrm{SR} 06 \quad 04.03 .01 / \mathrm{SR} 07$ $04.03 .01 /$ SR10 $04.03 .01 /$ SR11

\#D 3.6.7:

$04.02 .05 .01 / S R 42 \quad 04.02 .05 .01 / S R 43 \quad 04.02 .05 .03 / S R 38 \quad 04.03 .01 / S R 06$ 
04.03.01/SR07 $04.03 .01 /$ SR10 $04.03 .01 /$ SR11

\#D 3.6.8:

$04.02 .05 .01 /$ SR51 $04.02 .05 .01 /$ SR52 $04.02 .05 .03 /$ SR $4604.03 .01 /$ SR0 6 $04.03 .01 /$ SR0 $04.03 .01 /$ SR10 $04.03 .01 /$ SR11

\#D 3.6.9:

$04.02 .05 .01 /$ SR57 $04.02 .05 .01 /$ SR58 $04.02 .05 .03 /$ SR50 $04.03 .01 /$ SR0 6 $04.03 .01 /$ SR0 $704.03 .01 /$ SR10 $04.03 .01 /$ SR11

\#D 3.7 :

$04.02 .05 .01 /$ SR79

\#D $3.7 .1:$

$04.02 .05 .01 /$ SR0 $304.02 .05 .01 /$ SR0 $404.02 .05 .01 /$ SR62

$04.02 .05 .01 /$ SR63 $04.02 .05 .01 /$ SR $6404.02 .05 .01 /$ SR 65

$04.02 .05 .01 /$ SR71 $04.02 .05 .01 /$ SR73 $04.02 .05 .01 /$ SR74

$04.02 .05 .01 /$ SR7 $604.02 .05 .01 /$ SR77

\#D 3.7.2:

$04.02 .05 .01 /$ SR0 $304.02 .05 .01 /$ SR04 $04.02 .05 .01 /$ SR68

$04.02 .05 .01 /$ SR69 $04.02 .05 .01 /$ SR70 $04.02 .05 .01 /$ SR71

$04.02 .05 .01 /$ SR72 $04.02 .05 .01 /$ SR73 $04.02 .05 .01 /$ SR74

$04.02 .05 .01 /$ SR7 $604.02 .05 .01 /$ SR77 $04.02 .05 .01 /$ SR 80

$04.02 .05 .01 /$ SR8 1

\#D 3.7.3:

$04.02 .05 .01 / \mathrm{SR} 0304.02 .05 .01 / \mathrm{SR} 0404.02 .05 .01 / \mathrm{SR} 68$

$04.02 .05 .01 /$ SR69 $04.02 .05 .01 /$ SR70 $04.02 .05 .01 / S R 71$

$04.02 .05 .01 /$ SR73 $04.02 .05 .01 /$ SR74 $04.02 .05 .01 / S R 76$

$04.02 .05 .01 /$ SR77 $04.02 .05 .01 /$ SR82 $04.02 .05 .01 /$ SR8 3

\#D 3.9 :

$04.01 /$ SR0 1

\#D 3.11 :

02.03.03/SR0 $602.03 .03 / S R 18 \quad 02.03 .03 / S R 1902.03 .03 / S R 20$

$04.03 .04 .01 / \mathrm{SR} 1104.03 .04 .01 / \mathrm{SR} 1204.03 .04 .01 / \mathrm{SR} 13$

$04.03 .04 .01 /$ SR1 $404.03 .04 .01 /$ SR15 $04.03 .04 .01 /$ SR1 6

$04.03 .04 .02 /$ SR0 $404.03 .04 .02 /$ SR0 8 04.03.04.02/SR0 9

\#D 3.12 :

$04.02 .05 .01 /$ SR79

\#D $3.12 .1:$

02.03.03/SR10 04.01/SR01 06.01.02/SR15 $06.01 .02 / S R 16$

$06.01 .02 / S R 1706.01 .02 / S R 1806.01 .02 / S R 1906.01 .02 / S R 20$

$06.01 .02 / S R 2106.01 .02 / S R 4206.01 .02 / S R 57$

\#D 3.12 .2 :

02.03.03/SR10 04.01/SR01 06.01.02/SR08 06.01.02/SR16

$06.01 .02 / S R 1706.01 .02 / S R 1806.01 .02 / S R 1906.01 .02 / S R 20$

$06.01 .02 / \mathrm{SR} 2206.01 .02 / \mathrm{SR} 4206.01 .02 / \mathrm{SR} 57$ 
\#D $3.12 .3:$

$02.03 .03 / S R 10 \quad 06.01 .02 / S R 43 \quad 06.01 .02 / S R 44 \quad 06.01 .02 / S R 45$

$06.01 .02 / S R 46 \quad 06.01 .02 / S R 47 \quad 06.01 .02 / S R 48 \quad 06.01 .02 / S R 50$

$06.01 .02 /$ SR51 $06.01 .02 /$ SR5 $606.01 .02 /$ SR57 $06.01 .02 /$ SR5 9

$06.01 .02 /$ SR60 06.01.02/SR61 06.01.02/SR62 06.01.02/SR63

$06.01 .02 /$ SR 64

\#D $3.12 .4:$

$06.01 .02 /$ SR53 06.01.02/SR54 06.01.02/SR55 06.01.02/SR58

$06.01 .02 /$ SR59 $06.01 .02 /$ SR60 $06.01 .02 /$ SR61 $06.01 .02 /$ SR62

$06.01 .02 /$ SR63 $06.01 .02 /$ SR64

\#D $4.1:$

05.01.01/SR01 05.01.01/SR04 05.01.01/SR05 05.01.01/SR0 6

$05.01 .01 /$ SR0 $05.01 .01 /$ SR11 $05.01 .01 /$ SR12 $09.01 /$ SR11

$09.02 .02 /$ SR03

\#D $4.1 .1:$

$09.01 .02 .01 / \mathrm{SR} 0309.01 .02 .02 / \mathrm{SR} 03 \quad 09.02 .02 / \mathrm{SR} 03$

\#D $4.2:$

$02.01 .02 .01 / \mathrm{SR} 05 \quad 02.01 .02 .02 / \mathrm{SR} 01 \quad 02.01 .02 .02 / \mathrm{SR} 02$

$02.01 .02 .02 /$ SR0 $52.01 .02 .02 /$ SR0 $6202.01 .02 .03 /$ SR0 2

$02.01 .03 .01 /$ SR01 $02.01 .03 .01 /$ SR03 $02.01 .03 .03 / S R 01$

$02.01 .03 .03 /$ SR0 $302.02 .01 / S R 0102.02 .01 / S R 0402.02 .01 / S R 05$

$02.02 .01 /$ SR0 $62.02 .01 /$ SR09 $02.03 .01 /$ SR01 $02.03 .01 /$ SR0 2

$02.03 .01 /$ SR03 $09.01 /$ SR11

\# $4.3:$

$02.01 .02 .01 /$ SR0 $602.01 .02 .01 /$ SR07 $02.01 .02 .02 / S R 11$

$02.01 .02 .02 /$ SR12 $02.01 .03 .01 /$ SR0 $402.01 .03 .01 /$ SR0 5

$02.01 .03 .01 /$ SR0 $602.01 .03 .03 /$ SR0 $402.01 .03 .03 /$ SR0 5

$02.01 .03 .03 /$ SR0 $602.02 .01 /$ SR02 $02.02 .01 /$ SR03 $02.02 .01 / \mathrm{SR} 08$

$02.02 .03 /$ SR02 $02.02 .03 /$ SR03 $02.02 .03 /$ SR0 $402.02 .03 /$ SR0 6

$02.02 .03 /$ SR0 $702.02 .03 /$ SR0 $802.02 .03 /$ SR09 $02.02 .03 /$ SR10

$02.02 .04 /$ SR01 $02.02 .04 /$ SR02 $02.02 .04 /$ SR0 $602.02 .04 /$ SR0 7

$02.02 .04 /$ SR11 $02.02 .05 /$ SR01 $02.02 .05 /$ SR02 $02.02 .05 /$ SR03

$02.03 .01 /$ SR01 $02.03 .01 /$ SR02 $02.03 .01 /$ SR03 $03.03 / S R 18$

$09.01 .05 .01 / \mathrm{SR} 0309.01 .05 .01 / \mathrm{SR} 0409.01 .05 .02 / \mathrm{SR} 03$

$09.01 .05 .02 /$ SR0 4

\#D $4.4:$

$02.02 .03 /$ SR01 $02.02 .03 /$ SR02 $02.02 .03 /$ SR03 $02.02 .03 /$ SR0 4

$02.02 .03 /$ SR05 $02.02 .03 /$ SR0 $602.02 .03 /$ SR 11

\#D $4.5:$

$02.01 .01 /$ SR01 $02.01 .01 /$ SR02 $02.01 .01 / S R 0302.01 .01 / S R 04$

$02.01 .01 /$ SR05 $02.01 .01 /$ SR0 $602.01 .01 /$ SR07 $02.01 .01 /$ SR0 8

02.01.01/SR10 02.01.02.01/SR01 02.01.02.01/SR03 02.01.02.01/SR05

$02.01 .02 .02 /$ SR01 $02.01 .02 .02 /$ SR02 $02.01 .02 .02 /$ SR03

$02.01 .02 .02 / \mathrm{SR0} 402.01 .02 .02 / \mathrm{SR} 05 \quad 02.01 .02 .02 / \mathrm{SR} 06$

$02.01 .02 .02 /$ SR0 $702.01 .02 .02 /$ SR0 $802.01 .02 .03 /$ SR01

$02.01 .02 .03 /$ SR02 $02.02 .01 /$ SR0 $03.04 / S R 0203.04 / S R 0303.04 / S R 04$

09.01.05.01/SR01 09.01.05.02/SR01 09.02.05/SR03 09.02.05/SR04 
$09.02 .05 /$ SR0 5

\#D 4.5.1:

02.02.01/SR05 02.02.01/SR06 09.01.05.01/SR01 09.01.05.02/SR01

$09.02 .05 /$ SR01

\#D 4.6 :

03.01/SR0 $403.01 /$ SR0 $603.01 / S R 0703.01 / S R 0903.01 / S R 1109.01 / S R 11$

\#D 4.6.1:

09.01.09.01/SR05 09.01.09.02/SR05 09.02.09/SR01

\#D 4.6 .2 :

09.01.09.01/SR03 09.01.09.02/SR03 09.02.09/SR01

\#D $4.7:$

03.02/SR01 03.02/SR02 03.02/SR03 03.03/SR0 $603.03 /$ SR0 $703.03 / S R 08$ $09.01 .09 .01 /$ SR0 $09.01 .09 .02 /$ SR0 6

\#D 4.8 :

03.02/SR01 03.02/SR02 03.02/SR03 03.03/SR14 03.03/SR15 03.03/SR16 03.03/SR17 09.01.09.01/SR06 09.01.09.02/SR06

\#D 4.9 .5 .1 :

$09.01 .03 .01 /$ SR13 $09.01 .03 .02 /$ SR13

\#D 4.9.6.1:

$09.01 .03 .01 /$ SR13 $09.01 .03 .02 /$ SR13

\#D 5.1:

05.01.01/SR06 05.01.01/SR12 09.01.02.01/SR03 09.01.02.02/SR03

\#D $5.2:$

$05.01 .02 / \mathrm{SR} 08$

\#D 5.3 :

05.01.01/SR08 $05.01 .02 / S R 0205.01 .02 / S R 08$

\#D 5.4 :

$05.02 / S R 10$

\#D $5.4 .1:$

06.02.02/SR08 09.01.04.01/SR01 09.01.04.02/SR01

\#D $5.4 .2:$

04.01/SR01 $06.02 .02 / S R 09 \quad 06.02 .02 / S R 10 \quad 06.02 .02 / S R 18$

$06.02 .02 / S R 1906.02 .02 / S R 2006.02 .02 / S R 2106.02 .02 / S R 22$

\#D 5.4 .2 .1 :

06.02.02/SR08 $06.02 .02 / S R 18 \quad 06.02 .02 / S R 1906.02 .02 / S R 20$

$06.02 .02 / S R 2106.02 .02 / S R 2209.01 .03 .01 / S R 0209.01 .03 .01 / S R 13$

$09.01 .03 .01 /$ SR1 $409.01 .03 .02 /$ SR02 $09.01 .03 .02 /$ SR13

$09.01 .03 .02 /$ SR14 $09.02 .03 /$ SR0 8 
\#D $5.4 .2 .2:$

$06.02 .02 / S R 1106.02 .02 / S R 24 \quad 06.02 .02 / S R 27 \quad 06.02 .02 / S R 28$ $06.02 .02 / S R 29$

\#D $5.4 .2 .3:$

$04.02 .05 .01 /$ SR79 $06.02 .02 /$ SR17 $06.02 .02 /$ SR25 $06.02 .02 / S R 26$

\#D $5.4 .2 \cdot 3 \cdot 1:$

06.02.01/SR01 06.02.01/SR02 09.02.04/SR05 09.02.04/SR0 6 $09.02 .04 / S R 07$

\# $5.4 .2 .3 .2:$

06.02.01/SR01 06.02.01/SR02 09.02.04/SR01 09.02.04/SR02

09.02.04/SR03 09.02.04/SR04 09.02.04/SR08 09.02.04/SR09

$09.02 .04 /$ SR10

\#D $5 \cdot 4 \cdot 2 \cdot 3 \cdot 3:$

$09.01 .04 .01 / \mathrm{SR} 0409.01 .04 .01 / \mathrm{SR} 0509.01 .04 .02 / \mathrm{SR} 04$

$09.01 .04 .02 /$ SR0 5

\#D $5.4 .2 .4:$

$06.02 .02 / \mathrm{SR} 1706.02 .02 / \mathrm{SR} 2506.02 .02 / \mathrm{SR} 26$

\#D $5.4 .2 .4 .1:$

$06.02 .01 /$ SR01 $06.02 .01 /$ SR02 $06.02 .02 /$ SR14 $06.02 .02 /$ SR15 $06.02 .02 /$ SR16 $06.02 .02 /$ SR23 $09.02 .04 /$ SR0 $509.02 .04 /$ SR0 6 $09.02 .04 /$ SR07

\#D $5.4 .2 .4 .2:$

$06.02 .01 /$ SR01 $06.02 .01 /$ SR02 $06.02 .02 / S R 1406.02 .02 /$ SR15 $06.02 .02 /$ SR16 $06.02 .02 /$ SR23 $09.02 .04 /$ SR01 $09.02 .04 /$ SR02 $09.02 .04 /$ SR03 $09.02 .04 /$ SR0 $409.02 .04 /$ SR0 $809.02 .04 /$ SR0 9 $09.02 .04 /$ SR 10

\#D $5.4 .2 .4 .3:$

$06.02 .02 / S R 1406.02 .02 / S R 15 \quad 06.02 .02 / S R 16 \quad 06.02 .02 / S R 23$

$09.01 .04 .01 / \mathrm{SR} 0409.01 .04 .01 / \mathrm{SR} 0509.01 .04 .02 / \mathrm{SR} 04$

$09.01 .04 .02 /$ SR0 5

\#D $5.4 .2 .4 .4:$

$06.02 .02 /$ SR14 $06.02 .02 /$ SR15 $06.02 .02 /$ SR23

\#D $5.4 .2 .4 .5:$

$06.02 .02 / S R 1406.02 .02 / S R 1506.02 .02 / S R 16 \quad 06.02 .02 / S R 23$

\#D $5.4 .2 .4 .6:$

$06.02 .02 / S R 1406.02 .02 / S R 1506.02 .02 / S R 16 \quad 06.02 .02 / S R 23$

\#D $5.5:$

$04.03 .03 /$ SR0 5

\#D $5.5 .1:$

$04.03 .03 /$ SR0 $604.03 .03 /$ SR07 $04.03 .03 /$ SR12 $04.03 .03 /$ SR15 
\#D $5.5 .2:$

$04.03 .03 /$ SR0 $604.03 .03 /$ SR0 $04.03 .03 /$ SR13

\#D $5.5 .3:$

$04.03 .03 / S R 0404.03 .03 / S R 14$

\#D 5.6 :

$04.01 /$ SR01 $05.02 / S R 1006.03 / S R 0506.03 / S R 1606.03 / S R 17$

\#D 5.6.1:

$06.03 /$ SR15 $06.03 /$ SR18 $06.03 /$ SR19 $06.03 /$ SR20

\#D $5.6 .2:$

$06.03 /$ SR01 $06.03 /$ SR0 $606.03 / S R 0706.03 / S R 0806.03 / S R 1206.03 / S R 13$ $06.03 /$ SR14 09.01.04.01/SR02 09.01.04.01/SR06 09.01.04.02/SR02 $09.01 .04 .02 /$ SR0 6

\#D $5.6 .3:$

$04.02 .05 .01 / S R 7906.03 / S R 01 \quad 06.03 / S R 08 \quad 06.03 / S R 1206.03 / S R 13$

$09.01 .04 .01 /$ SR02 $09.01 .04 .01 /$ SR0 $609.01 .04 .02 /$ SR0 2

$09.01 .04 .02 /$ SR0 6

\#D $5.6 .4:$

$06.03 /$ SR01 $06.03 / S R 0906.03 / S R 1006.03 / S R 1106.03 / S R 1206.03 / S R 13$ $06.03 /$ SR14 09.01.04.01/SR03 09.01.04.01/SR07 09.01.04.02/SR03 $09.01 .04 .02 / S R 07$

\#D $5.6 .5:$

$04.02 .05 .01 / S R 7906.03 / S R 01 \quad 06.03 / S R 1106.03 / S R 1206.03 / S R 13$ $09.01 .04 .01 /$ SR0 $309.01 .04 .01 /$ SR0 $09.01 .04 .02 /$ SR03

$09.01 .04 .02 / \mathrm{SR} 07$

\#D $5.7:$

$02.01 .02 .01 /$ SR0 $402.01 .02 .02 /$ SR10 $02.01 .03 .01 /$ SR01

$02.01 .03 .01 /$ SR03 $02.01 .03 .02 /$ SR0 $502.01 .03 .02 /$ SR0 6

$02.01 .03 .03 /$ SR0 $102.01 .03 .03 /$ SR03 $05.02 /$ SR $1005.03 /$ SR0 7

$05.03 /$ SR09 05.03/SRI0 05.03/SR11 05.03/SR14

\#D 5.7 .1 :

$05.03 /$ SR02 $05.03 /$ SR0 $605.03 /$ SR15

\#D $5.7 .2:$

$05.03 /$ SR03 05.03/SR0 $405.03 /$ SR0 $605.03 /$ SR15

\#D $5.8 .1:$

$04.03 .03 / S R 06 \quad 04.03 .03 / S R 07 \quad 05.02 / S R 01 \quad 05.02 / S R 03 \quad 05.02 / S R 04$ $05.02 /$ SR0 $805.02 / S R 2205.02 / S R 2305.02 / S R 2405.02 / S R 2505.02 / S R 26$ $05.02 / S R 2705.02 / S R 2805.02 / S R 29 \quad 05.02 / S R 3105.02 / S R 32$

\#D 5.8 .2 :

$04.03 .03 /$ SR0 $605.02 / S R 02 \quad 05.02 /$ SR03 $05.02 / S R 0405.02 /$ SR0 8

$05.02 /$ SR23 $05.02 /$ SR24 $05.02 /$ SR2 $505.02 / S R 2605.02 / S R 2705.02 / S R 28$ $05.02 /$ SR29 $05.02 /$ SR31 $05.02 /$ SR32 
\#D $5.8 .3:$

$04.03 .03 /$ SR0 $604.03 .03 /$ SR0 $705.02 / S R 04 \quad 05.02 / S R 09 \quad 05.02 / S R 11$ $05.02 /$ SR12

\#D $5.8 .4:$

05.02/SR0 $405.02 / S R 0905.02 / S R 1105.02 / S R 1205.02 / S R 1305.02 / S R 14$ $05.02 / S R 1505.02 / S R 16 \quad 05.02 / S R 17 \quad 05.02 / S R 18 \quad 05.02 / S R 2205.02 / S R 23$ $05.02 / S R 2405.02 / S R 2505.02 / S R 26 \quad 05.02 / S R 27 \quad 05.02 / S R 28 \quad 05.02 / S R 29$ $05.02 /$ SR31 $05.02 /$ SR32 $06.02 .02 /$ SR2 $706.02 .02 /$ SR2 $806.03 /$ SR 18 $06.03 /$ SR1 9

\#D $5.9:$

$04.02 .01 .02 /$ SR 18

\#D 5.9.1:

$04.02 .01 .02 /$ SR0 $604.02 .01 .02 /$ SR0 $7 \quad 04.02 .01 .02 / S R 10$ $04.02 .01 .02 /$ SR12 $04.02 .01 .02 /$ SR13

\#D $5.9 .2:$

$04.02 .01 .02 /$ SR0 $604.02 .01 .02 /$ SR0 $7 \quad 04.02 .01 .02 / S R 11$

$04.02 .01 .02 /$ SR1 $404.02 .01 .02 /$ SR15 $04.02 .01 .02 /$ SR1 6

$04.02 .01 .02 /$ SR2 $404.02 .01 .02 /$ SR2 $509.02 .03 /$ SR02

\#D 5.9.2.1:

$04.02 .01 .02 /$ SR10 $04.02 .01 .02 /$ SR12 $04.02 .01 .02 / S R 13$

$04.02 .01 .02 /$ SR3 $504.02 .01 .02 /$ SR $43 \quad 04.02 .01 .02 / S R 44$

$04.02 .01 .02 /$ SR $4504.02 .01 .02 / S R 47 \quad 04.02 .01 .02 / S R 48$

$04.02 .01 .02 /$ SR4 $909.01 .03 .01 /$ SR01 09.01.03.02/SR01

$09.02 .03 /$ SR01

\#D $5.9 .2 .2:$

$04.02 .01 .02 /$ SR33 $04.02 .01 .02 /$ SR3 $404.02 .01 .02 /$ SR35

\#D $5.9 \cdot 2 \cdot 3:$

$04.02 .01 .02 / S R 4104.02 .01 .02 / S R 42 \quad 04.02 .01 .02 / S R 43$ $04.02 .01 .02 / S R 4404.02 .01 .02 / S R 45$

\#D $5.9 .2 .4:$

$04.02 .01 .02 / S R 4604.02 .01 .02 / S R 47 \quad 04.02 .01 .02 / S R 48$

$04.02 .01 .02 /$ SR4 $909.01 .03 .01 /$ SR12 $09.01 .03 .02 /$ SR 12

\#D $5.10:$

$04.02 .02 .02 /$ SR 18

\#D $5.10 .1:$

$04.02 .02 .02 /$ SR0 $604.02 .02 .02 /$ SR0 $7 \quad 04.02 .02 .02 / S R 10$ $04.02 .02 .02 /$ SR12 $04.02 .02 .02 /$ SR13

\#D $5.10 .2:$

$04.02 .02 .02 /$ SR0 $604.02 .02 .02 /$ SR0 $704.02 .02 .02 / S R 11$

$04.02 .02 .02 /$ SR14 $04.02 .02 .02 /$ SR15 $04.02 .02 .02 /$ SR1 6

$04.02 .02 .02 / S R 2404.02 .02 .02 /$ SR2 $509.02 .03 /$ SR02

\#D $5.10 .2 .1:$

$04.02 .02 .02 /$ SR10 $04.02 .02 .02 /$ SR12 $04.02 .02 .02 / S R 13$ 
$04.02 .02 .02 / \mathrm{SR} 35$ $04.02 .02 .02 / \mathrm{SR} 45$ $04.02 .02 .02 / S R 50$

$04.02 .02 .02 / S R 43$ $04.02 .02 .02 / S R 48$ $09.01 .03 .01 /$ SR01
$04.02 .02 .02 / S R 44$ $04.02 .02 .02 / S R 49$ $09.01 .03 .02 /$ SR0 1 $09.02 .03 /$ SR0 1

\#D 5.10 .2 .2 :

$04.02 .02 .02 /$ SR33 $04.02 .02 .02 / S R 34 \quad 04.02 .02 .02 / S R 35$

\#D $5.10 \cdot 2 \cdot 3$ :

$04.02 .02 .02 / S R 4104.02 .02 .02 / S R 4204.02 .02 .02 / S R 43$ $04.02 .02 .02 / S R 4404.02 .02 .02 / S R 45$

\#D 5.10 .2 .4 :

$04.02 .02 .02 / S R 47 \quad 04.02 .02 .02 / S R 48 \quad 04.02 .02 .02 / S R 49$ $04.02 .02 .02 / S R 50 \quad 09.01 .03 .01 / S R 1209.01 .03 .02 / S R 12$

\#D 5.11 :

$04.02 .03 .02 /$ SR1 8

\#D 5.11 .1 :

$04.02 .03 .02 /$ SR0 6 $04.02 .03 .02 /$ SR12 $04.02 .03 .02 / S R 0704.02 .03 .02 / S R 10$ $04.02 .04 .02 /$ SR0 5 $04.02 .03 .02 /$ SR13 $04.02 .04 .02 /$ SR0 4 \#D 5.11 .2 :

$04.02 .03 .02 /$ SR0 $604.02 .03 .02 /$ SR0 $04.02 .03 .02 /$ SR1 1 $04.02 .03 .02 /$ SR14 $04.02 .03 .02 /$ SR15 $04.02 .03 .02 / S R 16$ $04.02 .03 .02 / S R 2404.02 .03 .02 / S R 2504.02 .04 .02 / S R 04$ $04.02 .04 .02 /$ SR0 $504.02 .04 .02 /$ SR09 $09.02 .03 /$ SR02

\#D $5.11 .2 .1:$

$04.02 .03 .02 /$ SR10 $04.02 .03 .02 /$ SR12 $04.02 .03 .02 /$ SR13 $04.02 .03 .02 /$ SR3 $84.02 .03 .02 /$ SR3 $9404.02 .03 .02 /$ SR5 7 $04.02 .03 .02 /$ SR61 $04.02 .03 .02 / S R 6404.02 .04 .02 /$ SR 11 $04.02 .04 .02 /$ SR12 $04.02 .04 .02 / S R 2404.02 .04 .02 / S R 27$ $04.02 .04 .02 / S R 2909.01 .03 .01 / S R 0109.01 .03 .02 / S R 01$

\#D 5.11 .2 .2 :

$04.02 .03 .02 /$ SR35 $04.02 .03 .02 / S R 3604.02 .03 .02 / S R 38$ $04.02 .03 .02 / S R 40 \quad 04.02 .04 .02 / S R 1104.02 .04 .02 / S R 13$

\#D 5.11 .2 .3 :

$04.02 .03 .02 /$ SR32 $04.02 .03 .02 /$ SR33 $04.02 .03 .02 /$ SR34 $04.02 .03 .02 /$ SR35 $04.02 .03 .02 /$ SR3 $04.02 .03 .02 /$ SR39 $04.02 .03 .02 /$ SR $4704.02 .04 .02 /$ SR10 $04.02 .04 .02 /$ SR12 $04.02 .04 .02 / S R 20$

\#D 5.11 .2 .4 :

$04.02 .03 .02 /$ SR5 $604.02 .03 .02 / S R 57 \quad 04.02 .03 .02 / S R 59$ $04.02 .04 .02 / S R 24 \quad 04.02 .04 .02 / S R 26$

\#D 5.11 .2 .5 :

$04.02 .03 .02 / S R 60 \quad 04.02 .03 .02 / S R 6104.02 .04 .02 / S R 27$

\#D 5.11 .2 .6 : 
$04.02 .03 .02 / \mathrm{SR} 63$

$04.02 .03 .02 / \mathrm{SR} 66$

$04.02 .04 .02 /$ SR31

\#D 5.12 :

$04.02 .05 .02 /$ SR1 9

\#D 5.12 .1 :

$04.02 .05 .02 /$ SR0 $704.02 .05 .02 /$ SR0 $804.02 .05 .02 / S R 11$ $04.02 .05 .02 /$ SR13 $04.02 .05 .02 /$ SR1 4

\#D 5.12 .2 :

$04.02 .05 .02 /$ SR07 $04.02 .05 .02 / S R 08 \quad 04.02 .05 .02 / S R 12$

$04.02 .05 .02 /$ SR15 $04.02 .05 .02 /$ SR16 $04.02 .05 .02 /$ SR17

$04.02 .05 .02 / S R 2504.02 .05 .02 / S R 2609.02 .03 /$ SR02

\#D $5.12 .2 .1:$

$04.02 .05 .02 /$ SR11 $04.02 .05 .02 /$ SR13 $04.02 .05 .02 /$ SR1 4

$04.02 .05 .02 /$ SR35 $04.02 .05 .02 /$ SR39 $04.02 .05 .02 /$ SR51

$09.01 .03 .01 /$ SR01 $09.01 .03 .01 /$ SR11 $09.01 .03 .02 /$ SR01

$09.01 .03 .02 /$ SR11 $09.02 .03 /$ SR01

\#D $5.12 .2 .2:$

$04.02 .05 .02 /$ SR31 $04.02 .05 .02 /$ SR35

\#D 5.12 .2 .3 :

$04.02 .05 .02 /$ SR3 $704.02 .05 .02 /$ SR38 $04.02 .05 .02 /$ SR39

$04.02 .05 .02 / S R 40 \quad 04.02 .05 .02 / S R 41$

\#D $5.12 .2 .4:$

$04.02 .05 .02 /$ SR50 $04.02 .05 .02 /$ SR51 $04.02 .05 .02 /$ SR52

$04.02 .05 .02 /$ SR53 09.01.03.01/SR12 09.01.03.02/SR12

\#D 5.13 :

$04.02 .05 .03 /$ SR18

\#D 5.13 .1 :

$04.02 .05 .03 /$ SR0 $604.02 .05 .03 /$ SR0 $704.02 .05 .03 /$ SR10 $04.02 .05 .03 /$ SR12 $04.02 .05 .03 /$ SR13

\#D 5.13 .2 :

$04.02 .05 .03 /$ SR0 $604.02 .05 .03 /$ SR0 $704.02 .05 .03 /$ SR1 1

$04.02 .05 .03 /$ SR14 $04.02 .05 .03 /$ SR15 $04.02 .05 .03 /$ SR16

$04.02 .05 .03 / S R 2404.02 .05 .03 / S R 2509.02 .03 /$ SR02

\#D 5.13.2.1:

$04.02 .05 .03 /$ SR10 $04.02 .05 .03 /$ SR12 $04.02 .05 .03 /$ SR13

$04.02 .05 .03 /$ SR30 $04.02 .05 .03 /$ SR31 $04.02 .05 .03 /$ SR3 8

$04.02 .05 .03 /$ SR39 $04.02 .05 .03 /$ SR4 $004.02 .05 .03 /$ SR 41

$04.02 .05 .03 /$ SR4 $604.02 .05 .03 /$ SR4 $704.02 .05 .03 / S R 48$

$04.02 .05 .03 /$ SR50 $04.02 .05 .03 /$ SR51 $04.02 .05 .03 /$ SR52

09.01.03.01/SR01 09.01.03.02/SR01 09.02.03/SR01

\#D 5.13 .2 .2 : 
$04.02 .05 .03 / S R 2904.02 .05 .03 / S R 30 \quad 04.02 .05 .03 / S R 31$

\#D 5.13 .2 .3 :

$04.02 .05 .03 / S R 3604.02 .05 .03 / S R 3704.02 .05 .03 / S R 38$

$04.02 .05 .03 / S R 3904.02 .05 .03 / S R 40 \quad 04.02 .05 .03 / S R 41$

\#D 5.13 .2 .4 :

$04.02 .05 .03 / S R 4404.02 .05 .03 / S R 4504.02 .05 .03 / S R 46$

$04.02 .05 .03 / S R 4704.02 .05 .03 / S R 48$

\#D 5.13 .2 .5 :

$04.02 .05 .03 / S R 4904.02 .05 .03 / S R 50 \quad 04.02 .05 .03 / S R 51$

$04.02 .05 .03 / S R 5209.01 .03 .01 / S R 1209.01 .03 .02 / S R 12$

\#D $5.14:$

$04.02 .05 .04 /$ SR10

\#D 5.14.1:

$04.02 .05 .04 / \mathrm{SR} 0104.02 .05 .04 / \mathrm{SR} 03 \quad 04.02 .05 .04 / \mathrm{SR} 04$

\#D 5.14 .2 :

$04.02 .05 .01 / S R 2104.02 .05 .04 / S R 0204.02 .05 .04 / S R 07$

$04.02 .05 .04 / S R 1604.02 .05 .04 / S R 17$

\#D 5.14 .2 .1 :

$04.02 .05 .01 / S R 20 \quad 04.02 .05 .02 / S R 40 \quad 04.02 .05 .02 / S R 41$

$04.02 .05 .04 /$ SR0 $104.02 .05 .04 / S R 0304.02 .05 .04 / S R 04$

$04.02 .05 .04 /$ SR0 $709.01 .03 .01 /$ SR11 09.01.03.02/SR11

$09.02 .03 /$ SR0 6

\#D 5.14 .2 .2 :

$04.02 .05 .01 /$ SR75 $04.02 .05 .01 / S R 76 \quad 04.02 .05 .01 / S R 77$

$04.02 .05 .01 /$ SR79 $04.02 .05 .04 / S R 0504.02 .05 .04 / S R 06$

$04.02 .05 .04 / \mathrm{SR} 1904.02 .05 .04 / \mathrm{SR} 20 \quad 04.02 .05 .04 / \mathrm{SR} 21$

$09.01 .03 .01 /$ SR15 $09.01 .03 .02 /$ SR15

\#D 5.15.1:

$04.03 .02 .01 /$ SR02 $04.03 .02 .01 /$ SR0 5

$04.03 .02 .01 /$ SR10 $04.03 .02 .02 /$ SR17

$04.03 .02 .02 / S R 2104.03 .02 .02 / S R 27$

$04.03 .02 .01 /$ SR0 9

$04.03 .02 .02 / S R 20$

\#D 5.15 .2 :

$04.03 .02 .01 / \mathrm{SR} 0104.03 .02 .01 / \mathrm{SR} 0304.03 .02 .01 / \mathrm{SR} 04$

$04.03 .02 .01 /$ SR0 $604.03 .02 .01 /$ SR0 8

\#D 5.15 .3 :

$04.02 .01 .02 / S R 4804.02 .01 .02 / S R 4904.02 .02 .02 / S R 49$ $04.02 .02 .02 /$ SR50 $04.02 .03 .02 /$ SR65 $04.02 .03 .02 /$ SR 66 $04.02 .04 .02 /$ SR30 $04.02 .04 .02 / S R 3104.02 .05 .02 / S R 52$ $04.02 .05 .02 /$ SR53 $04.02 .05 .03 /$ SR51 $04.02 .05 .03 /$ SR52 $04.02 .05 .04 / S R 2004.02 .05 .04 / S R 2104.03 .02 .02 / S R 13$ $04.03 .02 .02 /$ SR1 $404.03 .02 .02 /$ SR33

\#D 5.15 .3 .1 :

$04.02 .01 .01 / \mathrm{SR} 2104.02 .01 .01 / \mathrm{SR} 2204.02 .02 .01 / \mathrm{SR} 22$ 
$04.02 .02 .01 / \mathrm{SR} 2304.02 .03 .01 / \mathrm{SR} 48 \quad 04.02 .03 .01 / \mathrm{SR} 49$

$04.02 .04 .01 /$ SR3 $84.02 .04 .01 /$ SR3 $904.02 .05 .01 /$ SR2 8

$04.02 .05 .01 /$ SR29 $04.02 .05 .01 /$ SR5 $704.02 .05 .01 /$ SR5 8

$04.03 .02 .01 /$ SR01 $04.03 .02 .01 /$ SR02 $04.03 .02 .01 /$ SR03

$04.03 .02 .01 /$ SR0 $404.03 .02 .01 /$ SR0 $504.03 .02 .01 /$ SR0 6

$04.03 .02 .02 /$ SR30 $04.03 .02 .02 /$ SR31 $04.03 .02 .02 /$ SR3 2

$09.01 .03 .01 /$ SR12 $09.01 .03 .01 /$ SR16 $09.01 .03 .02 /$ SR12

$09.01 .03 .02 /$ SR16 $09.02 .03 /$ SR07

\#D $5.15 .3 .2:$

$04.02 .01 .01 / \mathrm{SR} 2104.02 .01 .01 / \mathrm{SR} 22 \quad 04.02 .02 .01 / \mathrm{SR} 22$

$04.02 .02 .01 / \mathrm{SR} 2304.02 .03 .01 / \mathrm{SR} 48 \quad 04.02 .03 .01 / \mathrm{SR} 49$

$04.02 .04 .01 /$ SR38 $04.02 .04 .01 /$ SR39 $04.02 .05 .01 /$ SR2 8

$04.02 .05 .01 /$ SR29 $04.02 .05 .01 /$ SR57 $04.02 .05 .01 /$ SR5 8

$04.03 .02 .01 /$ SR02 $04.03 .02 .01 /$ SR0 $404.03 .02 .01 /$ SR0 5

$04.03 .02 .01 /$ SR0 $604.03 .02 .02 / S R 21 \quad 04.03 .02 .02 / S R 26$

$04.03 .02 .02 /$ SR2 $704.03 .02 .02 /$ SR30 $04.03 .02 .02 /$ SR3 1

$04.03 .02 .02 /$ SR32 $09.01 .03 .01 /$ SR17 $09.01 .03 .02 /$ SR 17

\#D $5.16:$

$04.03 .04 .02 /$ SR01 $04.03 .04 .02 /$ SR0 $204.03 .04 .02 / S R 03$

$04.03 .04 .02 /$ SR0 $404.03 .04 .02 /$ SR10 $04.03 .04 .02 /$ SR 11

$04.03 .04 .02 /$ SR12 $04.03 .04 .02 /$ SR13

\#D $5.17:$

$04.03 .04 .02 /$ SR05 $04.03 .04 .02 /$ SR0 $04.03 .04 .02 /$ SR0 7

$04.03 .04 .02 /$ SR08 $04.03 .04 .02 /$ SR09 $04.03 .04 .02 /$ SR 10

$04.03 .04 .02 /$ SR11 $04.03 .04 .02 /$ SR12 $04.03 .04 .02 /$ SR 13

\#D 5.18 .1 .2 :

$05.02 /$ SR30 $05.02 / S R 3105.02 / S R 32$

\#D $5.18 .2 .2:$

$05.02 /$ SR30 $05.02 /$ SR $3105.02 /$ SR3 2

\#D $5.18 .3 .2:$

$05.02 /$ SR30 $05.02 /$ SR31 $05.02 /$ SR32

\#D $5.18 .4 .2:$

$05.02 /$ SR30 $05.02 /$ SR3 $05.02 /$ SR32

\#D $5.18 .5 .2:$

$05.02 /$ SR30 $05.02 /$ SR31 $05.02 /$ SR32

\#D $5.18 .6 .2:$

$05.02 /$ SR30 $05.02 / S R 3105.02 / S R 32$

\#D $6:$

$05.01 .02 /$ SR01

\#D $6.1:$

05.01.02/SR02 05.01.02/SR06 09.01.02.01/SR06 09.01.02.01/SR07 09.01.02.01/SR08 09.01.02.01/SR09 09.01.02.01/SR10

$09.01 .02 .01 /$ SR11 09.01.02.02/SR06 09.01.02.02/SR07 
09.01.02.02/SR08 09.01.02.02/SR09 09.01.02.02/SR10

$09.01 .02 .02 /$ SR11 $09.02 .02 /$ SR09

\#D 6.2 :

05.01.02/SR02 05.01.02/SR03 05.01.02/SR0 $405.01 .02 /$ SR10

09.01.02.01/SR06 09.01.02.01/SR07 09.01.02.01/SR0 8

09.01.02.01/SR09 09.01.02.01/SR10 09.01.02.01/SR11

$09.01 .02 .02 /$ SR0 $609.01 .02 .02 /$ SR0 $09.01 .02 .02 /$ SR0 8

$09.01 .02 .02 /$ SR09 $09.01 .02 .02 /$ SR10 $09.01 .02 .02 /$ SR11 $09.02 .02 /$ SR0 4

$09.02 .02 /$ SR0 $509.02 .02 /$ SR0 $609.02 .02 /$ SR0 $709.02 .02 /$ SR0 8

$09.02 .02 /$ SR0 9

\#D $6.3:$

$06.03 / S R 04$

\#D $6.4:$

06.03/SR02 06.03/SR03 09.01.04.01/SR07 09.01.04.02/SR07

\#D $6.4 .1:$

$04.01 / \mathrm{SR} 02$

\#D $6.5:$

$04.03 .03 /$ SR0 8

\#D 6.5.1:

$04.03 .03 /$ SR09

\#D $6.5 .2:$

$04.03 .03 /$ SR09

\#D 6.5.3:

$04.03 .03 /$ SR10

\#D $6.5 .4:$

$04.03 .03 /$ SR10 $04.03 .03 /$ SR11

\#D $6.5 .4 .1:$

$09.01 .03 .01 /$ SR10 $09.01 .03 .02 /$ SR10

\#D $6.6 .1:$

$06.02 .02 / S R 0406.02 .02 / S R 05$

\#D $6.6 .2:$

$06.02 .02 /$ SR0 $606.02 .02 /$ SR09 $09.02 .03 /$ SR0 8

\#D 6.6.2.1:

$06.02 .02 /$ SR0 7

\#D $6.6 .2 .2:$

$06.02 .02 /$ SR07

\#D $6.6 .2 .3:$

$06.02 .02 / \mathrm{SR} 07$ 


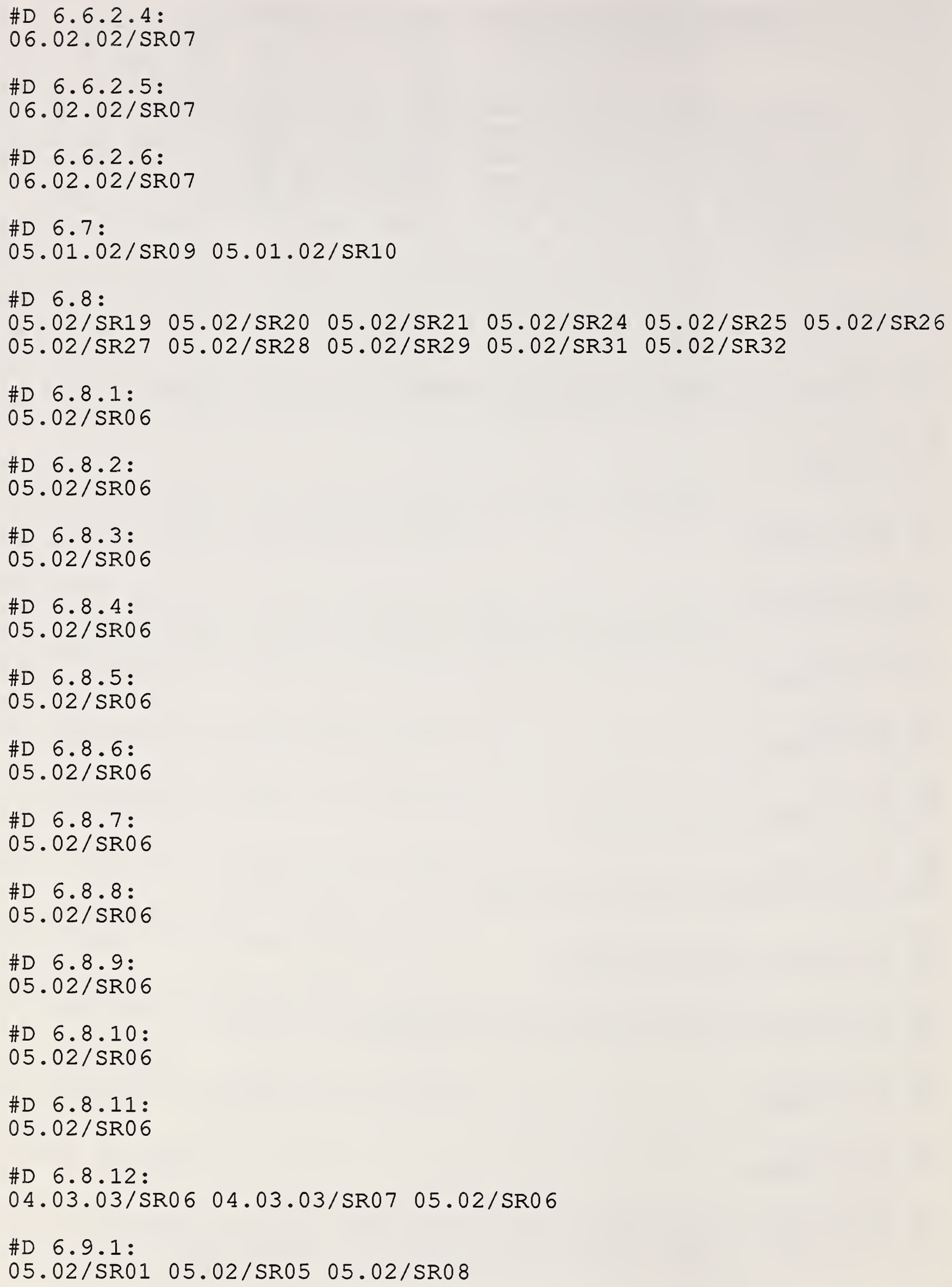


\#D 6.9.2:

05.02/SR02 05.02/SR05 05.02/SR0 8

\#D 6.10.1.1:

04.02.01.01/SR06 04.02.01.01/SR07 04.02.01.01/SR08

04.02.01.01/SR09 04.02.01.01/SR10 04.02.01.02/SR29

$04.02 .01 .02 /$ SR31 $04.02 .01 .02 /$ SR32 $04.02 .01 .02 /$ SR33

04.02.01.02/SR36 04.02.01.02/SR37 04.02.01.02/SR38

\#D 6.10.1.2:

04.02.01.01/SR06 04.02.01.01/SR07 04.02.01.01/SR08

04.02.01.01/SR09 $04.02 .01 .01 /$ SR10 $04.02 .01 .02 /$ SR26

04.02.01.02/SR29 $04.02 .01 .02 /$ SR30 $04.02 .01 .02 / S R 31$

04.02.01.02/SR32 04.02.01.02/SR33 04.02.01.02/SR34

$04.02 .01 .02 / \mathrm{SR} 3604.02 .01 .02 / \mathrm{SR} 3704.02 .01 .02 / \mathrm{SR} 38$

\#D 6.10 .1 .2 .1 :

$09.01 .03 .01 /$ SR0 $409.01 .03 .02 / S R 04$

\#D 6.10 .1 .3 :

04.02.01.01/SR15 $04.02 .01 .01 /$ SR16 $04.02 .01 .02 /$ SR39

04.02.01.02/SR41 $04.02 .01 .02 / S R 4404.02 .01 .02 / S R 45$

\#D 6.10 .1 .4 :

04.02.01.01/SR15 $04.02 .01 .01 / S R 1604.02 .01 .02 / S R 27$

$04.02 .01 .02 / S R 3904.02 .01 .02 / S R 4004.02 .01 .02 / S R 41$

$04.02 .01 .02 /$ SR $4404.02 .01 .02 / S R 45$

\#D 6.10 .1 .5 :

$04.02 .01 .01 /$ SR15 $04.02 .01 .01 /$ SR16 $04.02 .01 .02 / S R 27$

$04.02 .01 .02 / S R 3904.02 .01 .02 / S R 4004.02 .01 .02 / S R 41$

$04.02 .01 .02 / S R 4404.02 .01 .02 / S R 45$

\#D 6.10 .1 .6 :

$04.02 .01 .01 /$ SR15 $04.02 .01 .01 / S R 1604.02 .01 .02 / S R 27$

$04.02 .01 .02 / S R 3904.02 .01 .02 / S R 4004.02 .01 .02 / S R 41$

$04.02 .01 .02 / S R 4404.02 .01 .02 / S R 45$

\#D 6.10 .1 .7 :

$04.02 .01 .02 / S R 1904.02 .01 .02 / S R 21$

\#D 6.10 .2 :

$04.02 .01 .02 / S R 20 \quad 04.02 .01 .02 / S R 2104.02 .01 .02 / S R 22$

$04.02 .01 .02 /$ SR23 $04.02 .01 .02 /$ SR24 $04.02 .01 .02 /$ SR25 $09.02 .03 /$ SR01

$09.02 .03 / \mathrm{SR} 03$

\#D 6.10 .2 .1 :

$04.02 .01 .02 / \mathrm{SR} 26$

\#D 6.10 .2 .2 :

$04.02 .01 .02 / S R 27$

\#D 6.10.2.3: 
$04.02 .01 .02 /$ SR2 8

\#D $6.11 .1 .1:$

$04.02 .02 .01 /$ SR0 $604.02 .02 .01 /$ SR07 $04.02 .02 .01 /$ SR0 8

$04.02 .02 .01 /$ SR0 $904.02 .02 .01 /$ SR10 $04.02 .02 .02 /$ SR2 9

$04.02 .02 .02 /$ SR31 $04.02 .02 .02 /$ SR32 $04.02 .02 .02 /$ SR33

$04.02 .02 .02 /$ SR $3604.02 .02 .02 /$ SR37 $04.02 .02 .02 /$ SR3 8

\#D $6.11 .1 .2:$

$04.02 .02 .01 /$ SR0 $604.02 .02 .01 /$ SR0 $704.02 .02 .01 / S R 08$

$04.02 .02 .01 /$ SR0 $904.02 .02 .01 /$ SR10 $04.02 .02 .02 /$ SR2 6

$04.02 .02 .02 /$ SR2 $904.02 .02 .02 /$ SR30 $04.02 .02 .02 /$ SR3 1

$04.02 .02 .02 /$ SR32 $04.02 .02 .02 /$ SR33 $04.02 .02 .02 /$ SR3 4

$04.02 .02 .02 / S R 3604.02 .02 .02 /$ SR $3704.02 .02 .02 /$ SR 38

\#D 6.11.1.2.1:

$09.01 .03 .01 /$ SR05 $09.01 .03 .02 /$ SR05

\#D $6.11 .1 .3:$

$04.02 .02 .01 /$ SR15 $04.02 .02 .01 /$ SR16 $04.02 .02 .02 / S R 39$

$04.02 .02 .02 / S R 4104.02 .02 .02 / S R 44 \quad 04.02 .02 .02 / S R 45$

\#D $6.11 .1 .4:$

$04.02 .02 .01 /$ SR15 $04.02 .02 .01 /$ SR16 $04.02 .02 .01 /$ SR17

$04.02 .02 .02 / S R 2704.02 .02 .02 / S R 3904.02 .02 .02 / S R 40$

$04.02 .02 .02 /$ SR $4104.02 .02 .02 / S R 44 \quad 04.02 .02 .02 / S R 45$

$04.02 .02 .02 /$ SR 46

\#D $6.11 .1 .5:$

$04.02 .02 .01 /$ SR15 $04.02 .02 .01 /$ SR1 $604.02 .02 .01 /$ SR1 7

$04.02 .02 .02 /$ SR2 $704.02 .02 .02 /$ SR3 $94.02 .02 .02 / S R 40$

$04.02 .02 .02 / S R 4104.02 .02 .02 / S R 44 \quad 04.02 .02 .02 / S R 45$

$04.02 .02 .02 /$ SR 46

\#D $6.11 .1 .6:$

$04.02 .02 .01 /$ SR15 $04.02 .02 .01 /$ SR16 $04.02 .02 .01 /$ SR1 7

$04.02 .02 .02 /$ SR2 $704.02 .02 .02 /$ SR $3904.02 .02 .02 / S R 40$

$04.02 .02 .02 /$ SR $4104.02 .02 .02 /$ SR $44 \quad 04.02 .02 .02 / S R 45$

$04.02 .02 .02 /$ SR 46

\#D $6.11 .1 .7:$

$04.02 .02 .02 /$ SR19 $04.02 .02 .02 /$ SR21

\#D $6.11 .2:$

$04.02 .02 .02 / S R 20 \quad 04.02 .02 .02 / S R 21 \quad 04.02 .02 .02 / S R 22$

$04.02 .02 .02 /$ SR23 $04.02 .02 .02 /$ SR2 $404.02 .02 .02 /$ SR25 $09.02 .03 /$ SR01

$09.02 .03 / \mathrm{SR} 03$

\#D $6.11 .2 .1:$

$04.02 .02 .02 /$ SR2 6

\#D $6.11 .2 .2:$

$04.02 .02 .02 /$ SR27 
\#D $6.11 .2 .3:$

$04.02 .02 .02 / \mathrm{SR} 28$

\#D $6.12 .1 .1:$

$04.02 .03 .01 / \mathrm{SR} 1904.02 .03 .01 / \mathrm{SR} 2104.02 .03 .01 / \mathrm{SR} 22$

$04.02 .03 .01 /$ SR28 $04.02 .03 .02 / S R 2604.02 .03 .02 / S R 40$

$04.02 .03 .02 /$ SR $4704.02 .03 .03 /$ SR01 $04.02 .04 .01 /$ SR12

$04.02 .04 .01 /$ SR14 $04.02 .04 .01 /$ SR15 $04.02 .04 .01 /$ SR20

$04.02 .04 .02 /$ SR13 $04.02 .04 .02 /$ SR2 0

\#D $6.12 .1 .2:$

$04.02 .03 .01 /$ SR19 $04.02 .03 .01 /$ SR21 $04.02 .03 .01 / S R 22$

$04.02 .03 .01 / \mathrm{SR} 28 \quad 04.02 .03 .02 / \mathrm{SR} 26 \quad 04.02 .03 .02 / \mathrm{SR} 30$

$04.02 .03 .02 /$ SR32 $04.02 .03 .02 /$ SR33 $04.02 .03 .02 /$ SR35

$04.02 .03 .02 /$ SR4 $004.02 .03 .02 /$ SR $4704.02 .03 .03 /$ SR0 1

$04.02 .04 .01 /$ SR12 $04.02 .04 .01 /$ SR14 $04.02 .04 .01 /$ SR15

$04.02 .04 .01 /$ SR20 $04.02 .04 .02 /$ SR13 $04.02 .04 .02 /$ SR2 0

\#D $6.12 .1 .2 .1:$

$04.02 .03 .02 /$ SR31 $04.02 .03 .02 /$ SR3 $404.02 .03 .02 /$ SR3 6

$04.02 .03 .02 /$ SR3 $04.02 .03 .03 /$ SR0 $904.02 .03 .03 /$ SR15

$04.02 .03 .03 /$ SR1 $604.02 .04 .02 /$ SR10 $09.01 .03 .01 /$ SR0 6

$09.01 .03 .02 /$ SR0 $609.02 .03 /$ SR0 4

\#D $6.12 \cdot 1 \cdot 3:$

$04.02 .03 .01 /$ SR29 $04.02 .03 .01 /$ SR30 $04.02 .03 .01 /$ SR61

\#D $6.12 .1 .4:$

$04.02 .03 .01 /$ SR2 $904.02 .03 .01 /$ SR30 $04.02 .03 .01 /$ SR61

\#D $6.12 \cdot 1.5:$

$04.02 .03 .01 /$ SR2 $904.02 .03 .01 /$ SR30 $04.02 .03 .01 /$ SR 61

\#D 6.12.1.6:

$04.02 .03 .01 /$ SR29 $04.02 .03 .01 /$ SR30 $04.02 .03 .01 /$ SR3 7

$04.02 .03 .01 /$ SR53 $04.02 .03 .01 /$ SR55 $04.02 .03 .01 /$ SR5 7

$04.02 .03 .02 /$ SR2 $704.02 .03 .02 / S R 48 \quad 04.02 .03 .02 / S R 49$

$04.02 .03 .02 /$ SR51 $04.02 .03 .02 /$ SR53 $04.02 .03 .02 /$ SR55

$04.02 .03 .02 /$ SR5 $904.02 .04 .01 /$ SR21 $04.02 .04 .01 /$ SR2 9

$04.02 .04 .02 /$ SR2 $104.02 .04 .02 /$ SR2 $204.02 .04 .02 / S R 26$

\#D $6.12 .1 .7:$

$04.02 .03 .01 /$ SR29 $04.02 .03 .01 /$ SR30 $04.02 .03 .01 /$ SR3 7

$04.02 .03 .01 /$ SR53 $04.02 .03 .01 /$ SR5 $404.02 .03 .01 /$ SR5 6

$04.02 .03 .02 /$ SR2 $704.02 .03 .02 / S R 48 \quad 04.02 .03 .02 /$ SR 49

$04.02 .03 .02 /$ SR51 $04.02 .03 .02 /$ SR52 $04.02 .03 .02 /$ SR5 4

$04.02 .03 .02 /$ SR59 $04.02 .04 .01 /$ SR21 $04.02 .04 .01 /$ SR 29

$04.02 .04 .02 /$ SR21 $04.02 .04 .02 /$ SR2 $204.02 .04 .02 / S R 26$

\#D $6.12 .1 .8:$

$04.02 .03 .01 /$ SR29 $04.02 .03 .01 /$ SR30 $04.02 .03 .01 /$ SR3 7

$04.02 .03 .01 /$ SR53 $04.02 .03 .01 /$ SR5 $404.02 .03 .01 /$ SR5 6

$04.02 .03 .02 /$ SR2 $704.02 .03 .02 /$ SR $4804.02 .03 .02 /$ SR 49

$04.02 .03 .02 /$ SR51 $04.02 .03 .02 /$ SR52 $04.02 .03 .02 /$ SR5 4 
$04.02 .03 .02 / S R 5904.02 .04 .01 / S R 2104.02 .04 .01 / S R 29$

$04.02 .04 .02 / S R 2104.02 .04 .02 / S R 2204.02 .04 .02 / S R 26$

\#D $6.12 .1 .9:$

$04.02 .03 .02 /$ SR19 $04.02 .03 .02 /$ SR21

\#D 6.12 .2 :

$04.02 .03 .02 / S R 20 \quad 04.02 .03 .02 / S R 2104.02 .03 .02 / S R 22$

$04.02 .03 .02 /$ SR23 $04.02 .03 .02 /$ SR2 $404.02 .03 .02 /$ SR2 5

$04.02 .04 .02 /$ SR0 $84.02 .04 .02 /$ SR09 $09.02 .03 /$ SR01 $09.02 .03 /$ SR03

\#D $6.12 .2 .1:$

$04.02 .03 .02 /$ SR2 6

\#D $6.12 .2 .2:$

$04.02 .03 .02 / S R 26 \quad 04.02 .03 .02 / S R 27 \quad 04.02 .03 .02 / S R 28$

$04.02 .03 .02 /$ SR3 $404.02 .04 .02 /$ SR10

\#D $6.12 .2 .3:$

$04.02 .03 .02 /$ SR2 7

\#D $6.12 .2 .4:$

$04.02 .03 .02 /$ SR28

\# $6.12 .2 .5:$

$04.02 .03 .02 /$ SR29

\#D $6.13 .1 .1:$

$04.02 .04 .01 / S R 2104.02 .04 .01 / S R 43 \quad 04.02 .04 .01 / S R 45$

$04.02 .04 .01 /$ SR $4704.02 .04 .01 /$ SR52

\#D 6.13.1.2:

$04.02 .04 .01 / S R 2104.02 .04 .01 / S R 43 \quad 04.02 .04 .01 / S R 44$ $04.02 .04 .01 /$ SR $4604.02 .04 .01 /$ SR52

\#D $6.13 .1 .3:$

$04.02 .04 .01 / S R 2104.02 .04 .01 / S R 43 \quad 04.02 .04 .01 / S R 44$

$04.02 .04 .01 / S R 4604.02 .04 .01 /$ SR52

\#D $6.13 .1 .4:$

$04.02 .04 .01 / S R 77 \quad 04.02 .04 .01 / S R 78 \quad 04.02 .04 .01 / S R 79$

$04.02 .04 .01 /$ SR81 $04.02 .04 .01 /$ SR82

\#D $6.13 .1 .5:$

$04.02 .04 .01 / S R 7704.02 .04 .01 / S R 78 \quad 04.02 .04 .01 / S R 79$

$04.02 .04 .01 /$ SR81 $04.02 .04 .01 /$ SR82

\#D 6.14.1.1:

$04.02 .05 .01 /$ SR0 $64.02 .05 .01 /$ SR0 $804.02 .05 .01 /$ SR0 9 $04.02 .05 .02 /$ SR3 6

\#D 6.14 .1 .2 :

$04.02 .05 .01 /$ SR0 $604.02 .05 .01 /$ SR0 $804.02 .05 .01 /$ SR0 9

$04.02 .05 .02 /$ SR27 $04.02 .05 .02 /$ SR31 $04.02 .05 .02 /$ SR 32 
$04.02 .05 .02 / \mathrm{SR} 3404.02 .05 .02 / \mathrm{SR} 36 \quad 04.02 .05 .02 / \mathrm{SR} 42$ $04.02 .05 .02 / S R 4304.02 .05 .04 /$ SR0 $904.02 .05 .04 / S R 14$

\#D $6.14 .1 .2 .1:$

$04.02 .05 .02 /$ SR33 09.01.03.01/SR0 $809.01 .03 .02 /$ SR0 $809.02 .03 / S R 05$

\# $6.14 .1 .3:$

$04.02 .05 .01 / \mathrm{SR} 20 \quad 04.02 .05 .01 / \mathrm{SR} 2104.02 .05 .01 / \mathrm{SR} 22$

$04.02 .05 .01 / S R 2304.02 .05 .02 / S R 42 \quad 04.02 .05 .02 / S R 43$

$04.02 .05 .02 / \mathrm{SR} 4404.02 .05 .02 / \mathrm{SR} 45 \quad 04.02 .05 .02 / \mathrm{SR} 46$

\#D $6.14 .1 .4:$

$04.02 .05 .01 / \mathrm{SR} 20 \quad 04.02 .05 .01 / \mathrm{SR} 2104.02 .05 .01 / \mathrm{SR} 22$

$04.02 .05 .01 / \mathrm{SR} 2304.02 .05 .02 / \mathrm{SR} 2904.02 .05 .02 / \mathrm{SR} 40$

$04.02 .05 .02 / S R 4104.02 .05 .02 / S R 44 \quad 04.02 .05 .02 / S R 45$

$04.02 .05 .02 /$ SR $4604.02 .05 .02 / S R 4704.02 .05 .02 / S R 48$

$04.02 .05 .02 /$ SR 49

\#D $6.14 .1 .5:$

$04.02 .05 .02 / \mathrm{SR} 20 \quad 04.02 .05 .02 / \mathrm{SR} 22$

\#D $6.14 .2:$

$04.02 .05 .02 / \mathrm{SR} 2104.02 .05 .02 / \mathrm{SR} 22 \quad 04.02 .05 .02 / \mathrm{SR} 23$

$04.02 .05 .02 /$ SR2 $404.02 .05 .02 /$ SR25 $04.02 .05 .02 / S R 2609.02 .03 / S R 01$

$09.02 .03 / \mathrm{SR} 03$

\# $6.14 .2 .1:$

$04.02 .05 .02 / \mathrm{SR} 27 \quad 04.02 .05 .02 / \mathrm{SR} 28 \quad 04.02 .05 .02 / \mathrm{SR} 29$

$04.02 .05 .02 / S R 31$

\#D $6.14 .2 .2:$

$04.02 .05 .02 /$ SR2 $804.02 .05 .02 / S R 29$

\#D $6.14 .2 .3:$

$04.02 .05 .02 / \mathrm{SR} 30$

\#D 6.15.1.1:

$04.02 .05 .01 / \mathrm{SR} 4204.02 .05 .01 / \mathrm{SR} 4304.02 .05 .01 / \mathrm{SR} 44$

$04.02 .05 .01 /$ SR $4504.02 .05 .01 /$ SR $4604.02 .05 .03 /$ SR 32

$04.02 .05 .03 /$ SR3 $404.02 .05 .03 /$ SR35 $04.02 .05 .03 /$ SR3 6

$04.02 .05 .03 / S R 3904.02 .05 .03 / S R 40 \quad 04.02 .05 .03 / S R 41$

\# $6.15 .1 .2:$

$04.02 .05 .01 / S R 4204.02 .05 .01 / S R 4304.02 .05 .01 / S R 44$

$04.02 .05 .01 / S R 4504.02 .05 .01 / S R 4604.02 .05 .03 / S R 26$

$04.02 .05 .03 /$ SR32 $04.02 .05 .03 /$ SR33 $04.02 .05 .03 /$ SR3 4

$04.02 .05 .03 /$ SR35 $04.02 .05 .03 /$ SR36 $04.02 .05 .03 /$ SR3 7

$04.02 .05 .03 /$ SR39 $04.02 .05 .03 /$ SR40 $04.02 .05 .03 /$ SR4 1

\#D $6.15 .1 .2 .1:$

$09.01 .03 .01 /$ SR07 $09.01 .03 .02 /$ SR0 7

\# $6.15 .1 .3:$

$04.02 .05 .01 /$ SR51 $04.02 .05 .01 /$ SR52 $04.02 .05 .03 / S R 42$ 
$04.02 .05 .03 / S R 4404.02 .05 .03 / S R 47 \quad 04.02 .05 .03 / S R 48$

\#D $6.15 .1 .4:$

$04.02 .05 .01 /$ SR51 $04.02 .05 .01 /$ SR52 $04.02 .05 .03 /$ SR27

$04.02 .05 .03 / S R 4204.02 .05 .03 / S R 4304.02 .05 .03 / S R 44$

$04.02 .05 .03 / S R 4704.02 .05 .03 / S R 48$

\#D $6.15 .1 .5:$

$04.02 .05 .01 /$ SR51 $04.02 .05 .01 /$ SR52 $04.02 .05 .03 /$ SR27

$04.02 .05 .03 / S R 4204.02 .05 .03 / S R 4304.02 .05 .03 / S R 44$

$04.02 .05 .03 /$ SR $4704.02 .05 .03 / S R 48$

\#D 6.15.1.6:

$04.02 .05 .01 /$ SR51 $04.02 .05 .01 /$ SR52 $04.02 .05 .03 /$ SR27

$04.02 .05 .03 / S R 4204.02 .05 .03 / S R 4304.02 .05 .03 / S R 44$

$04.02 .05 .03 / S R 4704.02 .05 .03 / S R 48$

\#D 6.15.1.7:

$04.02 .05 .03 /$ SR19 $04.02 .05 .03 /$ SR21

\#D 6.15.2:

$04.02 .05 .03 / S R 2004.02 .05 .03 / S R 2104.02 .05 .03 / S R 22$

$04.02 .05 .03 /$ SR23 $04.02 .05 .03 /$ SR2 $404.02 .05 .03 /$ SR2 5 $09.02 .03 /$ SR0 3

\#D 6.15 .2 .1 :

$04.02 .05 .03 /$ SR2 9

\#D 6.15 .2 .2 :

$04.02 .05 .03 /$ SR 26

\#D $6.15 \cdot 2.3:$

$04.02 .05 .03 /$ SR27

\#D 6.15 .2 .4 :

$04.02 .05 .03 / \mathrm{SR} 28$

\#D 6.16.1.1:

$04.02 .05 .02 / S R 28 \quad 04.02 .05 .02 / S R 34 \quad 04.02 .05 .04 / S R 11$

$04.02 .05 .04 /$ SR13 $04.02 .05 .04 /$ SR1. 4

\#D 6.16.2:

$04.02 .05 .04 /$ SR12 $04.02 .05 .04 /$ SR13 $04.02 .05 .04 /$ SR15

$04.02 .05 .04 / S R 1604.02 .05 .04 / S R 17 \quad 09.02 .03 /$ SR0 6

\#D 6.16 .2 .1 :

$04.02 .05 .04 /$ SR1 8

\#D $6.17 .1 .1:$

$04.03 .02 .02 /$ SR 16

\#D 6.17 .1 .2 :

$04.03 .02 .02 /$ SR1 $604.03 .02 .02 /$ SR17 $04.03 .02 .02 /$ SR1 8

$04.03 .02 .02 /$ SR1 $904.03 .02 .02 /$ SR22 $04.03 .02 .02 /$ SR23 
$04.03 .02 .02 / S R 24 \quad 04.03 .02 .02 / S R 25 \quad 04.03 .02 .02 / S R 28$ $04.03 .02 .02 / S R 29$

\#D $6.17 .1 .2 .1:$

$09.01 .03 .01 /$ SR09 09.01.03.02/SR09

\#D $6.17 .1 .3:$

$04.03 .02 .02 /$ SR09 $04.03 .02 .02 /$ SR15 $04.03 .02 .02 /$ SR1 6 $04.03 .02 .02 /$ SR18 $04.03 .02 .02 /$ SR20

\#D $6.17 .2 .1:$

$04.03 .02 .02 /$ SR0 $04.03 .02 .02 /$ SR15 $04.03 .02 .02 /$ SR 33

\#D $6.17 .2 .2:$

$04.03 .02 .02 /$ SR01 $04.03 .02 .02 /$ SR02 $04.03 .02 .02 / S R 03$ $04.03 .02 .02 /$ SR15 $04.03 .02 .02 /$ SR33

\#D $6.17 .2 .3:$

$04.03 .02 .02 /$ SR0 $404.03 .02 .02 /$ SR0 $5 \quad 04.03 .02 .02 /$ SR0 6 $04.03 .02 .02 /$ SR3 3

\#D $6.17 .2 .4:$

$04.02 .01 .02 / S R 28 \quad 04.02 .02 .02 / S R 28 \quad 04.02 .03 .02 / S R 29$ $04.02 .05 .02 /$ SR30 $04.02 .05 .03 /$ SR2 $804.02 .05 .04 / S R 18$ $04.03 .02 .02 /$ SR0 8

\#D $6.17 .3:$

$04.03 .02 .02 /$ SR10 $04.03 .02 .02 /$ SRI1 $04.03 .02 .02 / S R 13$ $04.03 .02 .02 /$ SR14 $09.02 .03 /$ SR0 7

\#D $6.17 .3 .1:$

$04.03 .02 .02 /$ SR09 $04.03 .02 .02 /$ SR12 $04.03 .02 .02 /$ SR 15

\#D $6.18 .1:$

$04.01 .08 /$ SRO 4

\#D $6.18 .2:$

$04.01 .08 /$ SR0 4

\#D 6.18 .2 .3 :

$04.01 .08 /$ SR0 5

\#D 6.18 .3 :

$04.01 .08 /$ SR09

\#D $6.18 .4:$

$04.01 .08 /$ SR09

\#D 6.18 .4 .3 :

$04.01 .08 /$ SR10

\#D 6.19.1:

$02.02 .02 /$ SR 13 
\#D $6.19 .2:$

$02.02 .02 /$ SR11 $02.02 .02 /$ SR13

\#D $6.20:$

$05.03 /$ SR1 $605.03 /$ SR17 $05.03 /$ SR18 $05.03 /$ SR1 9

\#D $6.21 .1:$

$04.02 .01 .02 / S R 1704.02 .01 .02 / S R 1809.01 .03 .01 / S R 03$ $09.01 .03 .02 /$ SR03

\#D $6.21 .2:$

$04.02 .02 .02 /$ SR17 $04.02 .02 .02 /$ SR18 $09.01 .03 .01 /$ SR03 $09.01 .03 .02 /$ SR03

\#D $6.21 .3:$

$04.02 .03 .02 /$ SR17 $04.02 .03 .02 /$ SR18 $09.01 .03 .01 /$ SR0 3 $09.01 .03 .02 /$ SR03

\#D $6.21 .4:$

$04.02 .05 .02 /$ SR18 $04.02 .05 .02 /$ SR19 $09.01 .03 .01 /$ SR03 $09.01 .03 .02 /$ SR03

\#D $6.21 .5:$

$04.02 .05 .03 /$ SR17 $04.02 .05 .03 /$ SR18 $09.01 .03 .01 /$ SR03 $09.01 .03 .02 /$ SR03

\#D $6.21 .6:$

$04.02 .05 .04 /$ SR0 $804.02 .05 .04 /$ SR09 $04.02 .05 .04 /$ SR10

$09.01 .03 .01 /$ SR03 $09.01 .03 .02 /$ SR03

\#D $6.21 .7:$

$04.03 .02 .01 /$ SR0 $704.03 .02 .01 /$ SR0 $809.01 .03 .01 /$ SR03 $09.01 .03 .02 / \mathrm{SR} 03$

\#D $6.21 .8:$

06.02.02/SR12 06.02.02/SR13 09.01.04.01/SR01 09.01.04.02/SR01

\#D $6.22:$

05.02/SR1 $905.02 / S R 2005.02 / S R 21 \quad 05.02 / S R 2405.02 / S R 2505.02 / S R 26$

$05.02 / S R 2705.02 / S R 28 \quad 05.02 / S R 29 \quad 05.02 /$ SR3 $105.02 /$ SR32

\#D $6.22 .1:$

$05.02 /$ SR0 7

\#D 6.22 .2 :

$05.02 / S R 07$

\#D $6.22 .3:$

$05.02 /$ SR0 7

\#D $6.22 .4:$

$05.02 /$ SR0 7

\#D $6.22 .5:$ 
$05.02 / S R 07$

\#D $7:$

02.01.01/SR08 02.01.02.02/SR01 02.01.02.02/SR02 02.01.02.02/SR03

$02.01 .02 .02 / \mathrm{SR} 0402.01 .02 .02 / \mathrm{SR} 05 \quad 02.01 .02 .02 / \mathrm{SR} 06$

$02.01 .02 .02 /$ SR07 $02.01 .02 .02 /$ SR0 $82.01 .02 .02 /$ SR0 9

$02.01 .03 .01 /$ SR01 $02.01 .03 .01 /$ SR02 $02.01 .03 .01 /$ SR03

$02.01 .03 .03 /$ SR01 $02.01 .03 .03 /$ SR02 $02.01 .03 .03 /$ SR03 $02.03 .03 /$ SR02

$02.03 .03 /$ SR03 $02.03 .03 /$ SR0 $402.03 .03 /$ SR0 $502.03 .03 /$ SR0 6

\#D 7.1 :

02.01/SR02 02.01.01/SR01 02.01.01/SR02 02.01.01/SR03

$02.01 .01 /$ SR0 $402.01 .01 /$ SR05 $02.01 .01 /$ SR0 $602.01 .01 /$ SR0 7

$02.01 .01 /$ SR09 $02.01 .01 /$ SR10 $02.01 .02 .01 /$ SR01 $02.01 .02 .01 / S R 03$

$02.01 .02 .01 /$ SR05 $02.01 .02 .03 /$ SR01 $02.01 .02 .03 /$ SR0 2

$02.01 .03 .01 /$ SR01 $02.01 .03 .01 /$ SR02 $02.01 .03 .01 /$ SR03

$02.01 .03 .02 /$ SR01 $02.01 .03 .02 /$ SR02 $02.01 .03 .02 /$ SR0 3

$02.01 .03 .02 /$ SR0 $402.01 .03 .03 /$ SR01 $02.01 .03 .03 /$ SR0 2

$02.01 .03 .03 /$ SR0 $32.01 .03 .03 /$ SR0 $02.01 .03 .03 /$ SR0 $802.02 .01 / S R 07$

$02.02 .04 /$ SR0 $82.02 .04 /$ SR09 $02.02 .04 /$ SR10 $02.02 .04 /$ SR1 1

$02.02 .05 /$ SR01 $02.02 .05 /$ SR02 $02.02 .05 /$ SR0 $402.03 .01 /$ SR0 4

$02.03 .01 /$ SR05 $02.03 .01 /$ SR0 $602.03 .02 /$ SR01 $02.03 .02 /$ SR0 2

$02.03 .02 /$ SR0 $702.03 .03 /$ SR0 $702.03 .03 /$ SR0 $803.03 /$ SR0 $203.03 /$ SR03

$03.03 /$ SR0 $403.03 /$ SR0 $503.03 /$ SR0 $603.03 /$ SR0 $0303.03 /$ SR0 $03.03 / S R 12$

$03.03 /$ SR13 $03.03 /$ SR14 $03.03 /$ SR15 $03.03 /$ SR16 $03.03 /$ SR17 $03.03 /$ SR 18

$03.03 /$ SR19 03.04/SR02 03.04/SR03 03.04/SR04 09.01.05.01/SR03

$09.01 .05 .01 / \mathrm{SR} 0409.01 .05 .01 / \mathrm{SR} 0609.01 .05 .02 / \mathrm{SR} 03$

$09.01 .05 .02 /$ SR0 $409.01 .05 .02 /$ SR0 6

\#D $7.2:$

$02.01 .03 .01 /$ SR01 $02.01 .03 .01 /$ SR03 $02.01 .03 .02 /$ SR0 5

$02.01 .03 .02 /$ SR0 $602.01 .03 .03 /$ SR0 $102.01 .03 .03 /$ SR03 $05.03 /$ SR0 7

$05.03 /$ SR10 $05.03 /$ SR11 $05.03 /$ SR13 $09.01 .05 .01 /$ SR0 6

$09.01 .05 .02 /$ SR0 6

\#D 7.2.1:

05.03/SR01 05.03/SR05 05.03/SR08 05.03/SR12

\#D $7.3:$

$02.01 .02 .01 /$ SR0 $62.01 .02 .01 /$ SR0 $02.01 .02 .02 / S R 11$

$02.01 .02 .02 /$ SR12 $02.02 .01 /$ SR0 $02.02 .03 /$ SR02 $02.02 .03 /$ SR03

$02.02 .03 /$ SR0 $62.02 .04 /$ SR01 $02.02 .04 /$ SR02 $02.02 .04 /$ SR0 3

$02.02 .04 / S R 0402.02 .04 /$ SR0 $502.02 .04 /$ SR0 $702.02 .04 /$ SR0 8

$02.02 .04 /$ SR11 $02.02 .05 /$ SR01 $02.02 .05 /$ SR02 $02.02 .05 /$ SR0 3

$02.02 .05 /$ SR0 $402.03 .01 /$ SR01 $02.03 .01 /$ SR02 $02.03 .01 /$ SR03

$02.03 .01 /$ SR0 $402.03 .01 /$ SR0 $502.03 .01 /$ SR0 $602.03 .02 /$ SR01

$02.03 .02 /$ SR02 $02.03 .02 /$ SR03 $02.03 .02 /$ SR0 $402.03 .02 /$ SR0 5

$02.03 .02 /$ SR0 $62.03 .02 /$ SR0 $702.03 .02 /$ SR0 $0202.03 .02 /$ SR0 9

$02.03 .03 /$ SR07 09.01.05.01/SR03 09.01.05.01/SR04 09.01.05.02/SR03

$09.01 .05 .02 / S R 0409.02 .05 / S R 02$

\#D $7.3 .1:$

$02.03 .03 /$ SR10 $06.01 .02 /$ SR17 $06.01 .02 /$ SR57 $06.01 .02 /$ SR5 8 
\#D $7.3 .1 .1:$

$02.03 .03 /$ SR12 $04.01 .01 / S R 0104.01 .01 / S R 0204.01 .01 / S R 03$

$04.01 .01 /$ SR0 $704.02 .01 .01 /$ SR03 $04.02 .01 .02 /$ SR03 $04.03 .01 /$ SR0 7 $04.03 .04 .01 /$ SR 15

\#D $7.3 .1 .2:$

$02.03 .03 /$ SR12 $04.01 .01 /$ SR04 $04.01 .01 / S R 0504.01 .01 / S R 06$

$04.01 .01 /$ SR0 $04.02 .01 .01 /$ SR03 $04.02 .01 .02 /$ SR03 $04.03 .01 /$ SR0 7

\#D $7.3 \cdot 1.3:$

$02.03 .03 /$ SR11 $04.01 .02 /$ SR01 $04.01 .02 /$ SR02 $04.01 .02 /$ SR03

$04.01 .02 /$ SR0 $704.02 .02 .01 /$ SR03 $04.02 .02 .02 /$ SR03 $04.03 .01 /$ SR0 7

$06.01 .02 / \operatorname{SR} 59$

\#D $7.3 .1 .4:$

$02.03 .03 /$ SR11 $04.01 .02 /$ SR0 $404.01 .02 /$ SR0 $504.01 .02 /$ SR0 6

$04.01 .02 /$ SR0 $704.02 .02 .01 /$ SR03 $04.02 .02 .02 /$ SR03 $04.03 .01 / S R 07$

$06.01 .02 /$ SR 59

\#D $7.3 \cdot 1 \cdot 5:$

$02.03 .03 /$ SR15 $04.01 .03 /$ SR01 $04.01 .03 /$ SR02 $04.01 .03 /$ SR03

$04.01 .03 /$ SR09 $04.01 .03 /$ SR11 $04.02 .03 .01 /$ SR01 $04.02 .03 .01 / S R 02$

$04.02 .03 .01 /$ SR11 $04.02 .03 .02 /$ SR03 $04.03 .01 /$ SR0 $06.01 .02 /$ SR 60

\#D $7.3 .1 \cdot 6:$

$02.03 .03 /$ SR15 $04.01 .03 /$ SR04 $04.01 .03 /$ SR05 $04.01 .03 /$ SR0 6

$04.01 .03 /$ SR0 $904.01 .03 /$ SR10 $04.02 .03 .01 /$ SR01 $04.02 .03 .01 /$ SR02

$04.02 .03 .01 /$ SR11 $04.02 .03 .02 /$ SR03 $04.03 .01 /$ SR0 $06.01 .02 /$ SR 60

\#D $7.3 .1 .7:$

$02.03 .03 /$ SR1 $604.01 .04 /$ SR01 $04.01 .04 /$ SR02 $04.01 .04 /$ SR03

$04.01 .04 /$ SR07 04.01.04/SR09 04.02.04.01/SR01 04.02.04.01/SR02 $04.02 .04 .01 /$ SR05 $04.02 .04 .02 /$ SR01 $04.03 .01 /$ SR0 $706.01 .02 /$ SR61 $06.01 .02 /$ SR 62

\#D $7.3 .1 .8:$

$02.03 .03 /$ SR1 $604.01 .04 /$ SR0 $404.01 .04 /$ SR05 $04.01 .04 /$ SR0 6

$04.01 .04 /$ SR07 04.01.04/SR08 $04.01 .04 /$ SR09 $04.02 .04 .01 /$ SR01

$04.02 .04 .01 / \mathrm{SR} 0204.02 .04 .01 / \mathrm{SR} 05 \quad 04.02 .04 .02 / \mathrm{SR} 0104.03 .01 / \mathrm{SR} 07$

$06.01 .02 /$ SR61 $06.01 .02 /$ SR 62

\#D $7.3 \cdot 1 \cdot 9:$

$02.03 .03 /$ SR13 $04.01 .05 /$ SR01 $04.01 .05 /$ SR02 $04.01 .05 /$ SR03

$04.01 .05 /$ SR0 $704.01 .05 /$ SR08 $04.02 .05 .01 /$ SR03 $04.02 .05 .02 /$ SR03

$04.03 .01 /$ SR07 $06.01 .02 /$ SR 63

\#D $7.3 .1 .10:$

$02.03 .03 /$ SR13 $04.01 .05 /$ SR0 $404.01 .05 /$ SR0 $504.01 .05 /$ SR0 6

$04.01 .05 /$ SR0 $04.01 .05 /$ SR0 $804.02 .05 .01 /$ SR03 $04.02 .05 .02 /$ SR03

$04.03 .01 /$ SR0 $706.01 .02 /$ SR 63

\#D $7.3 .1 .11:$

$02.03 .03 /$ SR14 $04.01 .06 /$ SR01 $04.01 .06 /$ SR02 $04.01 .06 /$ SR03

$04.01 .06 /$ SR07 $04.01 .06 /$ SR08 $04.02 .05 .01 /$ SR0 $404.02 .05 .02 /$ SR0 4 
$04.02 .05 .03 /$ SR03 $04.03 .01 /$ SR07 $06.01 .02 / S R 63 \quad 06.01 .02 / S R 64$

\#D $7.3 .1 .12:$

$02.03 .03 /$ SR14 $04.01 .06 / S R 0404.01 .06 / S R 05 \quad 04.01 .06 /$ SR0 6

$04.01 .06 /$ SR0 $704.01 .06 /$ SR0 $04.02 .05 .01 /$ SR0 $404.02 .05 .02 / S R 04$

$04.02 .05 .03 /$ SR03 $04.03 .01 /$ SR07 $06.01 .02 /$ SR63 06.01.02/SR64

\#D $7.3 .1 .13:$

02.03.03/SR17 04.01.07/SR01 04.01.07/SR02 04.01.07/SR03

$04.01 .07 /$ SR0 $404.01 .07 /$ SR05 $04.01 .07 /$ SR11 $04.01 .07 /$ SR12

$04.01 .07 /$ SR13 04.01.07/SR14 09.01.03.01/SR16 09.01.03.02/SR16

\#D $7.3 .1 .14:$

02.03.03/SR17 04.01.07/SR06 04.01.07/SR07 04.01.07/SR08

04.01.07/SR09 04.01.07/SR10 04.01.07/SR11 04.01.07/SR12

$04.01 .07 /$ SR13 04.01.07/SR14 09.01.03.01/SR16 09.01.03.02/SR16

\#D $7.3 .1 .15:$

$04.01 .08 /$ SR01 $04.01 .08 /$ SR02 $04.01 .08 /$ SR03

\#D $7.3 .1 .16:$

$04.01 .08 /$ SR0 $64.01 .08 /$ SR0 $04.01 .08 / S R 08$

\#D $7.3 .2 .1 .1:$

$04.02 .01 .02 /$ SR01 $04.02 .01 .02 /$ SR02 $04.02 .01 .02 / S R 04$

$04.02 .01 .02 /$ SR05

\#D $7.3 .2 \cdot 1.2:$

$04.02 .02 .02 /$ SR01 $04.02 .02 .02 /$ SR02 $04.02 .02 .02 / S R 04$

$04.02 .02 .02 /$ SR0 5

\#D $7.3 .2 \cdot 1.3:$

$04.02 .03 .02 /$ SR01 $04.02 .03 .02 /$ SR0 $204.02 .03 .02 /$ SR0 4

$04.02 .03 .02 /$ SR0 $504.02 .04 .02 /$ SR02 $04.02 .04 .02 /$ SR0 3

\#D $7.3 .2 .1 .4:$

$04.02 .05 .02 /$ SR01 $04.02 .05 .02 /$ SR02 $04.02 .05 .02 /$ SR0 5

$04.02 .05 .02 /$ SR0 6

\#D $7.3 .2 .1 .5:$

$04.02 .05 .03 /$ SR01 $04.02 .05 .03 /$ SR02 $04.02 .05 .03 / S R 04$

$04.02 .05 .03 /$ SR0 5

\#D $7.3 .2 .2 .1:$

$04.02 .01 .01 /$ SR01 $04.02 .01 .01 / S R 02 \quad 04.02 .01 .01 / S R 04$

$04.02 .01 .01 /$ SR0 5

\#D $7.3 .2 .2 .2:$

$04.02 .01 .01 /$ SR01 $04.02 .01 .01 /$ SR02 $04.02 .01 .01 / S R 13$

$04.02 .01 .01 /$ SR14

\#D 7.3.2.2.3:

$04.02 .01 .01 /$ SR01 $04.02 .01 .01 /$ SR02 $04.02 .01 .01 / S R 19$

$04.02 .01 .01 /$ SR2 0 
\#D $7.3 .2 \cdot 3 \cdot 1:$

$04.02 .02 .01 /$ SR01 $04.02 .02 .01 /$ SR02 $04.02 .02 .01 / S R 04$ $04.02 .02 .01 /$ SR0 5

\#D $7.3 .2 \cdot 3 \cdot 2:$

$04.02 .02 .01 /$ SR01 $04.02 .02 .01 /$ SR0 $204.02 .02 .01 / S R 13$ $04.02 .02 .01 / \mathrm{SR} 14$

\#D $7.3 \cdot 2 \cdot 3 \cdot 3:$

$04.02 .02 .01 /$ SR01 $04.02 .02 .01 / S R 0204.02 .02 .01 / S R 20$ $04.02 .02 .01 / \mathrm{SR} 21$

\#D $7.3 \cdot 2 \cdot 4 \cdot 1:$

$04.02 .03 .01 /$ SR09 $04.02 .03 .01 /$ SR10 $04.02 .03 .01 /$ SR12 $04.02 .03 .01 /$ SR13 $04.02 .04 .01 /$ SR0 6

\#D $7.3 \cdot 2 \cdot 4 \cdot 2:$

$04.02 .03 .01 /$ SR09 $04.02 .03 .01 /$ SR10 $04.02 .03 .01 / S R 25$

$04.02 .03 .01 /$ SR2 $604.02 .03 .01 /$ SR2 $704.02 .04 .01 /$ SR 18

$04.02 .04 .01 /$ SR19

\#D $7 \cdot 3 \cdot 2 \cdot 4 \cdot 3:$

$04.02 .03 .01 /$ SR09 $04.02 .03 .01 /$ SR10 $04.02 .03 .01 /$ SR3 4

$04.02 .03 .01 /$ SR35 $04.02 .04 .01 /$ SR2 6

\#D $7.3 .2 .4 .4:$

$04.02 .03 .01 /$ SR09 $04.02 .03 .01 /$ SR10 $04.02 .03 .01 / S R 40$

$04.02 .03 .01 /$ SR $4104.02 .04 .01 /$ SR 32

\#D $7.3 .2 .4 \cdot 5:$

$04.02 .03 .01 /$ SR09 $04.02 .03 .01 /$ SR10 $04.02 .03 .01 /$ SR 46

$04.02 .03 .01 /$ SR $4704.02 .04 .01 /$ SR37

\#D $7.3 .2 .4 .6:$

$02.03 .03 /$ SR15 $04.02 .03 .01 /$ SR09 $04.02 .03 .01 /$ SR10 $04.02 .03 .01 / S R 52$ $04.02 .03 .01 /$ SR58

\#D $7.3 .2 .4 .7:$

$02.03 .03 /$ SR15 $04.02 .03 .01 /$ SR09 $04.02 .03 .01 /$ SR $10 \quad 04.02 .03 .01 / S R 79$ $04.02 .03 .01 /$ SR80

\#D $7.3 .2 .4 .8:$

$02.03 .03 /$ SR15 $04.02 .03 .01 /$ SR09 $04.02 .03 .01 /$ SR10 $04.02 .03 .01 /$ SR64 $04.02 .03 .01 /$ SR $6504.02 .03 .01 /$ SR 66

\#D 7.3.2.4.9:

$02.03 .03 /$ SR15 $04.02 .03 .01 /$ SR09 $04.02 .03 .01 / S R 10 \quad 04.02 .03 .01 / S R 72$ $04.02 .03 .01 /$ SR73 $04.02 .03 .01 /$ SR74

\#D $7.3 .2 .5 .1:$

$04.02 .04 .01 /$ SR03 $04.02 .04 .01 /$ SR0 $404.02 .04 .01 / S R 42$

$04.02 .04 .01 /$ SR 48 
\#D $7.3 \cdot 2 \cdot 5 \cdot 2:$

$04.02 .04 .01 / S R 0304.02 .04 .01 / S R 04 \quad 04.02 .04 .01 / S R 70$ $04.02 .04 .01 /$ SR71

\#D $7 \cdot 3 \cdot 2 \cdot 5 \cdot 3:$

$04.02 .04 .01 /$ SR03 $04.02 .04 .01 /$ SR0 $404.02 .04 .01 /$ SR55

$04.02 .04 .01 /$ SR5 $604.02 .04 .01 /$ SR5 7

\#D $7.3 \cdot 2 \cdot 5 \cdot 4:$

$04.02 .04 .01 / \mathrm{SR} 0304.02 .04 .01 / \mathrm{SR} 0404.02 .04 .01 / \mathrm{SR} 63$

$04.02 .04 .01 /$ SR $6404.02 .04 .01 /$ SR 65

\#D $7.3 \cdot 2 \cdot 5 \cdot 5:$

$04.02 .04 .01 /$ SR03 $04.02 .04 .01 /$ SR0 $404.02 .04 .01 /$ SR75

$04.02 .04 .01 / S R 76$

\#D $7.3 .2 \cdot 6.1:$

$04.02 .05 .01 / \mathrm{SR} 0104.02 .05 .01 / \mathrm{SR} 0204.02 .05 .01 / \mathrm{SR} 05$ $04.02 .05 .01 / \mathrm{SR} 07$

\#D $7.3 .2 .6 .2:$

$04.02 .05 .01 /$ SR01 $04.02 .05 .01 /$ SR02 $04.02 .05 .01 / S R 18$ $04.02 .05 .01 /$ SR19

\#D $7.3 \cdot 2 \cdot 6 \cdot 3:$

$04.02 .05 .01 /$ SR01 $04.02 .05 .01 /$ SR02 $04.02 .05 .01 / S R 26$ $04.02 .05 .01 /$ SR2 7

\#D $7.3 \cdot 2 \cdot 6.4:$

$04.02 .05 .01 /$ SR0 $104.02 .05 .01 /$ SR02 $04.02 .05 .01 / S R 32$

$04.02 .05 .01 /$ SR3 $04.02 .05 .01 /$ SR3 4

\#D $7.3 .2 \cdot 6 \cdot 5:$

$04.02 .05 .01 /$ SR01 $04.02 .05 .01 /$ SR02 $04.02 .05 .01 / S R 40$ $04.02 .05 .01 / \mathrm{SR} 41$

\#D 7.3.2.6.6:

$04.02 .05 .01 /$ SR01 $04.02 .05 .01 /$ SR02 $04.02 .05 .01 / S R 49$ $04.02 .05 .01 / \mathrm{SR} 50$

\#D 7.3.2.6.7:

$04.02 .05 .01 /$ SR0 $104.02 .05 .01 /$ SR02 $04.02 .05 .01 /$ SR55 $04.02 .05 .01 / \mathrm{SR} 56$

\#D $7.3 .2 \cdot 6.8:$

$04.02 .05 .01 /$ SR01 $04.02 .05 .01 /$ SR02 $04.02 .05 .01 / S R 61$

$04.02 .05 .01 /$ SR $6204.02 .05 .01 /$ SR 63

\#D 7.3.2.6.9:

$04.02 .05 .01 /$ SR01 $04.02 .05 .01 /$ SR02 $04.02 .05 .01 / S R 66$ $04.02 .05 .01 /$ SR68 $04.02 .05 .01 /$ SR70

\#D 7.3.2.6.10:

$04.02 .05 .01 /$ SR01 $04.02 .05 .01 /$ SR02 $04.02 .05 .01 /$ SR 67 
$04.02 .05 .01 /$ SR 69

\#D $7.3 \cdot 2 \cdot 7.1:$

$02.03 .03 /$ SR0 $602.03 .03 /$ SR18 $02.03 .03 /$ SR19 $02.03 .03 /$ SR20

$04.03 .04 .01 / \mathrm{SR} 0104.03 .04 .01 / \mathrm{SR} 0204.03 .04 .01 / \mathrm{SR} 03$

$04.03 .04 .01 /$ SR09 $04.03 .04 .01 /$ SR10 $04.03 .04 .01 /$ SR12

$04.03 .04 .01 /$ SR1 6

\#D $7.3 .2 .7 .2:$

$02.03 .03 /$ SR0 $602.03 .03 /$ SR1 $802.03 .03 /$ SR19 $02.03 .03 /$ SR20

$04.03 .04 .01 /$ SR0 $404.03 .04 .01 /$ SR0 $504.03 .04 .01 /$ SR0 6

$04.03 .04 .01 /$ SR0 $904.03 .04 .01 /$ SR10 $04.03 .04 .01 /$ SR13

$04.03 .04 .01 /$ SR1 6

\#D $7.3 .2 .8:$

$04.03 .01 /$ SR01 $04.03 .01 /$ SR02 $04.03 .01 /$ SR03 $04.03 .01 /$ SR0 4 $04.03 .01 /$ SR05 $04.03 .01 /$ SR0 6

\#D $7.3 .2 .9:$

$04.03 .03 /$ SR01 $04.03 .03 /$ SR02 $04.03 .03 /$ SR0 3

\#D $7.3 .2 .10:$

$06.02 .02 /$ SR01 $06.02 .02 /$ SR02 $06.02 .02 /$ SR03

\#D $7 \cdot 3 \cdot 3 \cdot 1:$

$02.03 .03 /$ SR10 $06.01 .01 /$ SR01 $06.01 .01 / S R 02 \quad 06.01 .02 / S R 01$ $06.01 .02 /$ SR02 $06.01 .02 /$ SR03 $06.01 .02 /$ SR0 $706.01 .02 /$ SR0 8 $06.01 .02 /$ SR1 8

\#D $7.3 .3 .2:$

$02.03 .03 /$ SR10 $06.01 .01 /$ SR01 $06.01 .01 /$ SR02 $06.01 .02 /$ SR0 4 $06.01 .02 /$ SR05 $06.01 .02 /$ SR0 $606.01 .02 /$ SR0 $706.01 .02 /$ SR1 8

\#D $7 \cdot 3 \cdot 3 \cdot 3:$

$02.03 .03 /$ SR10 $06.01 .01 /$ SR01 $06.01 .01 /$ SR02 $06.01 .02 /$ SR0 9 $06.01 .02 / S R 10 \quad 06.01 .02 / S R 1106.01 .02 / S R 1506.01 .02 / S R 18$

\#D $7.3 .3 .4:$

$02.03 .03 /$ SR10 $06.01 .01 /$ SR01 $06.01 .01 /$ SR02 $06.01 .02 / S R 12$ $06.01 .02 /$ SR13 $06.01 .02 /$ SR14 $06.01 .02 /$ SR18

\#D $7 \cdot 3 \cdot 3 \cdot 5:$

$02.03 .03 /$ SR10 $06.01 .02 / S R 2306.01 .02 / S R 24 \quad 06.01 .02 / S R 25$

$06.01 .02 /$ SR38 $06.01 .02 /$ SR39 $06.01 .02 /$ SR40 $06.01 .02 /$ SR4 1

$06.01 .02 /$ SR42 06.01 .02 /SR43 $06.01 .02 /$ SR4 $406.01 .02 /$ SR 45

$06.01 .02 / S R 46 \quad 06.01 .02 / S R 47 \quad 06.01 .02 / S R 48 \quad 06.01 .02 / S R 49$

\#D $7.3 \cdot 3 \cdot 6:$

$02.03 .03 /$ SR10 $06.01 .02 /$ SR2 $606.01 .02 / S R 27 \quad 06.01 .02 / S R 28$ $06.01 .02 /$ SR3 $86.01 .02 /$ SR39 $06.01 .02 /$ SR 49

\#D $7.3 \cdot 3 \cdot 7:$

$06.01 .02 / \mathrm{SR} 2906.01 .02 / \mathrm{SR} 30 \quad 06.01 .02 / \mathrm{SR} 31 \quad 06.01 .02 / \mathrm{SR} 52$ $06.01 .02 / \mathrm{SR} 53$ 
\#D $7.3 .3 .8:$

$02.03 .03 /$ SR10 $06.01 .02 /$ SR32 $06.01 .02 /$ SR33 $06.01 .02 /$ SR34

$06.01 .02 / \mathrm{SR} 56$

\#D $7.3 .4:$

$02.01 /$ SR01 $02.01 / S R 0302.01 / S R 0402.01 / S R 0502.01 / S R 0602.01 / S R 07$

$02.01 /$ SR0 $802.01 / S R 0902.01 /$ SR10 $02.01 /$ SR11 $02.01 /$ SR12 $02.01 / S R 13$

$02.01 /$ SR14 02.01.02.01/SR02 02.01.02.01/SR06 02.01.02.01/SR07

$02.01 .02 .02 /$ SR01 $02.01 .02 .02 /$ SR02 $02.01 .02 .02 / S R 03$

$02.01 .02 .02 /$ SR0 $402.01 .02 .02 /$ SR0 $502.01 .02 .02 /$ SR0 6

$02.01 .02 .02 /$ SR0 $702.01 .02 .02 /$ SR0 $802.01 .02 .02 / S R 11$

$02.01 .02 .02 /$ SR12 $02.01 .03 .01 /$ SR01 $02.01 .03 .02 / S R 01$

$02.01 .03 .02 / \mathrm{SR} 0202.01 .03 .02 / \mathrm{SR} 03 \quad 02.01 .03 .02 / \mathrm{SR} 04$

$02.01 .03 .03 /$ SR01 $02.01 .03 .03 /$ SR0 $702.01 .03 .03 /$ SR0 $02.02 .04 / S R 10$

$02.03 .03 /$ SR0 $83.03 /$ SR02 $03.03 /$ SR19 $03.04 /$ SR0 3

\#D $7.3 .5:$

$02.02 .02 /$ SR01 $02.02 .02 /$ SR02 $02.02 .02 /$ SR03 $02.02 .03 /$ SR0 9

$02.02 .04 /$ SR0 $502.02 .04 /$ SR0 $609.01 .05 .01 /$ SR03 $09.01 .05 .01 /$ SR0 4

$09.01 .05 .02 /$ SR03 $09.01 .05 .02 /$ SR0 4

\#D $7.3 .6:$

$02.02 .02 / \mathrm{SR} 0402.02 .02 / \mathrm{SR} 05 \quad 02.02 .02 / \mathrm{SR} 06$

\#D $7.3 .7:$

$02.02 .02 /$ SR0 $702.02 .02 /$ SR0 $802.02 .02 /$ SR09 $02.02 .02 /$ SR10

\#D $8:$

$09.01 / \mathrm{SR} 04$

\#D $8.1:$

09.01/SR04 09.01.01.01/SR01 09.01.01.01/SR02 09.01.01.01/SR03

09.01.01.01/SR04 09.01.01.01/SR05 09.01.01.01/SR0 6

09.01.01.01/SR07 09.01.01.02/SR01 09.01.01.02/SR02

09.01.01.02/SR03 09.01.01.02/SR04 09.01.01.02/SR0 5

09.01.01.02/SR06 09.01.01.02/SR07 09.01.02.01/SR01

09.01.02.01/SR02 09.01.02.01/SR03 09.01.02.01/SR0 4

$09.01 .02 .01 /$ SR05 $09.01 .02 .01 /$ SR0 $609.01 .02 .01 / S R 07$

$09.01 .02 .01 /$ SR08 $09.01 .02 .01 /$ SR09 $09.01 .02 .01 /$ SR 10

09.01.02.01/SR11 $09.01 .02 .01 /$ SR12 $09.01 .02 .02 /$ SR01

09.01.02.02/SR02 09.01.02.02/SR03 09.01.02.02/SR0 4

$09.01 .02 .02 /$ SR0 $509.01 .02 .02 /$ SR0 $609.01 .02 .02 / S R 07$

$09.01 .02 .02 /$ SR08 $09.01 .02 .02 /$ SR09 $09.01 .02 .02 / S R 10$

$09.01 .02 .02 /$ SR11 $09.01 .02 .02 / S R 12 \quad 09.01 .03 .01 / S R 01$

$09.01 .03 .01 /$ SR02 $09.01 .03 .01 /$ SR03 $09.01 .03 .01 / S R 04$

$09.01 .03 .01 /$ SR05 $09.01 .03 .01 /$ SR0 $609.01 .03 .01 / S R 07$

$09.01 .03 .01 /$ SR08 $09.01 .03 .01 /$ SR09 $09.01 .03 .01 / S R 10$

$09.01 .03 .01 /$ SR11 $09.01 .03 .01 /$ SR12 $09.01 .03 .01 /$ SR13

$09.01 .03 .01 /$ SR14 $09.01 .03 .01 /$ SR15 $09.01 .03 .01 /$ SR1 6

$09.01 .03 .01 /$ SR17 $09.01 .03 .02 /$ SR01 $09.01 .03 .02 /$ SR02

$09.01 .03 .02 /$ SR03 $09.01 .03 .02 /$ SR0 $409.01 .03 .02 / S R 05$

$09.01 .03 .02 /$ SR0 $609.01 .03 .02 /$ SR0 $709.01 .03 .02 / S R 08$

$09.01 .03 .02 /$ SR09 $09.01 .03 .02 /$ SR10 09.01.03.02/SR11 
$09.01 .03 .02 / \mathrm{SR} 12$ $09.01 .03 .02 /$ SR15 $09.01 .04 .01 / \mathrm{SR} 01$ $09.01 .04 .01 /$ SR0 4 $09.01 .04 .01 /$ SR07 $09.01 .04 .02 / \mathrm{SR} 03$ $09.01 .04 .02 /$ SR0 6 $09.01 .05 .01 / \mathrm{SR} 02$ $09.01 .05 .01 / \mathrm{SR} 05$ $09.01 .05 .02 / \mathrm{SR} 02$ $09.01 .05 .02 / \mathrm{SR} 05$ $09.01 .09 .01 / \mathrm{SR} 02$ $09.01 .09 .01 /$ SR05 $09.01 .09 .01 /$ SR0 8 $09.01 .09 .02 / \mathrm{SR} 02$ 09.01 .09 .02 /SR05 $09.01 .09 .02 /$ SR0 8
$09.01 .03 .02 /$ SR13 $09.01 .03 .02 /$ SR1 6 $09.01 .04 .01 /$ SR02 $09.01 .04 .01 /$ SR0 5 $09.01 .04 .02 /$ SR01 09.01 .04 .02 /SR0 4 09.01 .04 .02 /SR0 7 $09.01 .05 .01 /$ SR0 3 $09.01 .05 .01 /$ SR0 6 $09.01 .05 .02 /$ SR0 3 09.01 .05 .02 /SR0 6 $09.01 .09 .01 /$ SR03 $09.01 .09 .01 /$ SR0 6 $09.01 .09 .01 /$ SRO 9 09.01 .09 .02 /SR0 3 09.01 .09 .02 /SR0 6 09.01 .09 .02 /SR0 9

$09.01 .03 .02 / S R 14$ $09.01 .03 .02 /$ SR1 7 $09.01 .04 .01 / \mathrm{SR} 03$ $09.01 .04 .01 /$ SR0 6 $09.01 .04 .02 / \mathrm{SR} 02$ $09.01 .04 .02 / \mathrm{SR} 05$ $09.01 .05 .01 /$ SR01 $09.01 .05 .01 / \mathrm{SR} 04$ $09.01 .05 .02 / \mathrm{SR} 01$ $09.01 .05 .02 /$ SR0 4 $09.01 .09 .01 /$ SR01 $09.01 .09 .01 /$ SR0 4 $09.01 .09 .01 / \mathrm{SR} 07$ $09.01 .09 .02 /$ SR01 $09.01 .09 .02 /$ SR0 4 $09.01 .09 .02 /$ SR0 7

\#D 8.2 :

09.01/SR01 09.01/SR02 09.01/SR03 09.01/SR0 $409.01 /$ SR07 09.01/SR10

\#D $8.3:$

$09.01 /$ SR0 $409.01 /$ SR0 9

\#D 9.1 :

03.01/SR07 09.01.05.01/SR01 09.01.05.01/SR02 09.01.05.01/SR05 $09.01 .05 .02 /$ SR01 09.01.05.02/SR02 09.01.05.02/SR05 $09.01 .09 .01 /$ SR01 09.01.09.01/SR02 09.01.09.01/SR03 09.01.09.01/SR04 09.01.09.01/SR05 09.01.09.01/SR06 09.01.09.01/SR07 09.01.09.01/SR08 09.01.09.01/SR09 09.01.09.02/SR01 09.01.09.02/SR02 09.01.09.02/SR03 $09.01 .09 .02 /$ SR0 $409.01 .09 .02 /$ SR05 $09.01 .09 .02 /$ SR0 6 09.01.09.02/SR07 09.01.09.02/SR08 09.01.09.02/SR09

\#D 9.1.1:

03.03/SR0 4 03.03/SR05 03.03/SR0 6 03.03/SR07 03.03/SR0 8 03.03/SR10 $03.03 /$ SR1 $103.03 /$ SR12 $03.03 / S R 13 \quad 03.03 / S R 14 \quad 03.03 / S R 1503.03 / S R 16$ 03.03/SR17 03.04/SR01 03.04/SR02 03.04/SR03 03.04/SR04 03.04/SR0 5 03.04/SR0 $603.04 /$ SR0 $703.04 / S R 08$ 03.04/SR09 03.04/SR10 03.04/SR11 $03.04 /$ SR12 $03.04 /$ SR13 03.04/SR14 03.04/SR15 03.04/SR16 03.04/SR17 $03.05 /$ SR0 $103.05 /$ SR02 $03.05 /$ SR03 $03.05 /$ SR0 4 03.05/SR05 $09.01 .05 .01 /$ SR02 $09.01 .05 .01 /$ SR05 $09.01 .05 .02 /$ SR02 09.01 .05 .02 /SR05 09.01.09.01/SR08 09.01.09.01/SR09 $09.01 .09 .02 /$ SR08 09.01.09.02/SR09

\#D $9.1 .2:$

09.01.05.01/SR02 09.01.05.01/SR05 09.01.05.02/SR02 $09.01 .05 .02 /$ SR0 5

\#D $9.1 .2 .4:$

03.03/SR10 03.04/SR0 4 03.04/SR05 03.04/SR0 6 03.04/SR07 03.04/SR0 8 03.04/SR0 9 03.04/SR10 03.04/SR11 03.04/SR12 03.04/SR13 03.04/SR14 $03.04 /$ SR15 $03.04 /$ SR1 $603.04 /$ SR17 $03.05 /$ SR02 


\section{APPENDIX K}

\section{STANDARD SPECIFICATIONS CROSS-REEERENCE}

\#S $4.1 .1 / 15 / 1$ :

$04.02 .01 .01 /$ SR10 $04.02 .01 .02 /$ SR38 $04.02 .02 .01 / S R 10$

$04.02 .02 .02 /$ SR3 $84.02 .03 .01 / S R 21 \quad 04.02 .03 .02 / S R 40$

$04.02 .04 .01 /$ SR14 $04.02 .04 .01 /$ SR82 $04.02 .04 .02 /$ SR13

$04.02 .05 .01 /$ SR11 $04.02 .05 .01 /$ SR23 $04.02 .05 .01 / S R 46$

$04.02 .05 .02 / S R 4904.02 .05 .03 / S R 4104.03 .02 .02 / S R 29$

\#S $4.1 .2 / 15 / 2$ :

$04.02 .01 .01 /$ SR09 $04.02 .01 .02 /$ SR37 $04.02 .02 .01 /$ SR0 9

$04.02 .02 .02 /$ SR $37 \quad 04.02 .03 .01 /$ SR21 $04.02 .03 .02 / S R 40$

$04.02 .04 .01 /$ SR14 $04.02 .04 .01 /$ SR81 $04.02 .04 .02 /$ SR13

$04.02 .05 .01 / \mathrm{SR} 2204.02 .05 .01 / \mathrm{SR} 4504.02 .05 .03 / \mathrm{SR} 40$

\#S $4.4 .1 / 22 / 1:$

$02.01 .01 /$ SR09

\#S 4.4.1/22/3:

$04.01 .01 /$ SR01 $04.01 .01 /$ SR0 $404.01 .02 /$ SR01 $04.01 .02 /$ SR0 4

$04.01 .03 /$ SR01 $04.01 .03 /$ SR0 $404.01 .04 /$ SR0 $104.01 .04 / S R 04$

$04.01 .05 /$ SR01 $04.01 .05 /$ SR0 $404.01 .06 /$ SR01 $04.01 .06 /$ SR0 4

$04.01 .07 /$ SR01 $04.01 .07 /$ SR0 $604.01 .08 /$ SR01 $04.01 .08 /$ SR0 6

\#S $4.4 .1 / 23 / 1:$

$04.02 .01 .01 /$ SR0 $404.02 .01 .01 /$ SR13 $04.02 .01 .01 /$ SR 19

$04.02 .01 .02 /$ SR0 $404.02 .01 .02 /$ SR0 $504.02 .02 .01 /$ SR0 4

$04.02 .02 .01 /$ SR13 $04.02 .02 .01 /$ SR20 $04.02 .02 .02 /$ SR0 4

$04.02 .02 .02 /$ SR0 $504.02 .03 .01 /$ SR12 $04.02 .03 .01 /$ SR2 5

$04.02 .03 .01 /$ SR34 $04.02 .03 .01 /$ SR40 $04.02 .03 .01 / S R 46$

$04.02 .03 .01 /$ SR52 $04.02 .03 .01 /$ SR64 $04.02 .03 .01 /$ SR72

$04.02 .03 .01 /$ SR79 $04.02 .03 .02 /$ SR0 $404.02 .03 .02 /$ SR0 5

$04.02 .04 .01 /$ SR4 $204.02 .04 .01 /$ SR55 $04.02 .04 .01 /$ SR 63

$04.02 .04 .01 /$ SR70 $04.02 .04 .01 /$ SR75 $04.02 .04 .02 /$ SR0 2

$04.02 .04 .02 /$ SR03 $04.02 .05 .01 /$ SR0 $504.02 .05 .01 /$ SR18

$04.02 .05 .01 /$ SR26 $04.02 .05 .01 /$ SR32 $04.02 .05 .01 /$ SR 40

$04.02 .05 .01 /$ SR $4904.02 .05 .01 /$ SR55 $04.02 .05 .01 /$ SR 61

$04.02 .05 .01 /$ SR6 $64.02 .05 .01 /$ SR $6704.02 .05 .02 /$ SR0 5

$04.02 .05 .02 /$ SR0 $604.02 .05 .03 /$ SR0 $404.02 .05 .03 /$ SR0 $504.03 .03 / S R 01$

$04.03 .04 .01 /$ SR01 $04.03 .04 .01 /$ SR0 4 
\#S $4.4 .2 / 24 / 3:$

$02.01 /$ SR01

\#S $4.4 .2 / 25 / 2:$

02.01.01/SR01 02.01.01/SR02. 02.01.01/SR03 02.01.01/SR0 4

$02.01 .01 /$ SR05 02.01.01/SR0 $62.01 .01 /$ SR0 $02.01 .01 / S R 08$

$02.01 .01 /$ SR10 $03.03 /$ SR19

\#S $4.4 .2 / 25 / 3$ :

$02.01 .02 .01 / \mathrm{SR} 03$

\#S $4.4 .3 / 25 / 4$ :

$05.02 /$ SR10 05.03/SR01 $05.03 /$ SR02

\#S $4.4 .3 / 25 / 5$ :

$02.01 .01 / \mathrm{SR} 0405.03 / \mathrm{SR} 0505.03 / \mathrm{SR} 06 \quad 05.03 / \mathrm{SR} 07 \quad 05.03 / \mathrm{SR} 08$ $05.03 /$ SR0 9

\#S $4.4 .3 / 25 / 8:$

$05.03 /$ SR03 $05.03 / S R 0405.03 / S R 15$

\#S $4.4 .3 / 26 / 2$ :

$04.02 .01 .01 /$ SR11 $04.02 .01 .01 /$ SR17 $04.02 .01 .01 / S R 23$

$04.02 .01 .02 /$ SR0 $804.02 .02 .01 /$ SR11 $04.02 .02 .01 /$ SR1 8

$04.02 .02 .01 /$ SR2 $404.02 .02 .02 /$ SR0 $804.02 .03 .01 / S R 23$

$04.02 .03 .01 / \mathrm{SR} 32.04 .02 .03 .01 / \mathrm{SR} 38 \quad 04.02 .03 .01 / \mathrm{SR} 44$

$04.02 .03 .01 /$ SR50 $04.02 .03 .01 /$ SR62 $04.02 .03 .01 / S R 70$

$04.02 .03 .01 / S R 7704.02 .03 .01 /$ SR82 $04.02 .03 .02 /$ SR0 8

$04.02 .04 .01 /$ SR1 $604.02 .04 .01 /$ SR2 4 04.02.04.01/SR30

$04.02 .04 .01 /$ SR35 $04.02 .04 .01 /$ SR40 $04.02 .04 .01 / S R 53$

$04.02 .04 .01 /$ SR61 $04.02 .04 .01 /$ SR68 $04.02 .04 .01 / \mathrm{SR} 73$

$04.02 .04 .01 /$ SR83 $04.02 .04 .02 /$ SR0 $604.02 .05 .01 /$ SR 16

$04.02 .05 .01 / S R 2404.02 .05 .01 /$ SR30 $04.02 .05 .01 / S R 38$

$04.02 .05 .01 / S R 4704.02 .05 .01 /$ SR53 $04.02 .05 .01 / S R 59$

$04.02 .05 .01 /$ SR $6404.02 .05 .01 /$ SR80 $04.02 .05 .01 /$ SR 82

$04.02 .05 .02 /$ SR0 $94.02 .05 .03 /$ SR0 $804.03 .01 /$ SR10 $06.01 .02 /$ SR 19

$06.01 .02 /$ SR50 $06.01 .02 /$ SR54

\#S $4.4 .3 / 28 / 1:$

$04.02 .01 .01 /$ SR11 $04.02 .01 .01 /$ SR12 $04.02 .01 .01 / S R 17$

$04.02 .01 .01 /$ SR18 $04.02 .01 .01 /$ SR23 $04.02 .01 .01 /$ SR2 4

$04.02 .01 .02 /$ SR0 $804.02 .01 .02 /$ SR0 $904.02 .02 .01 / S R 11$

$04.02 .02 .01 /$ SR12 $04.02 .02 .01 /$ SR1 8 04.02.02.01/SR1 9

$04.02 .02 .01 /$ SR2 $404.02 .02 .01 /$ SR2 $504.02 .02 .02 /$ SR0 8

$04.02 .02 .02 /$ SR09 $04.02 .03 .01 / S R 2304.02 .03 .01 / S R 24$

$04.02 .03 .01 /$ SR32 $04.02 .03 .01 /$ SR33 $04.02 .03 .01 /$ SR3 8

$04.02 .03 .01 /$ SR39 $04.02 .03 .01 /$ SR4 $404.02 .03 .01 / S R 45$

$04.02 .03 .01 /$ SR50 $04.02 .03 .01 /$ SR51 $04.02 .03 .01 / S R 62$

$04.02 .03 .01 / \mathrm{SR} 63 \quad 04.02 .03 .01 / \mathrm{SR} 70 \quad 04.02 .03 .01 / \mathrm{SR} 71$

$04.02 .03 .01 /$ SR77 $04.02 .03 .01 /$ SR78 $04.02 .03 .01 /$ SR8 2

$04.02 .03 .01 /$ SR8 $304.02 .03 .02 /$ SR0 $804.02 .03 .02 /$ SR0 9

$04.02 .04 .01 /$ SR1 $604.02 .04 .01 /$ SR17 $04.02 .04 .01 / S R 24$

$04.02 .04 .01 / S R 2504.02 .04 .01 /$ SR30 $04.02 .04 .01 / S R 31$ 
$04.02 .04 .01 / \mathrm{SR} 3504.02 .04 .01 / \mathrm{SR} 3604.02 .04 .01 / \mathrm{SR} 40$

$04.02 .04 .01 /$ SR4 $104.02 .04 .01 /$ SR53 $04.02 .04 .01 /$ SR5 4

$04.02 .04 .01 /$ SR61 $04.02 .04 .01 /$ SR62 $04.02 .04 .01 /$ SR 68

$04.02 .04 .01 /$ SR69 $04.02 .04 .01 /$ SR73 $04.02 .04 .01 / S R 74$

$04.02 .04 .01 /$ SR83 $04.02 .04 .01 /$ SR8 $404.02 .04 .02 /$ SR0 6

$04.02 .04 .02 /$ SR0 $704.02 .05 .01 /$ SR16 $04.02 .05 .01 /$ SR17

$04.02 .05 .01 / \mathrm{SR} 2404.02 .05 .01 / \mathrm{SR} 25 \quad 04.02 .05 .01 / \mathrm{SR} 30$

$04.02 .05 .01 /$ SR31 $04.02 .05 .01 /$ SR3 $84.02 .05 .01 /$ SR3 9

$04.02 .05 .01 / \mathrm{SR} 4704.02 .05 .01 / \mathrm{SR} 4804.02 .05 .01 / \mathrm{SR} 53$

$04.02 .05 .01 /$ SR5 $404.02 .05 .01 /$ SR5 $904.02 .05 .01 /$ SR 60

$04.02 .05 .01 /$ SR64 $04.02 .05 .01 /$ SR65 $04.02 .05 .01 /$ SR8 0

$04.02 .05 .01 /$ SR81 $04.02 .05 .01 /$ SR8 $204.02 .05 .01 /$ SR8 3

$04.02 .05 .02 /$ SR09 $04.02 .05 .02 /$ SR10 $04.02 .05 .03 /$ SR0 8

$04.02 .05 .03 /$ SR09 $04.03 .01 /$ SR10 $04.03 .01 /$ SR11 $04.03 .04 .01 / \mathrm{SR} 14$

$06.01 .02 /$ SR19 $06.01 .02 /$ SR20 $06.01 .02 /$ SR50 $06.01 .02 /$ SR5 1

$06.01 .02 /$ SR5 $406.01 .02 /$ SR55

\#S $4.4 .4 / 28 / 4:$

$02.03 .02 / \mathrm{SR} 0402.03 .02 / \mathrm{SR} 05$

\#S $4.4 .4 / 28 / 5$ :

$02.01 .01 /$ SR01 $02.02 .01 /$ SR01 $02.02 .01 /$ SR02 $02.02 .01 / S R 04$

$02.02 .01 /$ SR05 $02.02 .01 /$ SR0 6

\#S $4.4 .4 / 28 / 6$ :

$02.02 .01 /$ SR03 $02.02 .01 /$ SR0 8

\#S $4.4 .4 / 28 / 7$ :

$02.02 .02 /$ SR01 $02.02 .03 /$ SR07 $02.02 .03 /$ SR0 $02.02 .03 /$ SR0 9

$02.02 .03 /$ SR 10

\#S $4.4 .4 / 29 / 1$ :

$02.02 .03 /$ SR01 $02.02 .03 /$ SR02 $02.02 .03 /$ SR03 $02.02 .03 /$ SR0 4

\#S $4.4 .4 / 29 / 2:$

$02.02 .05 /$ SR01 $02.02 .05 / S R 0202.02 .05 / S R 0302.02 .05 /$ SR0 4

\#S $4.4 .4 / 29 / 3$ :

$02.02 .04 /$ SR01 $02.02 .04 /$ SR02 $02.02 .04 /$ SR03 $02.02 .04 / S R 04$

$02.02 .04 /$ SR0 $52.02 .04 /$ SR0 $02.02 .04 /$ SR0 7

\#S $4.4 .4 / 29 / 4:$

$02.01 .01 /$ SR02 $02.02 .04 /$ SR08 $02.02 .04 /$ SR09 $02.02 .04 / S R 10$ $02.02 .04 /$ SR11

\#S $4.4 .4 / 29 / 5$ :

$02.02 .02 / \mathrm{SR} 02$

$02.02 .02 /$ SR0 9

$02.02 .02 / \mathrm{SR} 0302.02 .02 / \mathrm{SR} 05$

$02.02 .02 / S R 1002.03 .01 /$ SR01

$02.02 .02 / \mathrm{SR} 06$

$02.03 .01 / \mathrm{SR} 03$

$04.01 .01 /$ SR02 $04.01 .01 /$ SR03

$04.01 .01 /$ SRO 6

$04.01 .02 /$ SR02 $04.01 .02 /$ SR03

$04.01 .02 /$ SR0 6

$04.01 .03 /$ SR02 $04.01 .03 /$ SR0 3

$04.01 .03 /$ SR0 6

$04.01 .04 / \mathrm{SR} 02$

$04.01 .04 / \mathrm{SR} 03$

$04.01 .04 /$ SR0 6

$04.01 .05 / \mathrm{SR} 02$

$04.01 .05 /$ SR0 3

$04.01 .05 /$ SR0 6

$04.01 .06 /$ SR02

$04.01 .06 /$ SR0 3

$02.03 .01 / S R 02$

$04.01 .01 / \mathrm{SR} 05$

$04.01 .02 /$ SR0 5

$04.01 .03 /$ SR0 5

$04.01 .04 /$ SR0 5

$04.01 .05 /$ SR0 5

$04.01 .06 / S R 05$ 
$04.01 .06 /$ SR0 $64.01 .07 / S R 02 \quad 04.01 .07 / S R 03 \quad 04.01 .07 / S R 07$

$04.01 .07 /$ SR0 $804.01 .08 /$ SR02 $04.01 .08 /$ SR03 $04.01 .08 /$ SR0 7

$04.01 .08 /$ SR0 $804.02 .01 .01 /$ SR01 $04.02 .01 .01 /$ SR02 $04.02 .01 .02 / S R 01$

$04.02 .01 .02 /$ SR02 $04.02 .02 .01 /$ SR01 $04.02 .02 .01 / S R 02$

$04.02 .02 .02 /$ SR01 $04.02 .02 .02 /$ SR02 $04.02 .03 .01 /$ SR0 9

$04.02 .03 .01 /$ SR10 $04.02 .03 .02 /$ SR01 $04.02 .03 .02 /$ SR02

$04.02 .04 .01 /$ SR03 $04.02 .04 .01 /$ SR0 $404.02 .05 .01 / S R 01$

$04.02 .05 .01 /$ SR02 $04.02 .05 .02 /$ SR01 $04.02 .05 .02 / S R 02$

$04.02 .05 .03 /$ SR01 $04.02 .05 .03 /$ SR02 $04.03 .01 / S R 0204.03 .01 / S R 03$

$04.03 .03 /$ SR02 $04.03 .03 /$ SR03 $04.03 .04 .01 /$ SR02 $04.03 .04 .01 / S R 03$

$04.03 .04 .01 /$ SR0 $504.03 .04 .01 /$ SR0 $606.01 .02 / S R 0206.01 .02 / S R 03$

$06.01 .02 /$ SR05 $06.01 .02 /$ SR0 $606.01 .02 /$ SR10 $06.01 .02 /$ SR11

$06.01 .02 /$ SR13 $06.01 .02 /$ SR14 $06.01 .02 / S R 2406.01 .02 / S R 25$

$06.01 .02 /$ SR27 $06.01 .02 /$ SR28 $06.01 .02 /$ SR30 $06.01 .02 / S R 31$

$06.01 .02 / \mathrm{SR} 3306.01 .02 / \mathrm{SR} 3406.02 .02 / \mathrm{SR} 0206.02 .02 / \mathrm{SR} 03$

\#S $4.4 .5 / 29 / 7$ :

$02.01 .02 .01 /$ SR01 $02.01 .02 .01 /$ SR02 $02.01 .02 .01 / S R 06$

$02.01 .02 .01 /$ SR07 $02.01 .02 .02 /$ SR11 $02.01 .02 .02 / S R 12$

$02.01 .02 .03 /$ SR01 $02.01 .02 .03 /$ SR02

\#S $4.4 .5 / 29 / 8$ :

$02.01 .02 .02 /$ SR01 $02.01 .02 .02 / S R 02 \quad 02.01 .02 .02 / S R 03$

$02.01 .02 .02 /$ SR0 $402.01 .02 .02 /$ SR0 $5 \quad 02.01 .02 .02 /$ SR0 6

$02.01 .02 .02 /$ SR0 $702.01 .02 .02 /$ SR0 $802.01 .02 .02 /$ SR0 9

$02.01 .02 .02 /$ SR11 $02.01 .02 .02 /$ SR12

\#S $4.4 .5 / 29 / 9:$

$02.01 .02 .01 / \mathrm{SR} 0402.01 .02 .02 / \mathrm{SR} 10$

\#S $4.4 .5 / 30 / 1$ :

$02.01 .01 /$ SR05 $02.01 .01 /$ SR07 $02.01 .03 .01 /$ SR01 $02.01 .03 .01 / S R 02$

$02.01 .03 .01 /$ SR03 $02.01 .03 .02 /$ SR01 $02.01 .03 .02 / S R 02$

$02.01 .03 .02 /$ SR03 $02.01 .03 .02 /$ SR0 $402.01 .03 .02 /$ SR0 5

$02.01 .03 .02 /$ SR0 $62.01 .03 .03 /$ SR01 $02.01 .03 .03 / S R 02$

$02.01 .03 .03 /$ SR0 $302.01 .03 .03 /$ SR0 $02.01 .03 .03 /$ SR0 8

\#S $4.4 .5 / 30 / 2:$

$02.01 /$ SR02 $02.01 .01 /$ SR09

\#S $4.4 .6 / 30 / 4:$

$02.03 .01 /$ SR0 $402.03 .01 /$ SR0 $02.03 .01 /$ SR0 6

\#S $4.4 .6 / 30 / 5:$

$02.01 /$ SR03 02.01/SR04 02.01/SR0 $502.01 /$ SR0 $602.01 / S R 0902.01 / S R 10$ $02.01 /$ SR11 $02.01 /$ SR12 $02.01 /$ SR13 $02.01 /$ SR1 4

\#S $4.4 .6 / 30 / 6:$

$02.03 .02 /$ SR01 $02.03 .02 /$ SR02 $02.03 .02 /$ SR03 $02.03 .02 / S R 04$

$02.03 .02 /$ SR0 $502.03 .02 /$ SR0 $602.03 .02 /$ SR0 $02.03 .02 /$ SR0 8

$02.03 .02 /$ SR09

\#S $4.4 .6 / 30 / 9$ :

$02.03 .03 /$ SR02 $02.03 .03 /$ SR03 $02.03 .03 /$ SR0 $402.03 .03 /$ SR0 6 
$02.03 .03 / S R 07$

\#S $4.4 .6 / 31 / 1$ :

$02.03 .03 /$ SR0 $602.03 .03 /$ SR0 9

\#S $4.4 .6 / 31 / 2$ :

$02.03 .03 /$ SR15

\#S $4.4 .6 / 31 / 3$ :

$02.03 .03 / \mathrm{SR} 16$

\#S $4.4 .6 / 31 / 4$ :

$02.03 .03 / \mathrm{SR0} 4$

\#S $4.4 .6 / 31 / 5$ :

$02.03 .03 /$ SR0 $402.03 .03 / \mathrm{SR} 08$

\#S $4.4 .6 / 31 / 6$ :

$02.03 .03 /$ SR0 5

\#S $4.4 .6 / 31 / 9$ :

$02.03 .03 /$ SR 20

\#S $4.4 .6 / 31 / 11$ :

$02.03 .03 /$ SR18 $02.03 .03 /$ SR 19

\#S $4.4 .7 / 32 / 3$ :

$03.03 /$ SR0 $103.03 /$ SR02 $03.03 /$ SR03 $03.03 /$ SR0 $903.03 /$ SR10 $03.03 /$ SR1 1

$03.03 /$ SR12 03.05/SR0 $103.05 /$ SR02 $03.05 /$ SR03

\#S $4.4 .7 / 32 / 4$ :

03.01/SR01 03.01/SR07 03.03/SR04 03.05/SR0 $403.05 /$ SR0 5

\#S $4.4 .7 / 32 / 5$ :

03.01/SR05 $03.01 /$ SR0 $803.01 /$ SR10 $03.01 /$ SR11 $03.04 /$ SR01

\#S $4.4 .7 / 32 / 6$ :

03.04/SR0 $503.04 /$ SR0 $603.04 / S R 0703.04 / S R 08 \quad 03.04 / S R 1203.04 / S R 13$

$03.04 /$ SR1 $403.04 /$ SR15 $03.04 /$ SR16 $03.04 /$ SR17

\#S $4.4 .7 / 32 / 7$ :

03.02/SR02 03.02/SR03 03.03/SR0 $03.03 /$ SR0 $703.03 /$ SR0 8

\#S $4.4 .7 / 33 / 1$ :

$03.03 /$ SR0 $603.03 /$ SR0 $703.03 /$ SR0 $803.03 / S R 14 \quad 03.03 /$ SR15 03.03/SR1 6 $03.03 /$ SR 17

\#S $4.4 .7 / 33 / 2$ :

$03.04 / \mathrm{SR} 0203.04 / \mathrm{SR} 0303.04 / \mathrm{SR} 04$

\#S $4.4 .8 / 33 / 3$ :

$02.02 .02 /$ SR0 7

\#S $4.4 .9 / 33 / 4$ : 
$02.02 .02 / \mathrm{SR} 04$

\#S $4.5 .1 / 34 / 1$ :

$02.03 .03 /$ SR11 $02.03 .03 /$ SR12 $02.03 .03 /$ SR13 $02.03 .03 /$ SR14

$02.03 .03 /$ SR15 $02.03 .03 /$ SR1 $602.03 .03 /$ SR17 $04.01 /$ SR01

04.01.01/SR01 04.01.01/SR04 04.01.01/SR07 04.01.02/SR01

04.01.02/SR0 4 04.01.02/SR07 04.01.03/SR01 04.01.03/SR0 4

$04.01 .03 /$ SR09 $04.01 .03 /$ SR10 04.01.03/SR11 04.01.04/SR01

04.01.04/SR0 4 04.01.04/SR07 04.01.04/SR08 04.01.04/SR09

04.01.05/SR01 04.01.05/SR04 04.01.05/SR07 04.01.06/SR01

$04.01 .06 /$ SR0 $404.01 .06 /$ SR0 $704.01 .07 /$ SR01 $04.01 .07 /$ SR0 6

$04.01 .08 / \mathrm{SR} 0104.01 .08 / \mathrm{SR} 0604.02 .05 .01 / \mathrm{SR} 10 \quad 04.02 .05 .01 / \mathrm{SR} 13$

$04.02 .05 .01 /$ SR14 $04.02 .05 .01 /$ SR15

\#S $4.5 .1 / 35 / 1$ :

$02.03 .03 / \mathrm{SR} 1504.02 .03 .01 / \mathrm{SR} 0204.02 .04 .01 / \mathrm{SR} 01$

\#S $4.5 .1 / 35 / 2$ :

$04.01 .03 /$ SR0 8

\#S $4.5 .1 / 35 / 4$ :

$02.03 .03 /$ SR13 $02.03 .03 /$ SR1 4

\#S $4.5 .1 / 35 / 6$ :

$02.03 .03 / \mathrm{SR} 1302.03 .03 / \mathrm{SR} 1404.01 .05 / \mathrm{SR} 08$ 04.01.06/SR0 8

\#S $4.5 .1 / 35 / 7$ :

$02.03 .03 /$ SR13 $02.03 .03 /$ SR14 $06.01 .02 /$ SR 64

\#S $4.5 .1 / 36 / 1:$

$02.03 .03 /$ SR17

\#S $4.5 .1 / 36 / 3$ :

$04.01 .07 /$ SR0 $404.01 .07 /$ SR05 $04.01 .07 /$ SR11

\#S $4.5 .1 / 36 / 6$ :

$04.01 .07 /$ SR12 $04.01 .07 /$ SR13 $04.01 .07 /$ SR14

\#S $4.5 .1 / 37 / 1$ :

$02.03 .03 /$ SR17

\#S $4.5 .1 / 37 / 2$ :

04.01.07/SR09 04.01.07/SR10 04.01.07/SR11 04.01.07/SR12

$04.01 .07 /$ SR13 $04.01 .07 /$ SR14

\#S $4.5 .1 / 38 / 1$ :

$04.01 .07 /$ SR 12

\#S $4.5 .2 / 38 / 3$ :

$04.02 .01 .01 /$ SR03 $04.02 .01 .01 /$ SR0 $404.02 .01 .01 /$ SR0 5

$04.02 .01 .01 /$ SR13 $04.02 .01 .01 /$ SR14 $04.02 .01 .01 /$ SR19

$04.02 .01 .01 / \mathrm{SR} 20 \quad 04.02 .01 .02 / \mathrm{SR} 03 \quad 04.02 .01 .02 / \mathrm{SR} 04$

$04.02 .01 .02 /$ SR05 $04.02 .02 .01 /$ SR03 $04.02 .02 .01 /$ SR0 4

$04.02 .02 .01 /$ SR0 $504.02 .02 .01 /$ SR13 $04.02 .02 .01 /$ SR14 
$04.02 .02 .01 / \mathrm{SR} 20$

$04.02 .02 .02 /$ SR0 4

$04.02 .03 .01 /$ SR12

$04.02 .03 .01 /$ SR27

$04.02 .03 .01 / \mathrm{SR} 40$

$04.02 .03 .01 /$ SR 47

$04.02 .03 .01 /$ SR 64

$04.02 .03 .01 /$ SR7 4

$04.02 .03 .02 / \mathrm{SR} 03$

$04.02 .04 .01 / \mathrm{SR} 05$

$04.02 .04 .01 / \mathrm{SR} 26$

$04.02 .04 .01 / \mathrm{SR} 42$

$04.02 .04 .01 /$ SR5 7

$04.02 .04 .01 /$ SR70

$04.02 .04 .01 /$ SR7 6

$04.02 .04 .02 /$ SR03

$04.02 .05 .01 /$ SR0 5

$04.02 .05 .01 /$ SR19

$04.02 .05 .01 /$ SR32

$04.02 .05 .01 / \mathrm{SR} 41$

$04.02 .05 .01 /$ SR 55

$04.02 .05 .01 / \mathrm{SR} 62$

$04.02 .05 .01 /$ SR 68

$04.02 .05 .02 / S R 04$

$04.02 .05 .03 / \mathrm{SR} 03$

$04.03 .04 .01 /$ SR 15
$04.02 .02 .01 / \mathrm{SR} 21 \quad 04.02 .02 .02 / \mathrm{SR} 03$

$04.02 .02 .02 /$ SR0 $504.02 .03 .01 /$ SR1 1

$04.02 .03 .01 / \mathrm{SR} 1304.02 .03 .01 / \mathrm{SR} 25$

$04.02 .03 .01 /$ SR34 $04.02 .03 .01 /$ SR35

$04.02 .03 .01 /$ SR4 $104.02 .03 .01 / S R 46$

$04.02 .03 .01 /$ SR52 $04.02 .03 .01 /$ SR5 8

$04.02 .03 .01 /$ SR6 $64.02 .03 .01 / S R 72$

$04.02 .03 .01 /$ SR79 $04.02 .03 .01 /$ SR8 0

$04.02 .03 .02 /$ SR0 $404.02 .03 .02 /$ SR0 5

$04.02 .04 .01 /$ SR0 $604.02 .04 .01 /$ SR19

$04.02 .04 .01 /$ SR32 $04.02 .04 .01 /$ SR37

$04.02 .04 .01 / \mathrm{SR} 4804.02 .04 .01 / \mathrm{SR} 55$

$04.02 .04 .01 /$ SR $6304.02 .04 .01 /$ SR 65

$04.02 .04 .01 /$ SR71 $04.02 .04 .01 /$ SR75

$04.02 .04 .02 /$ SR01 $04.02 .04 .02 /$ SR0 2

$04.02 .05 .01 /$ SR03 $04.02 .05 .01 /$ SR0 4

$04.02 .05 .01 /$ SR0 $704.02 .05 .01 /$ SR1 8

$04.02 .05 .01 / \mathrm{SR} 2604.02 .05 .01 / \mathrm{SR} 27$

$04.02 .05 .01 /$ SR34 $04.02 .05 .01 /$ SR 40

$04.02 .05 .01 /$ SR4 $904.02 .05 .01 /$ SR50

$04.02 .05 .01 /$ SR5 $604.02 .05 .01 /$ SR 61

$04.02 .05 .01 /$ SR66 $04.02 .05 .01 /$ SR 67

$04.02 .05 .01 /$ SR69 $04.02 .05 .02 /$ SR0 3

$04.02 .05 .02 /$ SR0 $504.02 .05 .02 /$ SR0 6

$04.02 .05 .03 /$ SR0 $404.02 .05 .03 /$ SR0 5

$04.03 .01 /$ SR0 7

\#S $4.5 .2 / 38 / 7$ :

$04.02 .01 .02 /$ SR0 $604.02 .01 .02 /$ SR0 $04.02 .02 .02 / S R 06$

$04.02 .02 .02 /$ SR0 $704.02 .03 .02 /$ SR0 $604.02 .03 .02 /$ SR0 7

$04.02 .04 .02 /$ SR0 $404.02 .04 .02 /$ SR0 $5 \quad 04.02 .05 .02 /$ SR0 7

$04.02 .05 .02 /$ SR0 $804.02 .05 .03 /$ SR0 $604.02 .05 .03 /$ SR0 $0404.03 .01 /$ SR0 9

\#S $4.5 .2 / 39 / 2:$

$04.03 .01 /$ SR0 $804.03 .01 /$ SR09

\#S $4.5 .2 / 39 / 3$ :

$04.03 .02 .02 /$ SR1 5

\#S $4.5 .2 / 39 / 5:$

$04.03 .01 /$ SR0 $404.03 .01 /$ SR0 $504.03 .01 /$ SR0 $604.03 .01 /$ SR 10

\#S $4.5 .2 / 39 / 6:$

$04.03 .01 /$ SR01

\#S $4.5 .2 / 39 / 7:$

$04.02 .01 .01 /$ SR0 $604.02 .01 .01 /$ SR0 $704.02 .01 .01 / S R 15$

$04.02 .01 .01 /$ SR1 $604.02 .01 .01 /$ SR21 $04.02 .01 .01 /$ SR2 2

$04.02 .01 .02 /$ SR0 $604.02 .01 .02 /$ SR0 $704.02 .01 .02 /$ SR3 5

$04.02 .01 .02 / S R 4304.02 .01 .02 / S R 44 \quad 04.02 .01 .02 / S R 45$

$04.02 .01 .02 /$ SR $4704.02 .01 .02 / S R 48 \quad 04.02 .01 .02 / S R 49$

$04.02 .02 .01 /$ SR0 $64.02 .02 .01 /$ SR0 $0404.02 .02 .01 /$ SR15

$04.02 .02 .01 /$ SR1 $604.02 .02 .01 /$ SR22 $04.02 .02 .01 /$ SR23

$04.02 .02 .02 /$ SR0 $604.02 .02 .02 /$ SR0 $04.02 .02 .02 /$ SR 35 
$04.02 .02 .02 / S R 43 \quad 04.02 .02 .02 / S R 44 \quad 04.02 .02 .02 / S R 45$

$04.02 .02 .02 /$ SR $4804.02 .02 .02 / S R 4904.02 .02 .02 / S R 50$

$04.02 .03 .01 / S R 2104.02 .03 .01 / S R 2204.02 .03 .01 / S R 28$

$04.02 .03 .01 /$ SR $3704.02 .03 .01 /$ SR $4304.02 .03 .01 / S R 48$

$04.02 .03 .01 /$ SR4 $904.02 .03 .02 /$ SR0 $604.02 .03 .02 /$ SR0 7

$04.02 .03 .02 /$ SR38 $04.02 .03 .02 /$ SR3 $904.02 .03 .02 / S R 40$

$04.02 .03 .02 /$ SR57 $04.02 .03 .02 /$ SR $6104.02 .03 .02 /$ SR 64

$04.02 .03 .02 /$ SR $6504.02 .03 .02 /$ SR6 $604.02 .04 .01 /$ SR 14

$04.02 .04 .01 /$ SR15 $04.02 .04 .01 /$ SR20 $04.02 .04 .01 /$ SR2 9

$04.02 .04 .01 /$ SR34 $04.02 .04 .01 /$ SR3 $804.02 .04 .01 /$ SR3 9

$04.02 .04 .02 /$ SR0 $404.02 .04 .02 /$ SR0 $504.02 .04 .02 / S R 11$

$04.02 .04 .02 /$ SR12 $04.02 .04 .02 /$ SR13 $04.02 .04 .02 /$ SR2 4

$04.02 .04 .02 /$ SR27 $04.02 .04 .02 / S R 2904.02 .04 .02 / S R 30$

$04.02 .04 .02 /$ SR31 $04.02 .05 .01 /$ SR0 $804.02 .05 .01 /$ SR0 9

$04.02 .05 .01 /$ SR10 $04.02 .05 .01 /$ SR13 $04.02 .05 .01 /$ SR1 4

$04.02 .05 .01 /$ SR15 $04.02 .05 .01 /$ SR20 $04.02 .05 .01 /$ SR2 1

$04.02 .05 .01 /$ SR28 $04.02 .05 .01 /$ SR2 $904.02 .05 .01 /$ SR 35

$04.02 .05 .01 /$ SR $4204.02 .05 .01 /$ SR $4304.02 .05 .01 /$ SR5 1

$04.02 .05 .01 /$ SR52 $04.02 .05 .01 /$ SR5 $704.02 .05 .01 /$ SR5 8

$04.02 .05 .02 /$ SR0 $704.02 .05 .02 /$ SR0 $804.02 .05 .02 / S R 35$

$04.02 .05 .02 /$ SR3 $94.02 .05 .02 /$ SR5 $104.02 .05 .02 /$ SR5 2

$04.02 .05 .02 /$ SR53 $04.02 .05 .03 /$ SR0 $604.02 .05 .03 /$ SR0 7

$04.02 .05 .03 /$ SR30 $04.02 .05 .03 /$ SR3 $804.02 .05 .03 /$ SR 46

$04.02 .05 .03 /$ SR $4704.02 .05 .03 /$ SR $48 \quad 04.02 .05 .03 / S R 50$

$04.02 .05 .03 /$ SR51 $04.02 .05 .03 /$ SR52 $04.03 .01 /$ SR0 $804.03 .01 /$ SR0 9

\#S $4.5 .2 / 40 / 1:$

$04.01 / S R 0104.03 .03 / S R 0104.03 .03 / S R 04$

\#S $4.5 .2 / 40 / 3:$

$04.02 .01 .01 /$ SR03 $04.02 .01 .02 /$ SR03 $04.02 .02 .01 /$ SR0 3

$04.02 .02 .02 /$ SR03 $04.02 .03 .01 /$ SR11 $04.02 .05 .03 /$ SR03 $04.03 .01 /$ SR0 4

$04.03 .01 /$ SR0 $704.03 .04 .01 /$ SR15

\#S $4.5 .2 / 41 / 2$ :

$04.02 .04 .01 / \mathrm{SR} 05$

\#S $4.5 .2 / 41 / 3$ :

$04.02 .05 .01 / \mathrm{SR} 03$

\#S $4.5 .2 / 41 / 4:$

$04.02 .05 .01 /$ SR0 4

\#S $4.5 .2 / 42 / 2:$

$04.03 .01 /$ SR0 $804.03 .01 /$ SR0 9

\#S $4.5 .2 / 42 / 3:$

$04.02 .01 .02 /$ SR10 $04.02 .01 .02 /$ SR11 $04.02 .01 .02 / S R 22$

$04.02 .01 .02 /$ SR2 $404.02 .01 .02 / S R 2504.02 .02 .02 / S R 10$

$04.02 .02 .02 /$ SR11 $04.02 .02 .02 /$ SR22 $04.02 .02 .02 / S R 24$

$04.02 .02 .02 /$ SR25 $04.02 .03 .02 /$ SR10 $04.02 .03 .02 /$ SR 11

$04.02 .03 .02 /$ SR22 $04.02 .03 .02 / S R 2404.02 .03 .02 / S R 25$

$04.02 .04 .02 /$ SR09 $04.02 .05 .02 /$ SR11 $04.02 .05 .02 /$ SR12

$04.02 .05 .02 /$ SR23 $04.02 .05 .02 / S R 2504.02 .05 .02 /$ SR 26 
$04.02 .05 .03 /$ SR10 $04.02 .05 .03 /$ SR2 5 $04.02 .05 .04 / \mathrm{SR} 17$ $04.03 .02 .02 /$ SR13
$04.02 .05 .03 /$ SR22 $04.02 .05 .03 / S R 24$ $04.02 .05 .04 /$ SR02 $04.02 .05 .04 /$ SR1 6 $04.03 .02 .01 /$ SR02 $04.03 .02 .02 /$ SR12 $04.03 .02 .02 /$ SR1 4
\#S $4.5 .2 / 43 / 2:$

$04.02 .01 .02 /$ SR 10

$04.02 .02 .02 /$ SR 11

$04.02 .05 .03 /$ SR10
$04.02 .01 .02 / \mathrm{SR} 11$

$04.02 .03 .02 /$ SR10

$04.03 .02 .01 / \mathrm{SR} 02$

\#S $4.5 .2 / 43 / 3:$

$04.02 .01 .01 /$ SR0 7

$04.02 .01 .02 /$ SR 48

$04.02 .02 .01 / S R 23$

$04.02 .02 .02 /$ SR 50

$04.02 .03 .02 /$ SR0 7

$04.02 .01 .01 / S R 22$

$04.02 .01 .02 /$ SR 49

$04.02 .02 .02 /$ SR0 7

$04.02 .03 .01 /$ SR22

$04.02 .03 .02 /$ SR6 6

$04.02 .04 .01 /$ SR79

$04.02 .04 .01 /$ SR3 9

$04.02 .04 .02 /$ SR31

$04.02 .05 .01 /$ SR5 8

$04.02 .05 .03 /$ SR0 7

$04.02 .05 .01 / S R 21$

$04.02 .05 .02 /$ SR0 8

$04.02 .05 .03 /$ SR5 1

$04.02 .02 .02 /$ SR 10

$04.02 .05 .02 /$ SR 11

\#S $4.5 .3 / 43 / 4:$

$04.02 .01 .02 /$ SR0 6

$04.02 .01 .02 / S R 43$

$04.02 .01 .02 /$ SR 47

$04.02 .01 .02 /$ SR0 7

$04.02 .01 .02 / S R 44$

$04.02 .01 .02 / S R 48$

$04.02 .01 .02 /$ SR0 7

$04.02 .02 .01 /$ SR0 7

$04.02 .02 .02 /$ SR 49

$04.02 .03 .01 /$ SR 49

$04.02 .04 .01 /$ SR15

$04.02 .04 .02 /$ SR0 5

$04.02 .05 .01 /$ SR29

$04.02 .05 .02 /$ SR53

$04.02 .05 .03 /$ SR5 2

\#S $4.5 .3 / 43 / 5:$

$04.02 .01 .01 /$ SR0 $604.02 .01 .01 /$ SR0 $804.02 .01 .01 /$ SR0 9

$04.02 .01 .01 /$ SR10 $04.02 .01 .02 /$ SR30 $04.02 .01 .02 /$ SR 34

$04.02 .01 .02 /$ SR $3604.02 .01 .02 /$ SR3 $04.02 .01 .02 /$ SR3 8

\#S $4.5 .3 / 43 / 6:$

$04.02 .01 .01 /$ SR15 $04.02 .01 .01 /$ SR16 $04.02 .01 .02 /$ SR 44

$04.02 .01 .02 /$ SR 45

\#S $4.5 .4 / 43 / 7:$

$04.02 .02 .02 /$ SR0 $604.02 .02 .02 /$ SR0 $704.02 .02 .02 /$ SR 35

$04.02 .02 .02 / S R 4304.02 .02 .02 / S R 44 \quad 04.02 .02 .02 / S R 45$

$04.02 .02 .02 /$ SR $4804.02 .02 .02 /$ SR $4904.02 .02 .02 /$ SR 50

\#S $4.5 .4 / 44 / 2$ :

$04.02 .02 .01 /$ SR0 $604.02 .02 .01 /$ SR0 $804.02 .02 .01 /$ SR0 9

$04.02 .02 .01 /$ SR10 $04.02 .02 .02 /$ SR30 $04.02 .02 .02 /$ SR3 4

$04.02 .02 .02 /$ SR $3604.02 .02 .02 /$ SR $37 \quad 04.02 .02 .02 /$ SR 38

\#S $4.5 .4 / 44 / 3$ :

$04.02 .02 .01 /$ SR15 $04.02 .02 .01 /$ SR16 $04.02 .02 .01 /$ SR1 7

$04.02 .02 .02 / S R 4404.02 .02 .02 / S R 45 \quad 04.02 .02 .02 / S R 46$

\#S $4.5 .4 / 44 / 4:$

$06.01 .02 /$ SR 59

\#S $4.5 .5 / 44 / 6$ :

$04.02 .03 .01 /$ SR0 $104.02 .03 .01 /$ SR02 
\#S $4.5 .5 / 44 / 7$ :

$04.02 .03 .01 /$ SR01 $04.02 .03 .01 /$ SR02

\#S $4.5 .5 / 44 / 9:$

$04.02 .03 .01 /$ SR60 $04.02 .03 .03 /$ SR0 $904.02 .04 .01 /$ SR 50

\#S $4.5 .5 / 44 / 10:$

$04.02 .03 .01 /$ SR21

$04.02 .03 .01 /$ SR 37

$04.02 .03 .01 /$ SR 49

$04.02 .03 .02 /$ SR3 8

$04.02 .03 .02 /$ SR 57

$04.02 .03 .02 /$ SR 65

$04.02 .04 .01 /$ SR15

$04.02 .04 .01 / \mathrm{SR} 34$

$04.02 .04 .02 /$ SR0 4

$04.02 .04 .02 / S R 12$

$04.02 .04 .02 / \mathrm{SR} 27$

$04.02 .03 .01 / \mathrm{SR} 22$

$04.02 .03 .01 / \mathrm{SR} 28$

$04.02 .03 .01 / S R 4304.02 .03 .01 / S R 48$

$04.02 .03 .02 /$ SR0 $604.02 .03 .02 /$ SR0 7

$04.02 .03 .02 /$ SR3 $904.02 .03 .02 /$ SR 40

$04.02 .03 .02 /$ SR61 $04.02 .03 .02 /$ SR64

$04.02 .03 .02 /$ SR6 $604.02 .04 .01 /$ SR14

$04.02 .04 .01 /$ SR20 $04.02 .04 .01 /$ SR2 9

$04.02 .04 .01 /$ SR38 $04.02 .04 .01 /$ SR39

$04.02 .04 .02 /$ SR0 $504.02 .04 .02 /$ SR11

$04.02 .04 .02 /$ SR13 $04.02 .04 .02 /$ SR2 4

$04.02 .04 .02 /$ SR3 1

$04.02 .04 .02 / S R 30$

\#S $4.5 .5 / 45 / 2$ :

$04.02 .03 .01 /$ SR36 $04.02 .03 .01 /$ SR5 $904.02 .03 .01 /$ SR 60

$04.02 .03 .01 /$ SR61 $04.02 .03 .01 /$ SR65 $04.02 .03 .01 /$ SR 67

$04.02 .03 .01 /$ SR68 $04.02 .03 .01 /$ SR69 $04.02 .03 .01 / S R 81$

$04.02 .03 .02 /$ SR58 $04.02 .03 .03 /$ SR01 $04.02 .03 .03 /$ SR0 6

$04.02 .03 .03 /$ SR0 8

$04.02 .04 .01 / S R 27$

$04.02 .04 .01 /$ SR52

$04.02 .04 .01 /$ SR5 9

$04.02 .03 .03 /$ SR0 $904.02 .03 .03 /$ SRI0

$04.02 .04 .01 /$ SR $4904.02 .04 .01 /$ SR 50

$04.02 .04 .01 / S R 5604.02 .04 .01 / S R 58$

$04.02 .04 .02 / \mathrm{SR} 25$

$04.02 .04 .01 /$ SR60 $04.02 .04 .01 /$ SR72

\#S $4.5 .5 / 45 / 3:$

$04.02 .03 .01 /$ SR42 $04.02 .03 .02 / S R 6204.02 .03 .03 / S R 07$

$04.02 .04 .01 /$ SR33 $04.02 .04 .02 /$ SR28

\#S $4.5 .5 / 45 / 4:$

$04.02 .03 .01 / \mathrm{SR} 36$

$04.02 .03 .03 /$ SR0 6

$04.02 .03 .03 / \mathrm{SR} 16$

$04.02 .04 .02 /$ SR 25

$04.02 .03 .01 /$ SR75 $04.02 .03 .02 /$ SR5 8

$04.02 .03 .03 /$ SR11 $04.02 .03 .03 /$ SR15

$04.02 .04 .01 /$ SR27 $04.02 .04 .01 /$ SR6 6

\#S $4.5 .5 / 45 / 5$ :

$04.02 .03 .01 /$ SR81 $04.02 .04 .01 /$ SR72

\#S $4.5 .5 / 45 / 6$ :

$04.02 .03 .01 /$ SR73 $04.02 .03 .01 /$ SR75 $04.02 .03 .03 /$ SR 11

$04.02 .04 .01 /$ SR64 $04.02 .04 .01 /$ SR 66

\#S $4.5 .5 / 46 / 1:$

$04.02 .03 .01 /$ SR59 $04.02 .03 .01 /$ SR73 $04.02 .03 .01 / S R 75$

$04.02 .03 .03 /$ SR0 $804.02 .03 .03 /$ SR11 $04.02 .04 .01 / S R 49$

$04.02 .04 .01 /$ SR $6404.02 .04 .01 /$ SR 66 
\#S $4.5 .5 / 46 / 3$ :

$04.02 .03 .01 / \mathrm{SR} 7604.02 .03 .03 / \mathrm{SR} 1204.02 .04 .01 / \mathrm{SR} 67$

\#S $4.5 .5 / 47 / 1$ :

$04.02 .03 .01 /$ SR76 $04.02 .03 .03 /$ SR12 $04.02 .04 .01 /$ SR 67

\#S $4.5 .5 / 47 / 2$ :

$04.02 .03 .01 /$ SR62 $04.02 .03 .01 /$ SR70 $04.02 .03 .01 /$ SR77

$04.02 .03 .01 /$ SR82 $04.02 .04 .01 /$ SR53 $04.02 .04 .01 /$ SR 61

$04.02 .04 .01 /$ SR68 $04.02 .04 .01 /$ SR73

\#S $4.5 .5 / 47 / 4$ :

$04.01 .03 /$ SR0 $84.02 .03 .01 / S R 1404.02 .03 .01 / S R 1504.02 .03 .01 / S R 20$

$04.02 .03 .01 / S R 2104.02 .03 .02 / S R 3004.02 .03 .02 / S R 31$

$04.02 .03 .02 / S R 4004.02 .03 .02 / S R 4104.02 .03 .02 / S R 42$

$04.02 .03 .02 / S R 4604.02 .04 .01 / S R 0704.02 .04 .01 / S R 08$

$04.02 .04 .01 /$ SR13 $04.02 .04 .01 /$ SR14 $04.02 .04 .02 / S R 13$

$04.02 .04 .02 / S R 1404.02 .04 .02 / S R 15 \quad 04.02 .04 .02 / S R 19$

\#S $4.5 .5 / 47 / 5$ :

$04.02 .03 .01 / \mathrm{SR} 2604.02 .03 .01 / \mathrm{SR} 2904.02 .03 .01 / \mathrm{SR} 30$

$04.02 .03 .01 /$ SR3 $04.02 .03 .01 /$ SR61 $04.02 .03 .02 /$ SR34

$04.02 .03 .02 / S R 4804.02 .03 .02 / S R 4904.02 .03 .02 / S R 59$

$04.02 .04 .01 /$ SR18 $04.02 .04 .01 / S R 2104.02 .04 .01 / S R 22$

$04.02 .04 .01 /$ SR2 $904.02 .04 .01 /$ SR52 $04.02 .04 .02 /$ SR10

$04.02 .04 .02 / S R 2104.02 .04 .02 / S R 2204.02 .04 .02 / S R 2606.01 .02 / S R 60$

\#S $4.5 .5 / 48 / 1$ :

$04.02 .03 .01 /$ SR31 $04.02 .03 .02 /$ SR50 $04.02 .03 .03 /$ SR1 4

$04.02 .04 .01 /$ SR23 $04.02 .04 .02 / S R 23$

\#S $4.5 .5 / 48 / 2$ :

$06.01 .02 /$ SR57

\#S $4.5 .5 / 48 / 3$ :

$04.02 .03 .01 /$ SR0 $304.02 .03 .01 /$ SR0 $404.02 .03 .01 /$ SR0 6

$04.02 .03 .01 /$ SR0 $704.02 .03 .01 /$ SR2 $904.02 .03 .01 /$ SR30

$04.02 .03 .02 / S R 4804.02 .03 .02 / S R 49 \quad 04.02 .04 .02 / S R 21$

$04.02 .04 .02 / S R 22$

\#S $4.5 .5 / 48 / 4$ :

$04.02 .03 .01 /$ SR05 $04.02 .03 .01 / S R 08$

\#S $4.5 .5 / 48 / 5$ :

$04.02 .03 .01 /$ SR14 $04.02 .03 .01 /$ SR15 $04.02 .03 .01 /$ SR1 8

$04.02 .03 .01 /$ SR19 $04.02 .03 .01 /$ SR2 $804.02 .03 .02 / S R 31$

$04.02 .03 .02 /$ SR $3604.02 .03 .02 / S R 4104.02 .03 .02 / S R 42$

$04.02 .03 .02 /$ SR $4504.02 .03 .02 /$ SR $4704.02 .03 .03 /$ SR0 4

$04.02 .04 .01 /$ SR0 $704.02 .04 .01 /$ SR0 $804.02 .04 .01 /$ SR1 1

$04.02 .04 .01 /$ SR12 $04.02 .04 .01 /$ SR20 $04.02 .04 .02 /$ SR1 4

$04.02 .04 .02 / S R 1504.02 .04 .02 / S R 18 \quad 04.02 .04 .02 / S R 20$

\#S $4.5 .5 / 48 / 6$ :

$04.02 .03 .01 /$ SR16 $04.02 .03 .01 /$ SR17 $04.02 .03 .01 / S R 21$ 
$04.02 .03 .02 / S R 40 \quad 04.02 .03 .02 / S R 43 \quad 04.02 .03 .02 / S R 44$ $04.02 .03 .03 /$ SRO 2 $04.02 .04 .01 /$ SR0 9 $04.02 .04 .02 /$ SR 13 $04.02 .03 .03 /$ SR03 $04.02 .04 .01 /$ SR10 $04.02 .04 .01 /$ SR1 4 $04.02 .03 .03 /$ SR0 4

\#S $4.5 .5 / 48 / 7$ :

$04.02 .03 .01 /$ SR03

$04.02 .03 .01 /$ SR0 7

$04.02 .03 .02 /$ SR 43

$04.02 .04 .01 /$ SR0 9

$04.02 .03 .01 / \mathrm{SR} 0404.02 .03 .01 / \mathrm{SR} 06$

$04.02 .03 .01 /$ SR1 $604.02 .03 .01 /$ SR59

$04.02 .04 .02 /$ SR1 6

$04.02 .03 .03 /$ SR02 $04.02 .03 .03 /$ SR0 8

\#S $4.5 .5 / 49 / 2$ :

$04.02 .03 .01 /$ SR3 $704.02 .03 .01 /$ SR61 $04.02 .03 .02 /$ SR5 9

$04.02 .04 .01 /$ SR2 $904.02 .04 .01 /$ SR52 $04.02 .04 .02 /$ SR2 6

\#S $4.5 .5 / 49 / 4:$

$04.02 .03 .01 /$ SR3 $704.02 .03 .02 /$ SR59 $04.02 .04 .01 /$ SR2 9 $04.02 .04 .02 /$ SR2 6

\#S $4.5 .5 / 49 / 5:$

$04.02 .03 .01 /$ SR03 $04.02 .03 .01 /$ SR0 $404.02 .03 .01 /$ SR0 6

$04.02 .03 .01 /$ SR0 $704.02 .03 .01 /$ SR29 $04.02 .03 .01 /$ SR 30

$04.02 .03 .01 /$ SR $3104.02 .03 .02 / S R 48 \quad 04.02 .03 .02 / S R 49$

$04.02 .03 .02 /$ SR50 $04.02 .03 .02 /$ SR59 $04.02 .03 .03 /$ SR14

$04.02 .04 .01 /$ SR21 $04.02 .04 .01 /$ SR22 $04.02 .04 .01 /$ SR23

$04.02 .04 .01 /$ SR51 $04.02 .04 .02 /$ SR21 $04.02 .04 .02 /$ SR2 2

$04.02 .04 .02 /$ SR23 $04.02 .04 .02 / S R 26$

\#S $4.5 .5 / 51 / 1$ :

$04.02 .03 .03 /$ SR0 7

\#S $4.5 .5 / 52 / 1$ :

$04.02 .03 .03 /$ SR0 7

\#S $4.5 .6 / 55 / 1:$

$04.01 .04 /$ SR0 $04.01 .04 /$ SR09 $04.02 .04 .01 /$ SR01 $04.02 .04 .01 /$ SR02 $04.02 .04 .01 /$ SR28 $04.02 .04 .01 /$ SR5 1

\#S $4.5 .6 / 55 / 3:$

$04.02 .04 .01 / \mathrm{SR} 1404.02 .04 .01 / \mathrm{SR} 15 \quad 04.02 .04 .01 / \mathrm{SR} 20$

$04.02 .04 .01 /$ SR21 $04.02 .04 .01 /$ SR22 $04.02 .04 .01 /$ SR23

$04.02 .04 .01 /$ SR27 $04.02 .04 .01 /$ SR29 $04.02 .04 .01 /$ SR33

$04.02 .04 .01 /$ SR3 $404.02 .04 .01 /$ SR38 $04.02 .04 .01 /$ SR3 9

\#S $4.5 .6 / 55 / 4:$

$04.02 .04 .01 /$ SR $4904.02 .04 .01 /$ SR50 $04.02 .04 .01 /$ SR5 1

$04.02 .04 .01 /$ SR52 $04.02 .04 .01 /$ SR5 $604.02 .04 .01 /$ SR5 8

$04.02 .04 .01 /$ SR5 $904.02 .04 .01 /$ SR60 $04.02 .04 .01 /$ SR 64

$04.02 .04 .01 /$ SR $6604.02 .04 .01 /$ SR $6704.02 .04 .01 /$ SR 68

$04.02 .04 .01 /$ SR72 $04.02 .04 .01 /$ SR73

\#S $4.5 .6 / 55 / 5$ :

$04.02 .04 .01 /$ SR78 $04.02 .04 .01 /$ SR80 $04.02 .04 .01 /$ SR 81 
$04.02 .04 .01 /$ SR8 2

\#S $4.5 .6 / 55 / 6:$

$06.01 .02 /$ SR61 $06.01 .02 /$ SR 62

\#S $4.5 .7 / 55 / 7$ :

$04.02 .03 .03 /$ SR01 $04.02 .03 .03 /$ SR0 $504.02 .03 .03 /$ SR13

$04.02 .03 .03 /$ SR15 $04.02 .03 .03 /$ SR16

\#S $4.5 .7 / 56 / 1$ :

$04.02 .03 .03 /$ SR13

\#S $4.5 .7 / 56 / 2$ :

$04.02 .03 .03 /$ SR15 $04.02 .03 .03 /$ SR1 6

\#S $4.5 .7 / 56 / 3:$

$04.02 .03 .03 /$ SR17 $04.02 .03 .03 /$ SR1 8

\#S $4.5 .7 / 56 / 4:$

$04.02 .03 .03 /$ SR 17

\#S $4.5 .7 / 57 / 1$ :

$04.02 .03 .03 /$ SR0 $704.02 .03 .03 /$ SR17 $04.02 .03 .03 /$ SR 18

\#S $4.5 .8 / 58 / 2:$

$04.02 .05 .02 /$ SR0 $704.02 .05 .02 /$ SR0 $804.02 .05 .02 / S R 35$

$04.02 .05 .02 /$ SR39 $04.02 .05 .02 /$ SR51 $04.02 .05 .02 /$ SR5 2

$04.02 .05 .02 / \mathrm{SR} 53$

\#S $4.5 .8 / 58 / 3$ :

$04.02 .05 .01 /$ SR0 $64.02 .05 .01 /$ SR0 $804.02 .05 .01 / S R 10$

$04.02 .05 .01 /$ SR11 $04.02 .05 .01 /$ SR12 $04.02 .05 .01 /$ SR13

$04.02 .05 .01 /$ SR14 $04.02 .05 .01 /$ SR15 $04.02 .05 .01 /$ SR2 0

$04.02 .05 .01 /$ SR2 $24.02 .05 .01 /$ SR23 $04.02 .05 .02 /$ SR3 1

$04.02 .05 .02 /$ SR3 6

\#S $4.5 .8 / 58 / 4:$

$06.01 .02 /$ SR63

\#S $4.5 .8 / 59 / 1$ :

$04.02 .05 .01 /$ SR71 $04.02 .05 .01 /$ SR72 $04.02 .05 .01 / S R 73$

$04.02 .05 .01 /$ SR75 $04.02 .05 .01 /$ SR76

\#S $4.5 .8 / 60 / 1$ :

$04.02 .05 .01 /$ SR71 $04.02 .05 .01 /$ SR74

\#S $4.5 .8 / 60 / 2$ :

$04.02 .05 .01 /$ SR71 $04.02 .05 .01 /$ SR75 $04.02 .05 .01 /$ SR7 6

$04.02 .05 .01 /$ SR77 $04.02 .05 .01 /$ SR78 $04.02 .05 .01 /$ SR79

\#S $4.5 .8 / 61 / 2$ :

$04.02 .05 .02 /$ SR $4704.02 .05 .02 / S R 48$

\#S $4.5 .8 / 61 / 3$ : 
$04.02 .05 .01 / S R 20 \quad 04.02 .05 .01 / S R 22$

\#S $4.5 .8 / 62 / 1$ :

$04.02 .05 .01 /$ SR23 $04.02 .05 .02 / S R 49$

\#S $4.5 .8 / 62 / 2$ :

$04.02 .05 .02 /$ SR32

\#S $4.5 .9 / 62 / 4$ :

$04.02 .05 .03 /$ SR 11

\#S $4.5 .9 / 62 / 5$ :

$04.02 .05 .01 /$ SR33 $04.02 .05 .01 /$ SR35 $04.02 .05 .01 /$ SR3 6

$04.02 .05 .03 /$ SR0 $604.02 .05 .03 /$ SR0 $04.02 .05 .03 /$ SR2 9

$04.02 .05 .03 /$ SR30 $04.02 .05 .03 /$ SR3 $104.02 .05 .03 /$ SR3 8

$04.02 .05 .03 /$ SR4 $604.02 .05 .03 /$ SR $4704.02 .05 .03 /$ SR 48

$04.02 .05 .03 /$ SR50 $04.02 .05 .03 /$ SR51 $04.02 .05 .03 /$ SR5 2

\#S $4.5 .9 / 62 / 6$ :

$04.02 .05 .01 /$ SR37 $06.01 .02 /$ SR64

\#S $4.5 .9 / 62 / 7$ :

$04.02 .05 .01 /$ SR $4204.02 .05 .01 / S R 43 \quad 04.02 .05 .01 / S R 44$

$04.02 .05 .01 /$ SR4 $504.02 .05 .01 /$ SR4 $604.02 .05 .03 /$ SR33

$04.02 .05 .03 /$ SR37 $04.02 .05 .03 /$ SR39 $04.02 .05 .03 /$ SR 40

$04.02 .05 .03 /$ SR 41

\#S $4.5 .9 / 62 / 8$ :

$04.02 .05 .01 /$ SR51 $04.02 .05 .01 /$ SR52 $04.02 .05 .03 /$ SR 47

$04.02 .05 .03 / \mathrm{SR} 48$

\#S $4.5 .9 / 62 / 9$ :

$04.02 .05 .01 /$ SR37

\#S $4.5 .9 / 62 / 11$ :

$06.01 .02 / \mathrm{SR} 64$

\#S $4.5 .12 / 63 / 4$ :

$04.02 .01 .01 /$ SR21 $04.02 .01 .02 / S R 48 \quad 04.02 .01 .02 / S R 49$

$04.02 .02 .01 /$ SR22 $04.02 .02 .02 /$ SR $4904.02 .02 .02 /$ SR5 0

$04.02 .03 .01 /$ SR $4804.02 .03 .02 /$ SR $6504.02 .03 .02 /$ SR 66

$04.02 .04 .01 /$ SR38 $04.02 .04 .02 /$ SR30 $04.02 .04 .02 /$ SR31

$04.02 .05 .01 /$ SR28 $04.02 .05 .01 /$ SR5 $04.02 .05 .02 /$ SR5 2

$04.02 .05 .02 /$ SR53 $04.02 .05 .03 /$ SR5 $104.02 .05 .03 /$ SR52

\#S $4.5 .12 / 63 / 5$ :

$04.03 .02 .01 /$ SR0 $804.03 .02 .02 / S R 1004.03 .02 .02 / S R 11$

$04.03 .02 .02 /$ SR12 $04.03 .02 .02 /$ SR30 $04.03 .02 .02 /$ SR31

\#S $4.5 .12 / 63 / 6$ :

$04.03 .02 .02 /$ SR 20

\#S $4.5 .12 / 63 / 7$ :

04.03.02.01/SR01 $04.03 .02 .01 /$ SR02 $04.03 .02 .01 / S R 04$ 
04.03.02.01/SR05 $04.03 .02 .02 / S R 27 \quad 04.03 .02 .02 / S R 32$

\#S $4.5 .12 / 63 / 8$ :

$04.03 .02 .01 /$ SR02 $04.03 .02 .01 /$ SR0 $504.03 .02 .01 / S R 09$

$04.03 .02 .01 /$ SR10 $04.03 .02 .02 / S R 2104.03 .02 .02 / S R 27$

$04.03 .02 .02 /$ SR32

\#S $4.5 .12 / 64 / 1$ :

$04.03 .02 .02 /$ SR16 $04.03 .02 .02 /$ SRI7 $04.03 .02 .02 /$ SR18

$04.03 .02 .02 /$ SR19 $04.03 .02 .02 / S R 2204.03 .02 .02 / S R 23$

$04.03 .02 .02 /$ SR2 $404.03 .02 .02 /$ SR2 $504.03 .02 .02 /$ SR2 6

$04.03 .02 .02 /$ SR2 $84.03 .02 .02 /$ SR2 9

\#S $4.5 .12 / 64 / 2$ :

$04.03 .02 .02 / \mathrm{SR} 33$

\#S $4.5 .12 / 64 / 3$ :

$04.03 .02 .02 / S R 0404.03 .02 .02 / S R 0504.03 .02 .02 / S R 06$

\#S $4.5 .13 / 64 / 4$ :

$04.01 /$ SR0 1

\#S $4.5 .15 / 64 / 7$ :

$04.03 .04 .01 / \mathrm{SR} 0104.03 .04 .01 / \mathrm{SR} 0404.03 .04 .01 / \mathrm{SR} 12$

$04.03 .04 .01 /$ SR13 $04.03 .04 .01 /$ SR1 6

\#S $4.5 .15 / 64 / 8$ :

$04.03 .04 .02 /$ SR0 $104.03 .04 .02 / S R 04 \quad 04.03 .04 .02 / S R 05$

$04.03 .04 .02 /$ SR0 8

\#S $4.5 .15 / 65 / 2$ :

$04.03 .04 .01 / \mathrm{SR} 1204.03 .04 .01 / \mathrm{SR} 1304.03 .04 .01 / \mathrm{SR} 16$

\#S $4.5 .15 / 65 / 4$ :

$04.03 .04 .01 /$ SR07 $04.03 .04 .01 / S R 0804.03 .04 .01 / S R 09$

$04.03 .04 .02 /$ SR10 $04.03 .04 .02 /$ SR11

\#S $4.5 .16 / 65 / 5$ :

$04.01 .07 /$ SR14 $04.02 .03 .02 /$ SR31

\#S $4.6 .1 / 67 / 2$ :

$04.01 /$ SR02 $05.01 .01 /$ SR0 $805.01 .02 /$ SR0 1

\#S $4.6 .1 / 67 / 3$ :

$04.01 /$ SR02

\#S $4.6 .1 / 68 / 1$ :

$05.01 .02 / \mathrm{SR} 02$

\#S $4.6 .2 / 68 / 7$ :

$05.01 .01 /$ SR0 $605.01 .01 /$ SR0 $805.01 .02 /$ SR01 $05.01 .02 /$ SR0 6

\#S $4.6 .3 / 69 / 2$ :

$04.03 .03 /$ SR0 $6 \quad 04.03 .03 /$ SR0 $7 \quad 05.02 /$ SR0 $6 \quad 05.02 /$ SR0 7 
\#S $4.6 .3 / 69 / 3:$

$05.02 /$ SR1 $905.02 / S R 2005.02 / S R 21$

\#S $4.6 .3 / 70 / 1:$

$05.02 / \mathrm{SR} 03$

\#S $4.6 .3 / 70 / 2:$

$05.02 / S R 1105.02 / S R 12 \quad 05.02 / S R 13 \quad 05.02 / S R 14 \quad 05.02 / S R 15$

\#S $4.6 .3 / 70 / 3:$

$05.02 / \mathrm{SR} 04$

\#S $4.6 .3 / 70 / 4:$

$05.02 / S R 01$

\#S $4.6 .3 / 70 / 5$ :

$05.02 / \operatorname{SR} 22$

\#S $4.6 .3 / 70 / 6$ :

$05.02 / S R 29$

\#S $4.6 .3 / 70 / 7:$

$05.02 /$ SR30 $05.02 /$ SR31 $05.02 /$ SR32

\#S $4.6 .3 / 70 / 9:$

$04.03 .03 /$ SR0 $604.03 .03 / S R 07 \quad 05.02 / S R 16 \quad 05.02 / S R 1705.02 / S R 23$

$05.02 / S R 2405.02 / S R 2505.02 / S R 26 \quad 05.02 / S R 2705.02 / S R 28$

\#S $4.6 .3 / 71 / 1:$

$04.03 .03 /$ SR0 $604.03 .03 /$ SR0 $005.02 / S R 02 \quad 05.02 / S R 23 \quad 05.02 / S R 25$

$05.02 / S R 2605.02 / S R 2705.02 / S R 28$

\#S $4.6 .3 / 72 / 1:$

$05.02 / S R 1605.02 / S R 1705.02 / S R 22 \quad 05.02 / S R 2305.02 / S R 2405.02 / S R 25$

$05.02 / S R 2605.02 / S R 2705.02 / S R 28 \quad 05.02 / S R 2905.02 / S R 3105.02 / S R 32$

\#S $4.6 .3 / 73 / 1:$

$05.02 / S R 1605.02 / S R 1705.02 / S R 22 \quad 05.02 / S R 23 \quad 05.02 / S R 2405.02 / S R 25$

$05.02 / S R 2605.02 / S R 2705.02 / S R 28 \quad 05.02 / S R 2905.02 / S R 3105.02 / S R 32$

\#S $4.6 .3 / 74 / 1:$

$05.02 /$ SR19

\#S $4.6 .3 / 75 / 1:$

$05.02 /$ SR19

\#S $4.6 .3 / 75 / 3:$

$05.02 /$ SR23

\#S $4.6 .6 / 76 / 3:$

$04.03 .03 /$ SR01 $04.03 .03 /$ SR0 $404.03 .03 /$ SR09 $04.03 .03 /$ SR10

\#S $4.6 .6 / 76 / 4:$ 
$04.03 .04 .02 /$ SRO9

\#S $4.7 .2 / 77 / 3:$

04.01/SR01 06.01.01/SR01 06.01.01/SR02 06.01.02/SR16

$06.01 .02 /$ SR17 $06.02 .01 /$ SR01 $06.02 .01 /$ SR0 2

\#S $4.7 .2 / 77 / 4:$

$06.01 .02 /$ SR1 9

\#S $4.7 .2 / 77 / 6$ :

$06.01 .02 / S R 20$

\#S $4.7 .2 / 77 / 7:$

$06.01 .02 /$ SR01 $06.01 .02 /$ SR0 $406.01 .02 /$ SR0 $706.01 .02 /$ SR0 8

\#S $4.7 .2 / 78 / 2$ :

$06.01 .02 /$ SR09 $06.01 .02 /$ SR12 $06.01 .02 /$ SR15

\#S $4.7 .2 / 78 / 3:$

$06.01 .02 / S R 57$

\#S $4.7 .2 / 78 / 4:$

06.01.02/SR23 06.01.02/SR26 06.01.02/SR40 06.01.02/SR41 $06.01 .02 / S R 43$

\#S $4.7 .2 / 78 / 5:$

$06.01 .02 / S R 42$

\#S $4.7 .2 / 78 / 6:$

$06.01 .02 / S R 41$

\#S $4.7 .2 / 78 / 7:$

$06.01 .02 / S R 43$

\#S $4.7 .2 / 79 / 1:$

$06.01 .02 / \mathrm{SR} 3606.01 .02 / \mathrm{SR} 4406.01 .02 / \mathrm{SR} 45$

\#S $4.7 .2 / 79 / 2:$

$06.01 .02 /$ SR32 $06.01 .02 /$ SR5 6

\#S $4.7 .2 / 79 / 3:$

$06.01 .02 /$ SR29 $06.01 .02 /$ SR52 $06.01 .02 /$ SR53 $06.01 .02 /$ SR5 8

\#S $4.7 .3 / 79 / 4:$

06.01.01/SR03 06.01.01/SR04 06.01.01/SR05 $06.01 .01 /$ SR0 6

06.01.01/SR07 06.01.01/SR08 06.01.01/SR09 $06.01 .01 /$ SR10

$06.01 .01 /$ SR11 $06.01 .01 /$ SR12 $06.01 .01 /$ SR13 $06.01 .01 /$ SR14

$06.01 .01 /$ SR15 06.01.01/SR16 $06.01 .01 /$ SR17 $06.01 .01 /$ SR18

\#S $4.7 .3 / 80 / 1$ :

$02.03 .03 /$ SR10 $04.01 /$ SR01

\#S $4.7 .4 / 82 / 1:$

$06.02 .01 / S R 01$ 
\#S $4.7 .4 / 83 / 3:$

$06.02 .02 /$ SR0 8

\#S $4.7 .4 / 83 / 4:$

$06.02 .02 / \mathrm{SR} 07$

\#S $4.7 .4 / 83 / 6:$

$06.02 .02 /$ SR09 $06.02 .02 /$ SR10 $06.02 .02 /$ SR11 $06.02 .02 /$ SR1 4 $06.02 .02 /$ SR15

\#S $4.7 .4 / 83 / 7$ :

$06.02 .02 /$ SR1 6

\#S $4.7 .4 / 84 / 1:$

$06.02 .02 / \mathrm{SR} 23$

\#S $4.7 .4 / 84 / 2$ :

$06.02 .02 / \mathrm{SR} 25 \quad 06.02 .02 / \mathrm{SR} 26 \quad 06.02 .02 / \mathrm{SR} 27$

\#S $4.7 .4 / 84 / 3$ :

$06.02 .02 /$ SR2 9

\#S $4.7 .4 / 84 / 4:$

$06.02 .02 /$ SR28

\#S $4.7 .4 / 84 / 5:$

$06.02 .02 /$ SR19 $06.02 .02 /$ SR 20

\#S $4.7 .4 / 84 / 6$ :

$06.02 .02 /$ SR0 1

\#S $4.7 .5 / 84 / 7$ :

$06.02 .01 /$ SR01

\#S $4.7 .5 / 84 / 8$ :

$06.02 .01 /$ SR01

\#S $4.7 .5 / 84 / 9$ :

$06.02 .01 /$ SR03

\#S $4.7 .5 / 84 / 10:$

$06.02 .01 /$ SR0 $409.02 .04 /$ SR01 09.02.04/SR02 $09.02 .04 /$ SR0 3

$09.02 .04 /$ SR0 $409.02 .04 /$ SR05 $09.02 .04 /$ SR0 $609.02 .04 /$ SR0 7

$09.02 .04 /$ SR0 $809.02 .04 /$ SR09 $09.02 .04 /$ SR 10

\#S $4.7 .5 / 85 / 3:$

$06.02 .01 /$ SR0 5

\#S $4.7 .5 / 85 / 4:$

$06.02 .01 /$ SR0 6

\#S $4.7 .5 / 86 / 4:$

$06.02 .01 /$ SR0 7 
\#S $4.7 .5 / 86 / 5:$

$06.02 .01 /$ SR0 9

\#S $4.7 .5 / 86 / 6$ :

$06.02 .01 /$ SR0 8

\#S $4.7 .5 / 86 / 9:$

$06.02 .01 /$ SR10

\#S $4.7 .5 / 87 / 3:$

$09.02 .04 /$ SR0 $109.02 .04 /$ SR02 $09.02 .04 /$ SR0 $309.02 .04 /$ SR0 4

$09.02 .04 /$ SR0 $89.02 .04 /$ SR09 $09.02 .04 /$ SRIO

\#S $4.7 .5 / 89 / 1$ :

$06.02 .01 /$ SR 11

\#S $4.7 .5 / 89 / 2:$

$06.02 .01 /$ SR13

\#S $4.7 .5 / 89 / 3:$

$06.02 .01 /$ SR12

\#S $4.7 .6 / 90 / 5$ :

$04.01 /$ SRO1 $06.03 /$ SR01

\#S $4.7 .6 / 90 / 6:$

$04.02 .04 .01 / \mathrm{SR} 2804.02 .04 .01 / \mathrm{SR} 51 \quad 06.03 / \mathrm{SR} 04$

\#S $4.7 .6 / 91 / 2$ :

$05.02 /$ SR10 06.03/SR01 $06.03 /$ SR0 $86.03 /$ SR11

\#S $4.7 .6 / 91 / 3:$

$06.03 /$ SR0 $606.03 /$ SR07 $06.03 /$ SR09 $06.03 /$ SR10

\#S $4.7 .6 / 91 / 4:$

$06.03 /$ SR14 $06.03 /$ SR15

\#S $4.7 .6 / 91 / 5$ :

$06.03 /$ SR1 $606.03 /$ SR18

\#S $4.7 .6 / 91 / 6$ :

$06.03 / S R 20$

\#S $4.7 .6 / 91 / 7$ :

$06.03 /$ SR17 06.03/SR19

\#S $4.7 .6 / 91 / 9$ :

$04.02 .04 .01 /$ SR28 $04.02 .04 .01 /$ SR5 1

\#S $4.7 .6 / 92 / 4:$

$06.03 /$ SRO 5

\#S $4.10 / 107 / 2$ : 


\section{1/SR01 01/SR02}

\#S $4.10 / 107 / 3$ :

$05.01 .01 /$ SR03 $05.01 .01 /$ SR04 $05.01 .01 /$ SR0 5

\#S $4.10 / 107 / 4:$

$02.02 .01 /$ SR09

\#S $4.10 / 107 / 6:$

$02.02 .01 /$ SR09 $05.01 .01 /$ SR02

\#S $4.10 / 107 / 8:$

$02.01 .01 / \mathrm{SR} 08$

\#S $4.11 / 108 / 1$ :

$04.02 .01 .02 /$ SR14 $04.02 .01 .02 /$ SR15 $04.02 .02 .02 / S R 14$

$04.02 .02 .02 /$ SR15 $04.02 .03 .02 /$ SR14 $04.02 .03 .02 /$ SR15

$04.02 .05 .02 /$ SR15 $04.02 .05 .02 /$ SR1 $604.02 .05 .03 /$ SR1 4

$04.02 .05 .03 /$ SR15 $04.02 .05 .04 /$ SR0 $504.02 .05 .04 / S R 06$

$04.03 .02 .01 /$ SR0 $504.03 .02 .02 /$ SR32 $04.03 .03 /$ SR0 $509.02 .01 /$ SR01

$09.02 .01 /$ SR02 09.02.01/SR03 09.02.01/SR04 $09.02 .10 /$ SR01

$09.02 .10 /$ SR02 09.02.10/SR03 09.02.10/SR04 09.02.10/SR05

$09.02 .11 / \mathrm{SR} 0109.02 .11 / \mathrm{SR} 0209.02 .11 / \mathrm{SR} 03$

\#S 4.11/109/1:

$09.02 .02 /$ SR01 $09.02 .02 /$ SR02 $09.02 .02 /$ SR03 $09.02 .02 /$ SR0 4

$09.02 .02 /$ SR05 $09.02 .02 /$ SR0 $609.02 .02 /$ SR0 $709.02 .02 /$ SR0 8

$09.02 .02 /$ SR09 $09.02 .03 /$ SR01 09.02.03/SR02 $09.02 .03 /$ SR03

$09.02 .03 /$ SR0 $409.02 .03 /$ SR0 5 09.02.03/SR0 $609.02 .03 /$ SR0 7

$09.02 .03 /$ SR0 $809.02 .05 /$ SR01 $09.02 .05 /$ SR02 $09.02 .05 /$ SR03

$09.02 .05 /$ SR0 $409.02 .05 /$ SR0 $509.02 .09 /$ SR0 1

\#S 4.12/109/1:

$09.01 /$ SR0 4

\#S 4.12/109/5:

$09.01 /$ SR11

\#S $4.12 / 109 / 6$ :

09.01.01.01/SR01 09.01.01.01/SR02 09.01.01.01/SR03

09.01.01.01/SR04 09.01.01.01/SR05 09.01.01.01/SR06

09.01.01.01/SR07 09.01.01.02/SR01 09.01.01.02/SR02

$09.01 .01 .02 /$ SR0 3 $09.01 .01 .02 /$ SR0 4 09.01.01.02/SR0 5

09.01.01.02/SR06 09.01.01.02/SR07 09.01.02.01/SR01

09.01.02.01/SR02 09.01.02.01/SR03 09.01.02.01/SR0 4

$09.01 .02 .01 /$ SR0 $509.01 .02 .01 /$ SR0 $609.01 .02 .01 /$ SR0 7

$09.01 .02 .01 /$ SR0 8 $09.01 .02 .01 /$ SR09 $09.01 .02 .01 / S R 10$

09.01.02.01/SR11 09.01.02.01/SR12 09.01.02.02/SR01

$09.01 .02 .02 /$ SR02 $09.01 .02 .02 /$ SR03 $09.01 .02 .02 /$ SR0 4

$09.01 .02 .02 /$ SR05 $09.01 .02 .02 /$ SR0 $609.01 .02 .02 /$ SR0 7

$09.01 .02 .02 /$ SR0 $809.01 .02 .02 /$ SR09 $09.01 .02 .02 /$ SR 10

$09.01 .02 .02 /$ SR11 $09.01 .02 .02 /$ SR12 $09.01 .03 .01 /$ SR01

$09.01 .03 .01 /$ SR02 $09.01 .03 .01 /$ SR03 $09.01 .03 .01 /$ SR0 4

$09.01 .03 .01 /$ SR05 $09.01 .03 .01 /$ SR0 $69.01 .03 .01 /$ SR0 7 
$09.01 .03 .01 / \mathrm{SR} 08$ $09.01 .03 .01 /$ SR11 $09.01 .03 .01 /$ SR14 $09.01 .03 .01 /$ SR17 $09.01 .03 .02 /$ SR03 $09.01 .03 .02 /$ SR0 6 $09.01 .03 .02 /$ SR0 9 $09.01 .03 .02 /$ SR12 $09.01 .03 .02 /$ SR15 $09.01 .04 .01 /$ SR01 $09.01 .04 .01 /$ SR0 4 $09.01 .04 .01 /$ SR0 7 $09.01 .04 .02 / \mathrm{SR} 03$ $09.01 .04 .02 /$ SR0 6 $09.01 .05 .01 /$ SR02 $09.01 .05 .01 /$ SR05 $09.01 .05 .02 /$ SR0 2 $09.01 .05 .02 /$ SR0 5 $09.01 .09 .01 /$ SR02 $09.01 .09 .01 /$ SR0 5 $09.01 .09 .01 /$ SR0 8 $09.01 .09 .02 /$ SR02 $09.01 .09 .02 /$ SR0 5 $09.01 .09 .02 /$ SR0 8 $09.01 .10 .01 /$ SR02 $09.01 .10 .02 /$ SR01 $09.01 .10 .02 /$ SR0 4
$09.01 .03 .01 /$ SR0 9 $09.01 .03 .01 /$ SR12 $09.01 .03 .01 /$ SR15 $09.01 .03 .02 / \mathrm{SR} 01$ $09.01 .03 .02 /$ SR0 4 $09.01 .03 .02 /$ SR0 7 $09.01 .03 .02 /$ SR1 0 $09.01 .03 .02 /$ SR1 3 $09.01 .03 .02 /$ SR1 6 $09.01 .04 .01 /$ SR02 $09.01 .04 .01 /$ SR0 5 $09.01 .04 .02 /$ SR0 1 $09.01 .04 .02 / \mathrm{SR} 04$ $09.01 .04 .02 /$ SR0 7 $09.01 .05 .01 /$ SR0 3 $09.01 .05 .01 /$ SR0 6 $09.01 .05 .02 /$ SR0 3 $09.01 .05 .02 /$ SR0 6 $09.01 .09 .01 /$ SR03 $09.01 .09 .01 /$ SR0 6 $09.01 .09 .01 /$ SR0 9 $09.01 .09 .02 /$ SR0 3 $09.01 .09 .02 /$ SR0 6 $09.01 .09 .02 /$ SR0 9 $09.01 .10 .01 /$ SR0 3 $09.01 .10 .02 / \mathrm{SR} 02$ $09.01 .11 .01 /$ SR0 4
$09.01 .03 .01 /$ SR10 $09.01 .03 .01 /$ SR13 $09.01 .03 .01 /$ SR1 6 $09.01 .03 .02 /$ SR02 $09.01 .03 .02 /$ SR0 5 $09.01 .03 .02 /$ SR0 8 $09.01 .03 .02 /$ SR11 $09.01 .03 .02 /$ SR1 4 $09.01 .03 .02 /$ SR1 7 $09.01 .04 .01 / \mathrm{SR} 03$ $09.01 .04 .01 /$ SR0 6 $09.01 .04 .02 /$ SR02 $09.01 .04 .02 / S R 05$ $09.01 .05 .01 / S R 01$ $09.01 .05 .01 / \mathrm{SR} 04$ $09.01 .05 .02 / S R 01$ $09.01 .05 .02 /$ SR0 4 $09.01 .09 .01 /$ SR0 1 $09.01 .09 .01 /$ SR0 4 $09.01 .09 .01 /$ SR0 7 $09.01 .09 .02 /$ SR01 $09.01 .09 .02 /$ SR0 4 $09.01 .09 .02 /$ SR0 7 $09.01 .10 .01 /$ SR01 $09.01 .10 .01 /$ SR0 4 $09.01 .10 .02 /$ SR0 3 $09.01 .11 .02 /$ SR01

\#S $4.12 / 109 / 7$ :

09.01/SR0 $409.01 /$ SR05 09.01/SR05 09.01/SR0 $609.01 /$ SR0 7

\#S $4.12 / 109 / 8$ : $09.01 /$ SR0 8

\#S $4.12 / 110 / 1$ : $09.01 /$ SR0 9

\#S $4.12 / 110 / 3$ :

09.01/SR01 09.01/SR02 09.01/SR03 09.01/SR10

\#S $4.14 / 113 / 2$ :

$04.02 .03 .02 /$ SR30

\#S $4.14 / 113 / 3$ :

$04.02 .01 .02 / S R 2104.02 .01 .02 / S R 2204.02 .01 .02 / S R 30$

$04.02 .02 .02 / S R 2104.02 .02 .02 / S R 2204.02 .02 .02 / S R 30$

$04.02 .03 .01 /$ SR1 $84.02 .03 .02 / S R 2104.02 .03 .02 / S R 22$

$04.02 .03 .02 /$ SR $4504.02 .03 .03 /$ SR0 $404.02 .04 .01 /$ SR11

$04.02 .04 .01 /$ SR77 $04.02 .04 .02 /$ SR1 $804.02 .05 .02 /$ SR22

$04.02 .05 .02 /$ SR23 $04.02 .05 .02 /$ SR32 $04.02 .05 .03 /$ SR2 1

$04.02 .05 .03 /$ SR22 $04.02 .05 .03 /$ SR33 $04.03 .02 .02 /$ SR19 $06.01 .02 /$ SR36 $06.01 .02 /$ SR37

\#S 4.14/114/2: 
$02.03 .03 / S R 2102.03 .03 / S R 22 \quad 03.01 / S R 02 \quad 04.02 .01 .02 / S R 18$ $04.02 .01 .02 / \mathrm{SR} 2104.02 .01 .02 / \mathrm{SR} 22 \quad 04.02 .01 .02 / \mathrm{SR} 30$ $04.02 .01 .02 /$ SR41 $04.02 .02 .02 /$ SR18 $04.02 .02 .02 /$ SR21 $04.02 .02 .02 / \mathrm{SR} 2204.02 .02 .02 / \mathrm{SR} 30 \quad 04.02 .02 .02 / \mathrm{SR} 41$ $04.02 .03 .01 /$ SR55 $04.02 .03 .02 /$ SR1 8 04.02.03.02/SR21 $04.02 .03 .02 /$ SR22 $04.02 .03 .02 /$ SR31 $04.02 .03 .02 /$ SR32 $04.02 .03 .02 /$ SR33 $04.02 .03 .02 /$ SR53 $04.02 .03 .02 /$ SR5 9 $04.02 .04 .01 /$ SR $4504.02 .04 .02 /$ SR2 $604.02 .05 .02 /$ SR19 $04.02 .05 .02 / S R 22 \quad 04.02 .05 .02 / S R 23 \quad 04.02 .05 .02 / S R 34$ $04.02 .05 .02 / S R 4204.02 .05 .03 /$ SR18 $04.02 .05 .03 / S R 21$ $04.02 .05 .03 / S R 2204.02 .05 .03 / S R 3304.02 .05 .03 / S R 44$ $04.02 .05 .04 /$ SR10 $04.02 .05 .04 /$ SR13 $04.02 .05 .04 /$ SR1 4 $04.03 .02 .02 /$ SR19 $05.01 .02 /$ SR0 $405.03 /$ SR1 $706.01 .02 /$ SR 36 $06.01 .02 / \mathrm{SR} 3706.02 .02 / \mathrm{SR} 05 \quad 06.02 .02 / \mathrm{SR} 13$

\#S $5.4 .2 / 138 / 6:$

$04.02 .03 .01 /$ SR3 $604.02 .03 .01 /$ SR3 $04.02 .03 .02 /$ SR5 8 $04.02 .03 .02 /$ SR5 $904.02 .03 .02 /$ SR59 $04.02 .03 .03 /$ SR0 6 $04.02 .04 .01 / \mathrm{SR} 27 \quad 04.02 .04 .01 / \mathrm{SR} 2904.02 .04 .02 / \mathrm{SR} 25$ $04.02 .04 .02 / S R 2604.02 .04 .02 / S R 26$

\#S $5.4 .2 / 140 / 1$ :

$04.02 .03 .01 /$ SR5 $904.02 .03 .01 /$ SR60 $04.02 .03 .01 /$ SR61 $04.02 .03 .03 /$ SR0 $804.02 .03 .03 /$ SR0 9

\#S $5.4 .2 / 142 / 1:$

$04.02 .04 .01 / \mathrm{SR} 4904.02 .04 .01 / \mathrm{SR} 50 \quad 04.02 .04 .01 / \mathrm{SR} 52$

\#S $5.12 .1 / 232 / 1$ :

$04.03 .02 .02 /$ SR15 $09.02 .01 /$ SR01 $09.02 .01 /$ SR02 $09.02 .01 /$ SR03

$09.02 .01 /$ SR0 $409.02 .02 /$ SR01 $09.02 .02 /$ SR02 $09.02 .02 /$ SR03

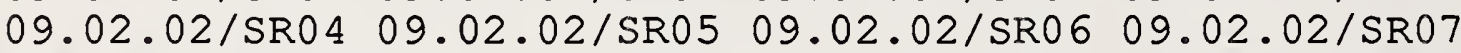

$09.02 .02 /$ SR0 $809.02 .02 /$ SR09 $09.02 .03 /$ SR01 $09.02 .03 /$ SR02

$09.02 .03 /$ SR03 $09.02 .03 /$ SR0 4 09.02.03/SR0 $509.02 .03 /$ SR0 6

$09.02 .03 /$ SR07 $09.02 .03 /$ SR0 $809.02 .05 /$ SR01 $09.02 .05 /$ SR02

$09.02 .05 /$ SR03 09.02.05/SR0 $409.02 .05 /$ SR0 $509.02 .09 /$ SR0 1

$09.02 .10 /$ SR01 $09.02 .10 /$ SR02 $09.02 .10 /$ SRO3 $09.02 .10 /$ SR0 4

$09.02 .10 /$ SR05 09.02.11/SR01 09.02.11/SR02 09.02.11/SR03

\#S $5.12 .1 / 232 / 2$ :

$04.02 .01 .02 / \mathrm{SR} 26$

$04.02 .02 .02 / S R 27$

$04.02 .01 .02 / \mathrm{SR} 27$

$04.02 .02 .02 / S R 26$

$04.02 .03 .02 /$ SR2 8

$04.02 .03 .02 / \mathrm{SR} 2604.02 .03 .02 / \mathrm{SR} 27$

$04.02 .05 .03 /$ SR27 $04.02 .05 .04 /$ SR1 8

$04.02 .05 .03 / S R 26$

\#S $6.1 / 306 / 6$ :

$05.03 /$ SR03 $05.03 /$ SR0 $405.03 /$ SR15

\#S $6.1 / 306 / 8$ :

$04.03 .02 .02 / \mathrm{SR} 2204.03 .02 .02 / \mathrm{SR} 23 \quad 04.03 .02 .02 / \mathrm{SR} 24$

$04.03 .02 .02 / \mathrm{SR} 25 \quad 04.03 .02 .02 / \mathrm{SR} 26$

\#S $6.1 / 306 / 9:$

$04.03 .02 .02 /$ SR0 7 
\#S $6.1 / 307 / 2$ :

$11.01 /$ SR01 $11.01 / \mathrm{SR} 02$

\#S $6.1 / 307 / 3$ :

$11.01 /$ SRO1 $11.01 / \mathrm{SR} 02$

\#S 6.2/308/1:

03.01/SR03 05.01.01/SR02

\#S $6.2 / 308 / 2$ :

$05.01 .01 /$ SR0 $405.01 .01 /$ SR0 5

\#S $6.3 / 309 / 1$ :

$02.03 .03 / S R 2102.03 .03 / S R 2203.01 / S R 0204.01 .03 / S R 08$

$04.02 .01 .01 /$ SR11 $04.02 .01 .01 /$ SR17 $04.02 .01 .01 / S R 23$

$04.02 .01 .02 /$ SR0 $804.02 .02 .01 /$ SR11 $04.02 .02 .01 /$ SR1 8

$04.02 .02 .01 / S R 24 \quad 04.02 .02 .02 /$ SR0 $804.02 .03 .01 / S R 23$

$04.02 .03 .01 / \mathrm{SR} 3204.02 .03 .01 / \mathrm{SR} 3804.02 .03 .01 / \mathrm{SR} 44$

$04.02 .03 .01 /$ SR50 $04.02 .03 .01 /$ SR $6204.02 .03 .01 /$ SR70

$04.02 .03 .01 /$ SR7 $704.02 .03 .01 /$ SR82 $04.02 .03 .02 /$ SR0 8

$04.02 .04 .01 /$ SR1 $604.02 .04 .01 /$ SR24 $04.02 .04 .01 /$ SR30

$04.02 .04 .01 /$ SR35 $04.02 .04 .01 /$ SR $40 \quad 04.02 .04 .01 /$ SR5 3

$04.02 .04 .01 /$ SR73 $04.02 .04 .02 /$ SR0 $604.03 .01 /$ SR10 $05.01 .01 /$ SR 10

$05.01 .01 /$ SR11 $05.01 .02 /$ SR0 7

\#S $6.3 / 310 / 1$ :

$02.02 .02 / S R 1104.02 .04 .01 / S R 6104.02 .04 .01 / S R 68 \quad 04.02 .04 .01 / S R 83$

$04.02 .05 .01 /$ SR1 $604.02 .05 .01 / S R 2404.02 .05 .01 / S R 30$

$04.02 .05 .01 /$ SR3 $804.02 .05 .01 /$ SR $4704.02 .05 .01 /$ SR5 3

$04.02 .05 .01 /$ SR5 $904.02 .05 .01 /$ SR $6404.02 .05 .01 /$ SR8 0

$04.02 .05 .01 /$ SR82 $04.02 .05 .02 /$ SR09 $04.02 .05 .03 /$ SR08 $04.03 .03 /$ SR0 9

$04.03 .04 .01 /$ SR11 $06.01 .02 /$ SR19 $06.01 .02 /$ SR37 $06.01 .02 /$ SR50

$06.01 .02 /$ SR5 4

\#S $6.3 / 311 / 4:$

$06.01 .02 /$ SR36

\#S $6.4 / 313 / 1$ :

$04.03 .04 .01 /$ SR11

\#S $6.5 / 314 / 1$ :

$02.01 .01 /$ SR0 8 $02.01 .01 /$ SR09 $02.02 .03 /$ SR0 $503.01 / S R 04 \quad 03.02 / S R 01$

$03.02 /$ SR02 $05.01 .01 /$ SR01

\#S $6.6 / 316 / 1:$

$06.02 .02 /$ SR09 $06.02 .02 /$ SR10 $06.02 .02 /$ SR1 $106.03 /$ SR0 $006.03 /$ SR0 8 $06.03 /$ SR 15

\#S $6.6 / 316 / 4:$

$04.03 .03 /$ SR10 $04.03 .03 /$ SR12 $04.03 .03 /$ SR13 $04.03 .03 /$ SR14

$04.03 .03 /$ SR 15

\#S $6.6 / 317 / 1$ : 
$04.02 .01 .02 /$ SR24 $04.02 .01 .02 /$ SR2 5

$04.02 .02 .02 /$ SR25 $04.02 .03 .02 /$ SR2 4

$04.02 .04 .02 /$ SR0 $904.02 .05 .02 /$ SR2 5

$04.02 .05 .03 /$ SR2 $404.02 .05 .03 /$ SR2 5

$04.02 .05 .04 / S R 1604.02 .05 .04 / S R 17$

$05.02 /$ SR0 $205.02 /$ SR0 $805.02 /$ SR0 9
$04.02 .02 .02 / \mathrm{SR} 24$

$04.02 .03 .02 / \mathrm{SR} 25$

$04.02 .05 .02 /$ SR2 6

$04.02 .05 .03 /$ SR2 9

$04.03 .02 .02 /$ SR20 $05.02 / S R 01$

$05.03 /$ SR 11

\#S $6.6 / 318 / 1$ :

$04.03 .02 .02 /$ SR11 $04.03 .02 .02 /$ SR13 $04.03 .02 .02 / S R 14$

$04.03 .04 .02 /$ SR03 $04.03 .04 .02 /$ SR0 7

\#S $6.7 / 320 / 1$ :

$06.02 .02 /$ SR0 $506.03 /$ SR0 4

\#S $6.7 / 321 / 1$ :

$04.02 .01 .02 /$ SR21 $04.02 .01 .02 / S R 22$

$04.02 .01 .02 /$ SR2 $404.02 .01 .02 /$ SR2 5

$04.02 .01 .02 /$ SR29

$04.02 .01 .02 / \mathrm{SR} 30$

$04.02 .01 .02 / S R 41$

$04.02 .02 .02 / \mathrm{SR} 23$

$04.02 .02 .02 / \mathrm{SR} 28$

$04.02 .02 .02 / \mathrm{SR} 21$

$04.02 .02 .02 / S R 24$

$04.02 .02 .02 / S R 29$

$04.02 .01 .02 / \mathrm{SR} 23$

$04.02 .01 .02 /$ SR2 8

$04.02 .01 .02 /$ SR 40

$04.02 .02 .02 /$ SR2 2

$04.02 .02 .02 / \mathrm{SR} 25$

$04.02 .02 .02 / \mathrm{SR} 40$

$04.02 .02 .02 / S R 41$

$04.02 .02 .02 / \mathrm{SR} 30$

\#S $6.7 / 322 / 1$ :

$04.02 .03 .01 /$ SR5 $404.02 .03 .01 /$ SR55

$04.02 .03 .02 / S R 21$

$04.02 .03 .02 / \mathrm{SR} 22$

$04.02 .03 .01 /$ SR 57

$04.02 .03 .02 / \mathrm{SR} 24$

$04.02 .03 .02 / \mathrm{SR} 25$

$04.02 .03 .02 / \mathrm{SR} 23$

$04.02 .03 .02 /$ SR 52

$04.02 .03 .02 /$ SR53

$04.02 .03 .02 /$ SR2 9

$04.02 .04 .01 /$ SR 44

$04.02 .04 .01 / S R 45$

$04.02 .03 .02 /$ SR55

$04.02 .04 .02 /$ SR0 9

$04.02 .05 .01 / S R 22$

$04.02 .04 .01 /$ SR 47

$04.02 .05 .02 /$ SR22

$04.02 .05 .02 / S R 23$

$04.02 .05 .01 / S R 23$

$04.02 .05 .02 / S R 25$

$04.02 .05 .02 /$ SR2 6

$04.02 .05 .02 / S R 24$

$04.02 .05 .02 /$ SR2 9

$04.02 .05 .02 /$ SR30

$04.02 .05 .02 / \operatorname{SR} 28$

$04.02 .05 .02 / S R 42$

$04.02 .05 .02 / S R 44$

$04.02 .05 .02 / S R 31$

$04.02 .05 .03 /$ SR 32

$04.02 .05 .03 / S R 33$

$04.02 .05 .03 / S R 21$

$04.02 .05 .03 / S R 44$

\#S $6.7 / 323 / 1$ :

$02.02 .02 /$ SR11 $04.01 .08 /$ SR0 $404.01 .08 /$ SR05 $04.01 .08 /$ SR0 9

$04.01 .08 /$ SR10 $04.02 .05 .03 /$ SR22 $04.02 .05 .03 /$ SR23 $04.02 .05 .03 / S R 24$

$04.02 .05 .03 / S R 25 \quad 04.02 .05 .03 / S R 28 \quad 04.02 .05 .03 / S R 29$

$04.02 .05 .03 /$ SR4 $304.02 .05 .04 /$ SR14 $04.02 .05 .04 / S R 15$

$04.02 .05 .04 / S R 1604.02 .05 .04 / S R 1704.03 .02 .02 / S R 02$

$04.03 .02 .02 /$ SR03 $04.03 .02 .02 /$ SR0 4 04.03.02.02/SR0 7

$04.03 .02 .02 /$ SR10 $04.03 .02 .02 /$ SR12 $04.03 .02 .02 /$ SR13

$04.03 .02 .02 /$ SR14 $05.03 /$ SR1 $705.03 /$ SR1 $805.03 /$ SR1 9

\#S $6.7 / 324 / 1$ :

$04.02 .01 .02 /$ SR1 $804.02 .02 .02 /$ SR18 $04.02 .03 .02 /$ SR1 8

$04.02 .05 .02 /$ SR19 $04.02 .05 .03 /$ SR18 $04.02 .05 .04 /$ SR0 9

$04.02 .05 .04 /$ SR10 $04.03 .02 .01 /$ SR0 $806.02 .02 /$ SRI3 


\section{APPENDIX L}

\section{MODULE CROSS-REEERENCE}

\#X $01:$

$04.01 .03 / \mathrm{SR} 07$

\#X $02.01 .03 .01:$

$02.01 .01 /$ SR0 5

\#X $02.01 .03 .02:$

$02.01 .01 / \mathrm{SR} 06$

\#X $02.01 .03 .03:$

$02.01 .01 /$ SR07

\#X $02.02 .01:$

$02.01 .01 / \mathrm{SR} 01$

\#X $02.02 .03:$

$02.01 .02 .01 /$ SR0 $62.01 .02 .01 /$ SR0 $02.01 .02 .02 / S R 11$

$02.01 .02 .02 /$ SR 12

\#X $02.02 .04:$

$02.01 .01 / \mathrm{SR} 02$

$\# \mathrm{X} 04.01 .03:$

$04.02 .03 .01 /$ SR01 $04.02 .03 .01 / S R 02$

\#X $04.02 .01 .01:$

$04.02 .05 .01 /$ SR4 $404.02 .05 .01 / S R 45 \quad 04.02 .05 .01 / S R 46$

\#X $04.02 .03 .01:$

$04.01 .03 /$ SR09 $04.01 .03 /$ SR10 $04.01 .03 /$ SR 11

\#X $04.02 .03 .03:$

$02.03 .03 /$ SR15

\#X $04.02 .04 .01:$

$04.01 .04 /$ SR0 $704.01 .04 /$ SR0 8

\#X 04.02 .05 .01 : 


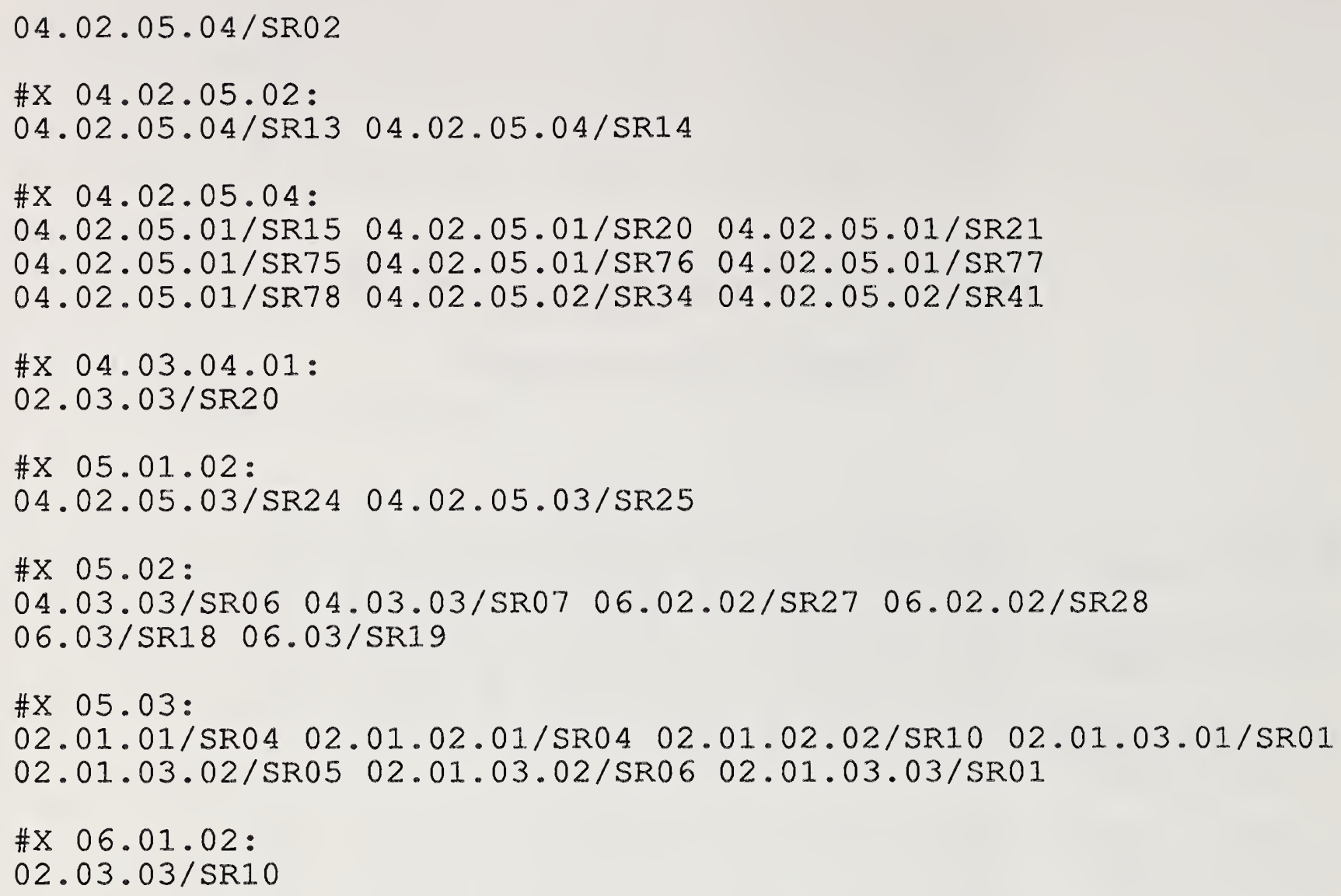


